\title{
AN ENVIRONMENT TO SUPPORT PROBLEM SOLVING
}

\author{
PIETER W.G. BOTS
}

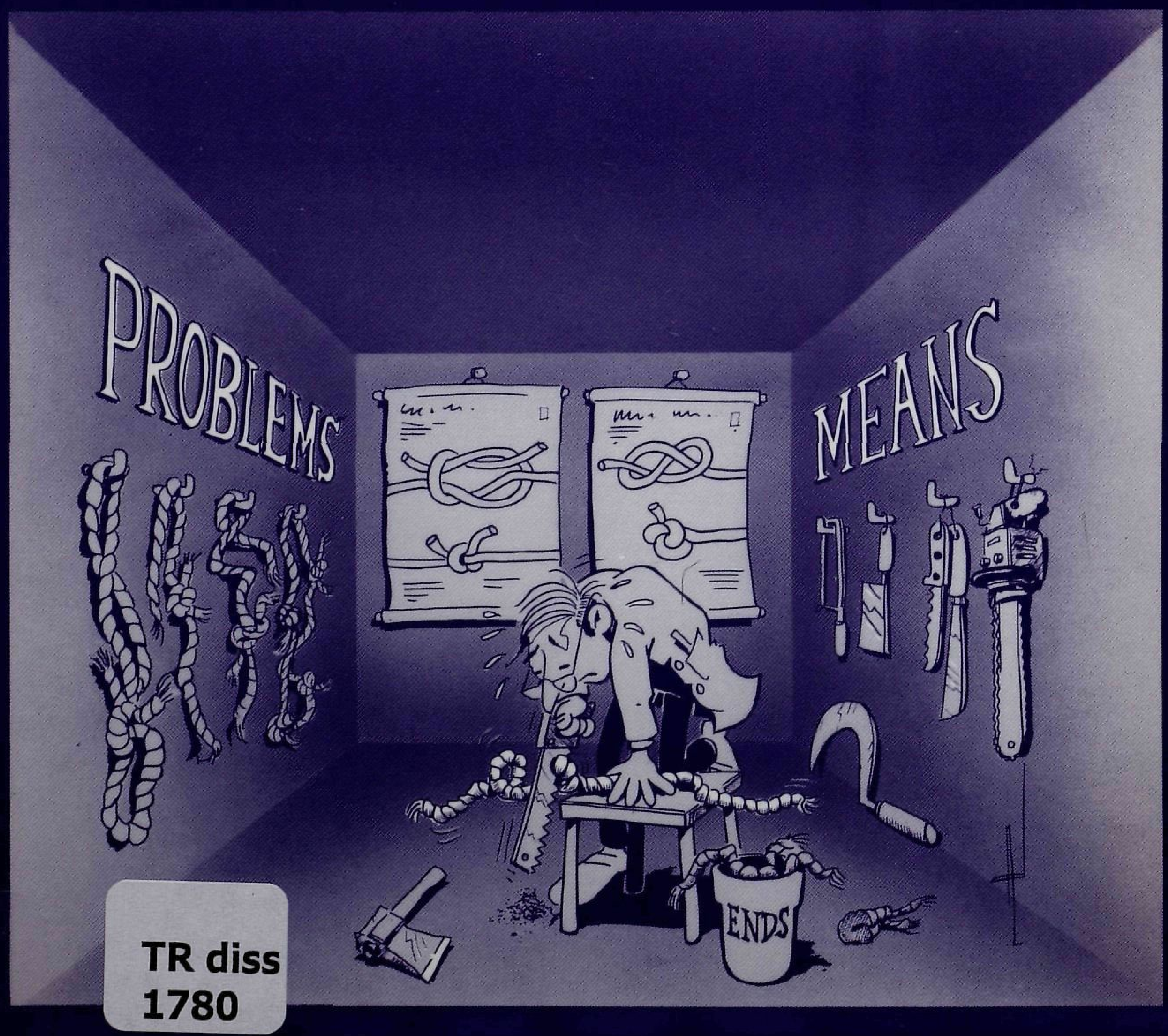


AN ENVIRONMENT

TO SUPPORT PROBLEM SOLVING 
AN ENVIRONMENT

TO SUPPORT PROBLEM SOLVING

Proefschrift

ter verkrijging van de graad van doctor aan de Technische Universiteit Delft

op gezag van de Rector Magnificus, Prof. drs. P.A. Schenck

in het openbaar te verdedigen ten overstaan van een commissie aangewezen door het College van Dekanen op maandag 18 december 1989 te 14.00 uur

door

Petrus Wilhelmus Gerardus Bots geboren te Oegstgeest

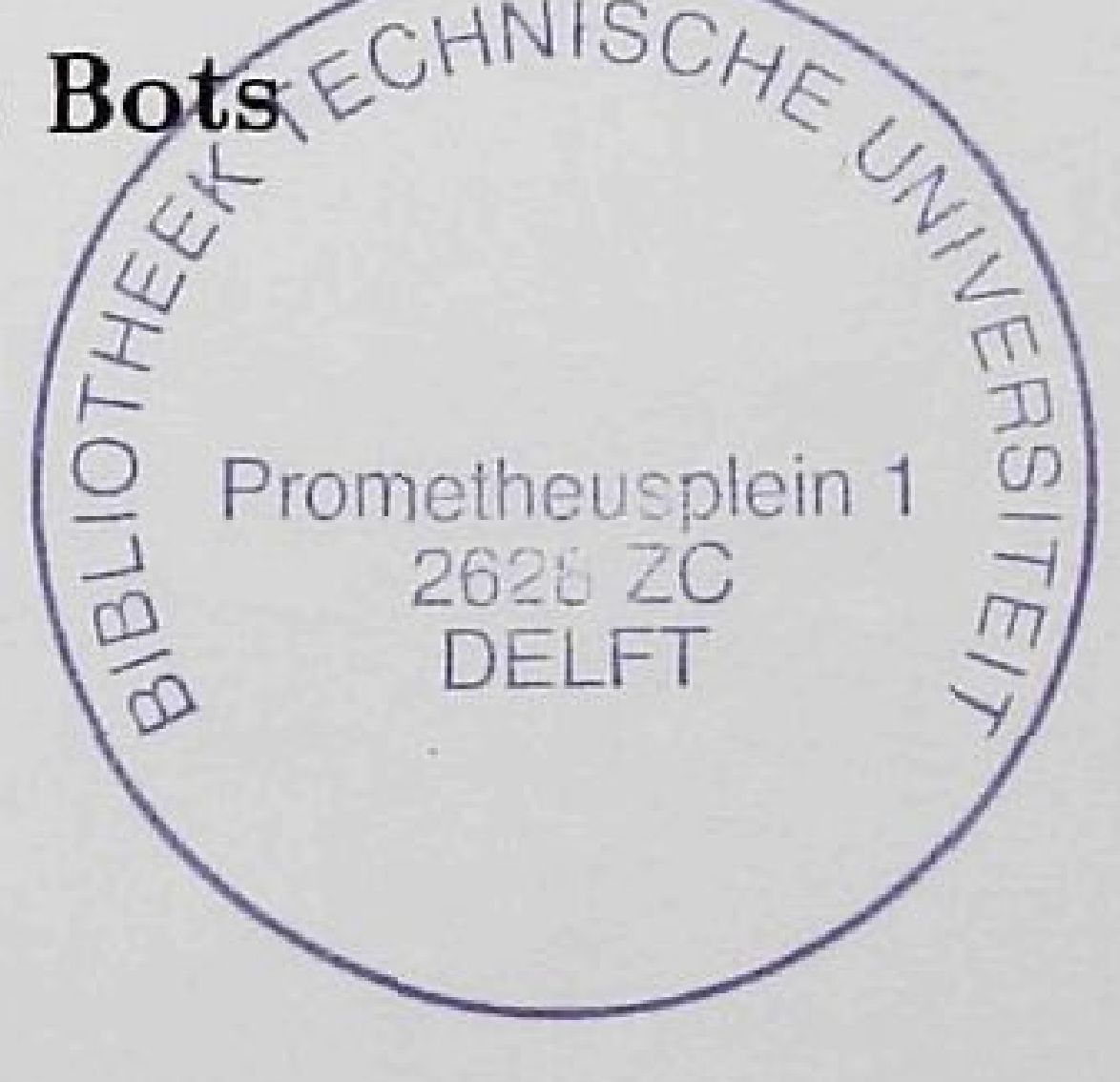


Dit proefschrift is goedgekeurd door de promotoren:

Prof. Dr. H.G. Sol en Prof. Dr. A.A. Verrijn Stuart

en door de leden van de promotiecommissie:

Prof. Drs. B.K. Brussaard

Prof. Dr. Ir. J.J.M. Evers

Prof. Dr. K.M. van Hee

Prof. Dr. H. Koppelaar

Prof. Dr. D.J. McConalogue

\section{STELLINGEN}

behorende bij het proefschrift

An Environment to Support Problem Solving

Pieter W.G. Bots

18 december 1989 
en

Pro

Pro

Pro

Pro

Pro
De "Data Envelopment Analysis" techniek voor het meten van de efficiëntie van beslissingseenheden in organisaties kan worden verbeterd door haar te combineren met de in dit proefschrift beschreven techniek voor taakanalyse.

A. Charnes, C.T. Clarke and E. Rhodes, "Measuring Efficiency of Decision Making Units," European Journal of Operational Research, Vol. 2 (1978), pp. 429-449.

A.Y. Lewin and J.W. Minton, "Determining Organizational Effectiveness: Another Look, and an Agenda for Research," Management Science, Vol. 32, No. 5 (mei 1986), pp. 514-538.

Ook indien voor ondernemingen met een volcontinudienst het vijfploegensysteem en de daarbij behorende werkweek van 33.6 uur de norm wordt zullen roosterplanners sterk gebaat blijven bij de in Sectie 5.4 van dit proefschrift beschreven computerondersteuning.

Taakanalyse zoals toegepast in Hoofdstuk 7 van dit proefschrift doet afbreuk aan het leereffect dat als regel met het spelen van een management game wordt beoogd.

Overlappende vensters in een "windowing" applicatie zijn onwenselijk; zij zijn onnodig wanneer de taak die door deze applicatie wordt ondersteund naar behoren zou zijn geanalyseerd.

ndien binnen een organisatie geen bindende richtlijnen worden gesteld ten aanzien van het gebruik van electronisch agendabeheer zal dit gebruik ook op lange termijn verwaarloosbaar zijn. P.W.G. Bots, M.A.H. Cohen en H.G. Sol, Evaluatie Stimuleringsplan Automatisering, Rappor

Bij het ontwikkelen van informatiesystemen wordt nog steeds onvoldoende rekening gehouden met het feit dat niet alleen de gebruikers maar ook de ontwerpers cognitieve beperkingen hebben. Voor effectief onderzoek naar informatiesystemen zou beperkte rationaliteit het uitgangspunt moeten zijn.

\section{VII}

Het feit dat Borland haar Turbo Pascal uitbreidt met faciliteiten voor object management nog vóordat het gebruik van dynamische arrays wordt ondersteund is tekenend voor de huidige commerciële waarde van het label "object-georiënteerd".

\section{VIII}

Het succes van het computerprogramma Eurisko zoals beschreven door Lenat berust op het feit dat de daarin gebruikte taal voor het beschrijven van getaltheoretische fenomenen door een formele grammatica kan worden gegenereerd. De verwachting dat een computer vergelijkbare "exploratieve intelligentie" kan vertonen in domeinen die niet met een formele taal kunnen worden beschreven is ongegrond. Lenat, D. "Computer Software for Intelligent Systems", Scientific American, Vol. 251, No. 3
(september 1984), pp. 204-211.

\section{IX}

Lijders aan lichte tot min of meer ernstige vormen van dyslexie zijn voor het aanleren van een taal als regel zeer geschaad door het gebruik van zogeheten "natuurmethoden"; daarentegen zijn zij meer gebaat bij kennis van duidelijke grammaticale regels.

$$
\mathrm{X}
$$

Het is opmerkelijk hoe een typisch Westerse vinding als een gelduitgifteautomaat een on-Westers element als in lange rijen buiten wachtende mensen aan het Nederlandse straatbeeld heeft toegevoegd.

$$
\text { XI }
$$

Indien als gevolg van een theoretische of technologische doorbraak kan worden aangetoond dat $P=N P$ zal dit ingrijpende consequenties hebben voor met name de verkeerssituatie in grote steden.

XII

Science fiction is opium voor wetenschappers. 
Dit proefschrift is goedgekeurd door de promotoren:

Prof. Dr. H.G. Sol en Prof. Dr. A.A. Verrijn Stuart

en door de leden van de promotiecommissie:

Prof. Drs. B.K. Brussaard

Prof. Dr. Ir. J.J.M. Evers

Prof. Dr. K.M. van Hee

Prof. Dr. H. Koppelaar

Prof. Dr. D.J. McConalogue 


\section{Bots, Petrus Wilhelmus Gerardus}

An Environment to Support Problem Solving /

Petrus Wilhelmus Gerardus Bots. - [S.I. : s.n.]. - III.

Met diskette

Proefschrift Delft. - Met lit. opg. -

Met samenvatting in het Nederlands.

ISBN 90-9003151-0

SISO 366.2 UDC 681.3:658.012.4(043.3)

Trefw.: informatiesystemen ; organisaties

probleemoplossen ; organisaties.
PREFACE

1 INFORMATION TECHNOLOGY AND INFORMATION SYSTEMS

1.1 Introduction $\ldots \ldots \ldots \ldots \ldots \ldots \ldots \ldots$

1.2 Decision support systems $\ldots \ldots \ldots \ldots \ldots$

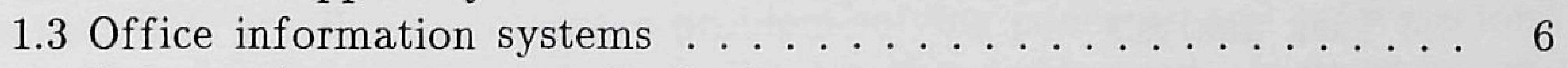

1.4 Information systems: technologies converge . . . . . . . . . . 9

1.5 Research question and outline of the study ............ 11

2 THEORIES ON PROBLEM SOLVING

2.1 Introduction .................... 13

2.2 The "structuredness" of problems $\ldots \ldots \ldots 14$

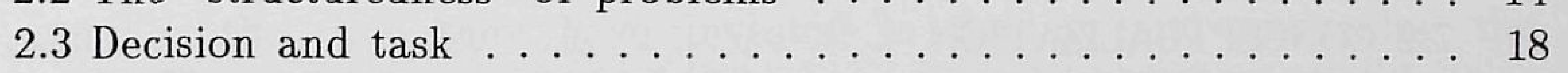

2.4 The coordination problem $\ldots \ldots \cdots \cdots \cdots \cdots \cdots \cdots \cdots \cdots \cdots$

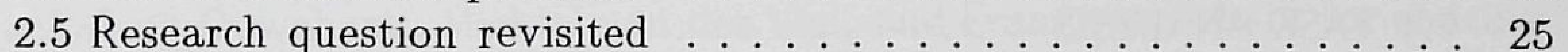

3 SUPPORTING PROBLEM SOLVING

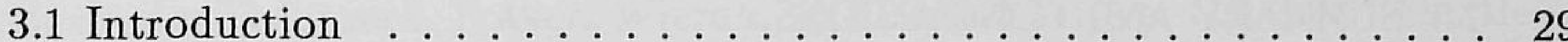

3.2 Problem conceptualization $\ldots \ldots \ldots \ldots \ldots \ldots \ldots$

3.3 Problem specification $\ldots \ldots \ldots \ldots \ldots \ldots$

\section{A PROBLEM SOLVING SUPPORT ENVIRONMENT}

4.1 Introduction . . . . . . . . . . . . . . . . 51

4.2 Analysis of the problem solving task . . . . . . . . . 52

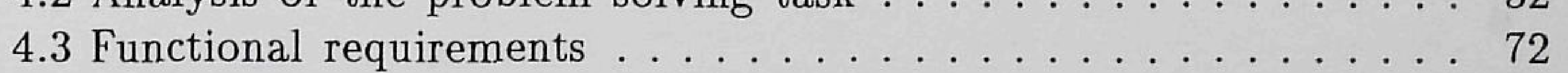

4.4 Implementation issues $\ldots \ldots \ldots \ldots . \ldots \ldots$

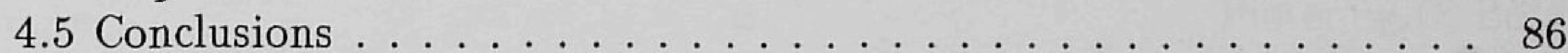


5 INDIVIDUAL PROBLEM SOLVING SUPPORT

THE CASE OF SHIFTWORK PLANNING

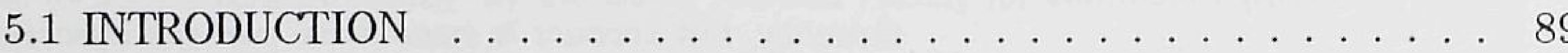

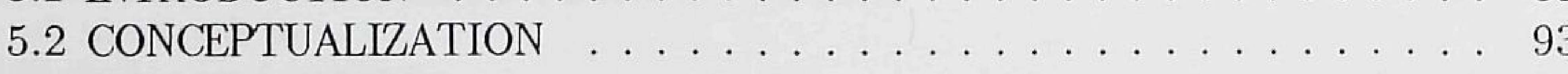

5.3 SPECIFICATION . . . . . . . . . . . . . . . . . . . . 99

5.4 TASK ANALYSIS $\ldots \ldots \ldots \ldots$

5.5 DECISION SUPPORT . . . . . . . . . . . . . . . . . . 112

5.6 SUMMARY AND CONCLUSIONS $\ldots \ldots \ldots \ldots \ldots \ldots \ldots$

\section{GROUP PROBLEM SOLVING SUPPORT}

THE CASE OF INTERNATIONAL TRANSFER PRICING

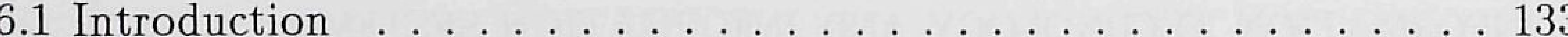

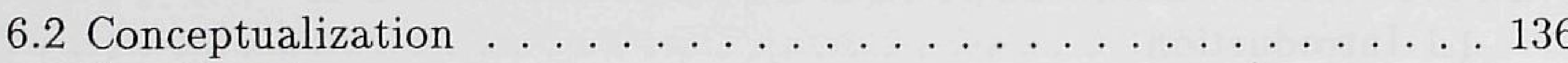

6.3 Specification . . . . . . . . . . . . . . . . . . . . 144

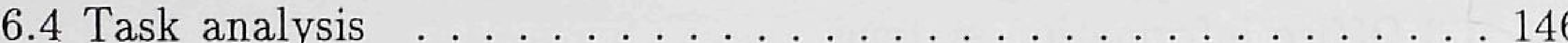

6.5 Decision support . . . . . . . . . . . . . . . . . 164

6.6 Summary and conclusions . . . . . . . . . . . . . . . 169

\section{ORGANIZATIONAL PROBLEM SOLVING SUPPORT}

THE CASE OF A MANAGEMENT GAME

7.1 INTRODUCTION .......................... 171

7.2 CONCEPTUALIZATION . . . . . . . . . . 174

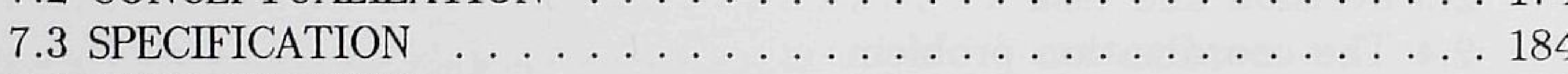

7.4 TASK ANALYSIS $\ldots \ldots \ldots \ldots \ldots \ldots \ldots \ldots \ldots \ldots \ldots$

7.5 DECISION SUPPORT $\ldots \ldots \ldots \ldots \ldots$

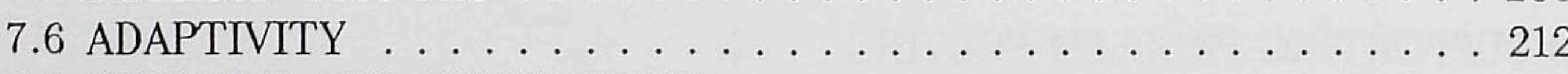

7.7 SUMMARY AND CONCLUSIONS $\ldots \ldots \ldots \ldots \ldots \ldots \ldots \ldots$

\section{EPILOGUE}

8.1 INTRODUCTION . . . . . . . . . . . . . . . . . 217

8.2 A REVIEW OF HYPOTHESES . . . . . . . . . . . . . . . 217

8.3 CONCLUDING REMARKS . . . . . . . . . . . . . . . 220

REFERENCES

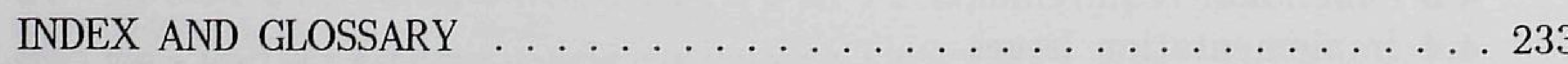

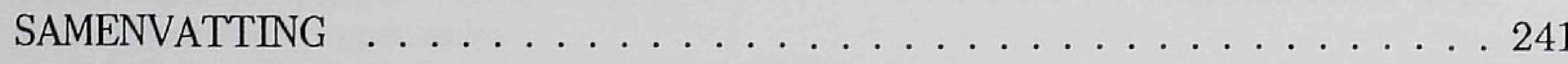

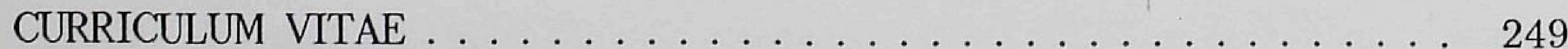

\section{PREFACE}

The actors are at hand; and, by their show, You shall know all that you are like to know. A MIDSUMMER-NIGHT'S DREAM (Act. V Sc. I)

Perceiving problems and subsequently trying to solve them is typical for human beings. This thesis addresses the issue of supporting decision makers in their problem-solving tasks by means of information technology. It is assumed that these decision makers have cognitive limitations, and that the main objective of developing information systems should be to reduce these limitations. This study shows that it is possible to integrate problem-solving concepts and information technology in an environment that facilitates the design and construction of information systems to support decision processes.

Many people have contributed in various ways to the writing of this thesis. Acknowledgements are due, first, to Henk Sol and Alex Verrijn-Stuart for the opportunities they have given me, for the many lengthy discussions we have had, and for the time they have invested in reading and re-reading the manuscript. Their constructive criticism has been invaluable. Secondly, I want to thank Jaap Ouwehand, Michiel van der Ven, and Frans van Schaik for sharing with me their expertise in the areas of shiftwork planning, international transfer pricing, and gaming, respectively. I owe my colleagues, in particular Remko Dur, Alexander Verbraeck, Folkert Wierda, and Gerard Wijers, for their relentless challenging of both my wit and my working hours. My thanks also go to Dennis McConalogue for kindly showing me "the elements of style". I am grateful to my parents and friends for their encouragement and support. Most of all, want to thank Hermieneke for her patience and her great sense of humor, especially when pointing out in a million ways that there is more to life than writing a dissertation. 


\section{INFORMATION TECHNOLOGY}

AND INFORMATION SYSTEMS

Once upon a time, computers were very simple. Those days are long gone. - Andrew S. Tanenbaum

\subsection{INTRODUCTION}

Information systems as a field of research is characterized by a strong technology push. Until recently, technology was the bottleneck to progress in this field, and new ideas had to wait for new developments in hardware and software before they could be realized. Ten years ago, Verrijn-Stuart (1979, page 157) pointed out this peculiar relationship between technology and theory building. A striking example is the history of "Hypertext", reported by Smith and Weiss (1988). Hypertext applications have only recently become popular, even though the phrase was coined by Nelson as early as 1967. The Hypertext concept can be traced back even further (Bush 1945). At that time, however, implementation was not feasible.

Today technology is no longer the constraining factor in information systems research. Optical data communication and storage devices, high performance RISC-processors, and non-Von Neumann architectures challenge, rather than inhibit, human imagination.

Man cannot refuse this challenge, and as a consequence he changes the world he lives in. Progress in information technology coincides with the current transition from an industrial to a post-industrial society. This new society is characterized by more and increasing knowledge, complexity, and turbulence. As such, it imposes greater demands on organizations. Specifically, it requires more frequent and faster organizational decision making, and it causes a need for a more continuous and wider-ranging organizational information acquisition (Huber 1984a, page 933). Clearly, this affects the shape of information systems.

The present transition to an information society is reflected in the multitude of views that can be found in literature. Information systems is a young research area, and its terminology is often confusing. What is the exact meaning of management information systems, decision support systems, executive information systems, expert systems, or office information systems? The lack of a sound conceptual foundation makes it hard to tell the difference between buzzwords and concepts that really matter. However, we do not believe that the problem of correctly labeling the wide variety of information technologies is a fundamental issue. One should look for "megatrends" (Naisbitt 1982): trends that go beyond phrases currently in vogue, trends that will determine the future developments in information systems. 
A first megatrend is that information technology can and will be used to gain a sustainable competitive advantage. Studies conducted at the Harvard Business School (McFarlan et al. 1982; Parsons 1984) highlight this strategic potential of information technology, while Keen (1986) demonstrates how telecommunications can be used for opening new product/market combinations, or for changing an organization's competitive position. Although barely exploited, the benefits of inter-organizational information systems are becoming more and more evident. This growing awareness of information technology as a competitive weapon is not a passing trend. It will greatly affect the way organizations develop and use information systems.

A second megatrend is that organizational decision making is, or soon will be, the primary organizational activity. Simon formulated this belief as early as 1960. Later he stated that decision making in the post-industrial society will be a great deal more complex than the decision making of the past, and that as a result the decision-making process, rather than the processes contributing immediately and directly to the production of an organization's final output, will bulk larger and larger as the central activity in which that organization is engaged (Simon 1973a, page 269). Bosman and Sol (1985, page 81) give decision making a central place in information systems design. Huber and McDaniel (1986a, b) go even further along this line when they conclude that organizations should be designed primarily to facilitate the making of organizational decisions. We strongly believe that this "decision focus" will determine the shape of organizational information systems.

Finally, a third megatrend is that information technology will be aimed at improving human performance. Human judgement itself cannot be automated. Therefore, rather than being replaced, people in the information society should be provided with "power tools" (Brown 1983) or even "white collar robots" (Tsichritzis et al. 1987) to extend their capabilities. This, too, will have a lasting influence on the design of information systems.

Using a slightly different terminology, Sprague (1986, page 19-20) actually puts all three convictions together when he suggests that the ultimate mission of information systems in an organization is "to improve the performance of information workers in organizations through the application of information technology". Although he admits that this mission is not very specific, with ambiguous words such as "improve" and "performance", he points out two important implications. First, it establishes people - information workers - as the target of information systems. Second, it means that information systems should increase performance. Merely producing reports, or supporting activities, or even "getting the right information to the right person at the right time" falls short unless the result is performance improvement.
In the remainder of this chapter we shall investigate how these ideas have evolved. In Section 1.2 and 1.3 we trace the history of decision support systems and office information systems, and analyze their characteristics. In Section 1.4 we present our view on what an information system is from an organizational perspective, and point out what consequences this has for information systems design. Finally, in Section 1.5 we formulate a tentative research question, and give an outline of the study reported in this thesis.

\subsection{DECISION SUPPORT SYSTEMS}

The technological developments of the last fifteen years have stimulated the efforts of information systems researchers to apply information technology to problems that were previously thought of as unsuited for computer support. Such problems are often referred to in the literature as "ill-structured problems" (see Section 2.2). Solving ill-structured problems requires a special kind of information technology. Gorry and Scott Morton are generally credited with being the first to describe the concept of decision support systems (DSS). In their article "A Framework for Management Information Systems" (1971) they describe a new class of information systems. These systems distinguish themselves from traditional information systems in terms of both design and use: in addition to the usual data access and reporting capabilities they incorporate analytical capabilities, and they are intended for direct use by decision makers and their staff, rather than data processing professionals or clerks.

Thus, according to Sol (1985, page 3), the short history of DSS begins with a shift in paradigm which resulted in the DSS concept. Once the potential of this new approach to information systems is recognized, the DSS movement starts. As initial successes are reported, this movement gradually gains momentum. In the early 1980's the DSS movement turns into a bandwagon. Software developers suddenly pass off existing products as DSS, and research disciplines outside the field of information systems, recognizing the commercial value of the DSS label, also promote their software and services as decision support.

This "bandwagon effect" was possible because there was no precise, generally accepted definition that limited the scope of DSS. In 1978, Keen and Scott Morton defined a DSS as a system that focusses on managers' decisionmaking activities and needs while extending their abilities. A DSS assists managers in their decision processes in ill-structured tasks, and supports, rather than replaces, managerial judgement. They defined its ultimate purpose as the improvement of the effectiveness of decision making rather than its efficiency. This definition leaves considerable room for interpretation, and in the years following its introduction many researchers sought to refine it, looking for a 
more formal, a more conceptual, or a more technical definition, depending on their particular perspective and purpose.

The resulting variety of interpretations in itself became an object of study. Ginzberg and Stohr (1982), for example, examine a number of definitions in order to elicit the issues most central to developing more effective DSS. Looking at DSS technology, they trace the modelling aspect back to the concept of a "decision calculus" (Little 1970). They contrast Moore and Chang's (1980) definition in terms of usage pattern and system capabilities with the one by Keen (1980) in terms of adaptive design, review the data-oriented versus modeloriented designs (Alter 1977) and the institutional/ad hoc dichotomy (Donovan and Madnick 1977), and compare this with the distinction between DSS generators and specific DSS (Sprague 1980) and the procedural versus nonprocedural classification scheme presented by Bonczek et al. (1981). Inevitably they end up by adding yet another definition to the already extensive collection. Nevertheless, their survey sheds light on the developments in the DSS field. They conclude that DSS definitions should not limit the range of such systems by requiring that specific components be included, or that specific usage or development patterns should be followed. Their final message is that research should not focus on these issues. Instead, it should be directed at understanding decisions and decision making.

Today, 15 years since the term DSS came into use, there still is no generally accepted definition. Although the lack of a sound conceptual foundation has been sharply criticized (Naylor 1982), this situation has not stopped the progress in the field (Sprague and Watson 1986; McLean and Sol 1986; Lee et al. 1988). Nevertheless, Elam et al. (1984) and Sol (1987) plead for a more restrictive definition of DSS. According to Sol only "environments" that support all stages of the problem-solving process truly deserve the DSS label.

Likewise, Keen (1987) explicitly states that the definitional issue has to be solved. But not by attempting once more to produce the definition of DSS. Years of discussion should teach us that there can be no definition of decision support systems, only of decision support. The technology that DSS draws on changes constantly, and there is no independent or idiosyncratic technical base for it. As new technologies become available and suitable, new types of DSS will be built. The goal is decision support, DSS is but a vehicle.

Bosman (1985) arrives at a similar conclusion. From the work of Ginzberg and Stohr (1982), a survey by Bennett (1983), and some other DSS definitions (Thierauf 1982; Belew 1985), he concludes that two different kinds of "attributes" can be distinguished that are used to delineate DSS. First, there are those connected with the process of decision making in organizations. Then there are those that directly relate to information processing. The former are by far the most important. Research should focus on decision making. Sol (1982) demonstrated how simulation models can be used to support the conceptualiz- ation and specification phases of problem solving (see sections 2.4 and 3.2). Building on these ideas, Bosman (1985, page 33) states that it is possible to support any kind of decision process, but that constructing one generic model to support any decision maker is not possible. Different decisions, as well as different phases in a decision process, require different models. Normative models, such as those developed in the fields of management science and operations research, emphasize the selection of the best alternative once a problem and the alternative solutions have been specified. For problem detection and formulation, different models are needed - models that describe decision processes and allow for experimentation with different scenarios. Expert systems, for instance, can be viewed as instances of this class of models (Bosman and Sol 1985).

In our opinion, "expert systems" are a software technique for decision support. Similar to DSS, there is no single definition of expert system (Chandrasekaran 1984, page 42-44). Pragmatics might simply define an expert system as any system that has been developed using an expert system shell. The concept of an expert system shell fits within Sprague's definition of a DSS generator, which puts an expert system in the place of a specific DSS.

Barr and Feigenbaum (1981, page 9) characterize an expert system as a system that consists of a collection of facts and inference rules (the system's knowledge base), and a reasoning mechanism (the inference engine) that can use the knowledge represented by the facts and rules to solve a specific problem and supply the user on request with information on its reasoning. This characterization is clearly in accordance with the DSS definition given by Bonczek et al. (1981) in terms of a language system, a knowledge system, and a problem processing system.

Ben-Bassat (1985, page 673) defines an expert system as a system that has knowledge of a certain application domain, and can be consulted in much the same way as one could consult a human expert. But what is the role of a human expert, other than to support a decision maker by giving advice and by suggesting and evaluating different alternatives?

There is perhaps one argument that supports viewing expert systems not as a technique but as a distinct class of information systems: the idea that expert systems are meant to actually replace human decision makers, while DSS are meant to support them (see page 3 ). But this view is difficult to maintain. Apart from the category of process control systems that incorporate certain expert systems technologies (Gluss 1973; Asai and Tanaka 1975; Stoica and Scarlat 1975) we did not find evidence in the literature of any expert system truly acting in the place of a human decision maker, that is to say, without a human monitoring its reasoning process and checking its suggestions. On the 
other hand, there is evidence that expert system technology can be integrated with DSS (Turban and Watkins 1986; Van Weelderen and Sol 1989).

Summarizing this section, we can say that the number and diversity of information technology applications is increasing rapidly, and the harder we try to strictly categorize these applications, the clearer it becomes that this effort is futile. Decision support systems, executive information systems, expert systems, they all are in fact information systems to support decision processes, a concept put forward by Sol (1989).

Research in this particular area of information systems has brought us a number of useful concepts. It has directed our way of thinking towards illstructured problem solving. It has changed our modeling method, extending the set of models of solutions to include models of problem situations and decision processes. It has led to new working methods, such as prototyping and adaptive design, but at the same time it created a need for new methods of control: middle-out design (Ness 1975) and value analysis (Keen 1981). Most important, DSS research gives decision making a central place in information systems design.

\subsection{OFFICE INFORMATION SYSTEMS}

Office information systems (OIS) emerges as a specific field of research in the late 1970's (Zloof and De Jong 1977; Ellis and Nutt 1980). This diversion from the mainstream in information systems research is for the greater part due to advances in information technology. Time-sharing systems suddenly offer possibilities for the automation of office work that batch-processing simply could not provide. At first research focusses mainly on these new technical capabilities, resulting in office tools such as full-screen editors, word processors, electronic mail, and calendar systems. But as these tools start to affect the nature of office work, the need for conceptual office models is felt

Over time, various models and methodologies for conceptual OIS design have been developed. As part of a comparative review, Bracchi and Pernici (1984) distinguish between three conceptual views that lead to different approaches in the analysis of office work: a technical view, aimed at measuring the physical dimension of office work in terms of throughputs and execution times, an organizational view, perceiving an office's global organizational structure and goals, and finally a socio-technical view, considering an office in terms of the tasks to be performed by each organizational unit, and the goals, decision rules and resources involved in the execution of these tasks. All three views are essentially analytical.
Hirschheim (1986) emphasizes that in addition to this analytical perspective, there is the "interpretivist" perspective. The analytical perspective sees an office as an environment in which people perform a variety of functions necessary to keep the organization in operation. These functions are viewed mainly as formal and structured actions or activities. The interpretivist perspective considers the office in terms of mostly ill-structured and informal human action. From this perspective, office work is viewed in quite different ways, for example in terms of actors and roles (Mintzberg 1973), decision makers and cognitive styles, or social actions mediated through language (Flores et al. 1988).

Different views lead to different office models. Bracchi and Pernici classify these in four main categories: data-based models, process-based models, agentbased models, and mixed models. Generally, data-based models group data into structures similar to paper forms in the traditional office (Tsichritzis 1979, 1982; Zloof 1981; Hämmäinen et al. 1986). Office activities are modeled as series of Znescess-based models describe offices in terms of activities performed (concurrently) by office workers and/or computers. Process-based models most often find their origin in condition/event nets or Petri nets (Zisman 1978. Croft and Lefkowitz 1984). Agent-based models differ from the previous two classes in the sense that their goal is to represent and analyze office workers' roles. Data and activities are considered only in relation to their manipulators or executors. Thus, agent-based models make it easier to describe structural changes in the office (Aiello et al. 1984). Finally, mixed models explicitly assume more than one type of element as the basis for system specification. Bracchi and Pernici point out that most of the recent of fice models belong to this mixed category (Ellis and Bernal 1982; Gibbs 1982). Note that this means recent in 1984. Nevertheless, their remark remains valid (Kappel et al. 1986; Pernici and Verrijn-Stuart 1989).

Although there is a variety of views and modeling approaches, the question of what is an office information system has received much less attention than the definitional problem in the DSS area. Criticism has been aimed at the importance of the field, rather than at the absence of a conceptual foundation (Poppel 1982). Most likely this is due to the fact that there has never been an office systems "bandwagon" that caused a need for a consensual definition to limit entry and interpretation.

The field of office information systems is still developing. In the past five years we have witnessed a shift of focus towards the problem-solving aspect of office work. From experiments with the OMEGA knowledge system Barber (1983) concludes that office work is a problem-solving activity that can be supported with the use of knowledge-embedding languages. Lochovsky (1983) sures this view and presents a slightly different knowledge-based approach to OIS development. Hewitt (1986) demonstrates that offices can be viewed as "open systems", but his open system paradigm so far has not led to any 
practical results. More tangible is the "Procedure Automation and Problem Solving" (PAPS) system described by Woo and Lochovsky (1986a, b). This specific office information system is based on the belief that the procedural view of office work can and should be combined with the problem-solving approach. Earlier, Panko and Sprague (1984) made the distinction between Type I and Type II office work, which in turn corresponds to the even older distinction between structured and ill-structured problems (see Section 2.2). Type I activities are characterized by a large volume of relatively low-cost transactions, well-defined procedures, structured data, and an emphasis on efficiency. Type II work consists of fewer but more costly transactions for which no definite procedure exists, transactions that are performed using less structured and often ambiguous data, with an emphasis on effectiveness. Panko (1984) applied this Type I/Type II dichotomy to a number of individual offices. The distinction proved useful, but too restricted to cover all kinds of office work. Woo and Lochovsky circumvent this problem by allowing tasks to be decomposed into a hierarchy of subtasks where only the leaf-node subtasks are either Type I or Type II office work. Based on this view, the PAPS system supports integration of support for Type I and Type II office work.

Another development, independent from the shift towards a problem-solving view on office work, is the growing popularity of object-oriented modeling. Since the use of object-oriented modeling in information systems design can be traced back to the mid-1970's (Holbæk-Hanssen et al. 1975; Sol 1977, 1982), this development should not be seen as a recent theoretical break-through, but as a clear manifestation of advancing information technology.

Concepts related to object-oriented programming languages (Dahl et al. 1970; Goldberg and Robson 1983; Meyer 1988), such as information hiding, encapsulation and inheritance mechanisms, have become fundamental to office modeling approaches. Although these concepts remain essentially the same, regardless of the specific approach in which they are used, this "objectorientation" has not significantly reduced the diversity of office models (VerrijnStuart and Bots 1987).

Summarizing this section, we can state that information technology today facilitates a wider range of choices in organization and control of office work than was previously possible. Research in office information systems has progressed from the design and construction of office tools to the development of computer-based office environments that provide means to describe and support office tasks. Similar to DSS, research in OIS has affected our view of what information systems are and how they should be designed. It has stimulated both object-oriented and task-oriented thinking.

\subsection{INFORMATION SYSTEMS: TECHNOLOGIES CONVERGE}

Looking at the developments in decision support systems and office information systems, we see striking similarities in the way these areas have gained recognition and respect, and in their use of new technologies. Office information systems have grown beyond mere office tools, and are now moving slowly towards integrated environments to support office work. The DSS field saw a similar development: from specific DSS to DSS generator. But there are more fundamental correspondences.

Office systems design methods such as the one proposed by Woo and Lochovsky (1986a, b) can easily be mapped onto Sprague's framework for DSS development, with its classic modelbase/database/dialogue system partition. It is therefore not surprising that decision support and office technologies are used in combination. A typical example of such a "hybrid technology" information system is the group decision support system described by Jarke (1986). This MEDIATOR system successfully combines OIS communication technologies with DSS tools, and serves as the major communications channel for knowledge sharing, opinion exchange, and negotiation.

Some ten years ago, Keen (1980) advocated a task-oriented approach to DSS development. Today, both the DSS and the OIS area show a task-oriented way of thinking. Ellis and Naffah (1987) even go as far as to define an office information system as "a system which assists office workers in a variety of tasks". With respect to the working method, the main difference between DSS and OIS development has perhaps been that the latter were mostly designed using a bottom-up approach, rather than the middle-out approach that characterizes DSS. But here too, we see a convergence. As office information systems grow beyond toolboxes and start to focus on problem solving, it is only natural that DSS-like techniques are adopted.

Both DSS and OIS practitioners believe that information technology can significantly improve organizational performance and effectiveness if it is utilized as an opportunity to re-design the content of jobs. New information technology permits relaxing constraints on what tasks workers perform, the location where these tasks are performed, and the control systems to ensure that they are performed (Olson and Turner 1985, page 264). As a result, information systems will take the shape of networks of workplaces where the tasks - both regular and ad hoc - of information workers are supported (Sol 1988).

Still, the most important aspect of this convergence of technologies and ideas is the growing awareness that when developing information systems we cannot keep structured and ill-structured problems strictly separated. According to Sprague (1986, page 21 and 24), real improvement of the performance of information workers in organizations requires a focus on Type II task support. But Sol (1988) argues that both types of work are performed on the same workplace, and that the focus therefore should be on the integration of Type I 
and Type II task support. The developments in the field of office information systems seem to uphold this view.

Improving the performance of an organization can be considered from three perspectives (Bots and Sol 1988; Sol 1988):

1. From the micro-perspective we look at the task improvement of people in their workplace. It is at this level that we see the third megatrend we identified earlier: the focus on human performance.

2. From the meso-perspective we are concerned with the coordination of workplaces in an organizational setting, with the aim of improving the performance of the organization as a whole. The shift of attention towards organizational decision making (the second megatrend) has its greatest impact at this level.

3. Looking from the macro-perspective we extend our scope to include several organizations, viewing these organizations as single entities, again with the aim of improving the overall performance. At this level we should consider the use of information technology as a competitive weapon (the first megatrend).

These three perspectives on the improvement of organizational performance can be related directly to information systems when we adopt the information paradigm described by Brussaard and Tas (1980, page 822). They view organizations as dynamic systems. Any such system can be modeled in terms of an "information system" (IS) and a "real system" (RS), where the former determines the behavior of the latter. Control is realized through communication (information exchange). The RS sends messages concerning its state to the IS, which interprets these messages. The IS then acts according to the obtained information by sending messages that will affect the RS, causing its state to change.

The IS and RS components themselves can be described in terms of IS/RScombinations at various levels of abstraction. This concept is called the "recursion principle" of the information paradigm. It can be applied inward until an appropriate level of detail is reached, or outward, up to an appropriate level of abstraction.

At any level of abstraction an IS/RS-combination has an environment that comprises everything the IS does not control. The environment may send messages to the IS, thus possibly affecting the control it exerts over the RS, but it may not send messages directly to the RS, for this would imply that the IS does not totally control the RS. We shall refer to this restriction as the "demarcation rule".

When describing an organization from the micro-perspective, every task that is performed within that organization is viewed as an IS/RS-combination.
The level of abstraction is low, the focus lies on the organization's primary processes and the possible improvement thereof. Relationships between tasks are not made explicit, as they are considered to be part of the IS/RS-combinations' environment.

When looked upon from the meso-perspective, the RS-component of an organization is the collection of all tasks that can be identified at the microlevel. Its IS-component's primary function is to coordinate these tasks in order to improve the performance of the organization in its entirety. Its place in society, relationships with other organizations or markets are not taken into account. All such external relations are modeled in terms of communication with the IS/RS-combination's environment.

The macro-perspective serves to make these external relationships explicit, considering society (or part thereof) as an IS/RS-combination, and individual organizations as mere entities in its RS-component.

In brief, information systems, like the organization they are part of, are complex, multi-leveled, dynamic entities. The information paradigm serves to make this relation between information system and real system explicit at any level. Thus, the information paradigm stimulates us to look at organizations and information systems simultaneously. We feel that the design of information systems from this perspective requires new techniques for system description and analysis.

\subsection{RESEARCH QUESTION AND OUTLINE OF THE STUDY}

Our research question relates mainly to the second and third "megatrend" described in Section 1.1. We see that decision making is the central activity in organizations, and that we should focus on improving human performance. These trends hold strong implications for information systems design.

We emphasize that an information system is not an aim in itself. It purpose is to improve the performance of the organization it is part of. In formulating our preliminary research question we therefore at first do not speak of information systems. Instead, we consider an organization, its people, and their tasks.

When we consider the improvement of organizational performance from the micro-perspective, we see the need for supporting individual information workers. These people perform a variety of tasks, both structured and illstructured. This leads us to the first part of our research question:

1. How can we use problem-solving concepts in order to improve the performance of individual information workers in their workplaces? 
The second part of our research question relates to the meso-perspective:

2. How can we use problem-solving concepts to arrange suitable coordination between the tasks performed by these individuals, in order to improve the overall performance of an organization?

These two parts cannot be considered separately. There will inevitably be dependencies between workplaces (or organizational units, departments, divisions) because they are always part of some larger system. Moreover, these dependencies are not static. As we have seen at the beginning of this chapter, post-industrial organizations will be subject to more frequent and more radical changes, increasing the need for more frequent but also more complex decision making. Our aim, therefore, should be to develop an approach to improve organizational decision making. Once we have formulated this approach, we must investigate how we can support it with information technology. Thus, the third part of our research question is:

3. Can we create an environment for information workers that will improve their performance in solving both micro-level and meso-level problems, and

if so, what kind of facilities should such an environment provide?

We shall attempt to address these issues using an inductive-hypothetic research method (Sol 1982, page 4). In order to refine our research question we start with an investigation of the literature on problem-solving theories. In Chapter 2 we first justify why we restrict ourselves to theories based on the paradigm of bounded rationality, and subsequently define key concepts such and problem structure, task, decision, and coordination. We use our findings primarily to arrive at a more concise research question as part of what Lakatos (1970) would call a "research programme" for information systems design. The remaining chapters report on the way we have tried to answer this research question. In Chapter 3 we propose a single approach to micro-level and mesolevel problem solving. In Chapter 4 we outline an environment that supports thi approach with information technology. Next, we test the useability of this approach and the feasibility of the environment by performing a number of case studies (Chapters 5, 6 and 7). Finally, we evaluate our findings in Chapter 8 pointing out the strengths and weaknesses of the approach, as well as possible extensions.

\section{THEORIES ON PROBLEM SOLVING}

It is simply a truthful contradiction to me that people's problems are often funny and that the people are often and nonet

\subsection{INTRODUCTION}

In the previous chapter we have seen the strong relationship between information systems, organizational decision making and human problem solving. Thus, problem solving became the pivot of the tentative research question we formulated in Section 1.5. In this chapter we review a number of basic concepts from problem-solving theory. First, we describe what we mean by "problem", and consider different types of rationality. In Section 2.2 we look at problem structure, in particular at the difference between structured and ill-structured problems. In Section 2.3 we consider the meaning of the terms "task" and "decision". In Section 2.4 we look at the coordination aspect of problem solving in organizations. Finally, in Section 2.5 we refine our research question.

Various definitions of "problem" can be found in the literature, some of which are highly formalized (Mitroff and Betz 1972; Mitroff and Featheringhouse 1974). We adopt the rather informal one given by Ackoff (1981, page 20) primarily because it places humans at the center of problems and problem solving:

"By a problem we mean a situation that satisfies three conditions: First, a decision-making individual or group has alternative courses of action available; second, the choice made can have a significant effect; and third, the decision maker has some doubt as to which alternative should be selected."

We emphasize that according to this definition a problem requires a problem owner. Problems do not exist until some human perceives a situation as such Furthermore, this definition includes situations that are not "problematic" in the sense that the problem owner or owners resent that situation. Take for example problems that recur often, and for which a general solution has been found. Such problems have become "transactions", and problem solving has turned into transaction processing. Moreover, according to Ackoff's definition, an opportunity - a new, possibly advantageous situation - is also a problem.

Humans may seek to solve problems in different ways. Following Simon (1981, page 31-32) we distinguish between "substantive rationality" and 
"procedural rationality" as different perspectives on problem solving. True substantive rationality assumes perfect knowledge and a limitless computational capability. Such requirements are met only in artificial situations, abstractions of reality. When facing a real-life problem, the complexity of this problem usually exceeds the decision maker's computational capability. As problem complexity increases, problem solving gradually changes from choosing the right course of action to finding a way of determining what that course of action is. Procedural rationality primarily relates to this "procedure" a decision maker follows to arrive at $a$ solution, which is not necessarily the optimal one.

Procedural rationality is characterized by a problem specification that corresponds as closely as possible to reality, even if this means that such a specification offers no hints at all as to which alternative course of action is best. Procedural rationality is perhaps better known as "limited" or "bounded" rationality. We prefer this phrase as it reflects the reason why it was coined in the first place: the cognitive limitations of the human brain.

For completeness' sake we point out that in addition to substantive and procedural rationality, various other rationalities have been developed to explain observed behavior. We refer to March (1978) and Sage et al. (1983) for an overview.

\subsection{THE 'STRUCTUREDNESS" OF PROBLEMS}

In the previous section we have spoken of problems in general. We did not consider the Type I/Type II task dichotomy or the distinction between structured and ill-structured decisions mentioned in Chapter 1 . We shall presently try to define the concept of the "structuredness" of problems.

When addressing the issue of problem structure, information systems literature on problem solving most often quotes Simon (1977, page 23), who uses the terms "programmed" and "non-programmed":

Decisions are programmed to the extent that they are repetitive and routine, to the extent that a definite procedure has been worked out for handling them so that they don't have to be treated de novo each
time they occur. ... Decisions are non-programmed to the extent that they are novel, unstructured, and usually consequential. There is no cut-and-dried method for handling the problem because it hasn't arisen before, or because it is so important that it deserves custom-tailored treatment."

According to Amarel (1966), solving a problem simply means representing it so as to make the solution transparent. S $\phi$ lvberg (1975, page 411-412) articulates a similar statement. He calls a problem structured if it can be written down, documented, and handed over to a specialist, who will solve the problem without needing additional information. An ill-structured problem has no definitive formulation. No matter how the ill-structured problem is formulated, the problem solver comes up with a requirement for additional information. Every formulation of the ill-structured problem corresponds to a statement of the solution, and vice versa. To understand a problem is identical with solving the problem.

Mintzberg et al. (1976, page 246) use "unstructured" to refer to decision processes that have not been encountered in quite the same form, and for which no predetermined and explicit set of ordered responses exists in the organization.

We like to consider these definitions of ill-structuredness as "procedureoriented". Their essence is the novelty of the problem in the sense that the decision maker does not know what problem-solving procedure he should follow.

By contrast, the following definitions could be called "model-oriented". According to Young (1984), the degree of structure of a problem depends on how much is known of the following three basic components:

1. Objectives: in ill-structured problems not all objectives may be known at the outset; multiple objectives exist rather than one, and the trade-offs or relative utilities of the objectives are largely unknown.

2. Outcome-affecting variables: in ill-structured problems the identity of all of the important variables (both controllable and uncontrollable) that affect the outcomes may not be known at the outset of the decision process, and therefore complete models cannot be specified in advance.

3. Relations between affecting variables and outcomes: in ill-structured problems these relations are not all well-known in advance, or they may vary according to different plausible assumptions.

This characterization implies that one can speak of a structured problem when it can be expressed in terms of objectives, variables, and relations. Note the way Young emphasizes the relation between problem structure and the ability of the problem solver to create a model of the problem situation.

Sol (1982, page 5) makes this relation more explicit. He defines a problem to be structured if the following three conditions are met:

1. the set of alternative courses of action or solutions is finite and limited;

2. the solutions are consistently derived from a model of the problem situation that has been validated empirically and shows good correspondence with reality;

3. the effectiveness and/or the efficiency of the courses of action can be numerically evaluated. 
Problems that do not fulfil these requirements are defined as being illstructured. This definition relates the "structuredness" of problems not only to the degree to which the problem can be formalized (captured in a model), but also to the demands it imposes on the problem solver's computational capabilities.

Basically, the common element of the procedure-oriented definitions is the lack of some well-defined strategy - a programme* - according to which the problem at hand can be solved. In these definitions the link with bounded rationality is obvious. The model-oriented definitions do not characterize problem "structuredness" in terms of the problem-solving process, but consider the possibility to model the problem situation instead. To arrive at an unambiguous concept of "problem structuredness" we first wish to explicate what we mean by "problem structure".

We recall that we defined a problem as a situation in which a decision maker has alternative courses of action available, his choice can have a significant effect, and there is some doubt as to which alternative should be selected. Assuming that this situation can be described in terms of a - possibly large and not completely identifiable - set of related variables, the alternative courses of action can be viewed as operations on variables. As the decision maker's choice can have a significant effect, each operation will change the current state of some subset of these variables (and possibly some other variables as well), depending on the relations between these variables. The decision maker's doubt is caused by the circumstance that - according to that decision maker - some states may be more favorable than others. In other words, the decision maker must have some evaluation criterion. According to this view, the structure of a problem consists of these variables, relations, operations, and evaluation criteria.

Daft and Lengel (1986, page 556) relate uncertainty to what we call "problem structuredness". They make a distinction between two types of uncertainty. First, there is the "common" uncertainty which is defined as the absence of information, a definition that is traced back to Galbraith (1977), who defines uncertainty as the difference between the amount of information required to perform a task and the amount of information already possessed by the organization. Secondly, there is "equivocality", the existence of multiple and conflicting interpretations about an organizational situation (Weick 1979).

We can easily describe these two types in the terms we used to define problem structure: uncertainty refers to the situation in which the problem structure - in particular its variables - may be known, but not the current state

"Note the distinction between problem-solving programme and computer program. of (some of) its variables. Equivocality refers to uncertainty about the problem structure: its variables, relations, operations, evaluation criteria, or any combination of these.

Supposing that the "structuredness" of a problem is the degree to which its structure is known, we could use the term "equivocal" instead of "ill-structured". If the decision maker knows exactly which variables to consider, if he is aware of all the relations between these variables, if he can predict with certainty what the result of his operations will be, and if he knows which evaluation criterion to apply, the problem is structured. However, in the previous section we mentioned that perfect knowledge of the problem structure in combination with ample computational capability occur in artificial situations only. In reality there always are computational constraints, and problems always involve some degree of uncertainty and equivocality. This would mean that in real situations we can never speak of structured problems! For this reason we prefer "structured" in the sense that a problem-solving programme is known to "structured" in the sense that a model of the problem exists.

Having taken our position, we stress that no problem is de facto structured or ill-structured. The process of solving an ill-structured problem requires structuring this problem, a statement that can be found at many places in the literature (Mintzberg et al. 1976; Van de Ven 1980; Ackoff 1981; Van Gundy 1981). For this reason, Maes et al. (1983, page 140) distinguish between "a prior structured" and "a priori ill-structured" problems. By specifying a programme according to which an a priori ill-structured problem should be dealt with, this problem is converted into an a posteriori structured problem. Structured, not in the sense that the decision maker knows the problem structure, but in the sense that - using the words of Huber and McDaniel (1986a, page 585) - he has "formally decided what to decide". This way of "structuring" a priori illstructured problems is inherent in bounded rationality.

Various representations of problem-solving programmes exist. Joyner and Tunstall (1970), Bosman (1986), and Van Schaik (1988) use flowcharts, Hackathorn (1977a, b) employs a variant of Petri nets, while Maes et al. (1983) use decision tables. An expert system's rule base is in fact also such a representation. In other words, the essential difficulty in solving an a priori illstructured problem is not how to represent an appropriate programme. The difficulty is how to design it. Naturally, this difficulty may depend on the extent to which the problem structure is known.

Although we consider only the second one in determining the "structuredness" of a problem, problem solving still has these two sides: analysis of the problem structure, and design of the problem-solving strategy. In the remainder of this section we will show how these two aspects become apparent in the problem solving process. Sol $(1982,1985)$ gives a general outline of the process of 
problem solving, based upon Mitroff et al. (1974). He puts great emphasis on problem definition, first in broad terms in order to establish the general nature of the problem and the decision maker's goals (conceptualization), then in detail, aiming for good correspondence with reality (specification). Solution finding should depart from this problem definition. Thorough conceptualization and specification reduce the risk of making an "error of the third kind". that is to say, the risk of solving the wrong problem (Mitroff and Featheringhouse 1974).

In our terminology, conceptualization and specification correspond to the analysis of the problem structure. This analysis leads to a model of the problem situation. We emphasize that the primary aim should be to maintain a good correspondence with reality. Note that even if a problem situation can be modeled adequately, and if the problem can be diagnosed, this problem may yet be "novel" in the sense that an algorithm to generate and test alternatives still has to be designed. If such an algorithm can be designed, each alternative can be evaluated, and the one that seems most advantageous can be chosen.

However, if the problem situation can not, or only partially, be modeled, or if the complexity of the generate-and-test algorithm exceeds the decision maker's computational capability, solution finding will be fundamentally different. Instead of looking for an optimal solution, it will consist of designing and testing programmes - heuristic rather than optimizing - and choosing the one that seems most appropriate. Again, tests are performed using the (possibly incomplete) model of the problem situation.

The gist of this section is that there are two sides to the "structuredness" of problems. One relates to the what: problem models. The other to the how: problem-solving programmes. We favor the second aspect, and therefore reserve the qualification "ill-structured" for those problems for which no programme has been found so far. Yet both aspects are of great importance in problem-solving processes. Therefore, if we wish to support problem solving, we need a set of concepts in terms of which problem models and problem-solving programmes can be expressed.

\subsection{DECISION AND TASK}

In order to be consistent in our terminology we need a proper definition of the notions "decision" and "task" and their relation to "problem" and "problem structure". First, we look at the concept of "decision". Little (1971, page 467) justly remarks that a decision is usually a murky event, identifiable only in retrospect. A reason for this vagueness may be that decision can be interpreted as a distinct choice (Tverski and Kahneman 1974; Holloway 1979; Pitz 1981), but also as a process (Simon 1977; Miner 1979; March and Shapira 1982; Einhorn and Hogarth 1986).

Problem solving and decision making are closely related and often used as equivalent terms. Clearly, solving what Ackoff defines as a problem (see Section 2.1 on page 13 ) requires decisions to be made. The decision maker - mark the word - has to decide which of the alternative courses of action he should follow.

Recently, Simon et al. (1987, page 11) described the work of making decisions and solving problems as work of choosing issues that require attention, setting goals, finding or designing suitable courses of action, and evaluating and choosing among alternative actions. In their view, the first three of these activities - fixing agendas, setting goals, and designing actions - are problem solving, while the last - evaluating and choosing - is decision making. Intuitively, decision making is closer to the notion of "choice" than is problem solving.

In earlier work Simon (1977, page 40-41) uses problem solving and decision making as equivalent terms. He distinguishes three major phases in the decisionmaking process:

- Intelligence: searching the environment for conditions calling for decisions. Raw data are obtained, processed, and examined for clues that may identify problems.

- Design: inventing, developing, and analyzing possible courses of action. This involves processes to understand the problem, to generate solutions, and to test solutions for feasibility.

- Choice: selecting a particular course of action from those available.

This characterization of decision-making processes includes all of the four activities: fixing agendas, setting goals, designing actions, and evaluating and choosing. In other words: depending on one's point of view, the question whether decision making and problem solving are equivalent can be confirmed as well as denied.

Since the purpose of this section is to provide a consistent terminology, we must choose a single perspective. Essentially we prefer the view expressed by Simon et al. (1987). If the set of alternatives has been identified, requiring evaluation and choice only, we would like to speak of a decision. But when intelligence and/or design activities are still required we would rather speak of a problem. But what if evaluating and choosing itself requires intelligence or design? Simon (1977, page 43) explicitly describes this interweaving of the three phases: 
"Generally speaking, intelligence activity precedes design, and design activity precedes choice. The cycle of phases is, however, far more complex than this sequence suggests. Each phase in making a particular decision is itself a complex decision-making process. The design phase, for example, may call for new intelligence activities; problems at any given level generate subproblems that, in turn, have their intelligence, design, and choice phases, and so on. There are wheels within wheels within wheels."

The distinction between problem and decision implies that decision making is part of problem solving. But the observation that each decision-making phase requires problem solving would imply that problem solving is a part of decision making. Fortunately, this only seems to be inconsistent. Note that Simon speaks of problems at different levels. At each level we can see decision making strictly as a part of problem solving. Only when simultaneously considering problems at different levels should we be careful with our terminology.

In addition to the three major decision-making phases, Simon mentions a fourth (minor) phase, "review", which comes down to assessing past choices. But he also defines this fourth phase as the task of carrying out decisions (Simon 1977, page 43). This second interpretation is most widely cited as the "implementation phase". Since implementation of a chosen alternative generates subproblems in much the same way the other three phases do, this additional phase does not affect our reasoning.

Note that in this reasoning we have not referred to the description of the process of problem solving we described in Section 2.2. We did not make this reference as in our opinion the views of Simon and Sol are equivalent. We are aware, though, that there is a distinct difference in emphasis. Both identify an intelligence phase, but Simon is rather unspecific on the issue of problem identification and formulation, while Sol stresses the importance of thorough conceptualization and specification. Both identify a solution finding activity, but the moment of choice is much more prominent in Simon's model than in Sol's.

The second concept we wish to investigate in this section is "task". Intuitively, a task is something that has to be performed in order to achieve a certain goal. We like to consider any task as a problem-solving process. It would seem logical to say that a task is structured if the problem it deals with is structured, and ill-structured otherwise. But the distinction is not that straightforward, as again we should distinguish between a priori structured and a posteriori structured.

We can state that if the problem at hand can be modeled, and if there is some formal theory or algorithm that guarantees that an optimal solution can be found within a reasonable amount of time, the problem-solving process (the task) is structured. We might add that when such a task is decomposed into subtasks, each of these subtasks will correspond to specific parts of the theory or steps of the algorithm, meaning that each subtask is also structured.

But if there is no theory or algorithm, the decision maker will have to design a programme according to which he will arrive at a solution. By formulating this programme the a priori ill-structured problem becomes a posteriori structured. The problem structure, however, remains only partially specified. We therefore speak of structuring the task, rather than structuring the problem. In our view, the programme is the "task structure".

Tasks are often defined recursively in terms of subtasks, meaning that tasks can be decomposed into a hierarchy of subtasks until a desired level of detail has been reached. There are some disadvantages to this view, which we shall discuss in Section 3.3. For the moment we only wish to point out that a good method for task specification should provide means to represent tasks in a descriptive as well as in a prescriptive way. A purely descriptive task representation contains information only on what is to be done and not how it should be done, meaning that it cannot express the procedural aspect of solving a problem. On the other hand, a strictly prescriptive task representation cannot depict the problem structure as defined on page 16 .

By stating that every task corresponds to a problem-solving process we have defined the relation between problem and task, and by viewing decision making as a distinct phase of a problem-solving process, we implicitly defined the relation between decision and task. Evidently, the recursive definition of a task in terms of subtasks fits the recursive nature of a problem. If solving a problem $\mathbf{P}_{0}$ constitutes task $t_{0}$, then solving a subproblem $\mathbf{P}_{\mathrm{i}}$ that is generated during the intelligence, design, choice, or implementation phase constitutes a subtask $t_{\mathrm{i}}$ of task $t_{0}$.

Basically, what we have said in this section can be summarized in four observations, or perhaps we should say "fundamental assumptions", since they constitute the basis for our further reasoning:

1. every task corresponds to a problem-solving process;

2. every problem-solving process is a - possibly very complex - sequence of decisions;

3. a programme specifies how a task is divided into subtasks, and which decisions have to be made, and in which order (note that by order we do not rule out that some decisions may be made in parallel);

4. if a programme is known, the problem or the task that solves it is structured, and ill-structured otherwise. 


\subsection{THE COORDINATION PROBLEM}

Child (1977) defines organizational structure as the allocation of tasks and responsibilities to individuals and groups within the organization, and the design of coordinating mechanisms to ensure effective communication and integration of effort. Mintzberg (1983, page 2) rejects the idea that there is one best way to design the structure of an organization. He sees this design as a problem that continuously recurs. A problem because there are two fundamental and opposing requirements: the division of labor into various tasks to be performed, and the coordination of these tasks to accomplish the activity. We shall refer to this problem as "the coordination problem".

Mintzberg (1983, page 4-7) identifies five coordinating mechanisms that seem to explain the fundamental ways in which people within organizations coordinate their work:

1. Mutual adjustment, based on informal communication;

2. Direct supervision, meaning that one person takes responsibility for the work of others, issuing instructions to them, and monitoring their actions;

3. Standardization of work processes by specifying (programming) the contents of the work

4. Standardization of outputs by specifying the results of the work;

5. Standardization of knowledge and skills by specifying the kind of training required to perform the work.

These coordinating mechanisms appear to fall into a rough order. Mintzberg finds that as organizational work becomes more complicated, the favored means of coordination seems to shift from mutual adjustment to direct supervision to standardization. Standardization of work processes is preferred to standardization of outputs, which is favored over standardization of skills. This is not to say that these coordinating mechanisms are mutually exclusive! Solving the coordination problem means choosing the combination of mechanisms that is best suited to the situation. As this situation may change constantly, the coordination problem definitely has a recurring nature.

Each coordinating mechanism can be described in terms of the information paradigm we presented in Section 1.4. The basic assumption we make when applying this paradigm to tasks and their coordination is that a task can be viewed as an IS/RS-combination, and that coordinating mechanisms can be seen as different applications of the recursion principle, or as different architectures of the IS-components.

In the case of mutual adjustment, coordination is achieved through communication between the IS-components of the two IS/RS-combinations, as is shown in Figure 2-1(a). Mutual adjustment can be described without recursion, as each IS/RS-combination views the other as part of its environment. Each IS exerts control over its RS according to the information it obtains from this RS and the information that is provided by the other IS. Note that this kind of coordination requires considerable flexibility from the part of both ISs.

Direct supervision is the classical example of the recursion principle. Two or more IS/RS-combinations are considered as one RS, and the corresponding higher level IS issues instructions to the lower level ISs and monitors their actions, as is depicted in Figure 2-1(b). Each lower level IS will be considerably less complex than a mutually adjusting IS would have been, since it only has to interpret the instructions issued by the higher level IS. At the same time the higher level IS needs to know less about the lower level RSs as their ISs handle the details.

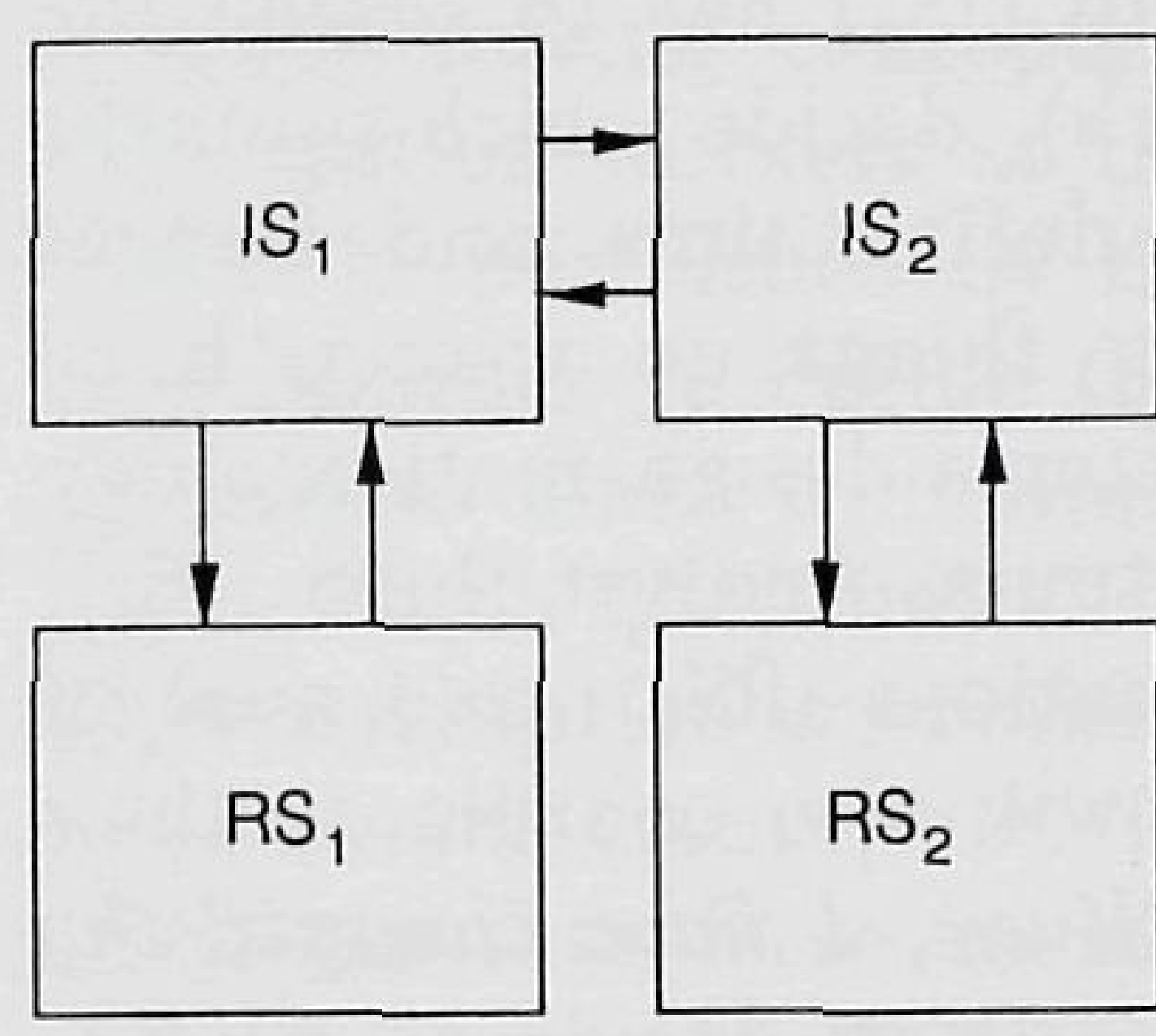

(a) Mutual adjustment

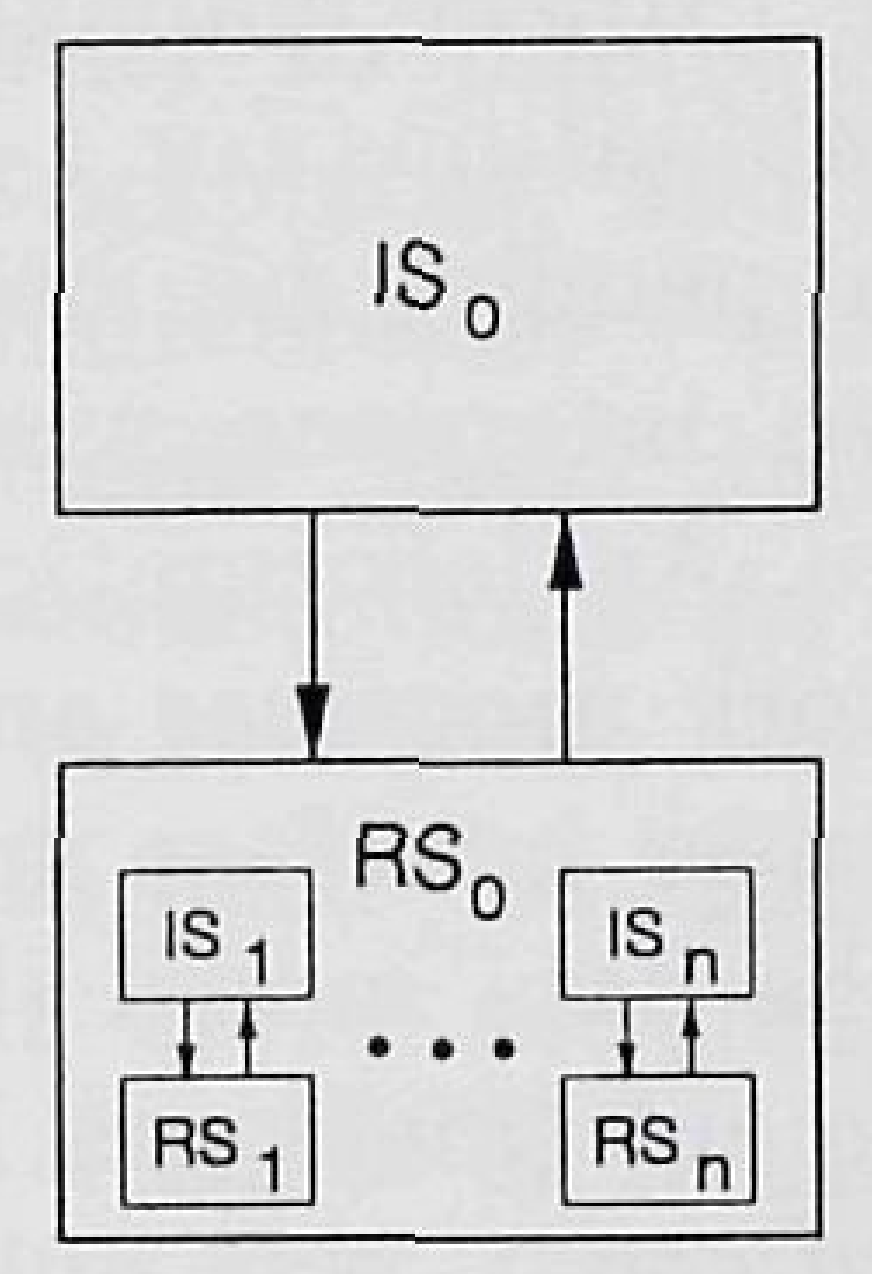

(b) Direct supervision

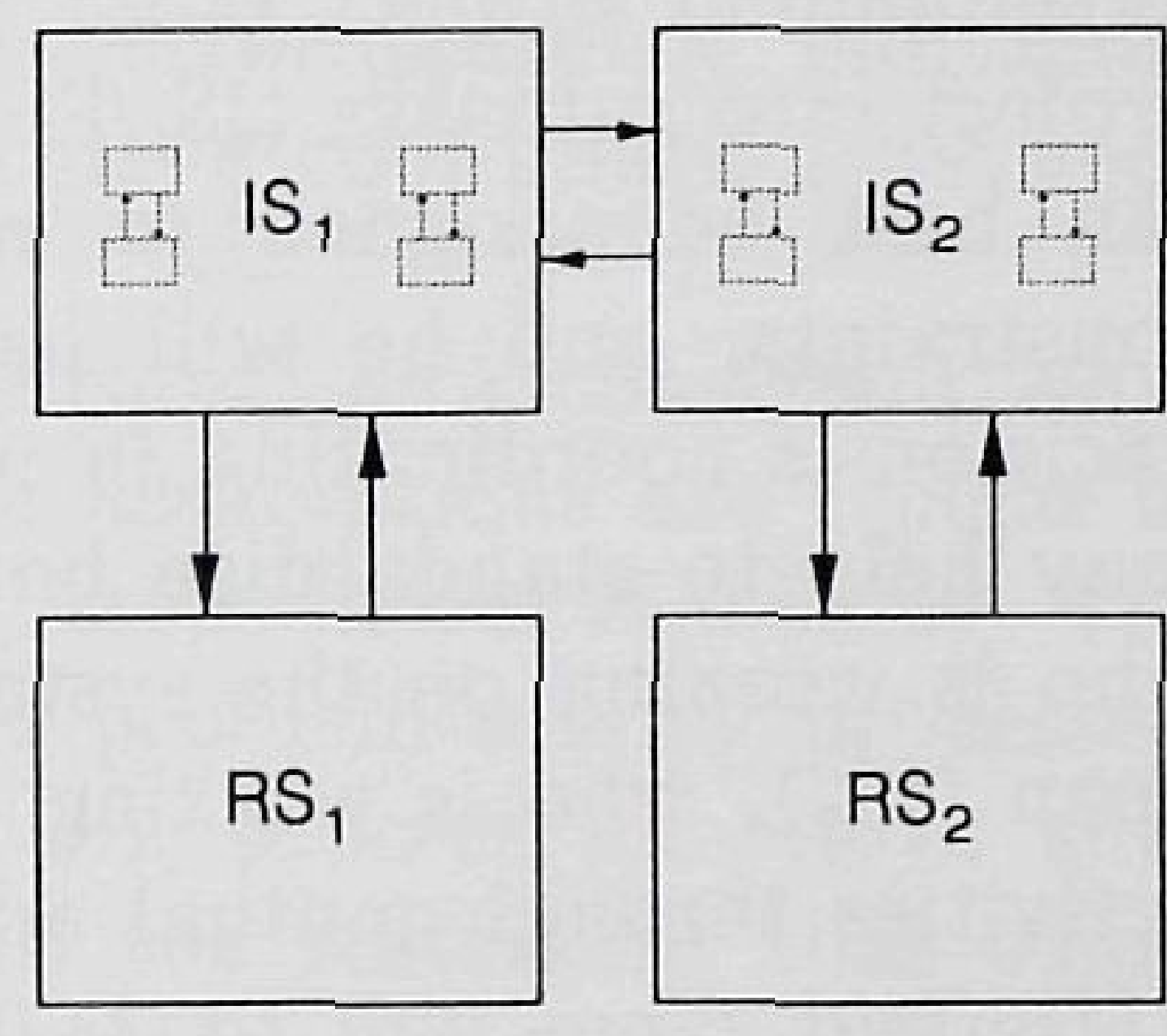

(c) Standardization of skills
Figure 2-1. Three coordinating mechanisms in terms of IS/RS-combinations

Standardization of work processes achieves coordination through exact specification of the way the IS controls the RS. Note that this corresponds with the prescriptive task specification we mentioned on page 21. Similarly, when outputs are standardized, the IS is "programmed" in terms of what should be done instead of how it should be done, which corresponds to descriptive task specification. Thus, the difference between these two coordinating mechanisms lies in the architecture of the IS rather than the way the recursion principle is applied - a difference that is difficult to depict graphically.

This also holds for the fifth coordinating mechanism. Standardization of knowledge and skills achieves indirectly what standardization of work processes or work outputs does directly. Mintzberg (1983, page 6-7) uses the following example: When an anesthesiologist and a surgeon operate together, they hardly need to communicate. By virtue of their training, they know exactly what to expect of each other; their standardized skills take care of most of the coordination. In other words, each IS has in addition to knowledge related to the task it is part of, also knowledge of what the other ISs know, that is to 
say, the way the other tasks will be performed. This knowledge, which is essentially a collection of models of the other IS/RS-combinations (depicted in Figure 2-1(c) as “品”), reduces the need for communication, but it increases the complexity of the IS.

In the previous section we defined a task as a problem-solving process. Here we define it as an IS/RS-combination. The two definitions combine very well if we take the position that a decision is always made within an IS, while the consequences of this decision can be seen in the corresponding RS as changes in some of its variables. As the IS collects data from the RS concerning the state of its variables, these changes can indeed affect other decisions.

As an example, consider the task of getting some information systems development project $\left(\mathrm{RS}_{0}\right)$ done. Project manager Pete $\left(\mathrm{IS}_{0}\right)$ has to divide the project into subtasks $\left(\mathrm{IS}_{\mathrm{i}} / \mathrm{RS}_{\mathrm{i}}\right.$-combinations for $\left.\mathrm{i}=1, \ldots, \mathrm{n}\right)$, decide which subtasks can best be assigned to which people, he has to define time and budget constraints, and he will have to intervene whenever things go wrong. Each decision is coordinating in nature. An information systems design methodology may help to standardize both work processes and outputs. Analyst Huey ( $\mathrm{IS}_{1}$ ), who is working on the system's user interface specifications $\left(\mathrm{RS}_{1}\right)$, and analyst Dean $\left(\mathrm{IS}_{2}\right)$, who is working on the data model $\left(\mathrm{RS}_{2}\right)$, will also coordinate their activities through mutual adjustment (messages like "Huey, I have changed the PID field from 9(6) to X(10). Dean"). Not only does Dean decide to change the PID format, he also decides that it is necessary to inform Huey about thi change.

Thus we persist in our opinion that a task is essentially a collection of decisions. Performing a task is decision making - any task, even sharpening one's pencil, since implementing a decision may involve physical actions. If coordination between tasks is required, this often means more decision making. This is particularly evident in the case of direct supervision, where the coordination is achieved by adding a task - the supervisor task - that is purely coordinating in nature.

Summarizing this section we can say that the coordination problem appears when a problem is complex to the extent that the task of solving this problem has to be divided into subtasks that are to be performed by different decision makers. Mintzberg defines coordination as choosing appropriate coordinating mechanisms. We have shown that these mechanisms can be expressed in terms of decision making. In our opinion, choice of appropriate coordinating mechanisms is equivalent to design of an appropriate task structure. Thus, solving the coordination problem is always part of solving the original problem.

\section{RESEARCH QUESTION REVISITED}

In the preceding sections we have presented a theoretical view on problems and problem solving. We have given an informal definition of the key concepts "task" and "decion". Finally, we have described our interpretation of the inction of the coordination problem in terms of these concepts, and indicated its importance in organizational problem solving.

In this section we reconsider the tentative research question we formulated in Section 1.5. There we stated that the overall issue is the improvement of organizational performance, but that we should begin by improving the primary process (Sol 1989). Developments in the DSS and OIS area induced us to consider the support of information workers from a problem-solving perspective. We therefore formulated our preliminary research question as how to apply problem-solving concepts in order to improve the performance of individual information workers at their workplaces, and to achieve coordination between the tasks performed by these individuals.

In order to define the concepts "task" and "decision" in a consistent way, we had to make a number of assumptions. As these assumptions are crucial to our research question, we wish to recall the essential steps in our reasoning. The first assumption we make is that tasks correspond to problem-solving processes. Our second assumption is that every problem-solving process generates subproblems that have to solved. This is equivalent to the statement that every task has subtasks that have to be performed. The third assumption we make is that we should distinguish between problem solving and decision making or, what is the same thing, between problem and decision. We define problem solving to include what Simon calls the intelligence, design, choice, and implementation phases, while decision making refers to the choosing between alternatives only. Our final assumption is that when describing a problemsolving process, information can always be related directly to decisions.

In the light of our first three assumptions we may conclude that every task involves at least one decision. If it has subtasks, there will be more decisions, as each subtask involves at least one decision as well. Our fourth assumption allows us to consider the flow of information in relation to problem solving. The intelligence and design phases are viewed basically as information gathering and ordering, and generation of alternatives. The obtained information, which consists of alternatives and evaluation criteria, serves as "input" for the choice phase. The "output" of the choice phase is also information - the chosen alternative which is used in the implementation phase.

We argued that solving the coordination problem is part of every problemsolving task, and that coordinating mechanisms can be specified in terms of decisions in much the same way as problems involving but a single decision maker. Although the coordination problem makes the design of an appropriate task structure more complex, the idea of specifying a problem in terms of decision processes remains valid. 
In essence this idea is not new. Simon (1977, page 123) points out that the main responsibilities of managers will be for the maintenance and improvement of the "decision system", and that managers will spend much of their time and effort in analyzing and designing policies and the systems for implementing them. Hackathorn (1977b), Sol (1982, page 16), Stabell (1983, page 228), Bosman and Sol (1985, page 90), and Todd and Benbasat (1987, page 494) all argue that decision processes must be specified in order to obtain a better insight, and eventually to make better decisions. Huber (1984a, page 936) expects that both the increased accessibility of information and the "routinization" of decisions will lead to formalization of decision processes. This calls for the management of decision-making processes, since these are regarded as central activities that should not be left to chance (Huber and McDaniel 1986, page 585).

In this thesis we elaborate part of what Lakatos (1970) would call a "research programme" for the improvement of organizational performance by means of information technology. If we adopt his terminology, the "hard core" of this research programme consists of two "irrefutable" theories:

1. the performance of an organization is directly related to the decisionmaking capabilities of its individual workers and the coordination between these individuals;

2. research into organizational information systems should be based on the concept of bounded rationality.

The "protective belt" of our research programme basically consists of five hypotheses. Three of these relate to the specification of problem-solving processes; the fourth and fifth bring the design of information systems back into focus:

1. in the process of problem solving one should distinguish between problem structure and task structure;

2. tasks and coordinating mechanisms can be specified in terms of decisions that are to be made;

3. decisions can be specified in terms of the information they require and generate;

4. the construction of conceptual and empirical models, and the design and analysis of task structures can be adequately supported with information technology that is currently available;

5. every task structure constitutes a "blueprint" for a task-specific information system.

These five hypotheses together constitute a "refutable" theory that offer guidance in fulfilling what Sprague (1986, page 19) calls "the ultimate mission

26 of information systems" - to improve the performance of information workers through the application of information technology. If this theory is not refuted, through the application of information technolich we can state that it is possible to create an environment ince the focus is on makers can solve problems faster and more effectively. Since the focus is on problem solving, with information technology in a supporting role, we avoid the issue of discriminating between MIS, DSS, OIS, ES, EIS, or whatever other class issue of discriminating between 列 while MIS, DSS, ef information technology. This gap can be bridged if we refer to the abstract notion as the "information system in the broader sense", and to the concrete applications as "information systems in the narrow sense" (Verrijn-Stuart 1989). Task structures, being flexible specifications of problem-solving programmes that can structures, being flexible specifications of problem-solving programs, constitute be designed or modified to cope with new (coordination) problems, constitute means for describing and structuring an information system in the broader sense, while the task-specific information system mentioned in the fift hypothesis of our theory is an information system in the narrow sense.

So far we have expressed our theory in a very informal way. It will be So far we habsequently, it is tested for feasibility and formulated explicitly in Chapter 3. Subsequently, it is tested for feasibility and applicability. In Chapter 4 we investigate what kind of computerized support is needed in order to apply the theory, and demonstrate that the construction of
this support is feasible. The applicability of the theory is tested by conducting three case studies.

Chapter 5 reports on our first case: the problem of shiftwork planning. The purpose of this study is to test whether the problem-solving process of a single decision maker can be described adequately in terms of tasks and decisions, and dhether this description constitutes a sound basis for the development of adequate decision support.

The objective of the second case study - an analysis of the problem of international transfer pricing, presented in Chapter 6 - is to test whether our theory helps to solve the coordination problems that arise when a team of theory helps to solve the coordination makers has to solve one particular problem.

The third case study, which will be described in Chapter 7, is based on a management game. A management game requires players to make a variety af manalial decisions while abstracting from the lowest of managerial decisions while abstracting from the detalls at the low operational level of the simulated organization. Thus, it allows investigation of typical meso-level coordination problems within a relatively narrow time frame. Moreover, since its users manual constitutes a fairly detailed problem statemen (it specifies what the players have to do, but not how), a management game makes it possible to test whether our theory allows a smooth transition from problem statement to task structure. 


\section{SUPPORTING PROBLEM SOLVING}

Every problem becomes very childish when once it is - Sherlock Holmes

\subsection{INTRODUCTION}

In Chapter 2 we defined what we mean by "task" and "decision". In this chapter we shall relate these concepts to the process of problem solving. In Section 2.2 we gave an outline of this process. We now shall describe this view on the problem-solving process in detail.

According to Sol (1985, page 85), problem solving in general comprises four major "activities": conceptualization, specification, solution finding, and implementation. In Figure 3-1 these activities are depicted as transitions between four specific "stages" in the problem-solving process.

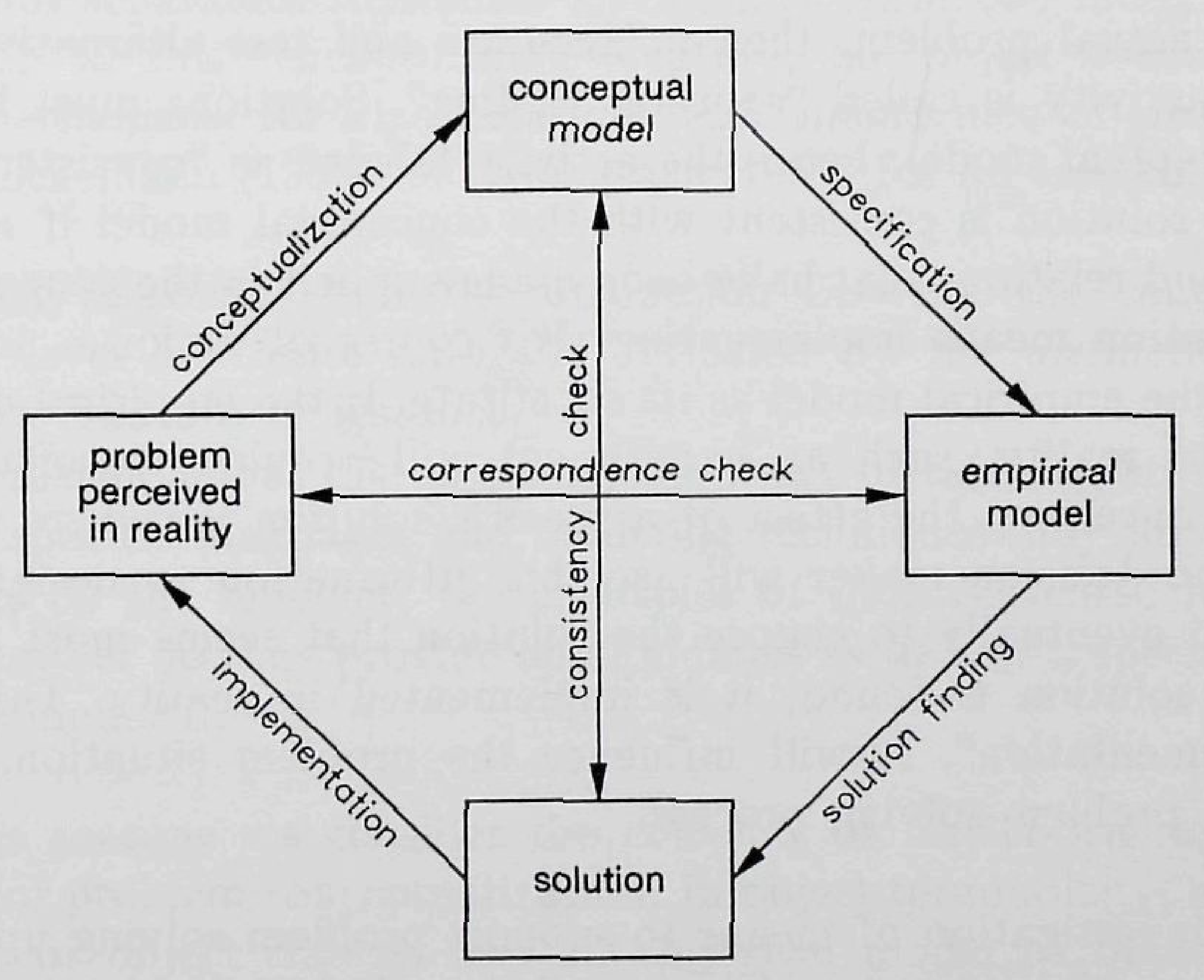

Figure 3-1. The process of problem solving

First, a particular problem is perceived in reality. In terms of our definition of "problem" on page 13, this means that a decision maker has alternative courses of action available, each of which will affect the problem situation. The decision maker is in doubt as to which alternative will produce the most favorable result. He even may not be aware of all possible courses of action. 
By "perceive" we mean that the decision maker constructs some mental model of the problem situation. This mental model provides him with the concepts in terms of which he can express the actual problem and think about possible solutions. It is therefore referred to as the "conceptual model" of the problem, and the activity that leads to this model is called "conceptualization".

Once formulated, the conceptual model provides the vocabulary to describe the problem situation in detail. This activity is called "specification". The primary purpose of problem specification is to represent the problem situation in such a way that the actual problem can be diagnosed correctly. This means that the result of the specification activity must model the relevant aspects of the problem situation. Such a description is called an "empirical model", as it must be tested empirically. In Figure 3-1 this test is called "correspondence check", and we shall refer to the measure in which the empirical model mirrors reality as "the degree of correspondence". Note that empirical models will be based on sample data, whereas conceptual models are data-void.

The loop "conceptualization-specification-correspondence check" usually mus be followed repeatedly before an empirical model that shows satisfactory correspondence has been arrived at. This empirical model is used first to diagnose the actual problem, then to generate and test alternative courses of action. This activity is called "solution finding". Solutions must be consistent with the conceptual model, hence the activity labeled as "consistency check" in Figure 3-1. A solution is consistent with the conceptual model if it refers only to variables and relations that have been made explicit in the conceptual model. Testing a solution means implementing that course of action - not in reality, but by using the empirical model as its substitute. If the empirical model closely corresponds to reality, such an experiment will provide reasonably accurate information concerning the effect of a specific solution if it were implemented in reality. The decision maker will use this information to design alternative solutions, and eventually to choose the solution that seems most appropriate.

When a solution is found, it is implemented in reality. This activity is called "implementation". It will influence the problem situation, which may induce a new problem-solving process.

We base our investigation of means to support problem solving upon this view on the problem-solving process. The remainder of this chapter is organized as follows: In Section 3.2 we propose a method to construct a conceptual model. In Section 3.3 we describe how this conceptual model can be translated into an empirical model. Special attention will be paid to the role an empirical model has to play in the solution finding activity, and to the demands this imposes on the manner in which solutions should be formulated. In Section 3.4 we describe a method for the formulation and analysis of solutions, based on the concepts task and decision, and indicate how it meets these demands.

\section{PROBLEM CONCEPTUALIZATION}

A conceptual model offers the concepts in terms of which the decision maker A conion actual problem he perceives. describes a problem sis language should To express these concepts a language is impose no restrictions with respect to the types of problem situation that can be described with it. This freedom is offered by natural languages, being the common vehicle for human thought. The difficulty is that a translation from a conceptual model in natural language to an empirical model that can be "run" like a computer program (a necessity if this model is to be validated using large lis reason, numerous formalisms for the construction of conceptual models have been developed.

In Section 1.3 we pointed out the growing popularity of object-oriented modeling. We see three main reasons for this development:

1. First there is the appeal of the concept "object" itself. When defining an object, one has to distinguish between "attributes" and "actions", but these two aspects - thinking of objects in terms of what they are and what they $d o$ - are not separated. Attributes and actions form one integrated entity. In addition to this "encapsulation" feature, the object concept provides elegant mechanisms for classification, with inheritance of properties. We elegant mechanisms for classification, with (1989) for an assessment of these refer to Lockemann (1989) and Nierstrasz (1989) for an assessm features.

2. The second reason is that the distinction between the conceptual and empirical level are quite naturally reflected by the distinction between object class and object instance.

The third reason is that the object-oriented way of thinking is very flexible. Although specific languages and modeling techniques may impose specific constraints on the modeler, the principles of object-oriented modeling do not. But neither do they provide any guidance as to how a specific situation should be modeled.

For these same reasons we consider the concepts of object-oriented modeling very suitable for problem conceptualization. In object terminology, a conceptual model consists of object classes, and an empirical model of object instances. Conceptualization means identifying object classes in reality, and defining their actions insofar as they are considered relevant to the problem. attributes and actions insofar as they are considered constiture the "vocabulary" with which a problem situation can be described.

Throughout this thesis we will use a very general, "non-committing" language to define object classes. An existing language, such as Smalltalk (Goldberg and Robson 1983), Beta (Madsen 1987), or one of the object-oriented derivatives of Lisp (Moon 1989) or C (Cox 1986; Stroustrup 1986) would draw 
attention to the specific details of the syntax of the language, which are irrelevant to our purpose. We therefore prefer to use the following notation for object class definitions:
First attribute

:

Last attribute

\section{actions}

first.action

last action ]

In other words, we denote specific object classes by upper case strings (for the moment we do not consider object instances), we refer to attributes by means of italicized lower case strings that start with a capital letter, and we represent actions as plain lower case strings. Whenever an explanation of attributes or actions seems appropriate, this is given in small Roman print. To clarify the practical meaning of terms, and to illustrate the use of techniques throughout this chapter, we consider one particular problem situation: route planning of a transportation firm.

In the course of problem conceptualization, four activities can be distinguished:

1. Identification of an object class: One or more similar objects are perceived as part of the problem situation, and potentially relevant to the problem at hand. As a category they are given a name and a verbal description. In the route planner's case we can for example identify VAN as the firm's only means of transportation.

2. Specification of an object class: The characteristics of these objects are defined in terms of attributes and actions, resulting in object class definitions like:

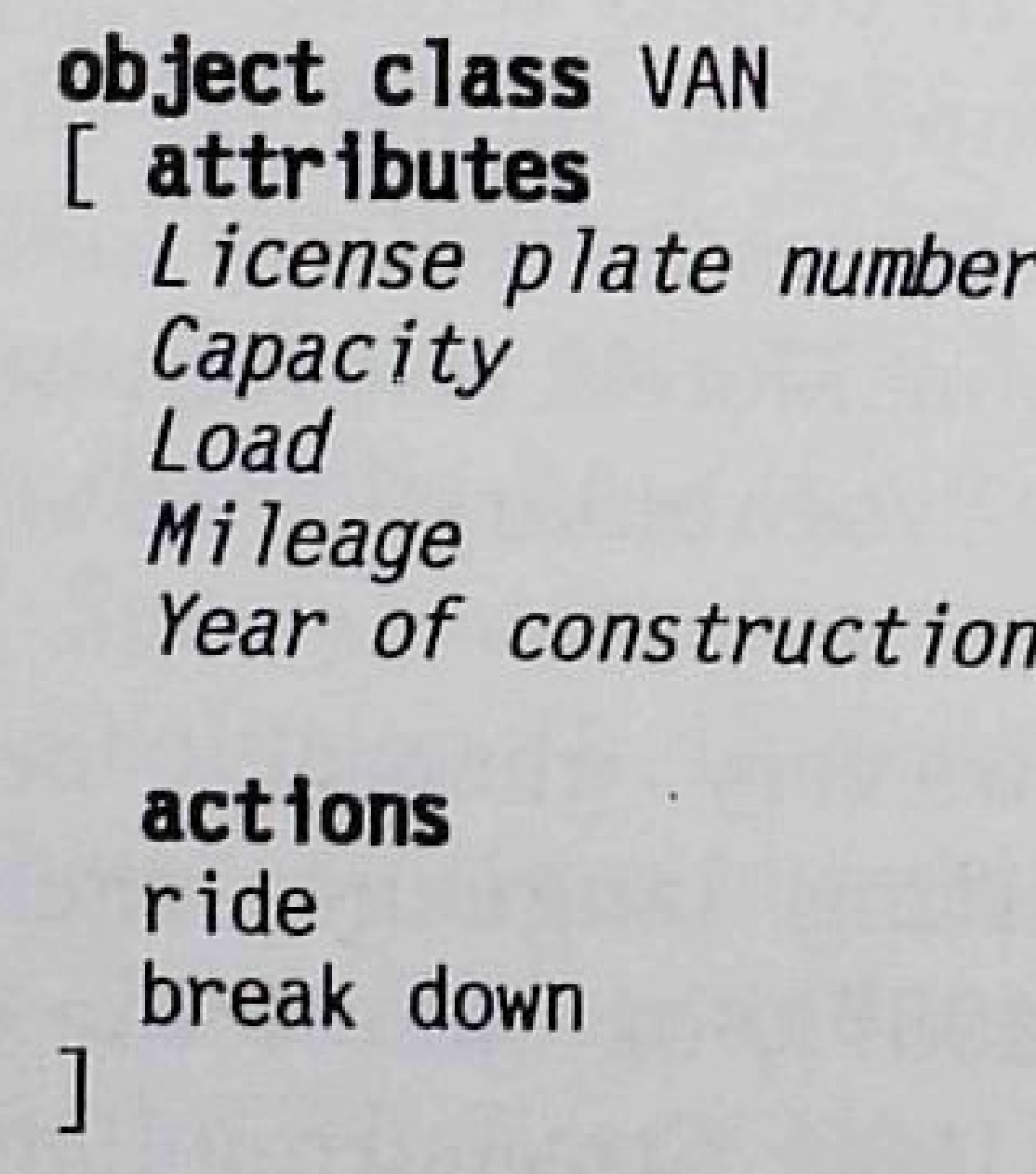

object class DRIVER
[ attributes
Name
Skill level
Region
actions
drive
deliver a parce l
take a break
replace a flat tire
]

\section{object class NAME \\ attributes}

3. Relating an object class to other object classes: Indicate for each object class which of its actions affect other objects (possibly instances of previously defined object classes), for example by changing their attribute values or by triggering their actions. Thus, a driver will change a van's load by delivering a parcel, and by breaking down, a van will cause itself to cease running.

4. Demarcation of problem boundaries: Determine which object classes, attributes and/or actions are truly relevant to the problem. If break-downs are rare, this action, and probably also the van's year of construction, will turn out to be irrelevant to the problem of finding the shortest route among a van's destinations.

There is no specific order to these four activities. During conceptualization they will be performed in constant alternation. Using our route planner's problem as an example we shall illustrate this process in more detail.

We first identify the object classes that are "directly visible", such as TRANSPORTATION FIRM, CUSTOMER, ROUTE PLANNER, VAN, DRIVER, and ROAD. Specification and identification often trigger each other. The fact that a van breaks down every now and then leads to the identification of the attribute Breakdown chance and the action break down of the object class VAN. But to specify the consequences of a van breaking down we identify the attribute Operational vans of TRANSPORTATION FIRM. Specifying and interrelating object classes also leads to the identification of new objects. Some may be obvious (a van needs a driver), others are less apparent (the time it takes to cover a specific stretch of road - an attribute of ROAD - will be affected by for example some instance of class TRAFFIC JAM on that road).

When determining the problem boundaries one has to decide when to stop adding more details. The information paradigm offers some guidance here. First it should be determined for all objects whether they are part of the IS, the RS, or the IS/RS-combination's environment. Those objects and/or attributes that are viewed as part of the environment need not be detailed. As an example, consider the way the break down action of a van is modeled. Since this aspect is absolutely beyond the control of the route planner, it is expressed by an attribute Average breakdowns per 10,000 kilometers. If the problem were not to plan routes but to consider buying new vans, breakdowns would have to be described in more detail, for example by relating them to a van's age and maintenance record.

A conceptual model is more than just a set of object classes in terms of which the problem situation can be described. It should also include some global formulation of the problem. As we stated in Chapter 2, we can speak of a problem only if there are several ways to change the current situation, and when it is unclear which way should be followed. A global problem formulation should therefore describe the current situation (which includes the decision maker), but also objectives and constraints - all in terms of objects, attribute and actions. 
To summarize this section we can state that our interpretation of the conceptualization activity in the problem-solving process comes down to the following three steps:

1. Describe the problem situation in terms of object classes. This step consists of the four activities we mentioned earlier. It requires all kinds of choices to be made concerning the relevance of particular classes, attributes, or actions. When making these choices one should balance between completeness and complexity. "When in doubt, leave it out" is quite an appropriate rule here, since specification and correspondence checks will call for re-conceptualization when that is really necessary.

2. Determine for each object class and/or attribute whether it belongs to the IS, the RS, or the environment of the problem situation. The demarcation rule should be observed at all times.

3. Give a global formulation of the problem using only the "vocabulary" of object classes and their attributes and actions. The object class definitions and this global problem formulation together constitute the conceptual model.

We defer the issue of supporting problem conceptualization to Chapter 4 . In the next section we shall indicate how the transition is made from a conceptual model to an empirical model.

\subsection{PROBLEM SPECIFICATION}

Problem specification essentially is no more than a translation of the conceptual model. The conceptual model provides the decision maker with "building blocks" for the empirical model. The actual construction, however, is not without difficulties. An empirical model must in the first place show a high degree of correspondence with reality. If satisfactory correspondence is not achieved, chances are that a proper solution to the problem cannot be found. Secondly, an empirical model must be suited for experimentation. It has to be formal enough to be programmed into a computer, and it should be possible to use the resulting computer program (recall that we distinguish between computer program and problem-solving programme) as a model within which alternative problem-solving programmes can be tested and evaluated.

Similar to the previous section, we identify the major acivities that are performed during problem specification.
1. Refinement and/or re-definition of object classes: In theory, an empirical model consists only of instances of the object classes defined by the conceptual model. However, in some cases a conceptual model may contain more information than is strictly necessary for the construction of an empirical model. Object classes can be refined by rephrasing their action methods, as we showed in our break down example on page 33. We speak of redefining an object class if, possibly as a result of refining some other object class, an entirely different level of aggregation is required. It may for example be unnecessary to represent each van, each parcel, and each destination as separate objects. Instead, one could use "artificial" object classes like CONVOY, SHIPMENT, and DISTRICT to model groups of vans, parcels, and destinations, respectively. Although the one-to-one mapping of "individual" objects in the RS, like for example "the van with licence plate number FR-08-ER", onto objects in the empirical model is lost when such "artifacts" are used, this model can still show good correspondence, only at a higher level of aggregation.

2. Construction of the empirical model: Once all the necessary object classes have been identified and specified, an empirical model can be constructed by determining the appropriate number of instances for each object class and appropriate values for their attributes, and subsequently instantiating and initializing these objects.

3. Validation of the empirical model: This is the correspondence check in Figure 3-1. Structural validation (comparing at a detailed level the actions of object instances in the model with the actual behavior of the corresponding objects in reality) is possible only in frequently recurring problem situations. Replicative validation (comparing the values of certain attributes of the model with historical or actual data) is another method. Note that validation of the empirical model is usually possible even with ad hoc problems because it is the problem situation, not the problem, that is modeled.

Our research has been focussed mainly on the representation of problem-solving programmes, and on means to support experimenting with different programmes during the solution finding activity. In the next section we present a method for the specification and analysis of programmes. The support aspect will be covered in Chapter 4.

\subsection{TASK ANALYSIS}

In Chapter 2 we stated that problem-solving programmes can be described in terms of tasks and decisions. In this section we present a method for the 
formulation and analysis of programmes, which we shall refer to as "task analysis". To avoid confusion we point out that this phrase has been used earlier by for example Payne (1976) and Lochovsky (1983), but that our method does not build on theirs, but rather on the following ideas and techniques:

\section{Precedence analysis}

In section 2.3 we pointed out that decisions are information processes. Precedence analysis (Langefors 1963) was developed to study certain propertie of information processes. It involves the construction of a so-called "precedence graph", similar to the one depicted in Figure 3-2. In this particular representation the parallelograms denote information sets, and the dots correspond to processes. The edges indicate which information sets are input to a process, and which are output.

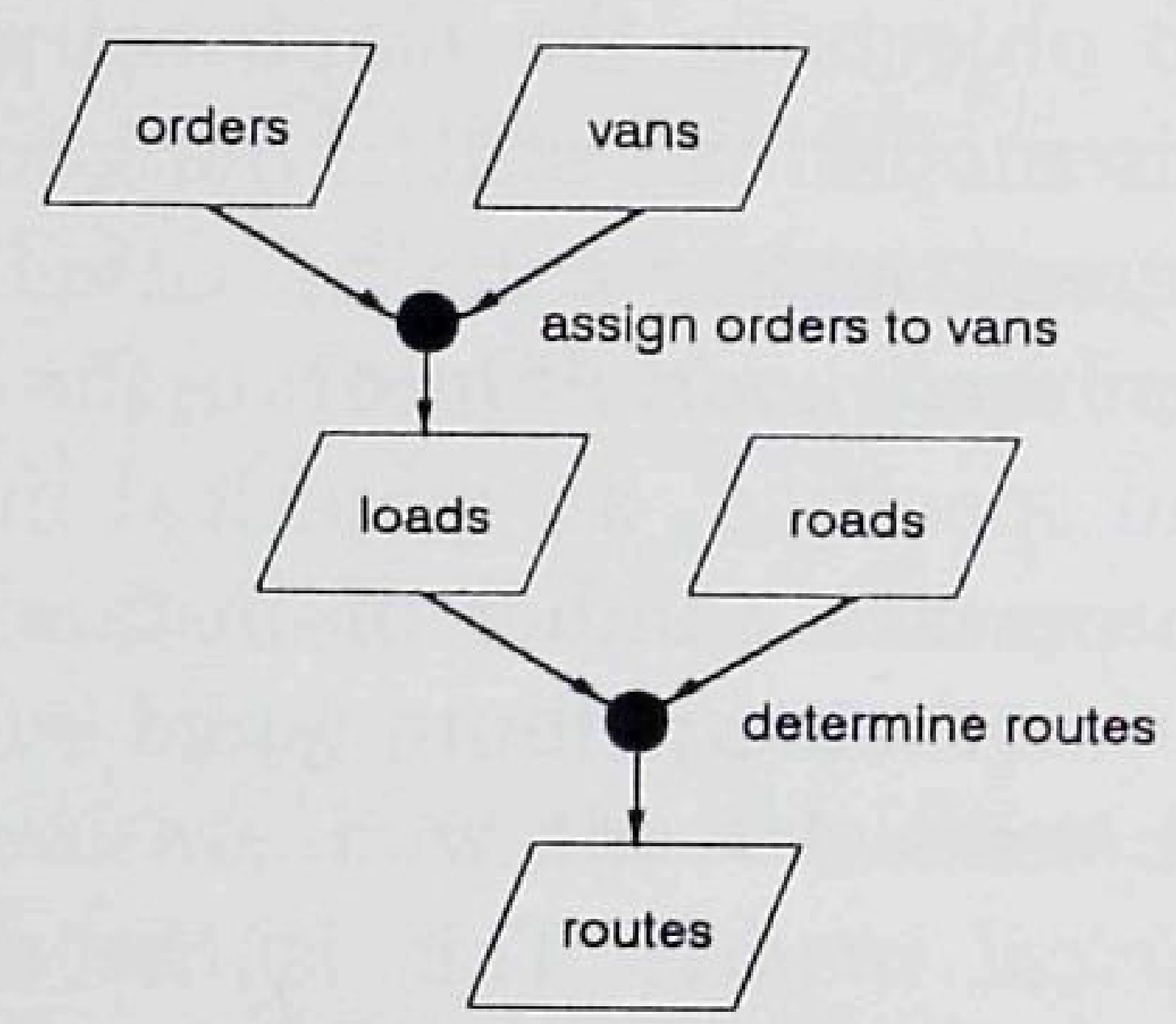

Fgure 3-2. A simple precedence graph for route planning

Analysis of a precedence graph may yield useful information on the sequence in which processes should be performed to obtain the desired final output. Precedence analysis is an element of various information systems design and software engineering methodologies (Lundeberg 1977; DeMarco 1978; Yourdon and Constantine 1979).

We view precedence analysis as an important aid in the detection of coordination problems. If we identify distinct decisions in a problem-solving process, and if we indicate for each decision which part of the problem situation affects the outcome of this decision and which part is affected when this decision is made, it should be possible to apply precedence analysis in order to determine how decisions depend on each other.

\section{Flowcharts}

A flowchart is perhaps the oldest means to visualize the sequence of specific steps in a process or algorithm (Knuth 1963). We therefore assume reader is familiar with their syntax and semantics. For an extensive period flowcharts have been the diagrams to represent programs for computers with a Von Neumann architecture. But the concept of structured - "GOTO-less" - programming (Dijkstra 1968) has led to new diagramming techniques (Nassi and Schneiderman 1972; Jackson 1975). Moreover, flowcharts are not suited for nonprocedural languages, such as LISP and Prolog.

Today, flowcharts are no longer recommended as software engineering aids, but they still are very popular as a means to represent process models. Their major advantage is the ease with which they are read, a property we find absolutely necessary for programme specifications. A reason to consider flowcharts as a means for representing tasks is that flowcharts explicitly represent decisions: the characteristic "diamonds" that indicate conditional branches.

\section{Petri nets}

Since tasks may be performed by multiple actors, we must allow for concurrency in task descriptions. Most of the methods for the explicit representation of concurrency that we found in information systems literature are based on the "Petri net"-concept (Petri 1962). Variations on Petri nets are widely used to describe problem situations that involve coordination (Holt and Cashman 1981; Richter et al. 1987; Holt 1988). Indicative of its growing popularity as an instrument in information systems design is the fact that most of the contributions to the IFIP WG8.4 conference on the design process of office information systems (Pernici and Verrijn-Stuart 1989) relate to some extension of Petri nets.

A Petri net is a connected, directed graph. A node in a Petri net is either a "place" (represented graphically as a circle) or a "transition" (represented as a bar or rectangle). The places from which an edge leading to some transition $t$ originates are called the "input places" of $t$; the places at the end of the edges leaving $t$ are called the "output places" of $t$. Edges between nodes of the same type are not allowed.

Places are usually associated with states or substates of the modeled system, and transitions with activities. Input places of a transition model the conditions that have to be met before that transition can "fire", that is to say, before the activities it represents can take place. Its output places model the conditions that are fulfilled the moment these activities have been performed.

The system's dynamic properties can be modeled by putting "tokens" into a number of places in the net. The way tokens "move around" in the net is determined by the firing rules. Different types of Petri nets may have different firing rules. The basic rule is that a transition will only fire when there is at least one token in each of its input places. When a transition fires, one token is removed from each of its input places, and one token is added to each output place. Thus, tokens are generated if a transition fires that has more output places than input places, or are consumed if the number of input places exceeds that of the output places. 
Using only the basic firing rule, we can model the loading of a delivery van as the Petri net shown in Figure 3-3. Transition $t_{1}$ models the arrival of a parcel on the conveyor belt. By placing a token in $p_{1}$ we would initiate a continuous stream of parcels. Transition $t_{2}$ models the activity of taking the parcel from the belt and storing it in the van. The number of tokens placed in $p_{2}$ corresponds with the van's capacity.

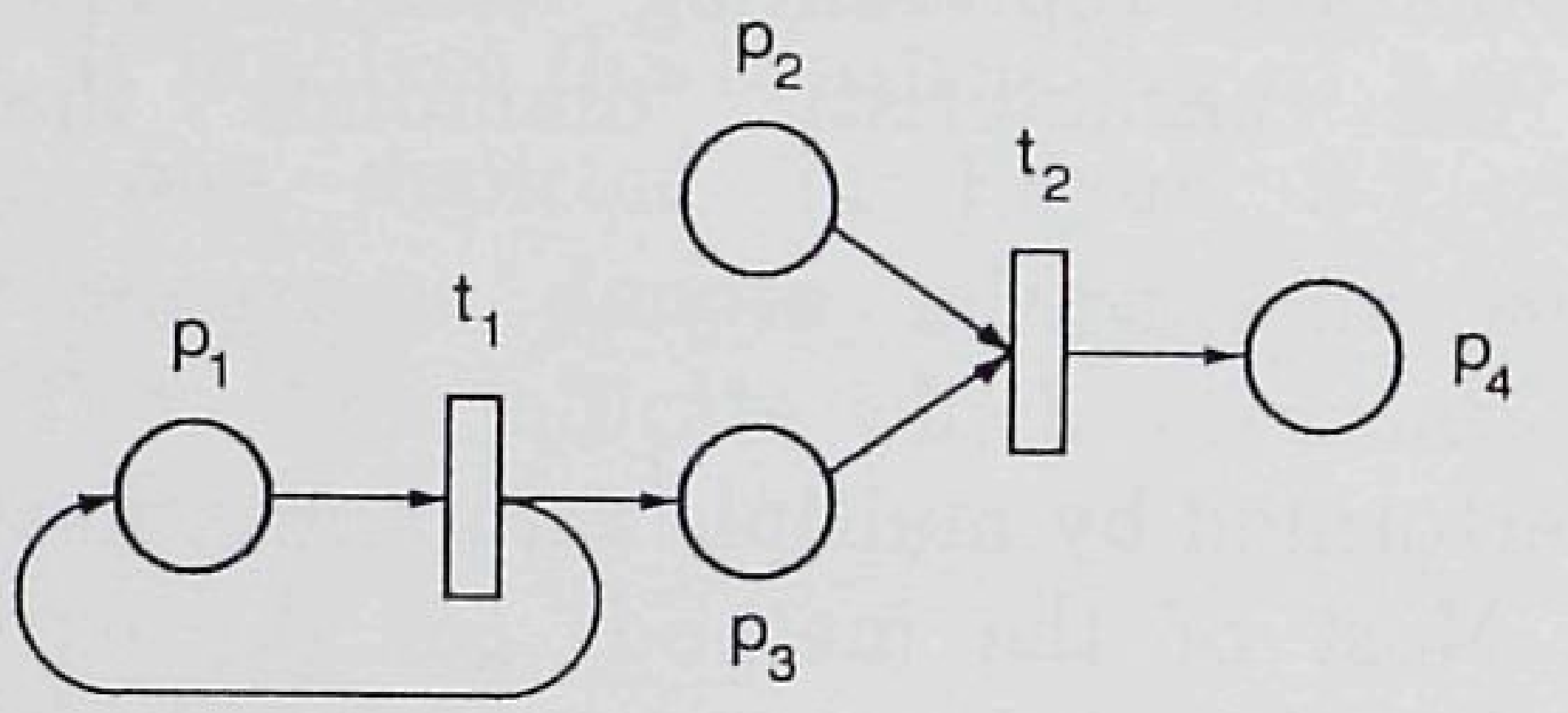

Fgure 33. A Petri net representation of loading a delivery van

If we wish to model the delivery of a parcel as well, we need a more complex net. We also need some means to model the fact that loading parcels and being on route delivering them are two separate phases, and that a van will not depart before it is fully loaded. We therefore added a so-called "weight function" to the net. By labeling the edges $\left(p_{2}, t_{2}\right)$ and $\left(p_{4}, t_{4}\right)$ of the Petri net in Figure 3-4 with the van's capacity $c$, we indicate that the transitions $t_{2}$ and $t_{4}$ cannot fire unless there are at least $c$ tokens in their input place. By labeling their outgoing edges $\left(t_{2}, p_{3}\right)$ and $\left(t_{4}, p_{5}\right)$ we indicate that when they fire, $c$ tokens are added to their output place.

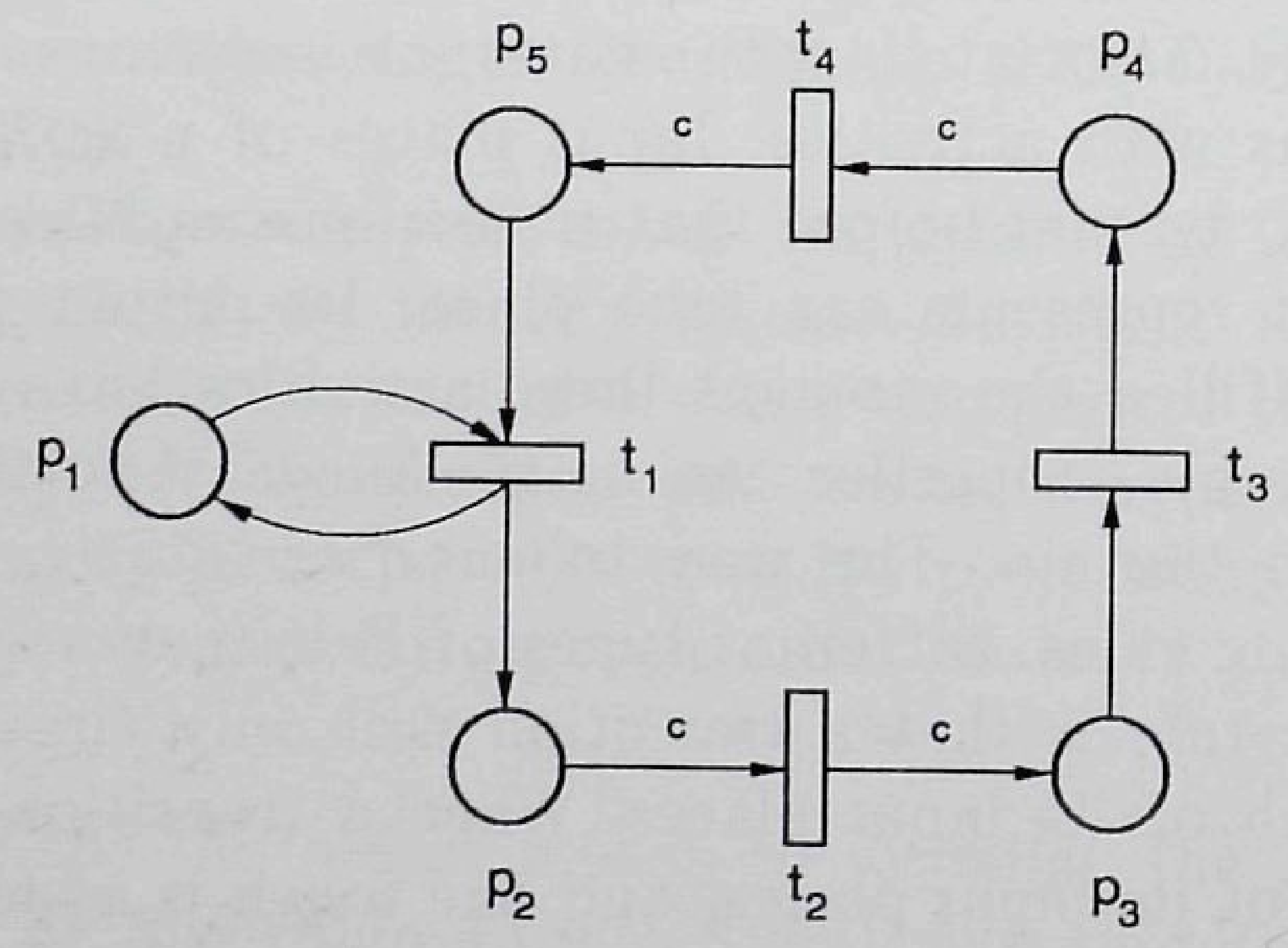

Fgure 3-4. An extended Petri net representation of a delivery van
To model the loading and delivery process we add some tokens to the net. By putting a token in $p_{1}$ we indicate that there are parcels waiting. To indicate that an empty van has arrived we put $c$ tokens in $p_{5}$. Transition $t_{1}$ models one parcel being loaded. $t_{1}$ must fire $c$ times before the transition $t_{2}$ can fire. When it transition $t_{3}$, modeling the delivery of a parcel, must fire $c$ times, as there have transition $t_{3}$, modeling the delivery of a parcel, must return of the empty van. Adding a weight function to the edges of a Petri net is but one of many

ys in which their expressive power can be enhanced. To model the behavior ways in which their expressive power can be enhanced. Th the semantics of Petri of information systems, Kung and S $\phi$ lvberg (1986) extend the semantics of Petr nets by relating places to entity classes, and by add (1988) combines Petri net transitions as preconditions and postconditions. Lee (1988) combines Petri nets with a form of logic to describe bureaucratic procedures.

But the Petri net concept has some severe drawbacks. When Petri nets ar used to model larger systems, or when they have to specify rules that are more complex than those in Figure 3-3 and 3-4, the results soon become unreadable. Even when augmented with preconditions and postconditions specified in some logic, a Petri net does not represent the flow of events as clearly as a flowchart does. We feel that when it comes to specifying programmes, readability does. We feel that when it comes to specifying programmes, concurrent aspects.

We emphasize that our method of task analysis is not a Petri net derivative. The precedence-graph and flowchart aspects have dominated from the start. The Petri net-like properties did not become apparent until we had defined the dynamic behavior of programmes.

Task analysis results in a concise formulation of a problem-solving programme. During task analysis, two representations of this programme are used: a "decision structure". The decision structure specifies the "decision structure" and a "task structure". The decision structure specifies the essential decisions that may have to be made in the problem-solving process. The task structure addresses the coordination problem, since it specifies how a
task is divided into subtasks, and also the order in which the decisions have to be made.

In the following paragraphs we define the syntax and a semantics of decision structures and task structures. By "syntax" we mean their "building blocks", and the rules that govern the way in which these elements may be put together. Thus, the syntax of Petri nets would consist of places, transitions and tokens, the rule that a net should be a connected, directed and bipartitioned graph, and the rule that only places can be marked by putting tokens in them. "Semantics" refers to the meaning of syntactically correct constructs, or more specifically, their "dynamic interpretation". Essentially this is the set of rules 
that defines the behavior of such constructs. For Petri nets this set consists of the rules concerning the marking of places and the firing of transitions.

Decision structures

First, we consider the syntax of decision structures. The "building blocks" are "decision" and "information". In the context of task analysis we use a rather narrow definition of information:

\section{Definition 3.1}

Information corresponds to a collection of one or more attributes of object instances that are part of the empirical model.

Information is graphically represented as simple rectangles, such as the one in Figure 3-5(a). Each rectangle contains a brief description of the information it represents.

\section{Definition 3.2}

A decision is a 3 -tuple $d=\left(I_{d}, A_{d}, S_{d}\right)$, where $I_{d}$ denotes the information that "influences" $d$, and $A_{d}$ the information that "i affected by" $d$, while $S_{d}$ stands for the "support component" of $d$.

Information influences a decision when it affects the making - and therefore the outcome - of that decision. Information is affected by a decision if making that decision may result in changes in the values of attributes to which the information corresponds. We say "may result" because the outcome of $d$ could be not to change any attribute values (consider for example the decision not to add an extra destination to a route plan). The support component of a decision is the collection of means that are explicitly meant to facilitate the making and/or implementation of that decision. For example, means to retrieve, process, and present data to the decision maker, or means to communicate the choices that have been made. Thus, the support component of a decision is not relevant during task analysis itself, but serves as the "link" between decision and information technology.

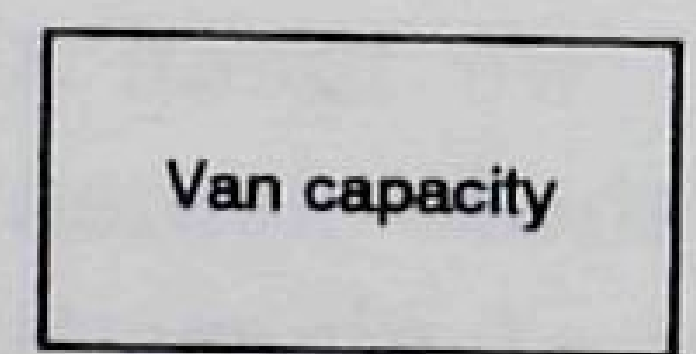

(a) information

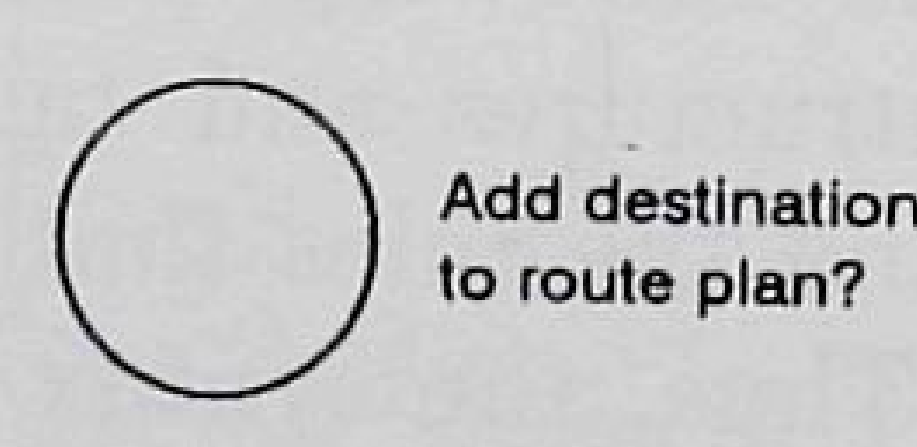

(b) decision
Foure 35. Information and decision symbols
A decision is graphically represented as a circle, as the one in Figure 3-5(b). A brief description is always displayed right next to it. The descriptions of both information and decisions have to be unique in the sense that they are identifiers. For example, the rectangles labeled "Info3" and "Info5" occur twice in Figure 3-6(a), but they refer to the same attributes and/or object instances. This accounts for the edges $\left(d_{3}, d_{2}\right)$ and $\left(d_{4}, d_{3}\right)$ in Figure $3-6(\mathrm{~b})$.

\section{Definition 3.3}

The decision structure $\mathcal{D}_{\mathrm{P}}$ of a problem $\mathbf{P}$ is a directed graph $(V, E)$, in which $V=\left\{d_{1}, \ldots, d_{\mathrm{n}}\right\}$ is the collection of all decisions that can be identified when analyzing $\mathrm{P}$, and $E=\left\{\left(d_{\mathrm{i}}, d_{\mathrm{j}}\right) \mid A_{d_{\mathrm{i}}} \cap I_{d_{\mathrm{j}}} \neq \varnothing\right\}$.

In other words, $E$ is the subset of $V \times V$ that includes an edge $e_{\mathrm{ij}}$ leading from decision $d_{\mathrm{i}}$ to decision $d_{\mathrm{j}}$ in $\mathcal{D}_{\mathrm{P}}$ if and only if the affected part of $d_{\mathrm{i}}$ overlaps with the influencing part of $d_{\mathrm{j}}$.

A decision structure can be shown with or without information. In an "expanded" decision structure the relation between decisions and information is shown. In a "condensed" decision structure the influencing and affected information is not shown, but the dependencies between decisions are still visible. Both representations are shown in Figure 3-6. In general, the support component of a decision is not visualized in decision structures, as it is irrelevant in task analysis. It is important only when the interaction between different support objects is specified and analyzed, an issue we defer to Chapter 4.

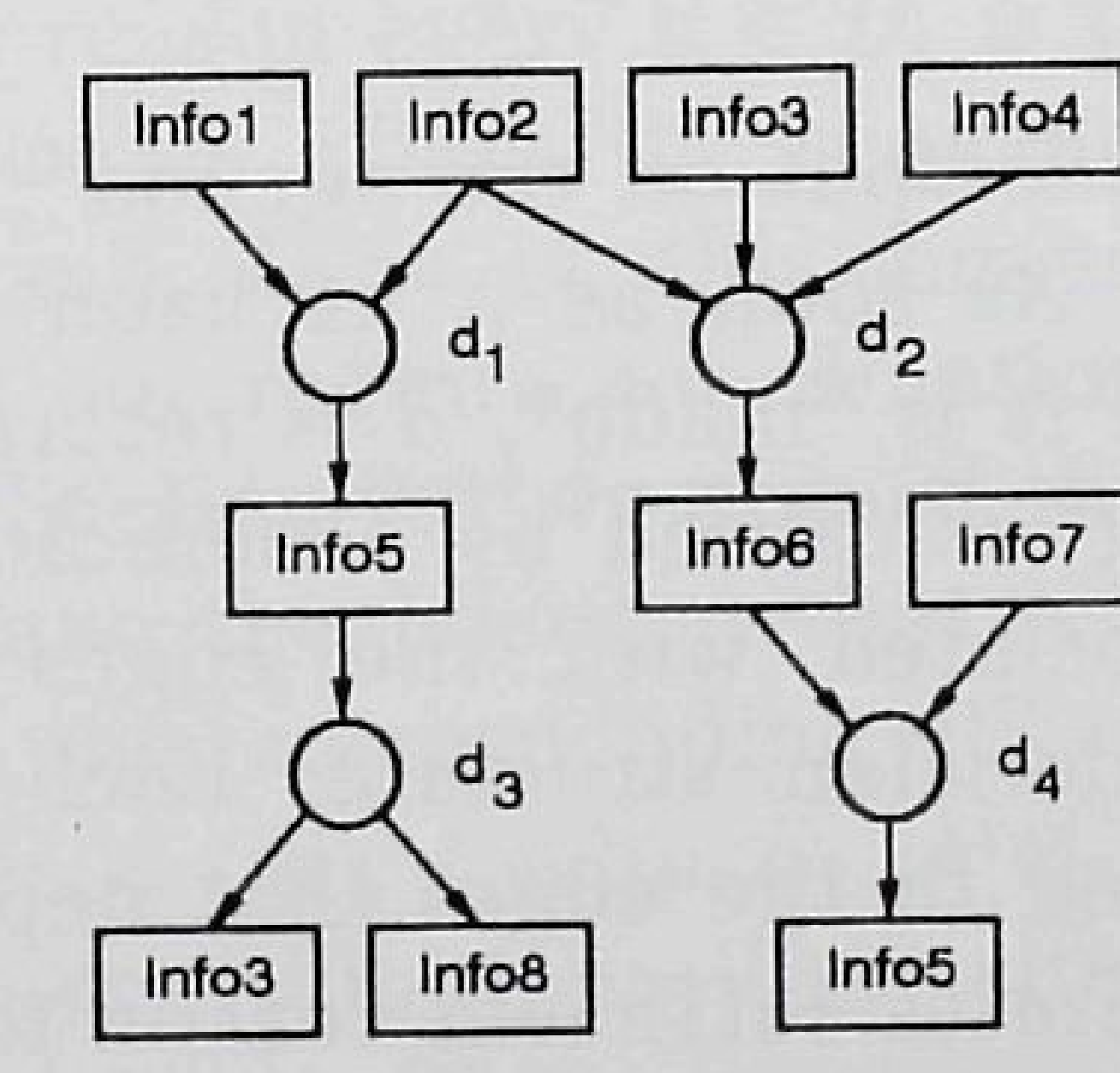

(a) expanded

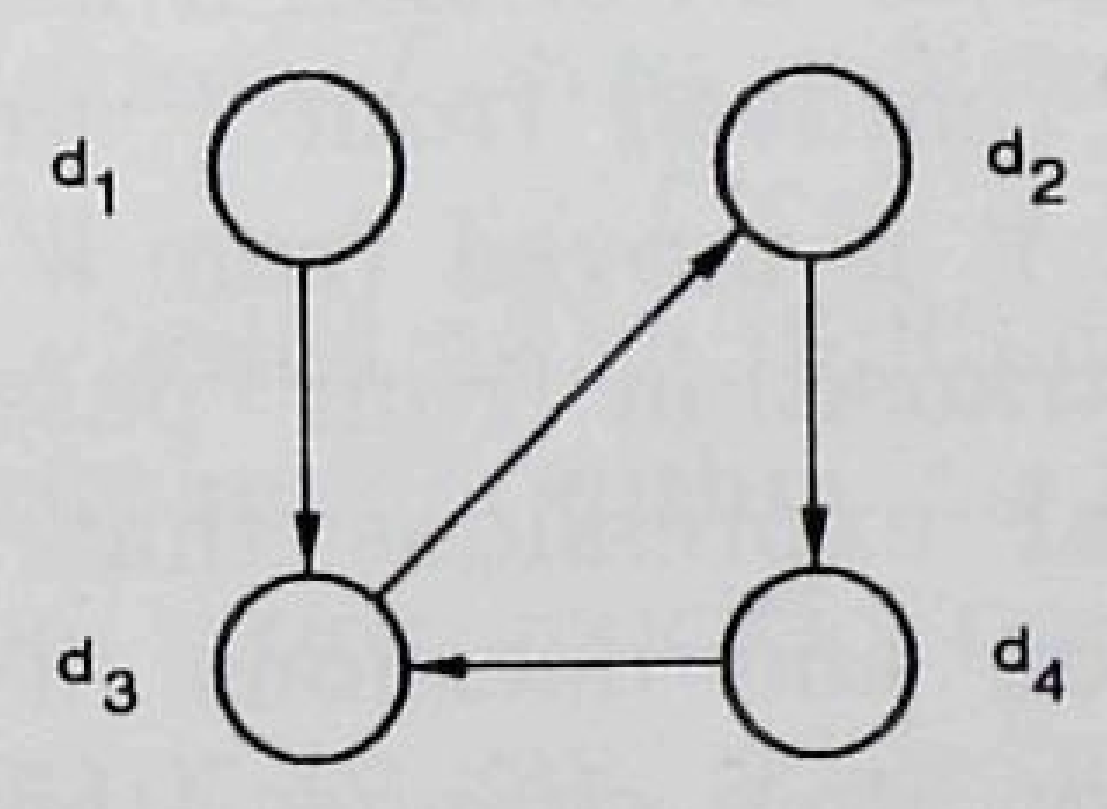

(b) condensed
Figure 3-6. Two graphical representations of one decision structure

From its definition one can conclude that a decision structure essentially is a precedence graph. Such a graph in itself does not express any dynamics other than that information flows in the direction of the directed edges, but this can reveal a treasure of information concerning the sequencing of decisions. As a 
directed graph, a decision structure can also be analyzed using techniques from graph theory, such as the identification of cycles and (strongly) connected components. If a decision structure is not a connected graph, this may indicate omissions in the conceptual or empirical model, or - if the analysis shows no gaps two or more completely unrelated subproblems. Cycles and strongly connected components clearly indicate a need for coordination.

We wish to extend the semantics of a decision structure in such a way that the procedural nature of the decision process can be analyzed in more detail. For this purpose we borrow two concepts from the literature on artificial intelligence: "agenda" and "demon" (Barr and Feigenbaum 1981). Basically, the agenda of a decision structure is a dynamic list of decisions that at any moment indicates which decisions are to be made at that moment:

\section{Definition 3.4}

The agenda $\mathcal{A}_{D}$ associated with a decision structure $\mathcal{D}_{\mathrm{P}}=(V, E)$ of a problem $\mathbf{P}$ is a 3 -tuple $(A, W, P)$, where $A, W$, and $P$ are disjunct subsets of $V$ such that $A \cup W \cup P=V$.

The elements of subset $A$ are the decisions that are "active". Subset $W$ contains those that are "waiting", and $P$ those that are "passive". A decision $d$ is said to be active when some decision maker is in the process of examining it influencing part $I_{d}$, deciding, and possibly changing the affected part $A_{d}$. A decision is waiting when it should be made, but when no decision maker is available. A decision is passive if its influencing part provides no reason why it should be made.

Whenever a decision $d$ has to be made, it is "put on the agenda", meaning that $d$ is removed from $P$ and added to $W$. As soon as a decision maker available, it is moved from $W$ to $A$ and

The transition from "passive" to "waiting" is caused by a demon. Demon are logical expressions that should be associated with the edges between information and decisions in an expanded decision information item changes, the demons attached to the edges that depart from this item determine whether this change constitutes sufficient reason to put the decision at the other end of the edge on the agenda. In other words, by attaching appropriate demons to edges one can specify under which circumstances a specific decision has to be made.

Note that in a decision structure decisions have no memory whatsoever They do not store information concerning previous choices, nor do they hold any decision rules. Memory and decision rules belong to the decision make (they might later become part of the support component, but that is another matter altogether).
Similarly, the information items represented by rectangles are but references to objects and attributes. They do not hold information themselves. By making decision $d$ the decision maker does not change the contents of the "boxes" in $A_{d}$ directly. Rather, his choice affects a number of objects in reality or in the empirical model, and this becomes visible as changes in the values of (some of) the attributes in $A_{d}$.

Finally we wish to point out that the value of certain attributes in $I_{d}$ and even $A_{d}$ may change without decision $d$ or any other decision in $V$ being made. These attributes will belong to objects that are part of the IS/RS-combination's environment.

\section{Task structures}

In section 2.3 we described the relation between problem, decision, and task. We defined a task as a problem-solving process. A decision was seen as the choice phase of that problem-solving process. Also, we found that problems are recursive in the sense that solving a problem generates subproblems. Building on these ideas, we formally define a task as follows:

\section{Definition 3.5}

A task is an ordered pair $t=\left(D_{t}, T_{t}\right)$, where $D_{t}$ is a set of decisions, and $T_{t}$ a set of tasks called the "subtasks" of $t$.

If $T_{t} \neq \varnothing$ this means that performing task $t$ requires the performance of one or more subtasks, possibly more than once. If performing task $t$ involves some kind of interaction between the subtasks in $T_{t}$, these subtasks have to be coordinated. For this reason every $d \in D_{t}$ is then called a "coordinating decision". Coordinating decisions affect the sequence in which subtasks are performed. If, on the other hand, $D_{t}=\varnothing$, this means that the subtasks in $T_{t}$ are independent tasks that do not require coordination.

In Section 2.4 we argued that a task can be viewed as an IS/RS-combination if we take the position that a decision is always made within an IS, while it affects the corresponding RS. We can now be more specific on this issue. Consider a task $t$ with $D_{t} \neq \varnothing$ and $T_{t} \neq \varnothing$, that is to say, with subtasks that require coordination. This situation corresponds to the "direct supervision" coordinating mechanism, depicted by Figure 2-1 on page 23. The higher level IS (the IS-component of task $t$ ) coordinates the lower level IS/RS-combinations (the subtasks in $T_{t}$ ) through a decision process that consists of the decisions in $D_{t}$.

At the lowest level, where no subtasks are identified $\left(T_{t}=\varnothing\right)$, coordination is not required. In this case $t$ is called a "leaf task", and the decisions in $D_{t}$ are referred to as "leaf decisions". Leaf decisions do not relate to the coordination problem (they do not determine which subtask has to be performed), but to the actual problem at hand. In our example on page 24 , the decisions made by project manager Pete are coordinating decisions that affect the tasks of the 
analysts Huey and Dean. Dean's decision to change the PID format is a leaf decision in one of his subtasks, but his decision to inform Huey about this change is a coordinating decision that may affect the way in which Huey performs one of his subtasks.

In Chapter 2 we called a task "structured" if a problem-solving programme for that task exists, and "ill-structured" otherwise. When graphically representing tasks, we also distinguish between structured and ill-structured tasks. As is shown in Figure 3-7, task symbols are characterized by their double rim. A structured task is represented by a rectangle that is somewhat larger than the informationrectangle, while an ill-structured task is displayed as a "soft" rectangle (Olle et al. 1988, page 199).

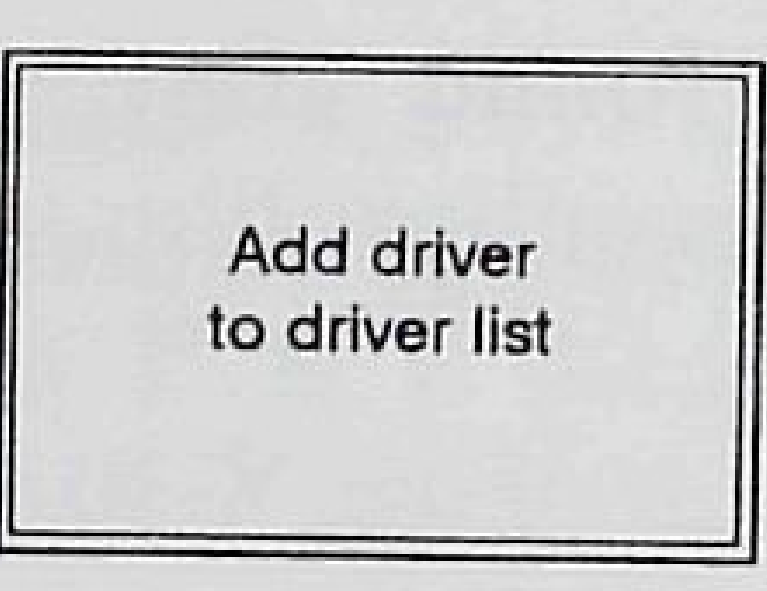

(a) structured

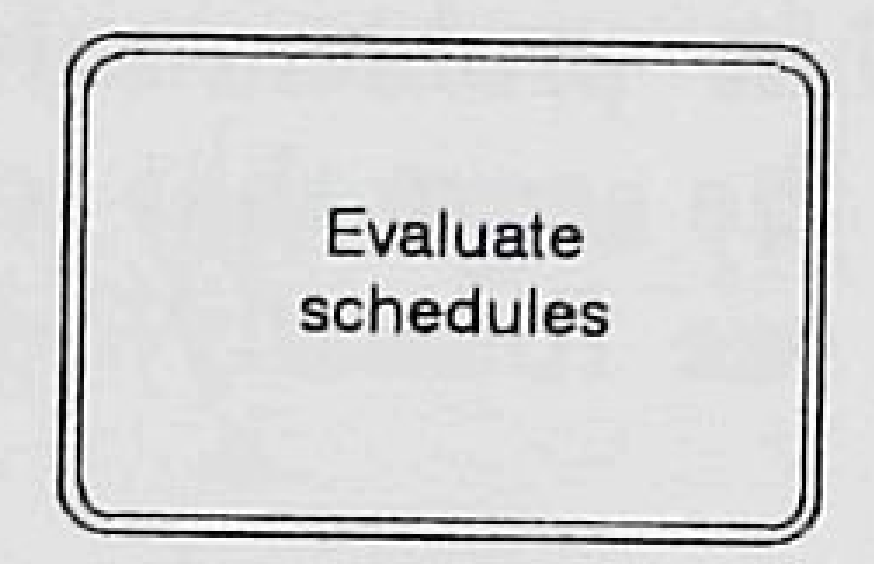

(b) ill-structured
Figure 3-7. Task symbols

By defining tasks recursively in terms of subtasks, a hierarchical structure as depicted in Figure 3-8(a) is created. It is tempting to consider this hierarchy as the task structure of a problem, an idea that is advocated by for example Lochovsky (1983) and Croft and Lefkowitz (1984). However, such a hierarchy lacks the procedural aspect. Coordinating decisions make it possible to express this procedural aspect of problem solving. Where the recursive task-subtask relation creates an hierarchical structure, the task-decision relation facilitates the making of a flowchart-like structure as is shown in Figure $3-8(\mathrm{~b})$. We combine both aspects in the following definition:

\section{Definition 3.6}

The task structure $\mathcal{J}_{\mathrm{P}}$ of a problem $\mathbf{P}$ is an ordered pair $\left(\mathcal{H}_{\mathrm{P}}, \mathcal{F}_{\mathrm{P}}\right)$ where $\mathcal{H}_{\mathrm{P}}$ denotes the "task hierarch" and $\mathcal{F}_{\mathrm{P}}$ stands for the "flow relation" between all coordinating decisions in $\mathcal{T}_{\mathrm{p}}$.

The task hierarchy is identical to what Lochovsky (1983) calls "task structure" A task hierarchy need not be a tree, since a task may be a subtask of more than one other task. The task of looking up a particular order in the order list could be a subtask of both "Select unprocessed order" and "Plan" in Figure $3-8$.

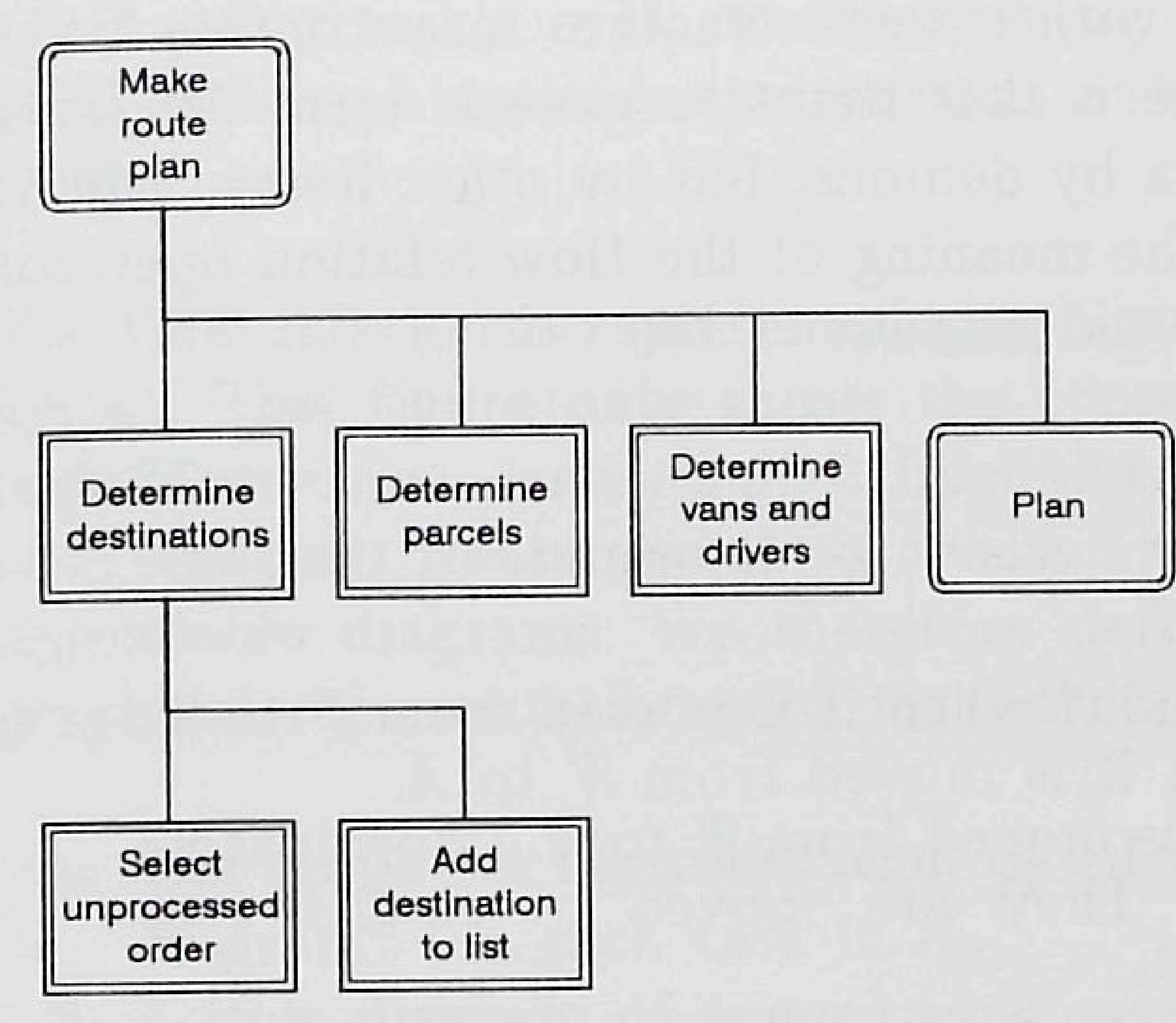

(a) as a hierarchy

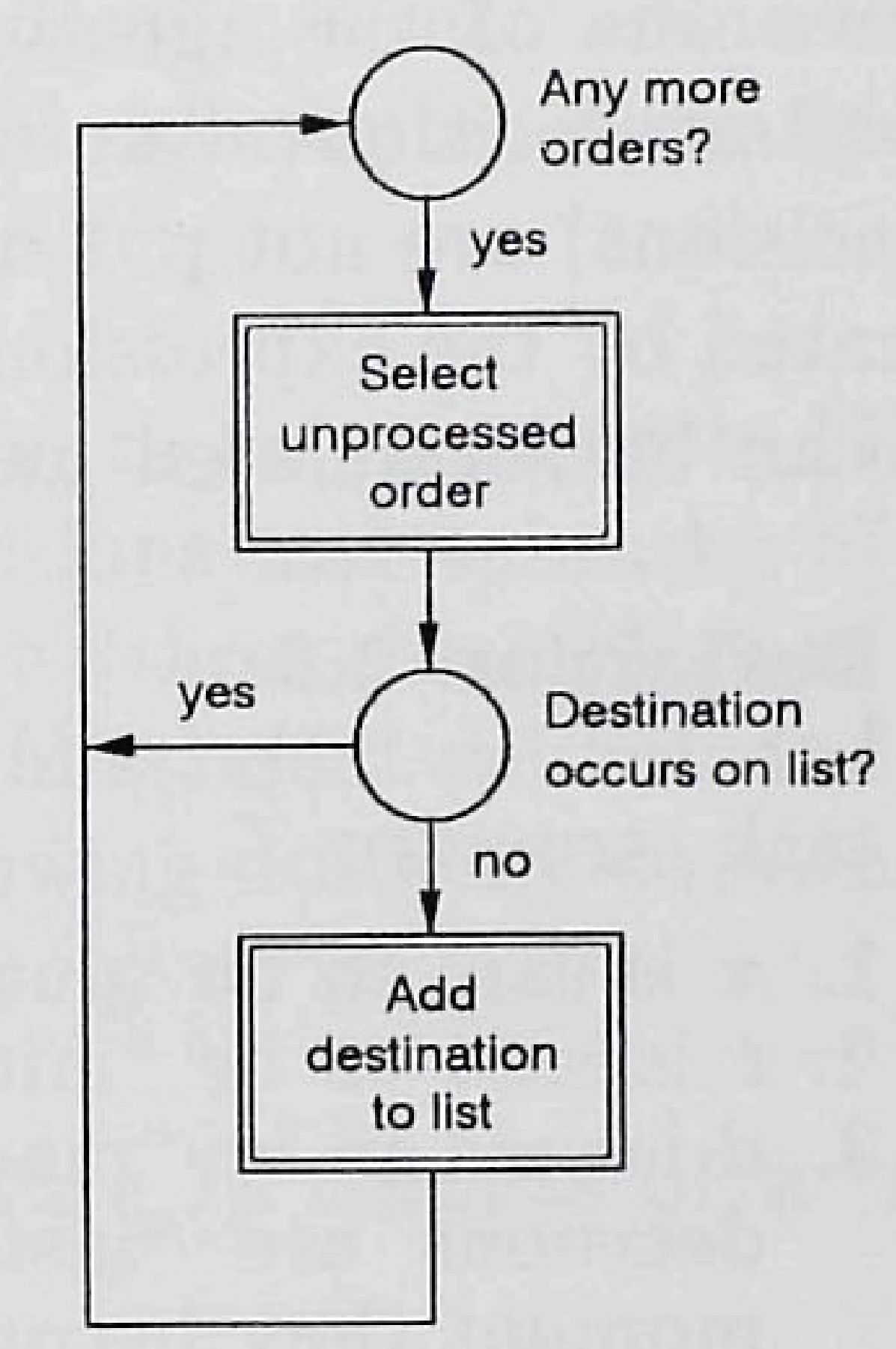

(b) as a flowchart
Flgure 3-8. Two different views on task structure

However, a task hierarchy may not contain cycles: If $t_{2}$ is a subtask of $t_{1}, t_{1}$ cannot be a subtask of $t_{2}$ or any of its descendants. Consequently, $\mathcal{H}_{\mathrm{P}}$ is formally defined by:

\section{Definition 3.7}

A task hierarchy $\mathcal{H}_{\mathrm{P}}$ for a problem $\mathbf{P}$ is a directed graph $(V, E)$, in which $V=\left\{t_{1}, \ldots, t_{n}\right\}$ is the collection of all tasks that have to be performed when solving $\mathbf{P}$, and $E=\left\{\left(t_{\mathrm{i}}, t_{\mathrm{j}}\right) \mid t_{\mathrm{j}} \in T_{t_{\mathrm{i}}}\right\}$ such that $E$ constitutes a partial ordering of $V$.

The flow relation $\mathcal{F}_{\mathrm{p}}$ plays an important role in the dynamic interpretation of a task structure. It is defined in terms of an agenda that is somewhat different from the agenda associated with a decision structure.

\section{Definition 3.8}

Let $\mathcal{T}_{\mathrm{P}}$ be a task structure with task hierarchy $\mathcal{H}_{\mathrm{P}}=(V, E)$, and let $C$ be the set of all coordinating decisions of all tasks in $V$, defined by:

$$
C=\bigcup_{\substack{t \in V \\ T_{t} \neq \varnothing}} D_{t}
$$

The agenda $\mathcal{A}_{T}$ associated with $\mathcal{T}_{\mathrm{P}}$ is a 3 -tuple $(A, W, P)$ in which $A$, $W$ and $P$ are disjunct subsets of $V \cup C$ such that $A \cup W \cup P=V \cup C$, while the flow relation of $\mathcal{J}_{\mathrm{P}}$ is defined by:

$$
\mathcal{F}_{\mathrm{P}}=\{(x, y, \lambda) \mid x \in V \cup C \wedge y \in V \cup C \wedge x \stackrel{\lambda}{\rightarrow} y\}
$$


A first difference with $\mathcal{A}_{D}$ in Definition 3.4 is that the Active, $W$ aiting, and $P$ assive sets of the agenda associated with a task structure may contain tasks as well as decisions. Another difference is that items (a generic term for tasks or decisions) are not put on the agenda by demons, but by other items. This is indicated by the expression " $x \stackrel{\lambda}{\rightarrow} y$ ". The meaning of the flow relation operator

" $\lambda$," can be formulated more precisely as follows:

\section{Definition 3.9}

Let item $x$, task $t$ and coordinating decision $d$ all be part of the same task structure $\mathcal{T}_{\mathrm{P}}$.

1. $x$ is said to be "put on the agenda" when it is moved from $P$ to $W$

2. $t$ is said to be "initiated" when it is moved from $W$ to $A$

$3 . d$ is said to be "made" when it is moved from $W$ to $A$. Coordinating decisions are "instantaneous": They are moved to $P$ the very moment they become part of $A$.

4. The elements of the $\operatorname{set}^{\bullet} x=\{y \mid y \wedge x\}$ are called the "immediate "precursors" of $x$, while the elements of $x^{\bullet}=\{y \mid x \wedge y\}$ the "immediate successors" of $x$.

5. The moment task $t$ is initiated, its "initial items" $I_{t}$ defined as $\left\{x \mid x \in D_{t} \cup T_{t} \wedge{ }^{\bullet} x=\varnothing\right\}$ are put on the agenda.

6. Let $t \in A,\left(D_{t} \cup T_{t}\right) \cap A=\{x\}$, and let $S \subseteq x^{\bullet}$ be the set of items that in a given situation are put on the agenda when $x$ is moved from $A$ to $P$. Task $t$ is said to "terminate" when $S=\varnothing$, while $t$ is said to "abort" when $S \neq \varnothing$ and $S \cap\left(D_{t} \cup T_{t}\right)=\varnothing$.

7. When a task terminates or aborts, it is moved from $A$ to $P$

8. Every $x \in t^{\bullet}$ is put on the agenda when task $t$ terminates. By contrast, if task $t$ aborts, these items are not put on the agenda.

9. Every item $x \in d^{\bullet}$ is put on the agenda the moment decision $d$ is made if and only if the label $\lambda$ associated with the edge $(d, x)$ evaluates true in the context of the description of $d$.

Labels are not bound by a specific syntax because it may be undesireable or even impossible to specify a coordinating decision or its potential outcomes in terms of some formal logic.

Flows departing from tasks have empty labels, since evaluation takes place only in the context of a coordinating decision. Note that the labels of flows that depart from the same decision need not indicate mutually exclusive conditions. Also, the sequence in which items are added to the agenda is not necessarily the sequence in which they will be made/initiated. If a specific sequence is required this should be realized by defining appropriate flows.

There is one important restriction with respect to the elements of the flow relation: $\mathcal{F}_{\mathrm{P}}$ cannot contain tuples $(x, t)$ if $x \in D_{t_{1}} \cup T_{t_{1}}$ and $t \in T_{t_{2}}$ for some tasks $t_{1}$ and $t_{2}$ with $t_{1} \neq t_{2}$. This restriction directly follows from the information paradigm. Recall that we view a task $t$ as an IS/RS-combination in which, provided that $T_{t} \neq \varnothing$, the RS consists of the subtasks in $T_{t}$, while the coordinating decisions in $D_{t}$ constitute the IS. If some task or decision $x \notin D_{t}$ could put a task $t \in D_{t}$ on the agenda, this would mean a direct influence of the IS/RS-combination's environment on the RS, which violates the demarcation rule.

The flow relation is represented graphically by arrows, as can be seen in Figure $3-8$ (b). This figure only shows the coordinating decisions and subtasks of the task "Determine destinations". Depicting the complete (three-dimensional) task structure of "Route planning" as a two-dimensional graph would result in unreadable diagrams. We therefore define the following rules to construct a graphical representation of a task structure:

1. The graphical representation $\mathcal{G}_{\mathrm{P}}$ of a task structure $\mathcal{J}_{\mathrm{P}}$ consists of a "flow diagram" for each task in $\mathcal{H}_{\mathrm{p}}$.

2. A flow diagram of some task $t$ contains a symbol for each decision in $D_{t}$, and for each subtask in $T_{t}$.

3. Task symbols are characterized by a double rim, as shown in Figure 3-7 on page 44. Decision symbols are circles, usually like the one in Figure 3-9(a).

4. If a flow diagram depicts a task $t$ with $D_{t} \cup T_{t} \neq \varnothing$, the symbol for $t$ must

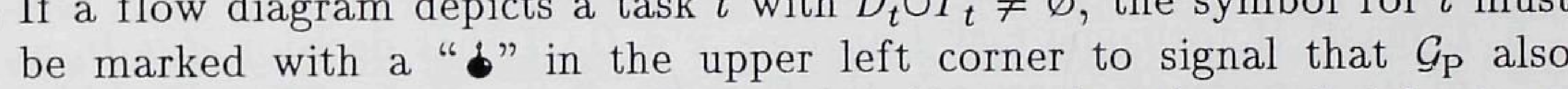
contains a diagram for task $t$ (the bomb indicates that the symbol for $t$ can "explode").

5. If $\mathcal{F}_{\mathrm{P}}$ includes a flow from some item $x \in D_{t_{1}} \cup T_{t_{1}}$ to a decision $d \in D_{t_{2}}$, and $t_{1} \neq t_{2}$, this decision $d$ is also drawn in the flow diagram of $t_{1}$, but as a dotted instead of a full circle, like the one in Figure 3-9(b).

6 . If $\bullet d=\varnothing$, the circle representing decision $d$ is emphasized, as is done in Figure 3-9(c).

7. Each flow $(x, y, \lambda) \in \mathcal{F}_{\mathrm{P}}$ such that $x$ and $y$ are both drawn is depicted by an arrow labeled with $\lambda$ that connects the symbol for $x$ with the symbol for $y$.

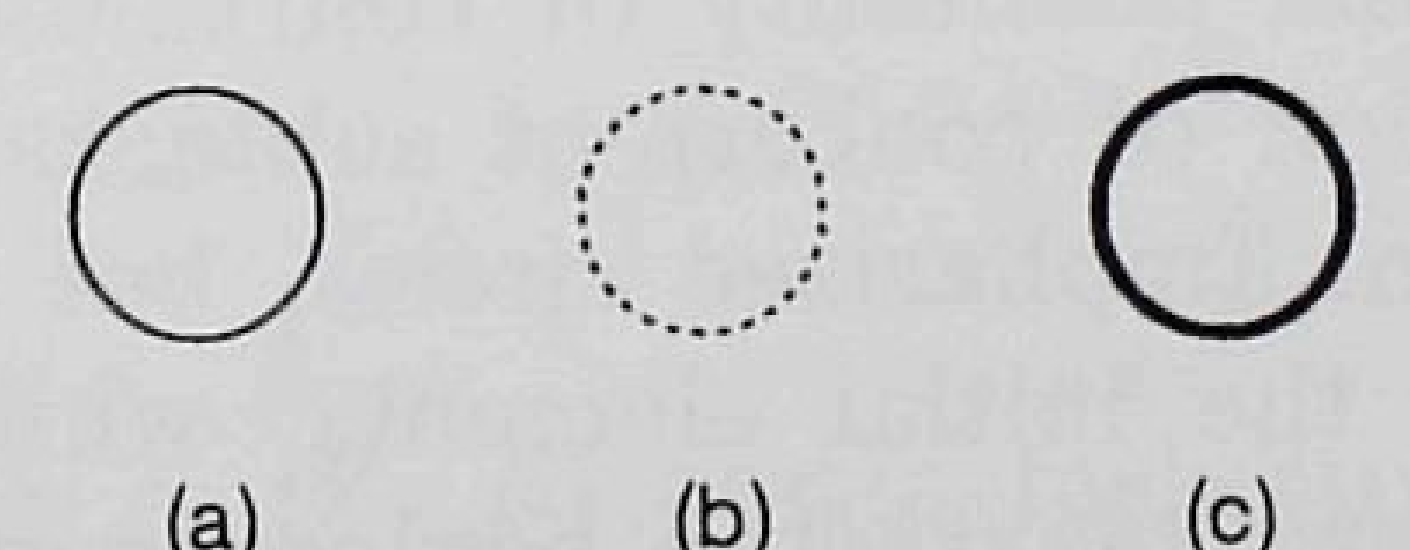

Flgure 3-9. Decision symbols

We now wish to point out a number of properties of task structures that follow directly from our definition of the flow relation. There are five properties that we consider to be of interest: 
1. (Concurrency) If $t$ is a task with initial items $I_{t}=\left\{x_{1}, \ldots, x_{n}\right\}$, or if $x$ is an item with $x^{\bullet}=\left\{x_{1}, \ldots, x_{\mathrm{m}}\right\}$, this introduces concurrency in the sense that the items $x_{\mathrm{i}}$, but also $x_{\mathrm{i}}^{\bullet}$ and so forth, may be active simultaneously. When it is possible for two items $x$ and $y$ to we write $x \| y$. Note that a coordinating dec only when the conditions associated with the flows from $d$ to the items $x \in d^{\bullet}$ are not mutually exclusive. If, for example, two condition associated with different flows from $d$ are satisfied when $d$ is made, both items at the end of the flows will be put on the agenda.

2. (Sequencing) By defining tasks $t_{1}, \ldots, t_{\mathrm{n}}$ such that ${ }^{\bullet} t_{\mathrm{i}}=\left\{t_{\mathrm{i}-1}\right\}$ for $\mathrm{i}=2, \ldots, \mathrm{n}$, these tasks will be performed strictly sequential.

3. (Synchronization) By defining $t_{1}, \ldots, t_{\mathrm{n}}$ as subtasks of a task $t$, any item $x$ with $x=\{t\}$ will not be put on the agenda until each task $t_{\mathrm{i}}$ has terminated.

4. (Confusion) If $\left\{t_{\mathrm{i}}, t_{\mathrm{j}}\right\} \subseteq{ }^{\bullet} t$ and $t_{\mathrm{i}} \| t_{\mathrm{j}}$, it is possible that $t_{\mathrm{i}}$ terminates before $t_{\mathrm{j}}$. When $t_{\mathrm{i}}$ terminates, $t$ is initiated. If $t_{\mathrm{j}}$ terminates while $t$ is still active, we speak of "confusion", since $t_{\mathrm{j}}$ will also initiate $t$, which may have consequences for the way $t$ is performed.

5. (Signal) If for some task $t$ an item $x \in D_{t} \cup T_{t}$ has decision $d \notin D_{t}$ as one of its immediate successors, the flow $(x, d)$ is called a "signal". A signal is a coordinating mechanism to model emergencies - situations that call for specific problem-solving activities in areas that are not "covered" by the subtasks of $t$. The outcome of $d$ will determine which action (if any) should be taken within the task(s) of which $d$ is a coordinating decision.

The graphical representation rules make sure that these properties are visualized in flow diagrams. Multiple arrows departing from a task symbol immediately point out concurrency. This also holds for disconnected compon immediately diagram, as long as they do not start with an unemphasized circle (such decisions are no initial items). Sequencing appears quite naturally as a tree of tasks and decisions that forks only at decision incoming arrows in with multiple where a concurrent subtasks. A dotted circle indicates where a signal is generated. Emphasized circles, but also task symbols without incoming arrows, indicate the initial elements, while an unemphasized circle without any incoming arrows marks the beginning of a (sub)programme that will be performed only when some exceptional situation occurs.

Note that when analyzing a task structure, the task diagrams provide some indication of the complexity of the coordination problem. They essentially provide a criterion to decide whether a task should be considered structured ill-structured. In Chapter 2 we stated that a task - being the process of solving a problem - is structured when there exists some programme that describes how to solve that problem. Within the context of task analysis we can be more specific:

Definition 3.10

A task $t=\left(D_{t}, T_{t}\right)$ is structured if and only if the following four conditions are met:

1. if $T_{t} \neq \varnothing$, each subtask $t_{\mathrm{i}} \in T_{t}$ is structured;

2. if $T_{t}=\varnothing$, each decision $t_{\mathrm{i}}$ can be made algorithmically;

3. for each pair $\left\{t_{\mathrm{i}}, t_{\mathrm{j}}\right\} \subseteq T_{t}$ such that $\mathrm{i} \neq \mathrm{j}, t_{\mathrm{i}} \| t_{\mathrm{j}}$ only if $t_{\mathrm{i}}$ and $t_{\mathrm{j}}$ are truly independent, meaning that they require no coordination;

4. task $t$ cannot lead to confusion.

Here ends our formal definition of decision structures and task structures. We must emphasize that the rigor of our definitions is deceptive, because there are no formal means to identify the decisions and tasks of some problem P. For all our definitions, "decision" and "task" have essentially remained the same fuzzy concepts from Section 2.3. But the purpose of these definitions has never been to allow us to analyze the properties of decision structures and task structures the way Petri nets can be analyzed. All that we wanted to achieve is to find some way to represent problem-solving programmes in terms of tasks, decisions, and information in such a way that they can be studied, improved, and where possible supported by means of information technology.

In the remainder of this section we shall describe the process of task analysis. Specific issues we have not dealt with yet are the connection between the decision structure and the task structure of a particular problem, and between task structure and the empirical model of the problem situation.

The fundamental relation between a decision structure $\mathcal{D}_{\mathrm{P}}$ and a task structure $\mathcal{T}_{\mathrm{P}}$ of a problem $\mathrm{P}$ is that the collection of decisions $V$ in $\mathcal{D}_{\mathrm{P}}=(V, E)$ should be a subset of the collection of all decisions that are part of the tasks in $\mathcal{J}_{\mathrm{P}}$. More specifically, $V$ should be identical to this collection minus all coordinating decisions:

\section{Definition 3.11}

A decision structure $\mathcal{D}_{\mathrm{P}}=(V, E)$ and a task structure $\mathcal{T}_{\mathrm{P}}=\left(\mathcal{H}_{\mathrm{P}}, \mathcal{F}_{\mathrm{P}}\right)$ are said to relate to the same problem $\mathbf{P}$ if and only if:

$$
V=\bigcup_{\substack{t \text { in } \mathcal{T}_{\mathrm{P}} \\ T_{t}=\varnothing}} D_{t}
$$

The edges of a decision structure are determined by the influencing and affected part of decisions, which are modeled as information. Each information item 
relates to some variable in the empirical model, or to some expression containing such variables. Demons are in fact logical expressions that indicate which changes in the real system that is modeled by the empirical model require specific decisions to be made. By associating demons with its edges, a decision structure obtains dynamic properties similar to the extended Petri nets described by Kung and S $\phi$ lvberg (1986). From this perspective, decisions are transitions, while information objects constitute the places. For the moment we say without evidence that it is possible to study the dynamics of a decision structure without using the flow relation specified in a particular task structure. By specifying the demon logic, this flow relation (or part of it) is defined implicitly.

By studying the "dynamic behavior" of a decision structure, alternative task structures can be developed. Although these task structures may have the same leaf tasks, their task hierarchy and flow relation may be completely different. The only restriction is that the behavior of a task structure remains consistent with the behavior of the decision structure.

We do not mean to say that, once a decision structure has been determined, it constitutes the reference for task structures, for the only true reference is reality, and the thing closest to it is the empirical model of the problem situation. A decision structure and a task structure are two different representations of a solution to the problem. A decision structure specifies in declarative way which decisions have to be made under given circumstances, which information should be used, and also which support should be provided. A task structure is procedural, rather than declarative.

In total, task analysis can be characterized as an iterative process, a continuous switching between analysis and design of decision structures and task structures.

Recall that our research question focusses on performance improvement of information workers through information technology, and that we interpret this as creating an "environment" for these information workers that provides support for their problem-solving processes. In this chapter we have chosen for a general model of problem-solving processes. We have indicated how an object representation of a problem situation can be of help when conceptualizing and specifying a problem, and we have presented task analysis as a means to support the solution finding activity. In the next chapter, these ideas on how problem solving can be supported will help us to specify what a problem solving support environment should look like.
A PROBLEM SOLVING SUPPORT ENVIRONMENT

As human beings, we shape our environment very effectively because we are able to represent the outer world symbolically, to think conceptually, and to communicate our symbols, concepts and ideas.

\subsection{INTRODUCTION}

In the previous chapter we described how problem solving can be supported through object representation and task analysis. These techniques are feasible only with adequate computer support. If precedence graphs and multi-level flow diagrams were to be constructed and analyzed by hand, using pencil and paper only, task analysis would be ineffective due to time constraints. Similarly, the "translation" of a conceptual model - which itself can very well be developed on paper - into a simulation-type empirical model is impossible without computer support.

Our objective is to design an environment in which decision makers can solve problems faster and more effectively. In this chapter we give the functional specifications of such a problem solving support environment (PSSE). Following Van Schaik and Sol (1986), we use the word "environment" in preference to "system". Firstly because it conveys the image of a decision make surrounded by - and interacting with - people and computers, his phone, the papers on his desk, and so on. Secondly, because the popularity of the labels MIS, DS $S$, ES etcetera makes that "system" is too easily associated with a single box filled with hardware and software.

In determining the functionality of a PSSE we make use of our task analysis technique. We view the process of problem solving as described in Section 3.1 as the task "Solve problem". Analysis of this task provides us with a task structure that gives a more detailed picture of the general process of problem solving. The decision structures of the leaf tasks of "Solve problem" indicate what kind of information a PSSE should provide to a decision maker in the course of his problem-solving process. Thus, task structure and decision structures together form the basis for the functional specifications of a PSSE.

We have restricted ourselves to the case in which there is but a single decision maker. This means that the task structure in this chapter does not contain concurrent tasks, nor is there any need for coordinating mechanisms involving "signals". It also means that we do not investigate in detail how this concurrency will affect the architecture of a PSSE.

The remainder of this chapter is organized as follows: In Section 4.2 we present the result of applying task analysis to the process of problem solving. In Section 4.3 we describe the functionality of a PSSE. In particular, we give the 
specifications of the support component of the leaf decisions that have been identified while performing task analysis. In Section 4.4 we discuss a number of implementation issues. We end this chapter in Section 4.5 with a summary and some concluding remarks.

\subsection{ANALYSIS OF THE PROBLEM SOLVING TASK}

In this section we show how task analysis can be applied to "the problem of solving a problem". In order to minimize the risk of losing one's way among the numerous subtasks and their graphical representations, this section has been organized in accordance with the task hierarchy of "Solve problem" depicted in Figure 4-1. We will traverse this tree in a depth-first fashion, discussing each of its four subtrees in a separate subsection.

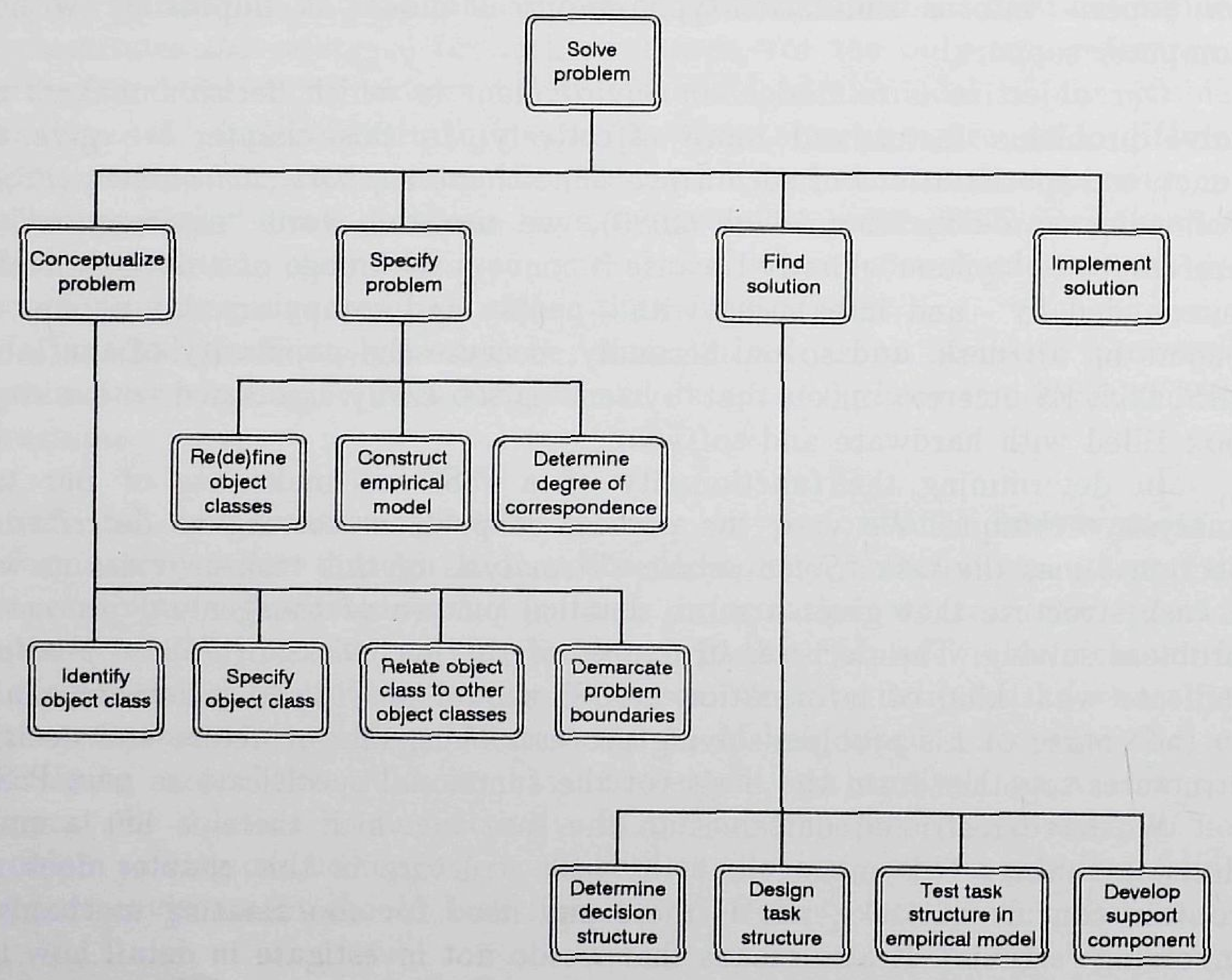

Foure 41. The task hierarchy of the problem solving task
Basing ourselves on Figure 3-1 on page 29, we describe the task "Solve problem" in terms of four subtasks: "Conceptualize problem", "Specify problem", "Find solution", and "Implement solution". The correspondence check and the consistency check in Figure 3-1 are part of the coordination mechanism, since they directly affect the sequence in which the subtasks of "Solve problem" are performed.

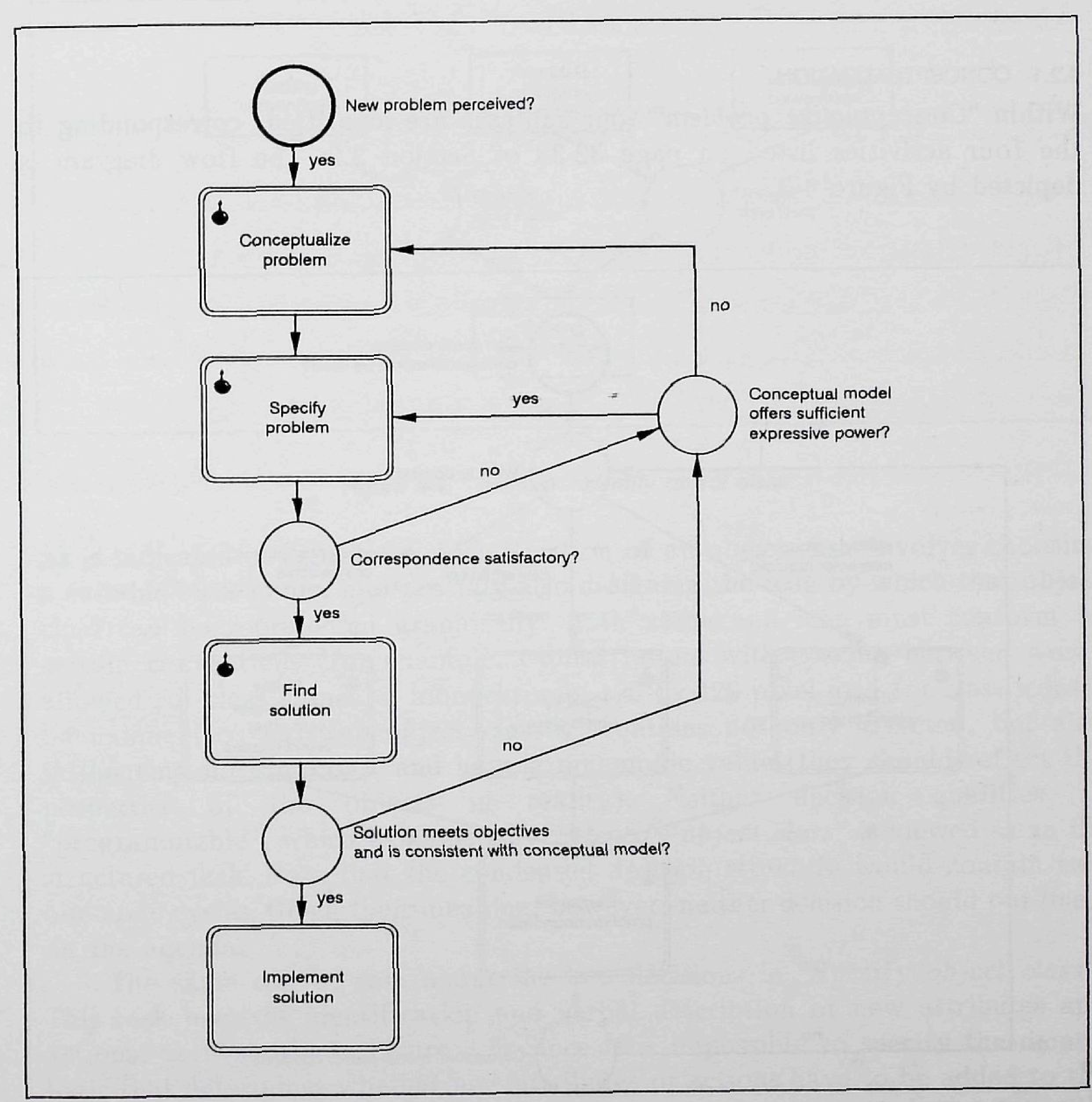

Fgure 42. The task "Solve problem" 
In the task diagram depicted by Figure 4-2 both checks are represented as coordinating decisions. Their outcome determines whether the conceptual or empirical model has to be modified. Notice how the coordinating decision "Conceptual model offers sufficient expressive power?" differentiates between the loop "conceptualization- specification-correspondence check" and the loop "specification-solution finding- consistency check" in Figure 3-1. Each of the four subtasks can in turn be described in terms of decisions and subtasks.

\subsubsection{CONCEPTUALIZATION}

Within "Conceptualize problem" four subtasks are identified, corresponding to the four activities listed on page $32-33$ of Section 3.2. The flow diagram is depicted by Figure $4-3$.

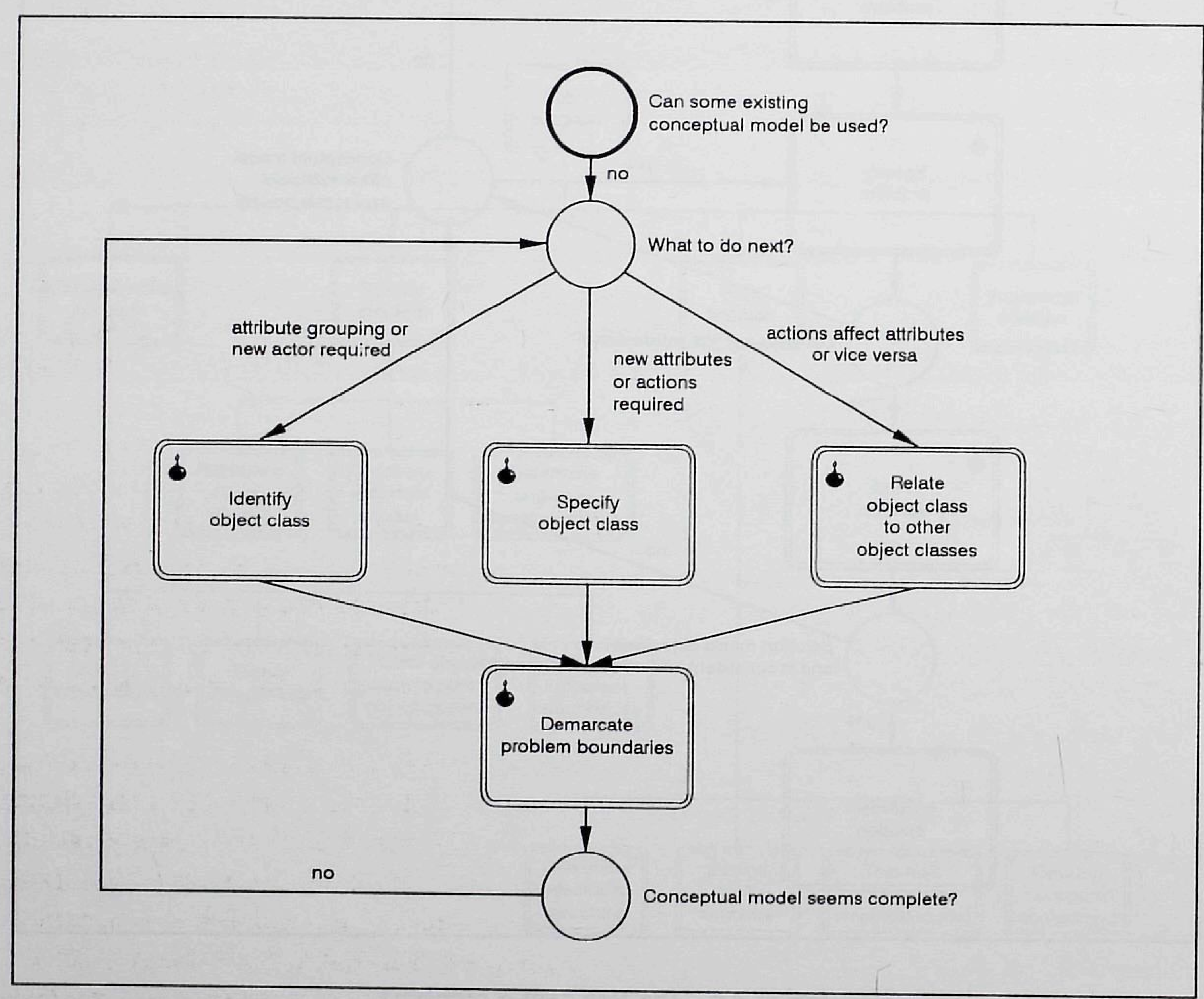

Figure 43. The task "Conceptualize problem"
The fact that there is no specific order to the first three activities is reflected by the way in which coordinating decision "What to do next?" can put any one of the corresponding subtasks on the agenda as long as the conceptual model seems incomplete, a condition that becomes apparent during the problem demarcation activity.

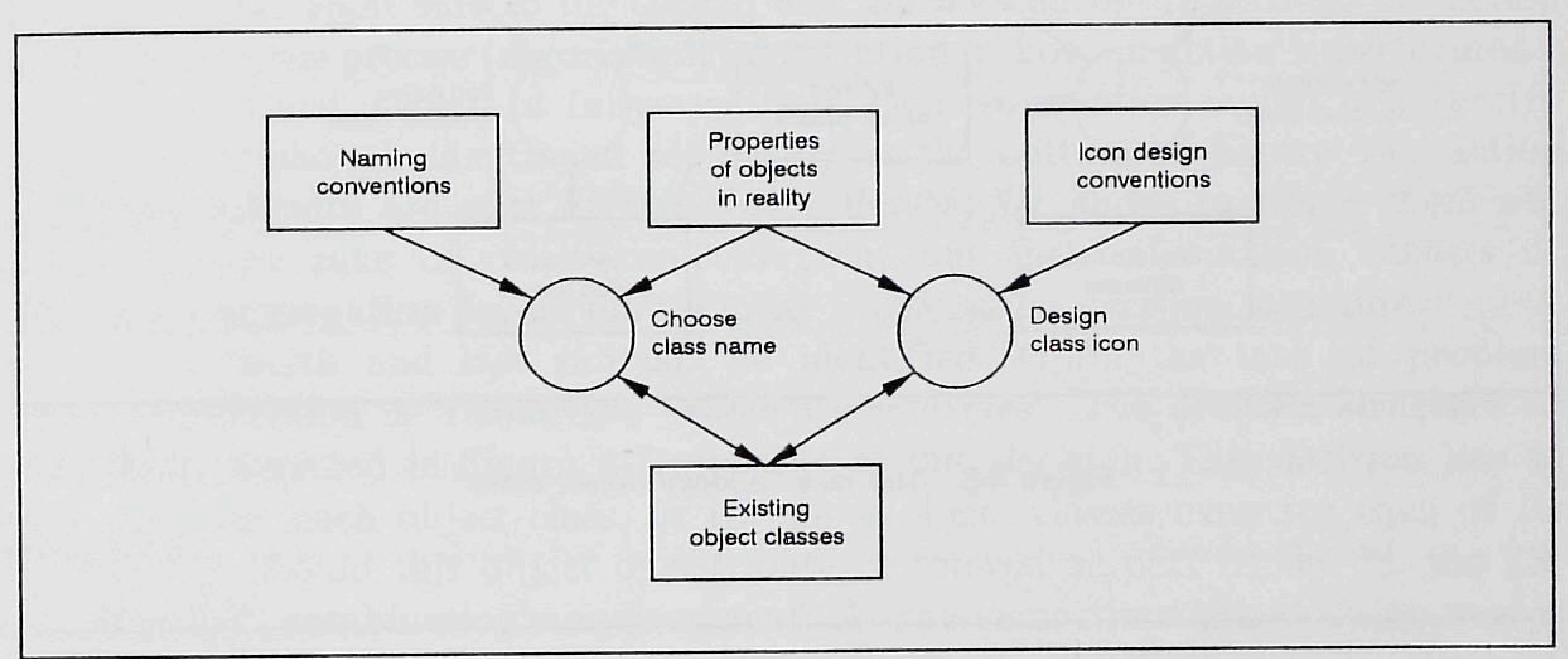

Figure 4-4. The task "Identify object class"

As is indicated in Figure 4-4, identification of an object class involves choosing a suitable class name, and possibly also designing the icon by which that object class can be represented graphically. Both name and icon must conform to certain conventions (for example, capital letters with spacing between words allowed for class names, a monochrome, 120 by 120 pixel grid for class icons), be unique (so "Existing object classes" contains not only affected, but also influencing information), and have a mnemonic value (they should reflect the properties of the objects in reality). Neither decision qualifies as "programmable", which explains why "Identify object class" is viewed as an illstructured task. Note that the condensed decision structure would contain two one-node cycles. Given their meaning, however, neither decision should put itself on the agenda.

The same can be said about the two decisions in "Specify object class". This task involves identification and verbal description of new attributes and actions, as is shown in Figure 4-5. Since it is impossible to specify the demon logic that determines whether new attributes or actions have to be added to the object class, neither decision puts itself on the agenda. Instead, it is left to the decision maker to decide whether some object class needs further specification. This coordinating decision can be found in the task structure in Figure 4-3. 


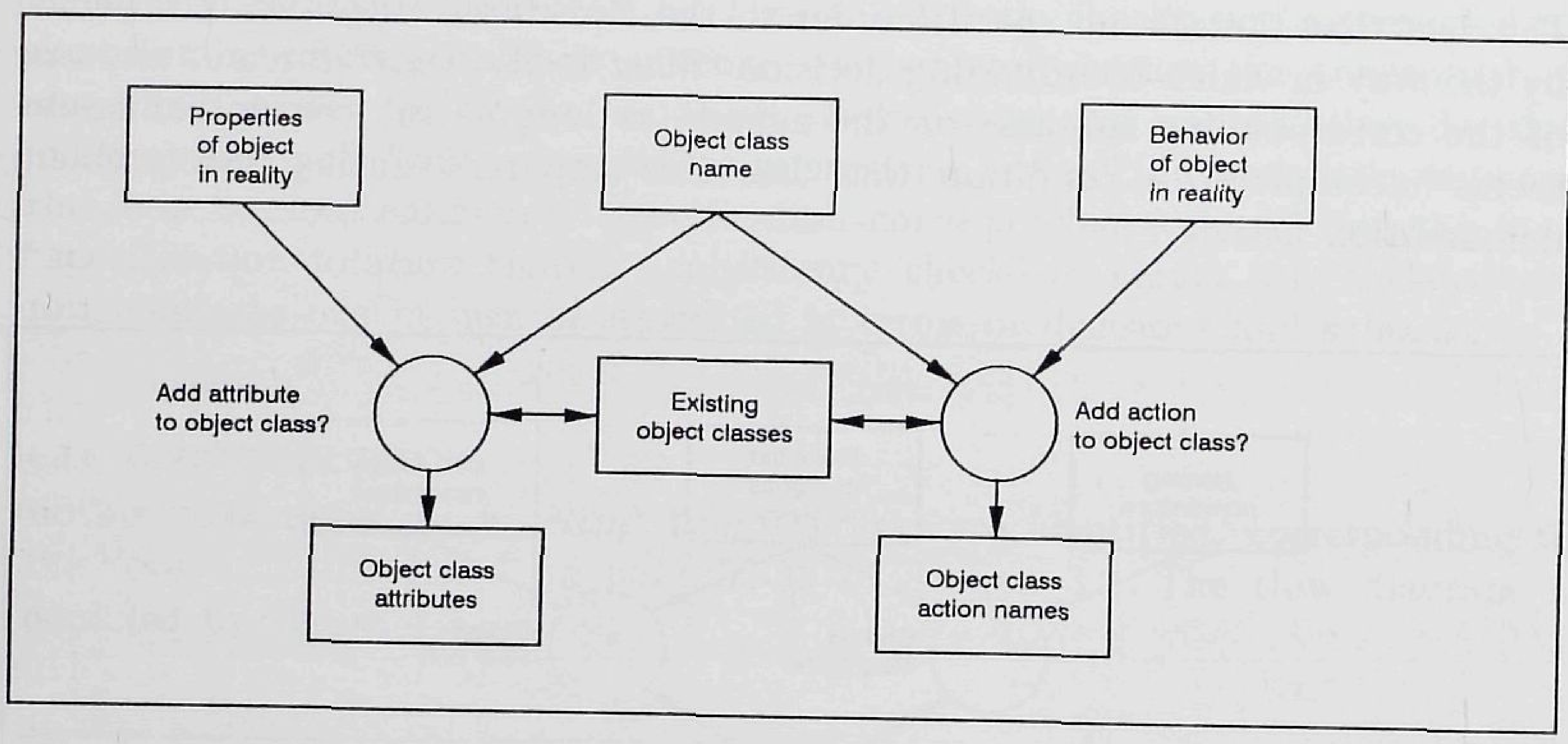

Figure 4-5. The task "Specify object class"

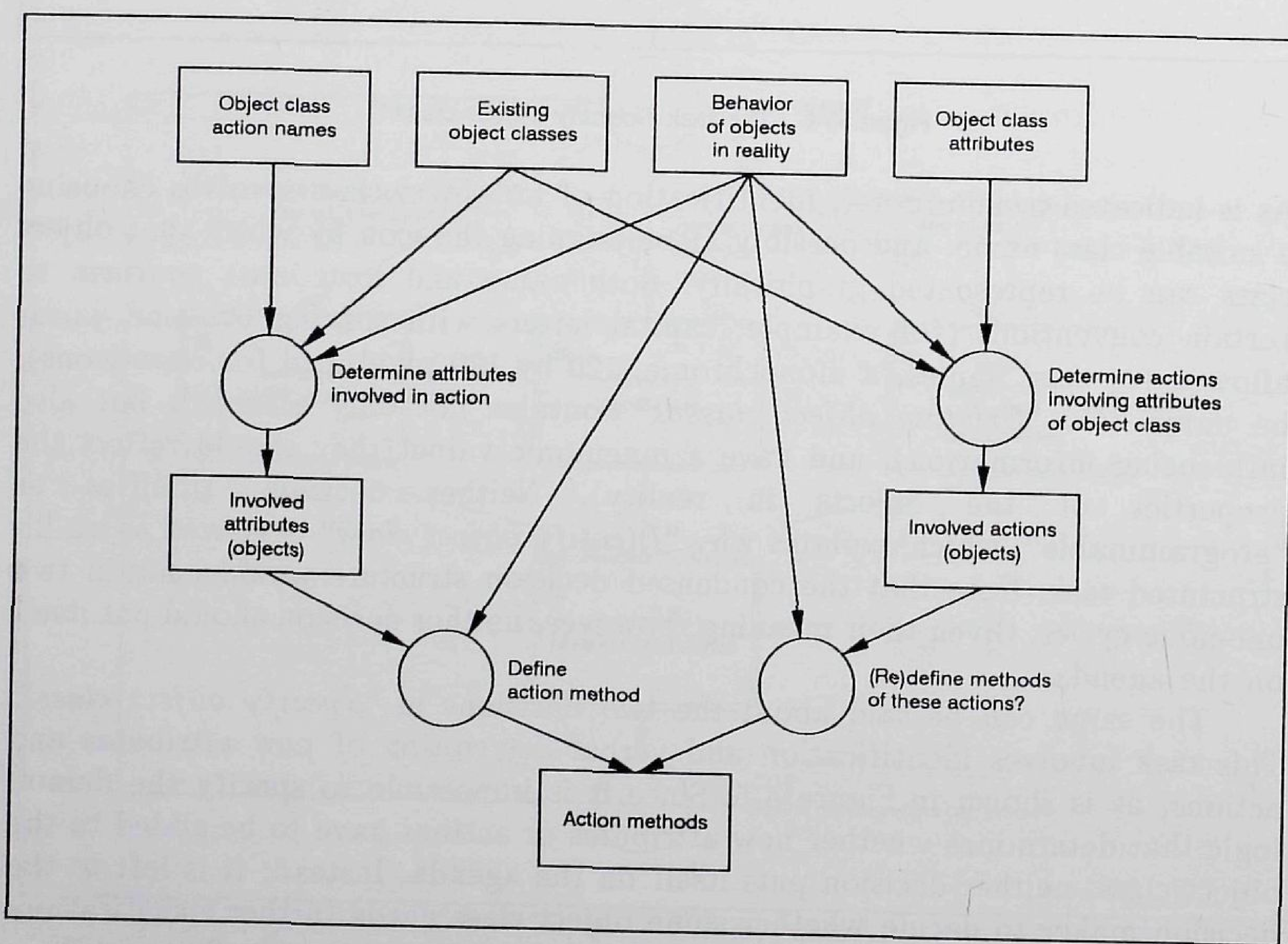

Figuro 46. The task "Relate object class to other object classes"
The decision structure of the task "Relate object class to other object classes" is more complex than the previous two. When inter-relating object classes, one can work in two directions. One can pick an attribute of one object class and subsequently check all object classes for actions that involve this attribute, or one can pick an action and check all object classes for attributes that are involved in this action. The left side of Figure 4-6 corresponds to the first approach, the right side to the second one. During conceptualization, the action "methods" - the precise (algorithmic) description of how an action is performed may be outlined instead of fully specified. Their completion is part of "Specify problem". Although mentioned separately at the bottom of Figure 4-6, action methods actually are part of the object classes. We chose to single them out both for the sake of clarity and to point out that information objects of different aggregation levels may appear in the same decision structure.

The fourth and last subtask we identified within the task of problem conceptualization is "Demarcate problem boundaries". The decision structure of this task, depicted in Figure 4-7, consists of one decision. This decision has to be made for each object class, or for some object classes even for each of its attributes: Should this object or attribute be viewed as part of the IS, the RS, or the IS/RS-combination's environment? At the same time this decision serves as a check whether, on second thought, the identified object class or attribute actually is irrelevant, in which case it should be removed from the conceptual model. Note that we distinguish between "Conceptual model" and "Existing object classes". A PSSE may contain object classes for scores of problem situations, while a conceptual model should only contain those object classes that are relevant to one kind of problem situation.

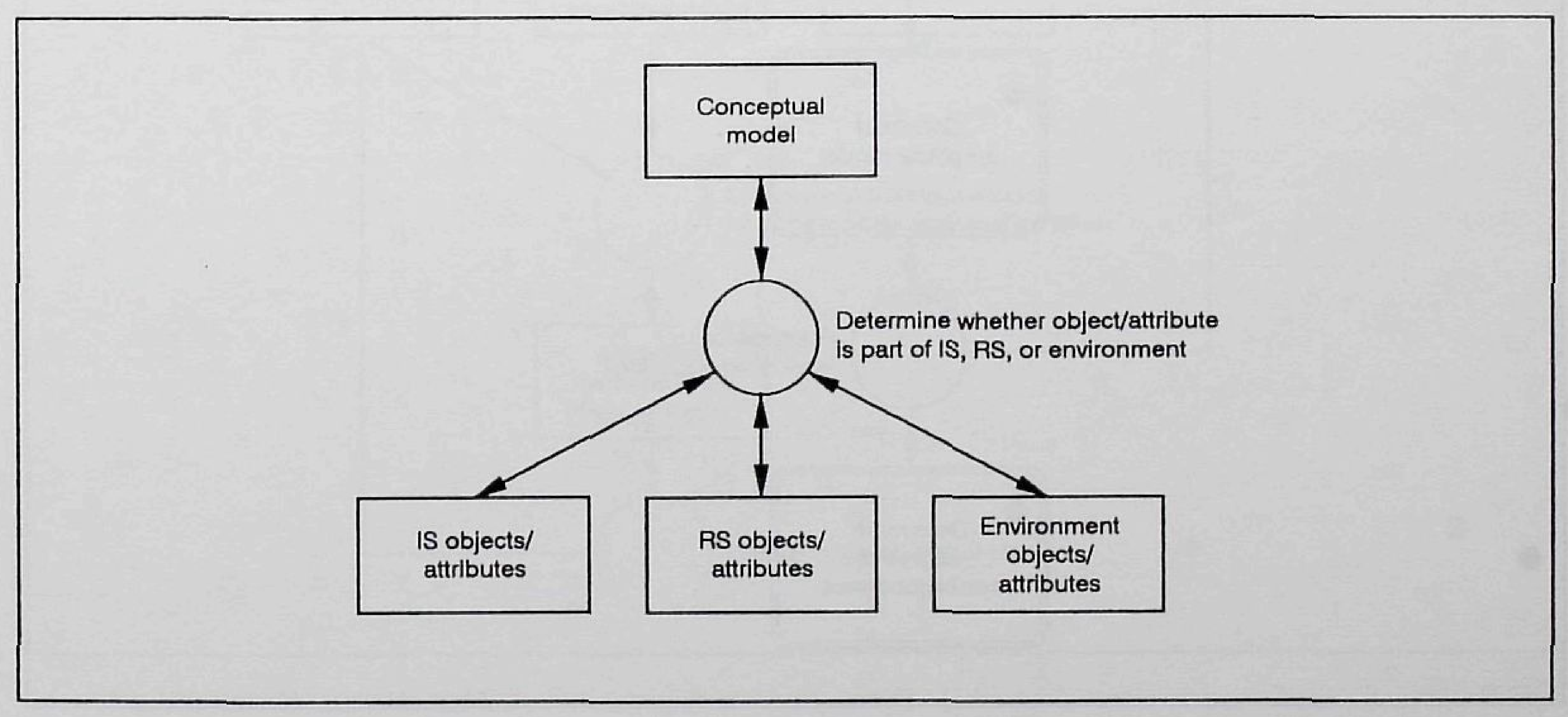

Flgure 47. The task "Demarcate problem boundaries" 
Having determined the decision structure of each of the four subtasks of "Conceptualize problem", we must consider how the decisions we have identified can be supported. It is not surprising that most decisions are influenced by existing object class definitions. Obviously, the decision maker must have easy access to these definitions. When adding a new class, naming conventions should be enforced automatically, and graphical tools should facilitate the creation and modification of icons. The decisions "Determine attributes involved in action of object class" and "Determine actions involving attributes of object class" can be partially automated. Specifying new methods or changing existing ones must be done by the decision maker, but can be facilitated again by providing easy access to class definitions. The decision "Determine whether object/attribute is part of IS, RS, or environment" is difficult to support, but a graphical visualization of the relations between object classes (see for example Figure 5-3 on page 94) often makes it easier to detect actions that "violate" the demarcation rule of the information paradigm. The support component of this decision could therefore consist of a tool that generates such visualizations automatically.

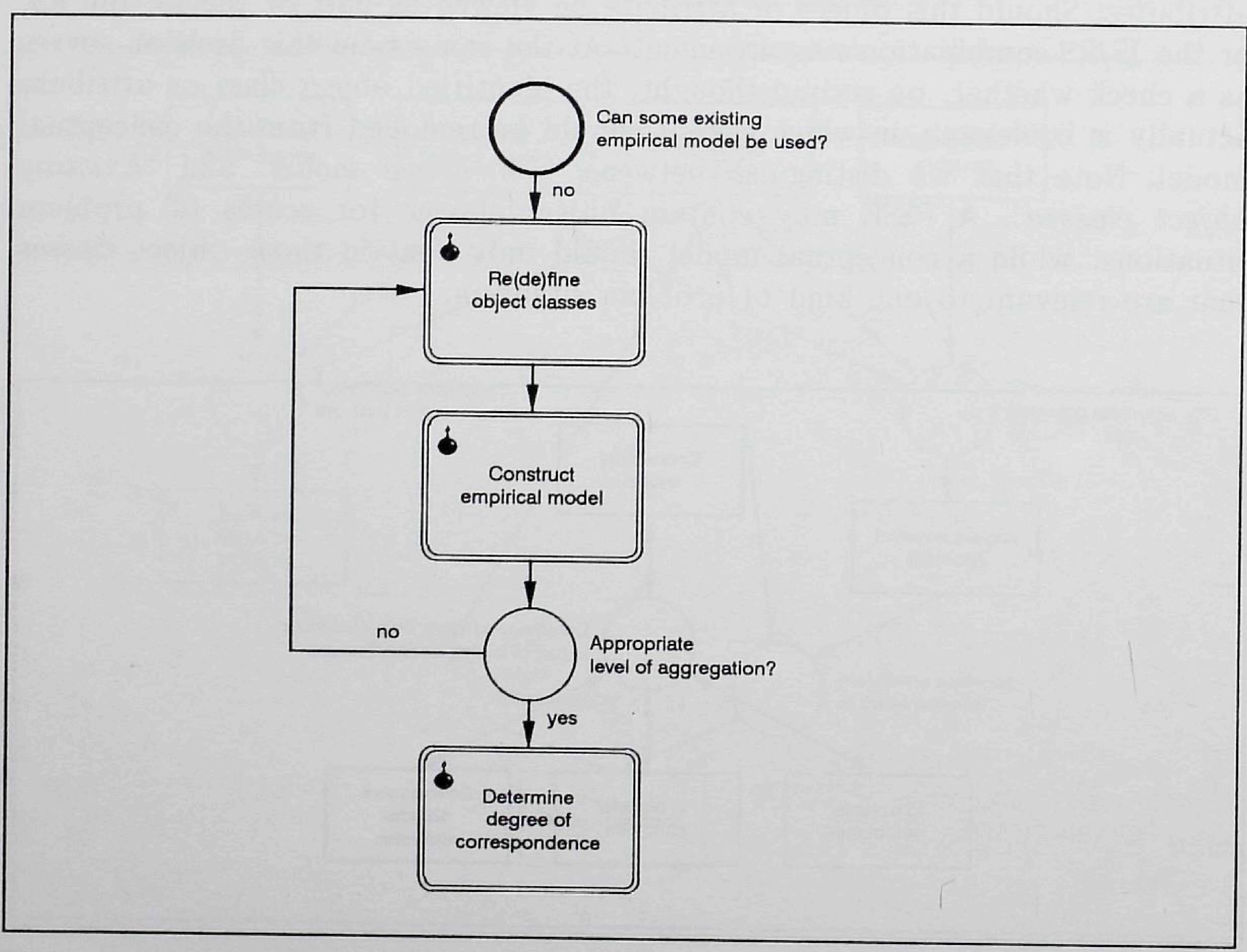

Figure 48. The task "Specify problem
4.2.2 SPECIFICATION

As can be seen in Figure 4-8, the three subtasks we identified within "Specify problem" correspond to the three activities described in Section 3.3. The objective of problem specification is to obtain an empirical model that shows satisfactory correspondence with reality on an appropriate level of aggregation. Although the decision whether the empirical model corresponds closely enough with reality is made on one higher coordination level, the actual task of determining the degree of correspondence is viewed as a part of problem specification. The reason for putting the coordinating decision "Appropriate level of aggregation?" after instead of before the task "Construct empirical model" is that the need for more (dis)aggregation becomes apparent during the actual construction of the model. The decision structures in Figure 4-9 and 4-10 reflect this in the way these tasks are influenced by, but possibly also affect, the aggregation level.

Figure 4-9 shows the decision structure of "Re(de)fine object classes" (see page 35 ). Within this task we identify two decisions. First, existing object classes are scanned for attributes and action methods that, in view of the objectives expressed in the conceptual model, describe part of reality in too much or too little detail. The fact that this decision both affects and is influenced by "Aggregation level" reflects that the aggregation level may change in the course of the problem solving process. The second decision involves changing the object class definitions.

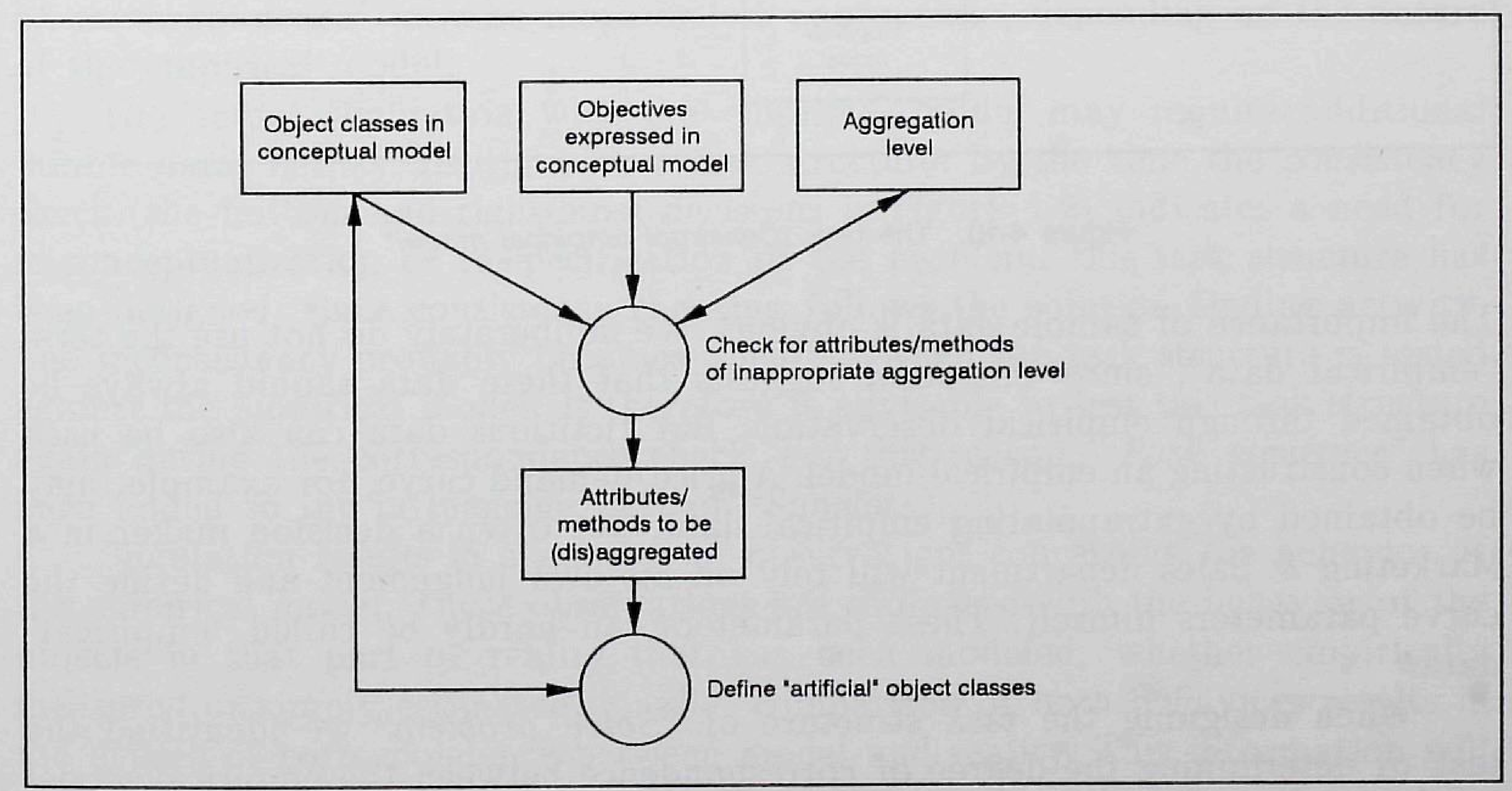

Flgure 49. The task "Re(de)fine object classes" 
The actual construction of an empirical model means creating instances of object classes, which involves determining for each object class the appropriate number of object instances, and for each instance assigning suitable initial values to its attributes. These three activities are represented as decisions in Figure 4-10.

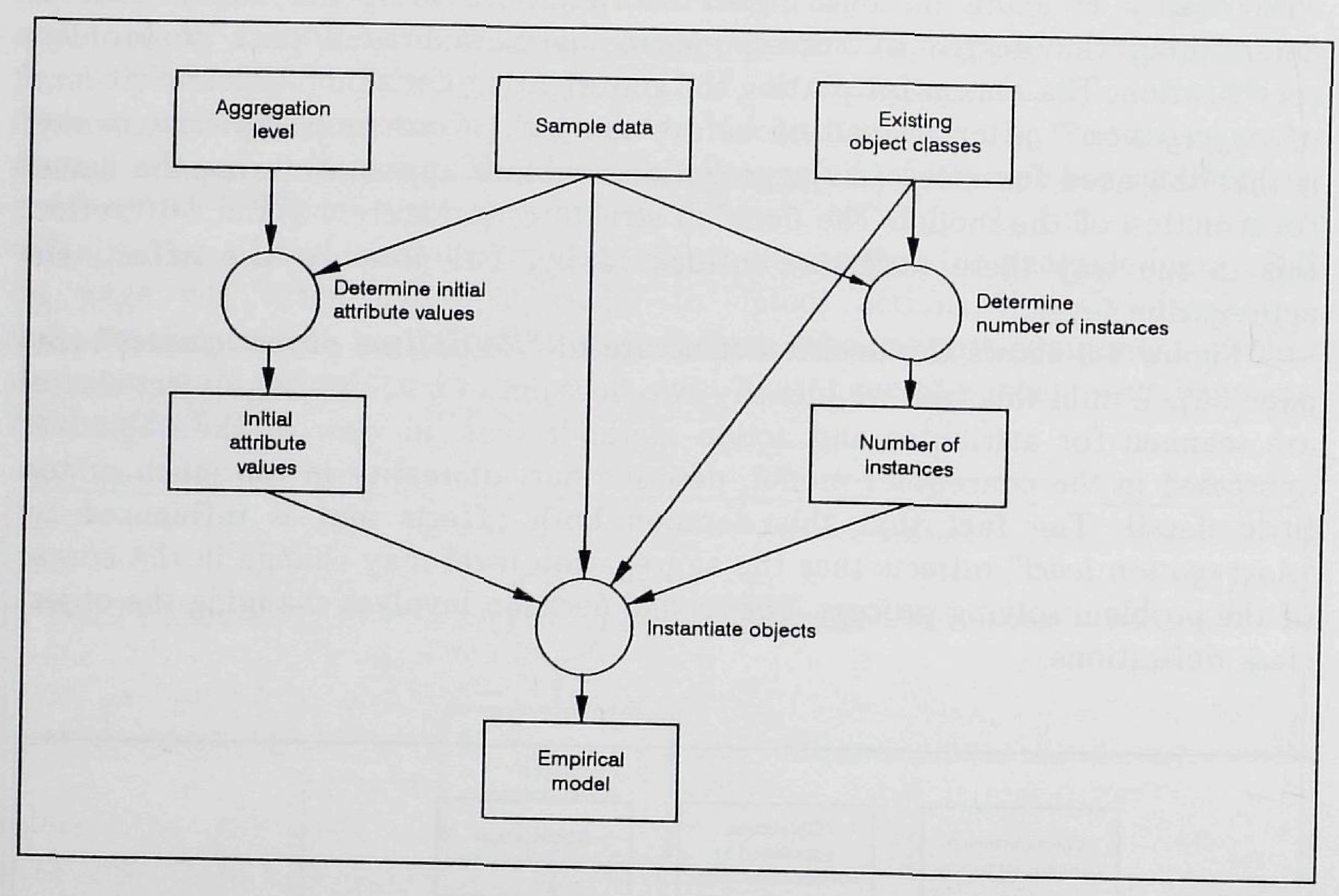

Flgure 4-10. The task "Construct empirical model"

The importance of sample data is obvious. We deliberately do not use the term "empirical data", since this term suggests that these data should always be obtained through empirical observation. But fictitious data can also be used when constructing an empirical model. A price/demand curve, for example, may be obtained by extrapolating empirical data, but often a decision maker in Marketing \& Sales department will rely on his own judgement and define the curve parameters himself. These parameters can hardly be called "empirical" data.

When designing the task structure of "Solve problem" we identified the task of determining the degree of correspondence between the empirical model and reality as a subtask of "Specify problem". Our choice to see it as part of the problem specification activity has been more or less arbitrary. We could very well have put "Determine degree of correspondence" after "Specify problem" in the task diagram of Figure 4-2. However, the simple fact that we represented the consistency check as a coordinating decision only made us decide to put the task of correspondence checking at a lower coordination level.

The decision structure in Figure 4-11 contains four decisions. The choice of confidence level depends on the decision maker's objectives and the nature of the empirical model. For example, if our route planner - who uses a stochastic discrete event-type empirical model of his vans delivering parcels - wishes to compare the anticipated performance of two different route plans, he is likely to set a high confidence level. But if he is interested in the net increase of the firm's total transportation capacity when three new vans are put in operation, a lower confidence level will suffice. The decision maker in the marketing and sales department - who uses a deterministic (spreadsheet-type) forecasting model - will probably have to accept a low confidence level for want of empirical data.

Based on the desired confidence level, the simulation run length (in case of an empirical model with a time dimension) and the number of replications (in case of a stochastic model) must be determined. Note that we use the term "simulation" in a rather broad sense: The first of the two examples in the previous paragraph is a classical example of discrete event simulation, but the second example will more often be classified as a financial modeling problem. In other words, the terminology of the first two decisions in "Determine degree of correspondence" may be more or less appropriate, depending on the nature of the empirical model.

The actual simulation with the empirical model may require additional sample data. It may also involve a task structure: By the time the consistency check (the bottom and right-most decisions in Figure 4-2) indicates a need for re-conceptualization or re-specification of the problem, this task structure has been designed, since consistency checking follows the solution finding activity. The inconsistency probably becomes apparent when the task structure is tested against the empirical model. It therefore is advisable to test this task structure again during the correspondence check. For this reason, "Task structure" has been added to the influencing part of "Simulate".

Simulation results in a number of observations concerning the behavior of the empirical model. These observations are compared with the behavior of the objects in that part of reality that has been modeled, whether empirically measured or simply estimated. Finally, comparison of both behaviors results in the degree of correspondence between model and reality. This information will affect the coordinating decision "Correspondence satisfactory?" in Figure 4-2. 


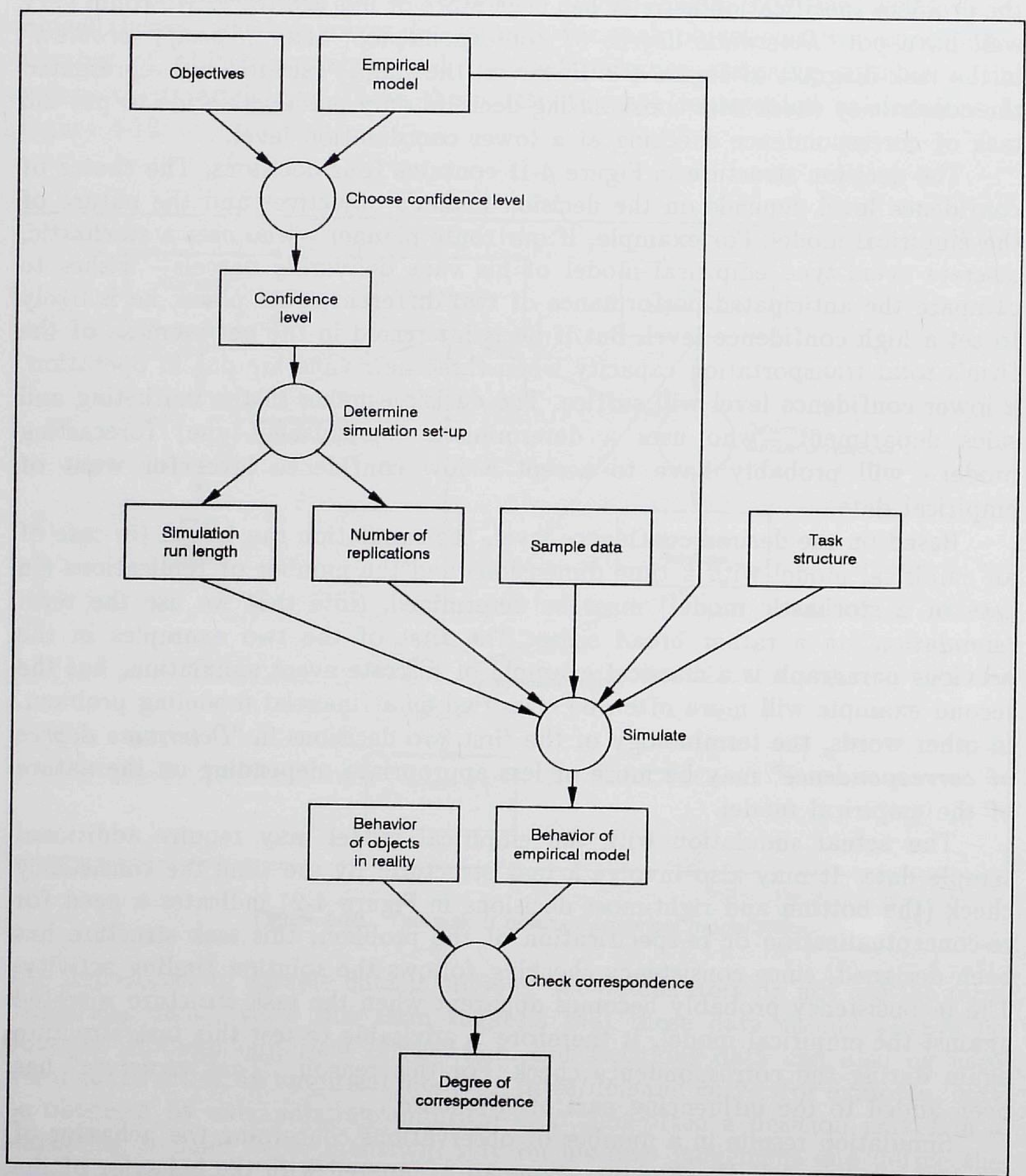

Fgure 411. The task "Determine degree of correspondence"

Again, we can use the decision structure of the three subtasks of "Specify problem" to determine how a decision maker can be supported during the specification activity. The decisions of task "Re(de)fine object classes" mainly require human judgement, and are difficult to support by computer-based means, other than the object class retrieval and editing tools mentioned earlier. When constructing the empirical model, the decisions "Determine initial attribute values" and "Determine number of instances" require tools to analyze sample data, while "Instantiate objects" calls for some means to create and manipulate the collection of object instances that constitutes an empirical model.

Since model construction inevitably means testing and debugging, the decision maker should also be provided with means to trace the model when it is "run". The same tools - both those for (statistical) analysis of sample data and those to trace the behavior of object instances when the empirical mode is tested - can support the task "Determine degree of correspondence".

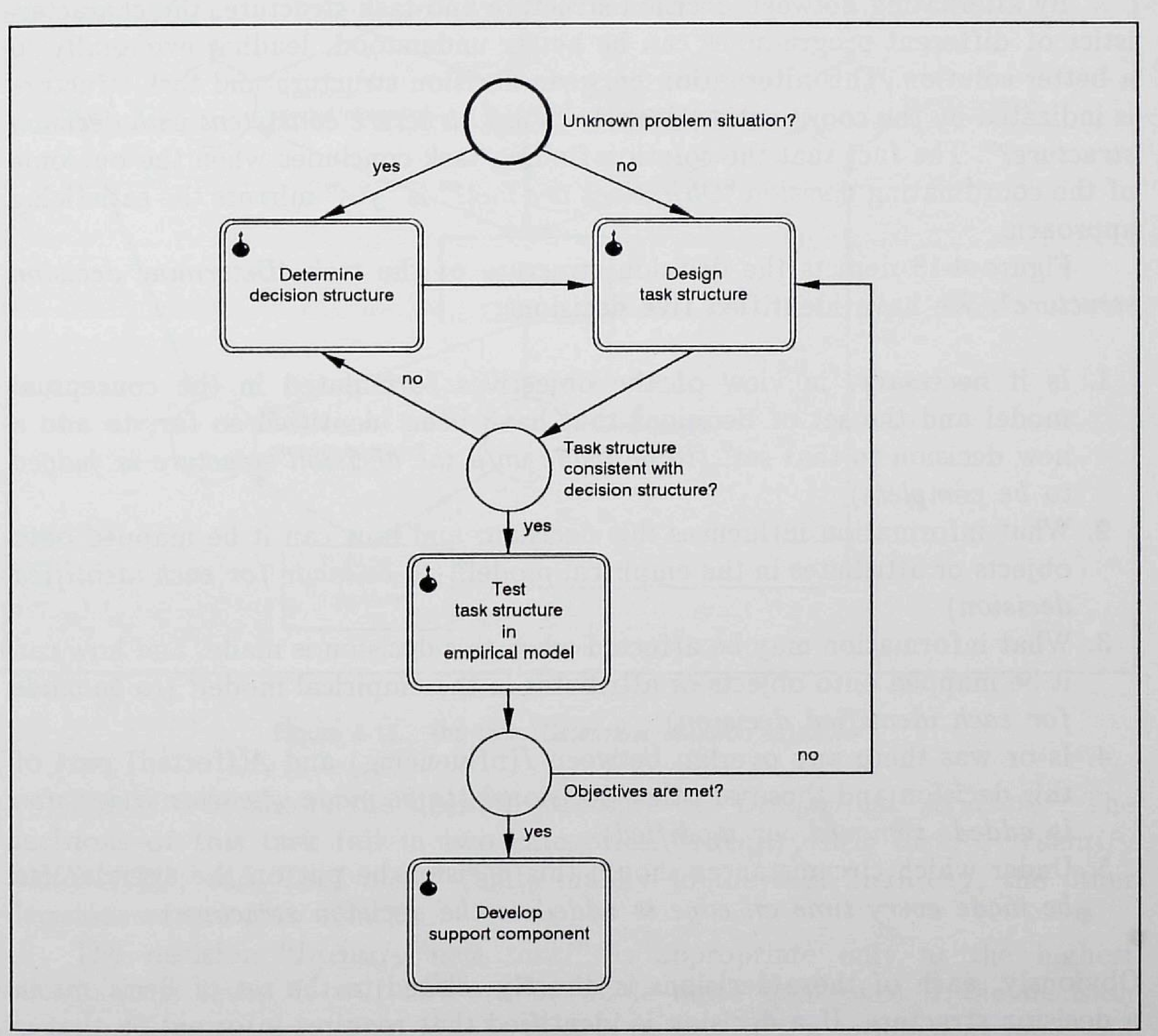

Figure 4-12. The task "Find solution" 
4.2.3 SOLUTION FINDING

As can be seen in Figure 4-12, four subtasks have been identified within the "Find solution" task: "Determine decision structure", "Design task structure", "Test task structure in empirical model", and "Develop support component". Whether one starts by constructing a decision structure or a task structure will depend on the initial "structuredness" of the problem. If similar problem situations have occurred in the past, a first task structure can be specified, based on the way these situations have been dealt with previously. A decision structure is then constructed from the leaf tasks of this task structure. In unfamiliar situations it may be easier to first specify the information that seems to be required in terms of a decision structure, and only then design a task structure.

By alternating between decision structure and task structure, the characteristics of different programmes can be better understood, leading eventually to a better solution. This alternation between decision structure and task structure is indicated by the coordinating decision "Task structure consistent with decision structure?". The fact that the solution finding task concludes when the outcome of the coordinating decision "Objectives are met?" is "yes" mirrors the satisficing approach.

Figure 4-13 depicts the decision structure of the task "Determine decision structure". We have identified five decisions:

1. Is it necessary, in view of the objectives formulated in the conceptual model and the set of decisions that have been identified so far, to add a new decision to that set? (to be made until the decision structure is judged to be complete)

2. What information influences this decision, and how can it be mapped onto objects or attributes in the empirical model? (to be made for each identified decision)

3. What information may be affected when this decision is made, and how can it be mapped onto objects or attributes in the empirical model? (to be made for each identified decision)

4. Is or was there any overlap between $I$ (nfluencing) and $A$ (ffected) part of this decision and those of other decisions? (to be made whenever a decision is added, removed, or modified)

5. Under which circumstances should this decision be put on the agenda? (to be made every time an edge is added to the decision structure)

Obviously, each of these decisions is directly related to the set of decisions in a decision structure. If a decision is identified that requires information that is not represented in the empirical model, this means either that this decision is beyond the scope of the problem under consideration, in which case it should be left out, or that the conceptual model is incomplete. If re-conceptualization is indeed required, this will be detected during the consistency check at a higher coordination level. Whenever an edge is added, one should determine under which circumstances the decision at the end of that edge must be made. If possible, the activating conditions should be added to the decision structure as demons.

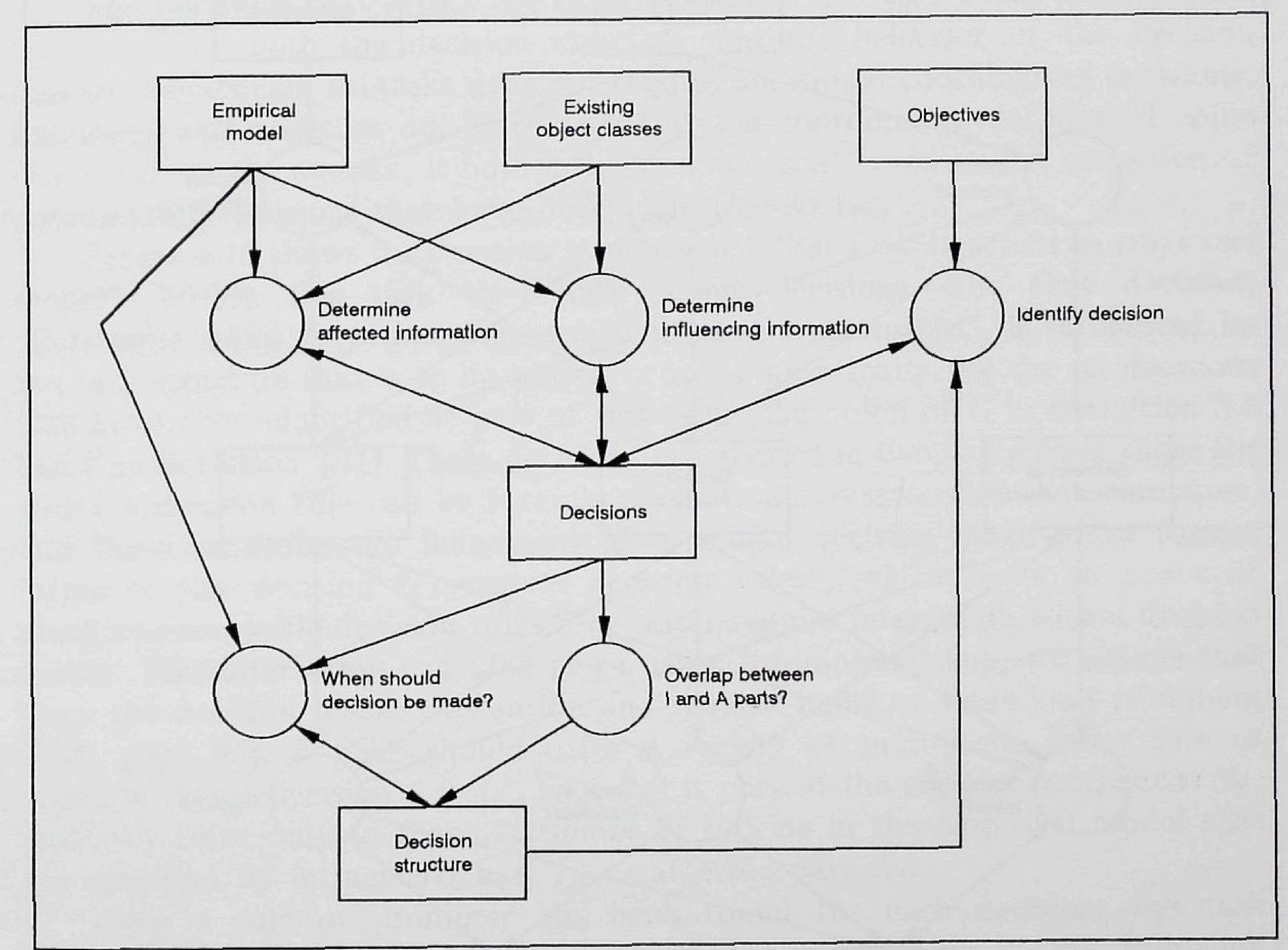

Fgure 413. The task "Determine decision structure"

Figure 4-14 shows the decision structure of "Design task structure". The decisions of this task fall in two categories. "Identify new task?", "Identify subtask(s)?", and "Leaf task?" relate mainly to the task hierarchy, the other decisions to the flow relation.

The decision "Identify new task?" is appropriate only at the highest coordination level. It will have to be made more than once if the decision structure is not a connected graph, that is to say, if the problem at hand can be divided in two or more independent subproblems. 
For each task, the decision "Identify subtask?" will probably be made repeatedly, depending on the properties of the decision structure. It is also influenced by the behavior of the decision makers, since they may make decisions in some particular order that is not revealed by precedence analysis.

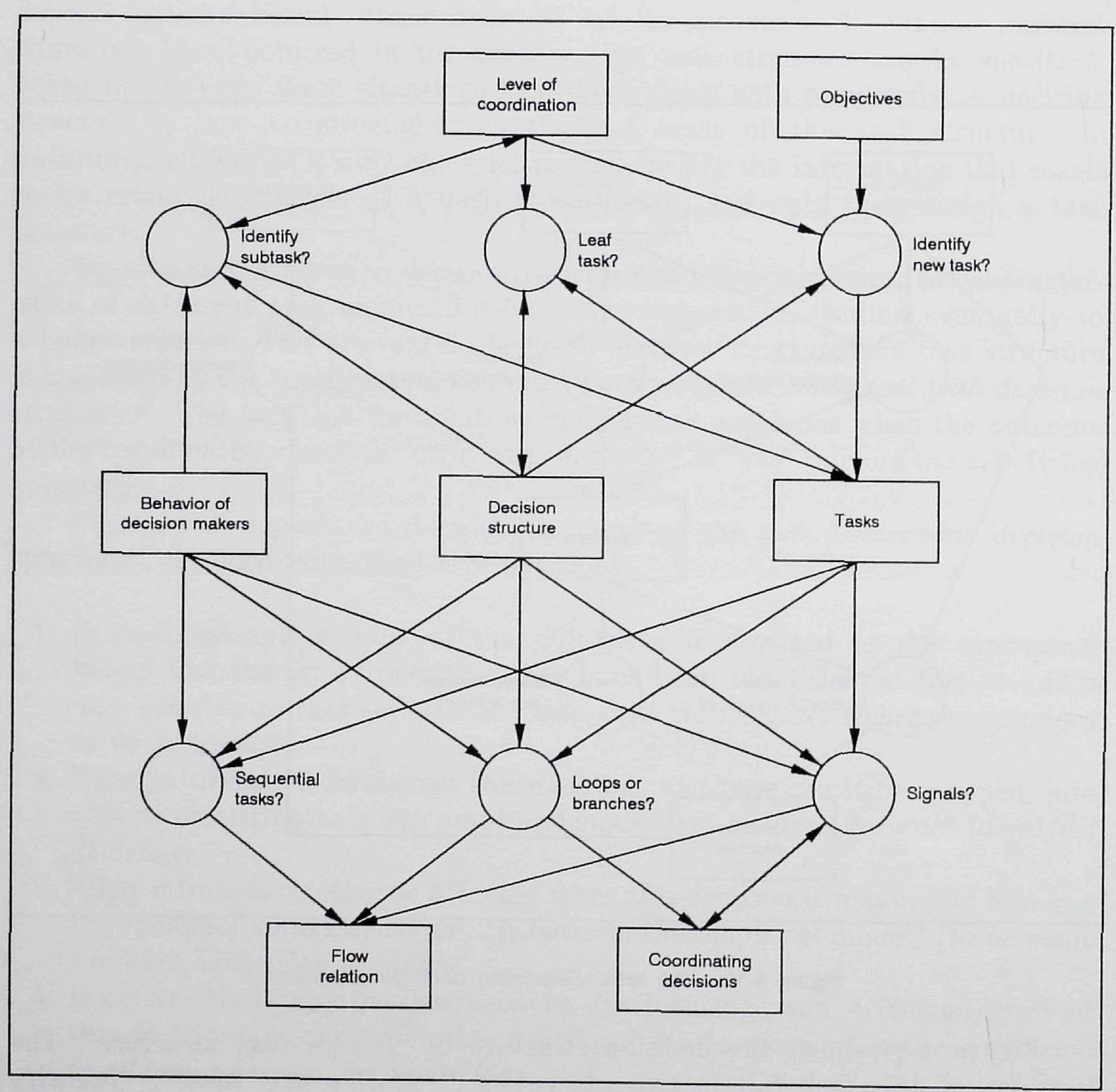

Flgure 414. The task "Design task structure"

The edge to and from "Level of coordination" in the top left corner of Figure 4-13 indicates that by identifying a subtask and subsequently analyzing this task, one reaches a lower coordination level. The outcome of decision "Leaf task?" depends on this coordination level and the complexity of the decision structure of the task being analyzed.

Note that the identification of a subtask may indirectly affect the decision structure if the behavior of the decision makers leads to the identification of a subtask that is relevant to the problem and yet contains none of the decisions in the decision structure. Since a leaf task must contain at least one decision, this decision must be added to the decision structure.

The decisions that affect the flow relation of the task being analyzed are influenced by both the decision structure and the behavior of the decision makers. Sequencing subtasks does not require additional coordinating decisions, but loops and branches do. Since signals put a coordinating decision of some other task on the agenda, it both influences and is affected by the collection of coordinating decisions that have been identified so far.

Figure 4-15 shows the decision structure of "Test task structure in empirical model". Within this task we identify four decisions. The first decision, "Determine which (coordinating) decisions can be automated", is influenced by the task structure that is to be tested, or more specifically, by the all decisions that have been identified as part of that task (the union of $C$ in Definition 3.8 and $V$ in Defintion 3.11). These decisions are divided in two categories: those for which a decision rule can be formulated that can be executed by a computer, and those requiring the judgement of a human decision maker. The former influence the decision "Formulate decision rules", which leads to a set of machine-executable decision rules. The latter require interaction with a decision maker. This interaction can take place using "prompters": support objects that draw the decision maker's attention and request input of some kind (Goldberg 1984, page 41). A PSSE should offer a variety of prompters. Every rule or prompter for a decision $d=\left(I_{d}, A_{d}, S_{d}\right)$ is part of the support component $S_{d}$, and may refer only to those attributes of objects in the empirical model that are specified by influencing part $I_{d}$ or affected part $A_{d}$.

Once a rule or prompter has been found for each decision, the task structure $\mathcal{J}_{\mathrm{P}}$ can be "executed". Task execution proceeds in the following manner: first, the object instances in the empirical model of problem $\mathbf{P}$ are properly initialized, and the agenda mechanism is informed that task $t$ with structure $\mathcal{T}_{\mathrm{p}}$ is about activated. Subsequently, the initial items of $t$ are put on the agenda, and the process described on page 46 commences. Whenever an "automated" decision becomes active, it is made automatically by executing that decision's rule. When other decisions become active, their prompter appears on the screen, asking the decision maker for input. The "execution" halts when task $t$ terminates, that is to say, when the agenda is empty.

The outcome of decision rules and the data entered via prompters change certain attribute values in the empirical model, and thereby affect its behavior. Provided that the empirical model shows good correspondence with reality, this 
behavior will give a fair indication of what would have happened if the decisions had been made in reality. Testing a task structure will doubtlessly require several executions, and the observed behavior of the empirical model may lead to new decision rules. For this reason, "Behavior of empirical model" is affected by "Execute task structure" and influences "Formulate decision rules".

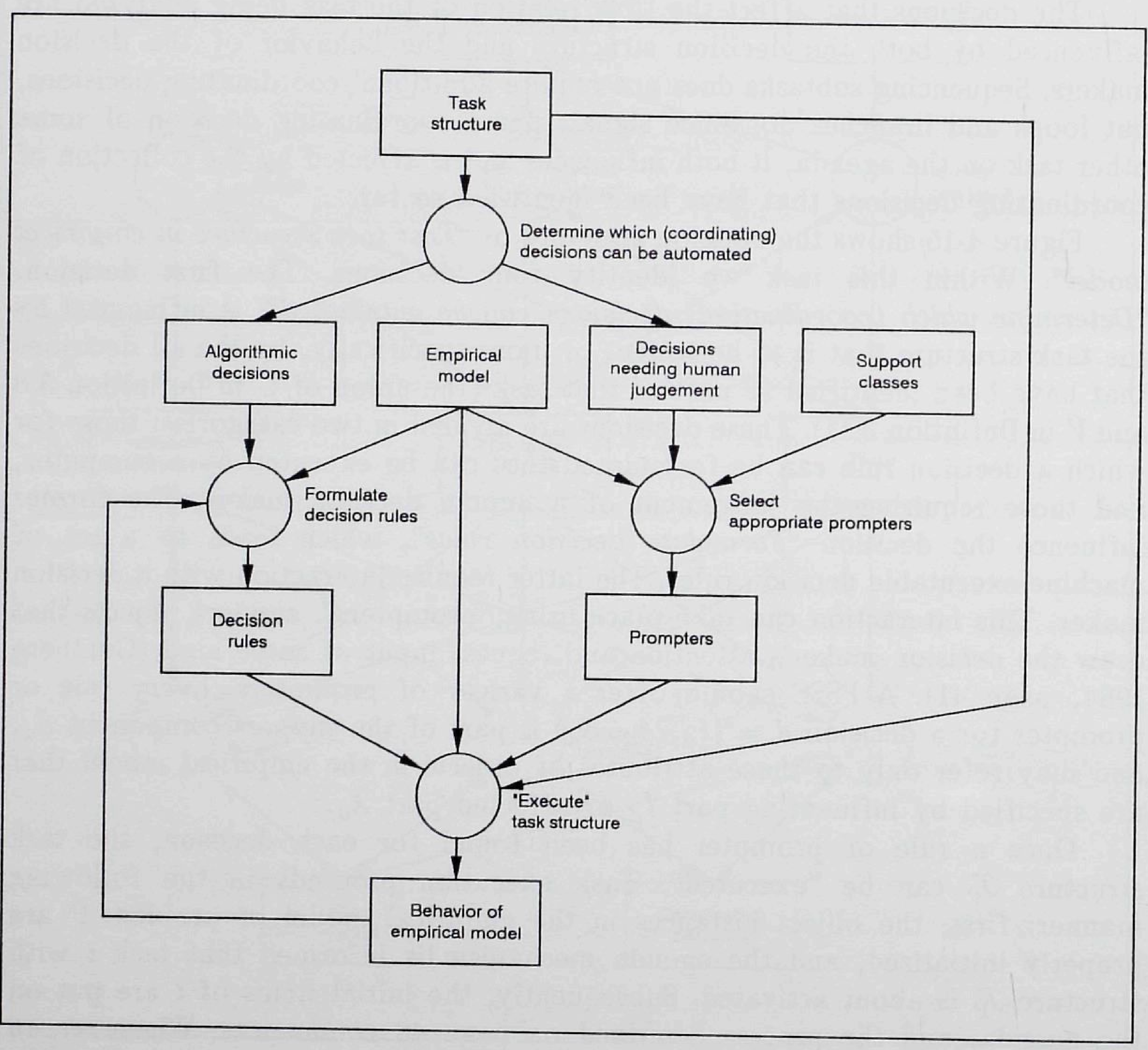

Figuro 4-15. The task "Test task structure in empirical model"

The decision structure in Figure 4-16 outlines the task of developing the support component for a task structure. First, each decision $d$ in the decision structure that could not be automated is considered. The support component $S_{d}$ should present the human decision maker with the information specified by the influencing part $I_{d}$, and it should implement the decision maker's choice by making changes in the affected part $A_{d}$. "Determine required support" results in a set of functional specifications. Based on this set, and using the collection of support classes that have been defined previously within the PSSE, new support objects are developed.

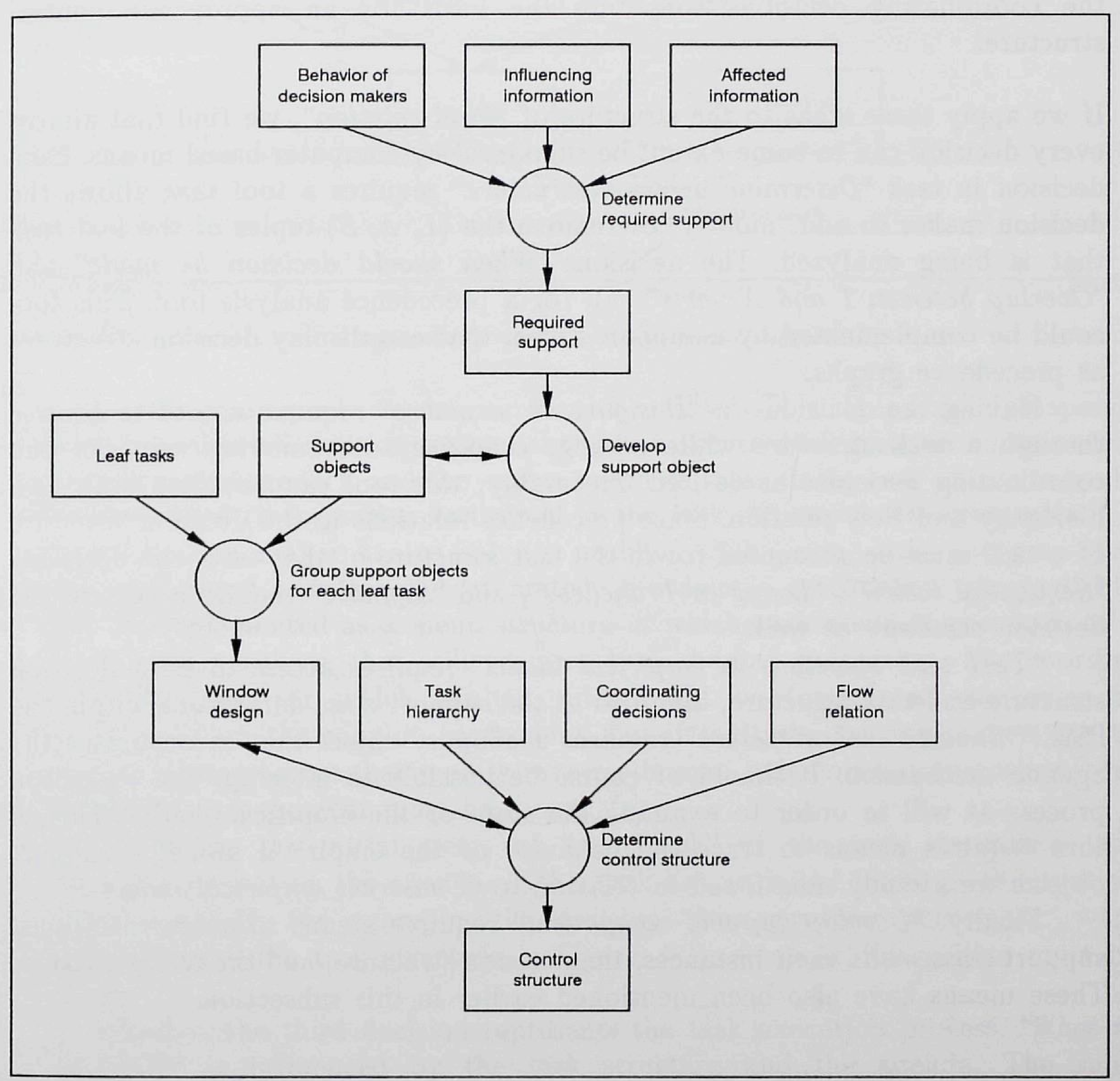

Fgure 416. The task "Develop support component"

Once the support component of each decision has been developed, these components must be integrated. By "integrating" we mean mapping those support objects that should be active simultaneously onto one and the same screen, and 
developing a control structure that allows the decision maker to switch from one screen to another in accordance with the task he has to perform. The third and fourth decision in Figure 4-16 address this issue from the point of view that if a task has been properly analyzed, there will be a one-to-one mapping of its leaf tasks onto screens (support objects of all decisions in a leaf task can be mapped onto the same screen), while the task hierarchy, the flow relation, and the coordinating decisions constitute the basis for an appropriate control structure.

If we apply these ideas to the structure of "Find solution", we find that almost every decision can to some extent be supported by computer-based means. Each decision in task "Determine decision structure" requires a tool that allows the decision maker to add, modify, or remove the $(I, A, S)$-tuples of the leaf task that is being analyzed. The decisions "When should decision be made" and "Overlap between I and A parts" call for a precedence analysis tool. This tool could be complemented by a support object that can display decision structures as precedence graphs.

Making the decisions in "Design task structure" requires a tool to browse through a task structure while adding, changing, or removing subtasks and coordinating decisions as desired. Preferably, this tool can visualize both tas hierarchy and flow relation. Since precedence relations in the decision structure of a task must be accounted for in the task structure of that task, the decisions "Sequential tasks?", "Loops or branches?", and "Signals?" require access to the decision structure as well.

"Test task structure in empirical model" requires access to both decision structure and task structure, and also to the support class definitions within the PSSE. "Execute task structure" requires a support object that incorporates the agenda mechanism. It should of course be possible to interrupt the execution process at will in order to examine the state of the empirical model. This in turn requires means to trace the behavior of the empirical model - support objects we already mentioned in relation to "Construct empirical model".

Finally, "Develop support component" requires means to access decisions, support classes and their instances, the decision structure, and the task structure. These means have also been mentioned earlier in this subsection.

\subsubsection{IMPLEMENTATION}

The task "Implement solution" comes down to executing the problem-solving programme specified by the task structure that has been created during the solution-finding task. Since this is fairly straightforward, we consider the implementation task to be a leaf task within which we identify three decisions. These decisions are depicted in Figure 4-17.

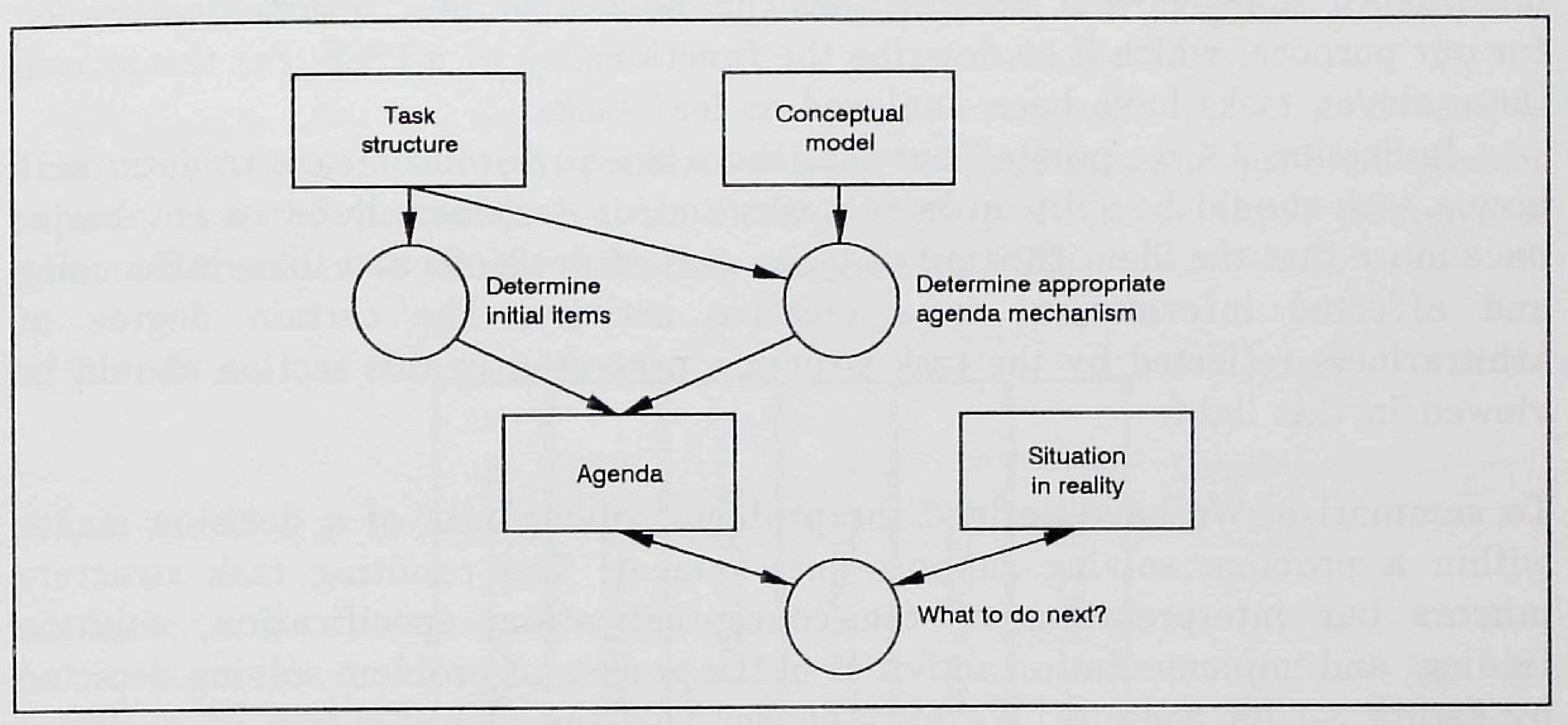

Fgure 417. The task "Implement solution"

First, a suitable agenda mechanism must be chosen. This choice mainly depends on the number of decision makers that are involved (information contained in the conceptual model), and the extent to which tasks in the task structure could be automated (information contained in the task structure). For example, if a single decision maker has to solve a problem that - using task analysis - has been structured and largely automated, the agenda mechanism can probably best be implemented as a menu structure in which each menu corresponds to a coordinating decision that still requires some human judgement. The coordinating decisions for which explicit rules could be formulated can even be "hard-wired" in the support component. But if multiple decision makers are to execute a programme that mainly requires human judgement, some other form of agenda management must be looked for.

Secondly, the initial items of "root task" of the task structure's task hierarchy are put on the agenda. If this task has no initial items (such situation would occur if, for example, the decision "New problem perceived?" were removed from the task structure in Figure 4-2), some arbitrary item must be chosen.

Finally, the third decision represents the task execution process. "What to do next?" is influenced by the task structure and the agenda. The rules formulated in Definition 3.9 on page 46 determine how "What to do next?" affects the agenda.

In all, our task structure of "Solve problem" comprises fifteen tasks: four on the highest coordination level, and eleven at one lower level of coordination. We could have extended our task structure by describing each of these eleven tasks 
in terms of subtasks, but we find that this makes the description too detailed for our purpose, which is to describe the functionality of a PSSE. For this reason these eleven tasks have been analyzed as leaf tasks.

In Section 3.4 we pointed out that there are no formal means to determine how a task should be split up in subtasks and/or decisions. Here we emphasize once more that the identification of tasks, and of decisions and their influencing and affected information, is a creative activity. The certain degree of arbitrariness reflected by the task structure presented in this section should be viewed in this light.

To summarize: We have defined the problem solving task of a decision maker within a problem solving support environment. The resulting task structure mirrors our interpretation of the conceptualization, specification, solution finding, and implementation activities in the process of problem solving depicted by Figure 3-1 on page 29. We have shown how the identification of decisions and their influencing and affected information helps in finding the appropriate support component for the task "Solve problem". In the next section we will look at this support component in more detail.

\subsection{FUNCTIONAL REQUIREMENTS}

A full-fledged PSSE should be capable of concurrently supporting numerous information workers. However, as we remarked earlier, our study of the functionality that a PSSE should offer has been limited to a single-person environment.

Object-oriented modeling takes a central position in our approach to problem solving: We interpret problem conceptualization mainly in terms of identifying and specifying object classes, while object instantiation and initialization are essential to the construction of an empirical model. Moreover, though the definitions in Section 3.4 do not explicitly call for an object-oriented representation of decision structures and task structures, the dynamic behavior of these structures can be studied best when the effects of decision making become visible as changes in the empirical model. This requires a direct link between the Influencing and Affected information of decisions and the objects in the empirical model (see also page 40 ). Such direct linking can be realized quite naturally when decisions and tasks themselves are represented as objects in the same way the objects in the empirical model are represented. The $I$ and $A$ parts, for example, become attributes of object class DECISION that can refer to instances of any other class. Finally, the support component $S$ can also be modeled in terms of attributes and actions, which makes a complete integration of conceptual and empirical model, task structure, and support component possible.

This integration of problem, problem-solving programme, and problem-specific support that yet preserves a clear distinction between these three elements is what we consider to be the main advantage of a PSSE.

\begin{tabular}{|c|c|c|c|c|c|}
\hline 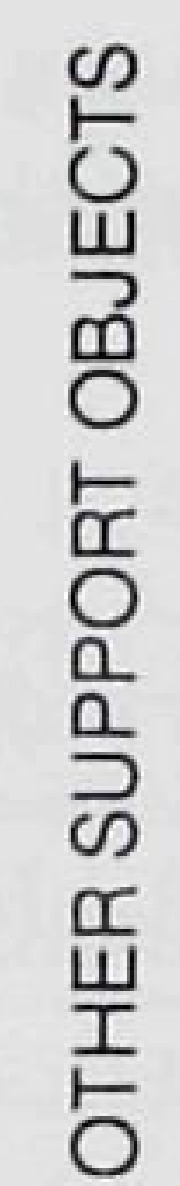 & 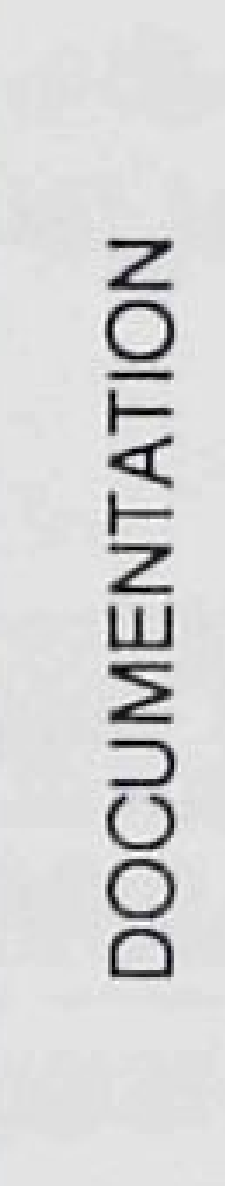 & 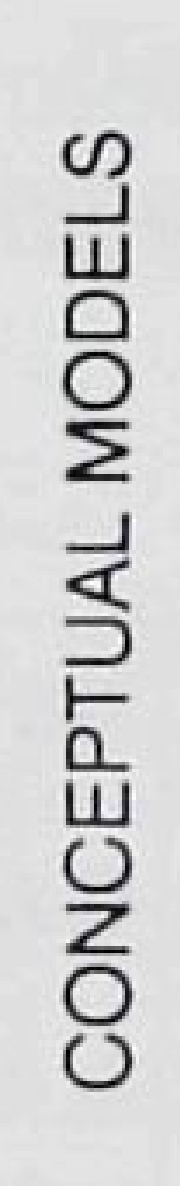 & 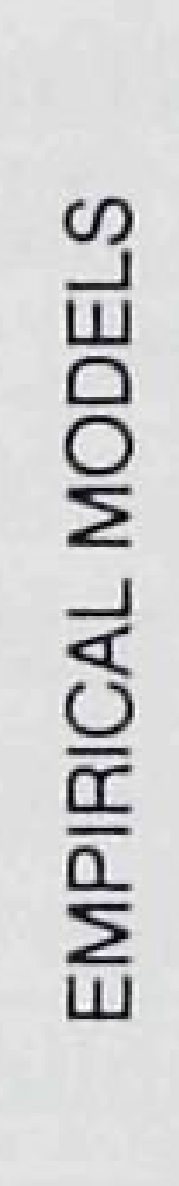 & 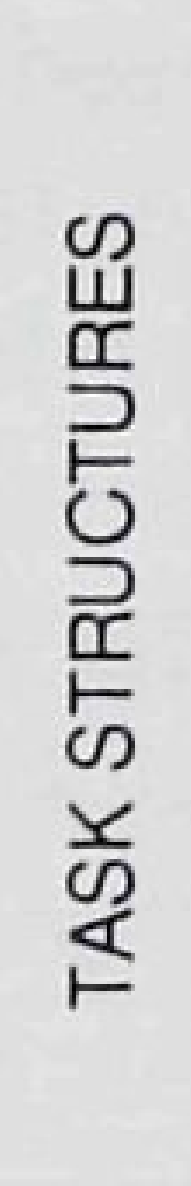 & 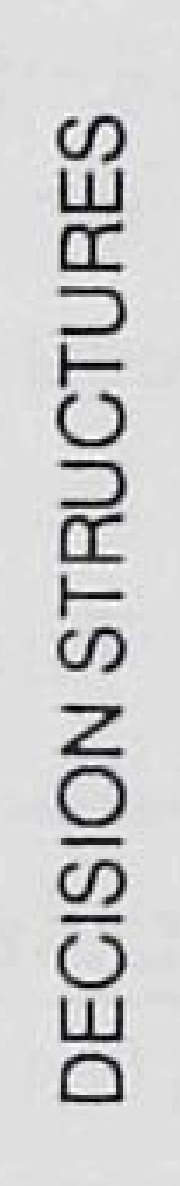 \\
\hline \multicolumn{6}{|c|}{ KERNEL SUPPORT OBJECTS } \\
\hline
\end{tabular}

Flgure 4-18. The global architecture of a problem solving support environment

In view of the vital role of object-oriented modeling in our approach to problem solving, it is difficult to envision an environment that does not have an objectoriented architecture. The fundamental part of such an architecture is what could be called an "infrastructure" of both hardware and software that fully supports object class definition, inheritance of properties, instantiation, communication, etcetera - in brief: object management. Figure 4-18 shows a three-layer PSSE architecture. At the basis lies the object manager that takes care of memory allocation, compilation of object class definitions, instantiation of objects, and input/output. It provides roughly the same functions as an operating system. On top of this object manager the so-called "kernel support objects" are built. These objects constitute the support component of the various subtasks identified in the previous section. They provide a wide range of functions to support problem conceptualization and specification, task analysis, and the implementation of other support objects. The third layer contains the things that can be manipulated by means of the kernel support objects: 
conceptual and empirical models, decision structures, task structures and their support components, and the documentation that should be kept for all objects in the PSSE.

While commenting on the decision structures of the leaf tasks of "Solve problem" we briefly outlined what kind of support can be offered. In this section we shall become more specific. Figure 4-19 indicates which kernel support objects are intended to be used during which leaf task of "Solve problem" (see Figure 4-24 at the end of this section for a cross-reference). Since the support class DOCUMENTATION BROWSER is used during all of the tasks listed below, it is not mentioned explicitly. For the task "Implement solution" no general (kernel) support objects have been developed.

\begin{tabular}{|c|c|}
\hline Leaf task & Kernel support object(s) \\
\hline Identify object class & CLASS BROWSER, ICON EDITOR \\
\hline Specify object class & CLASS BROWSER \\
\hline Relate object class to other object classes & $\begin{array}{l}\text { CLASS BROWSER, MODEL BROWSER, } \\
\text { GRAPH EDITOR }\end{array}$ \\
\hline Demarcate problem boundaries & MODEL BROWSER， GRAPH EDITOR \\
\hline $\operatorname{Re}(\mathrm{de}) \mathrm{fine}$ object classes & CLASS BROWSER \\
\hline Construct empirical model & $\begin{array}{l}\text { MODEL BROWSER, ATTRIBUTE MONITOR, } \\
\text { ACTION LOGGER, STATISTICAL ANALYZZER }\end{array}$ \\
\hline Determine degree of correspondence & ATTRIBUTE MONITOR, STATISTICAL ANALYZER \\
\hline Determine decision structure & $\begin{array}{l}\text { MODEL BROWSER, DECISION BROWSER, } \\
\text { INFORMATION BROWSER, GRAPH EDITOR }\end{array}$ \\
\hline Design task structure & $\begin{array}{l}\text { TASK BROWSER, DECISION BROWSER, } \\
\text { GRAPH EDITOR }\end{array}$ \\
\hline Test task structure in empirical model & $\begin{array}{l}\text { MODEL BROWSER, TASK BROWSER, } \\
\text { ACTION LOGGER, STATISTICAL ANALYZER }\end{array}$ \\
\hline Develop support component & SUPPORT BROWSER, CLASS BROWSER \\
\hline Implement solution & MODEL BROWSER, TASK BROWSER \\
\hline
\end{tabular}

Figure 4-19. The relation between problem solving subtasks and kernel support objects

\section{Documentation browser}

We consider this particular support class first because it is least task-specific. A documentation browser provides fast access to any piece of documentation within the PSSE. In addition to a variety of word processing functions, it should with facilities for document retrieval on keyword, as well as full text search. offer facilities for document retrion maker will use the documentation browser whenever he is looking for information on class definitions, models, task structures, decision structures any object, actually. In the course of problem conceptualization, for example, he will want to know whether conceptual models of (parts of) the specific problem situation he perceives have been made previously. Since these models - or their object classes - may be stored under other names than the decision maker would have chosen himself, a search for specific keywords in the documentation is likely to be more effective than browsing through the (extensive) list of existing object classes.

\section{Class browser}

A class browser provides access to all class definitions in the PSSE. Basically, it constitutes the most direct interface between the decision maker and the object manager. Using the class browser, the decision maker can define new object classes, and modify existing ones by adding new attributes and re(de)fining actions. An important feature of the class browser is that it allows the decision maker to maintain various definitions of the same object class. In the Section 3.1 we mentioned that the same object can be represented on various levels of detail, depending on the problem at hand. As an example we gave the object class VAN. Its attributes and actions related to break-downs in the context of the routing problem are quite different from those in the context of the van replacement problem, but in both cases the same object in reality is concerned. Another important feature is "version management". Whenever an object class is modified, this should not affect any object instance within the environment unless this instance is explicitly "re-classified". As long as there is still some reference made to a specific version of a class definition, it cannot be discarded.

\section{Icon editor}

Since each object class has an icon associated with it, there is a need for a supporting tool with which icons can be created and modified. This icon editor provides functions similar to the Smalltalk-80 FORM EDITOR class (Goldberg 1984, page 120-132). The icon editor in Figure 4-20 is a four-pane window application. The larger of the two graph panes serves a "drawing sheet" in which each icon pixel is magnified 16 times to facilitate editing using the mouse cursor. The smaller one displays the icon full size. Special functions can be activated using the list pane, while the text pane at the bottom displays information concerning the next operation that is expected from the decision maker. 


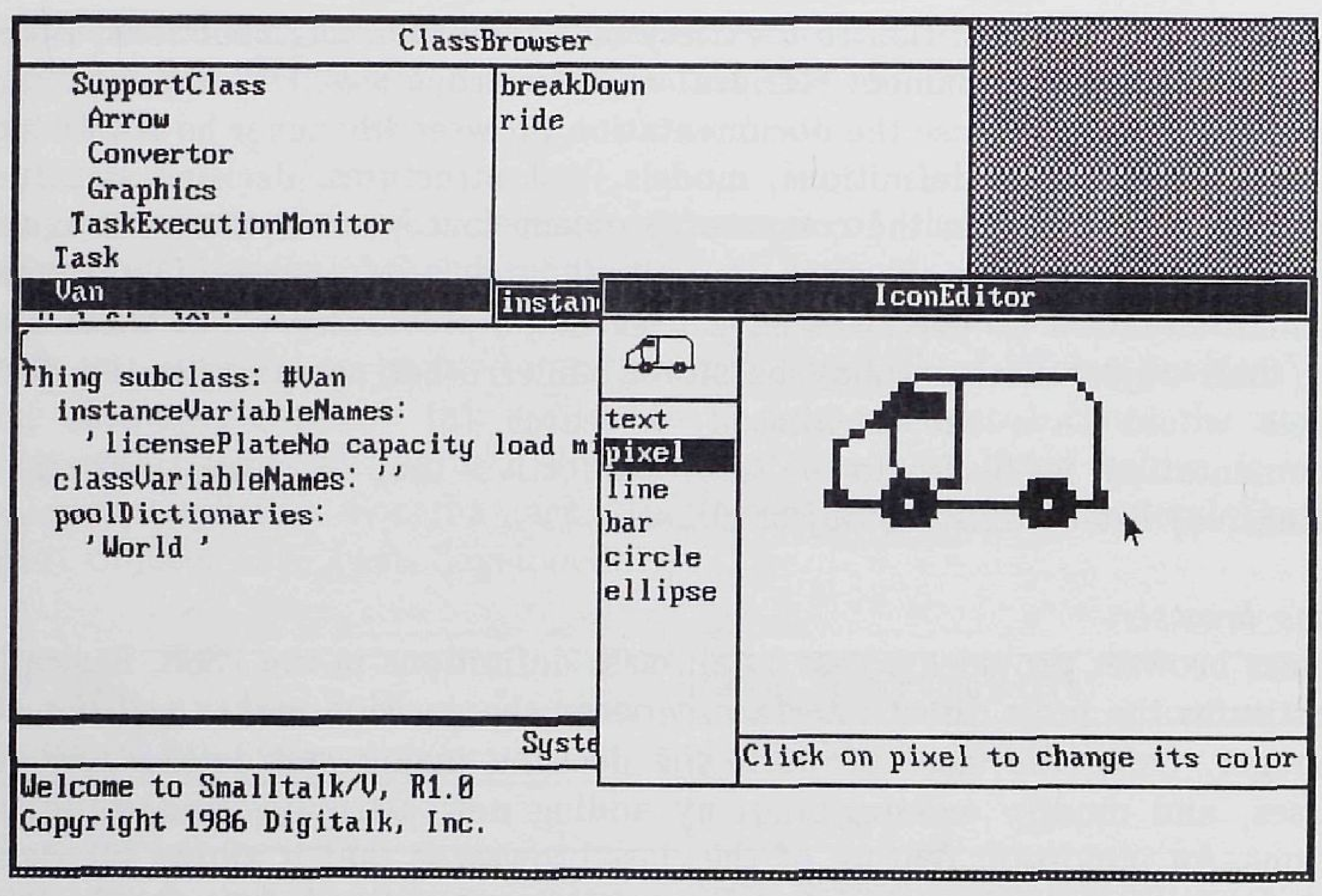

Flgure 4-20. A class browser and an icon editor

Model browser

Instances of this support class allow easy manipulation of both conceptual and empirical models. Although a class browser essentially provides all functions required for the creation and maintenance of object classes - even with multiple definitions of the same class - it is not model-oriented. There still is a need for access to conceptual models as a whole, instead of individual classes. This is the primary function of a model browser as far as conceptual models are concerned. It allows the selection and grouping of (specific versions of) object classes into a conceptual model. Moreover, it provides some analytical functions, such as finding the object classes involved in some action of a particular object, or the object classes whose actions involve a specific attribute. For any conceptual model, a model browser lists the empirical models that have been derived from that model. It provides functions for the maintenance of this list. New empirical models can be added, existing ones can be modified or removed.

Where conceptual models are a collection of object classes, empirical models consist of a number of instances of these classes. New instances are added by selecting a class from the conceptual model, and defining the initial attribute values of the object of that class that is to be instantiated. A model browser also provides facilities to test an empirical model. It allows the decision maker to start, interrupt, and terminate model execution. At any given moment, the attribute values of the object instances in the model can be inspected in much the same way they were given their initial value.

Action logger

This support class is meant to facilitate the testing of empirical models. Action loggers can be "attached" to any action of an object class. Their primary purpose is to keep a record of the number of times an action is performed, and the average duration. An action logger can also be instructed to record more information, such as time stamps or specific parameter values.

Attribute monitor

This support class is very similar to the action logger class. An attribute monitor logs any change in the value an attribute of some specific object instance. Again, the objective is to collect and aggregate data. Usually this is achieved by calculating the mean and variance of a specific (numerical) attribute. But an attribute monitor could also record values in for example a time series.

\section{Statistical analyzer}

In many problem situations statistical analysis - both of sample data and of data obtained through simulation - is required for the construction and validation of an empirical model. This support class provides a wide range of statistical functions, such as regression analysis, analysis of variance, T-tests, least significant difference tests, etcetera.

\section{Task browser}

A task browser allows a decision maker to define and edit task structures. Basically, a task browser provides access to all task objects within the environment. In analogy to object classes, multiple definitions of the same task should be allowed, something which calls for proper version management. Tasks can be added or removed, but also edited by adding or removing subtasks and (coordinating) decisions, or by modifying its verbal description. The flow relation is defined by specifying for each task and coordinating decision its immediate successors.

It is not uncommon that two tasks are identified that have different names, but identical structures. The tasks in Figure 5-14 on page 103, for example, al have the same structure. To avoid redundancy, a task structure can be specified by an "alias": the name of some other task of which the structure has been defined. A task browser allows the decision maker to use aliases whenever this is appropriate.

A task browser also provides a number of analytical functions, such as determining a task hierarchy, pointing out concurrency, and detecting cycles in flow relations. It can also construct a decision structure of a task by taking all 
decisions of its leaf tasks together in one precedence graph. It can analyze this decision structure for cyclic dependencies, and check whether these cycles are present in the task structure, as should be the case.

Finally, a task browser allows the decision maker to test a task structure by "executing" it. For this purpose it includes the agenda mechanism described in Section 3.4. During execution, the task browser provides control functions identical to those of a model browser.

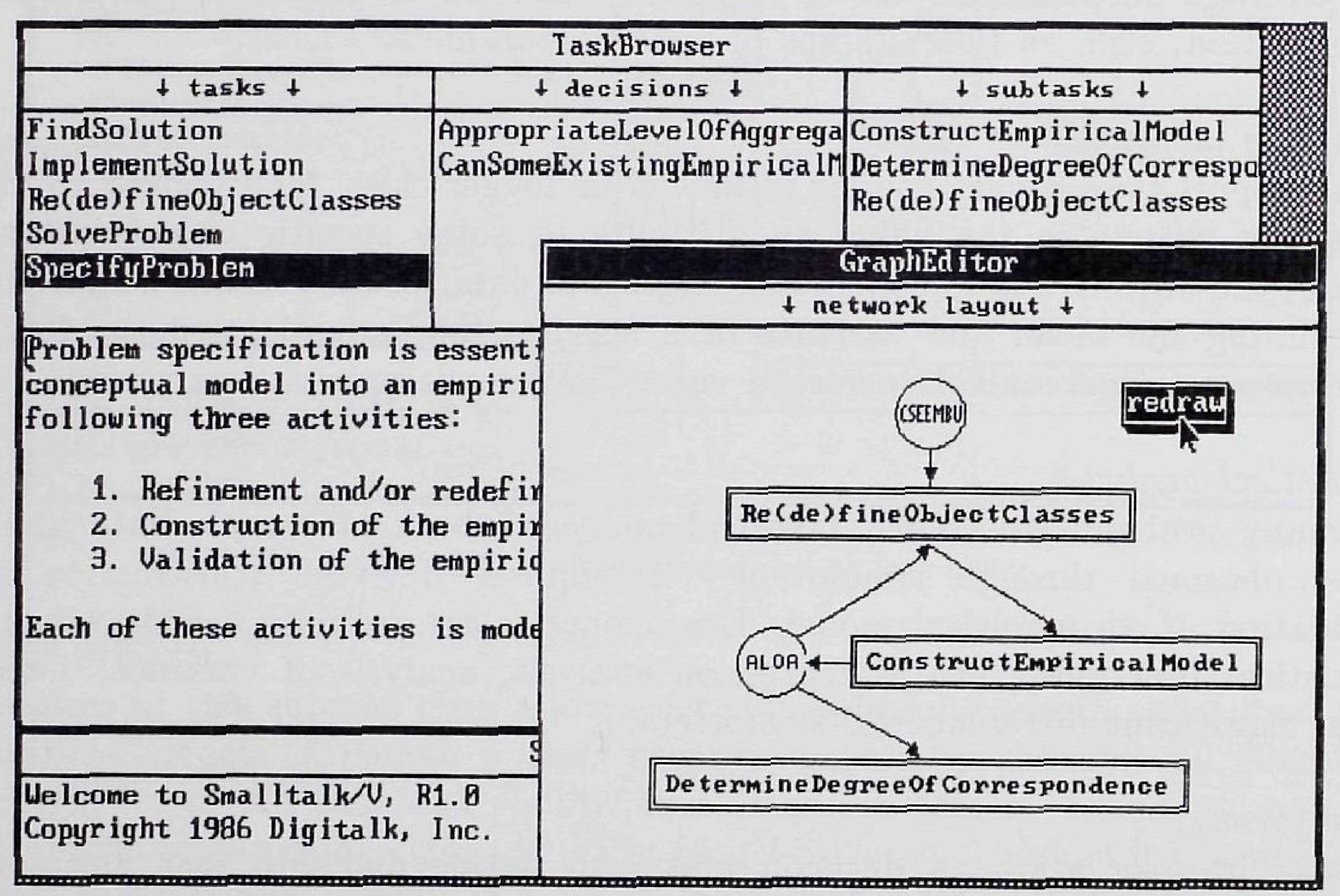

Fgure 4-21. A task browser and a graph editor

Figure 4-21 shows a task browser, implemented in Smalltalk as a four-pane window application. The first pane lists the tasks that exist within the PSSE. If a specific task is selected, the second pane lists its (coordinating) decisions, and the third pane its subtasks. Each of these three list panes has a menu that includes add, remove, and edit icon options. The first pane menu includes three additional options: alias, layout, and execute. The alias option activates a prompter that displays the current alias (if any), which may be modified. If the layout option is selected, a graph editor is activated on the structure of the selected task. If the execute option is chosen, the agenda mechanism is activated. The fourth pane of the task browser is a text pane that displays and allows modification of the description of the most recently selected item (being either a task, a decision, or a subtask).
Graph editor

In our PSSE graphs are an important means for visualization. Task structures mo be visualized as a graph: one in which the object classes figure as nodes, while the edges represent the interaction between objects (see for example Figure 5-3 on page 94 ).

A visual display of these graphs is desireable for purposes of communication and analysis. There are algorithms that lay out graphs with a minimum of crossing edges (Carpano 1980, Sugiyama et al. 1981; Rowe et al. 1987). Yet we found that these algorithms often produce a layout that is different from what one would have made by hand. This can be explained by the fact that a decision maker knows the exact meaning of a particular task, decision, or flow in a structure, and consequently places it in such a way that it reflects this meaning as much as possible. The graph editor in Figure 4-21 shows the task structure from Figure $4-8$ as it is laid out by the algorithm (note that the coordinating decisions are not labeled with their full name, but with only the first letters of the separate words). The difference between the two layouts is striking. Consequently, a graph editor must allow a decision maker to quickly lay out graphs by hand. The algorithm of course remains useful to generate a first layout.

Decision browser

A decision browser deals with decisions (defined in terms of information and support) in much the same way a task browser deals with tasks, which are defined in terms of subtasks and/or decisions. Its analytical functions are different, however. A decision browser does not generate a task hierarchy. Instead, it can list the influencing and affected information items of a decision, as well as a decision's immediate precursors and successors. Finally, if a particular decision can be "automated" in the sense that a decision rule can be defined that can replace a human decision maker, a decision browser allows the decision maker to associate this decision rule with that particular decision. When, during the "execution" of a task containing that decision, it is "put on the agenda", the decision rule is evaluated and the decision becomes passive again.

Figure 4-22 shows a decision browser as has been implemented in Smalltalk as a seven-pane window application. Four list panes providing access to all decisions that have been identified within the PSSE. When a particular decision has been selected from the left-most list pane, the other three list panes display (and allow modification of) the influencing and affected information, and the support component of that decision. The contents of the central text pane depend on the status of the two button panes at the bottom of window. If Description is activated, the text pane will hold the verbal description of the 
item that was most recently selected from one of the four list panes. If Making has been "pushed" (as is the case in Figure 4-22) the text pane holds the decision rule associated with the selected decision. This decision rule has the form of a Smalltalk context. We chose for this form to allow the user total freedom in specifying decision rules. The particular rule that is associated with the "Add parcel to parcel list?"-decision selected in Figure 4-22 states that if the expected delivery time of a some parcel exceeds its due time by more than $5 \%$ the decision maker is asked whether this should be allowed or not. Otherwise it is added to the parcel list immediately. Observe how this rule makes use not only of the decision's support component (in this case the support object VANSIMULATOR), but also of the Smalltalk class PROMPTER.

\begin{tabular}{|c|c|c|c|}
\hline \multicolumn{4}{|c|}{$\begin{array}{c}\text { DecisionBrowser } \\
\end{array}$} \\
\hline+ decisions + & $\downarrow$ influencing $\downarrow$ & $\downarrow$ affected $\downarrow$ & + support $t$ \\
\hline $\begin{array}{l}\text { AddDest inationToDes } \\
\text { AddParcelloParcelli: } \\
\text { ChooseBestRoute } \\
\text { SelectDriver } \\
\text { SelectUan }\end{array}$ & $\begin{array}{l}\text { Orders } \\
\text { Parcellist } \\
\text { Route } \\
\text { UanCapacity }\end{array}$ & Parcellist & VanSimulator \\
\hline \multicolumn{4}{|c|}{ 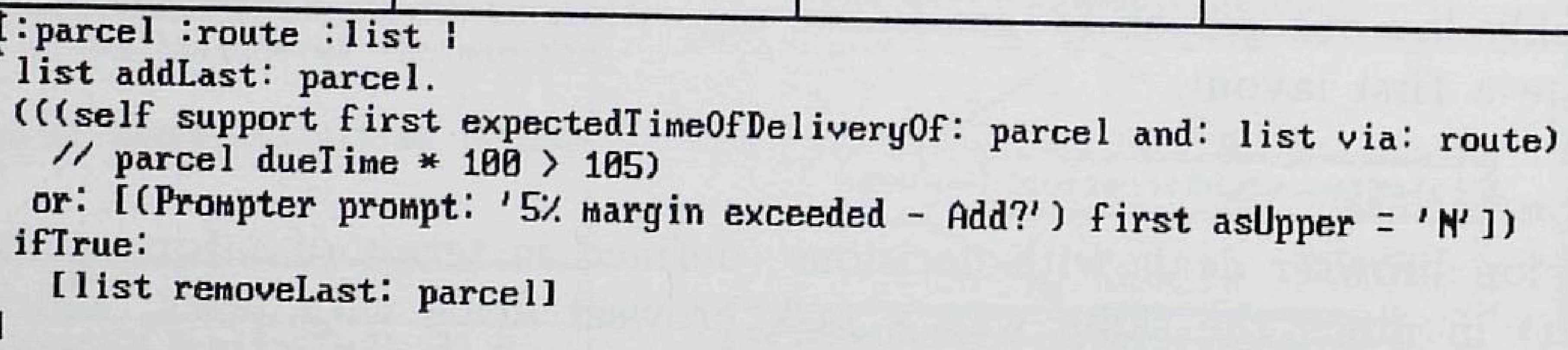 } \\
\hline \multirow{2}{*}{\multicolumn{2}{|c|}{1 desoription }} & \multirow{2}{*}{\multicolumn{2}{|c|}{1 making }} \\
\hline & $\begin{array}{ll}\text { Welcome to Snalltalk/U, R1.8 } & \text { System Iranscript } \\
\end{array}$ & & \\
\hline $\begin{array}{l}\text { Welcome to Snalltalk } \\
\text { Copyright } 1986 \text { Digit }\end{array}$ & $\begin{array}{l}\text { S/U, R1.8 } \\
\text { talk, Inc. }\end{array}$ & & \\
\hline
\end{tabular}

Figure 422. A decision browser showing a decision rule

Information browser

An information browser allows the decision maker to define information objects. Similar to a decision browser it provides functions for adding, editing, and deleting specific information items. The analytical functions are complementary to those of a decision browser: for each information item it can list the influenced and affecting decisions.

Instead of associating decision rules with decisions, the decision maker can define the "demon logic" for a particular information item by specifying what the contents (for example a specific value or value range) of that item must be in order to put an influenced decision "on the agenda".

Figure 4-23 shows an information browser, implemented in Smalltalk as a three-pane window application. The pane on the left lists the class names of information objects. Class definitions can be added or deleted, or edited, in which case the information browser automatically activates a class browser. If a particular class is selected, the pane on the right lists the names of all instances of that class. New instances can be added to the list, or existing instances can be removed or inspected, in which case the information browser activates an instance of the Smalltalk class INSPECTOR (Goldberg 1984, page 144148). The left pane of the inspector in Figure 4-23 lists the attributes of information class NETWORK, while the pane on the right shows the value of the selected attribute (in this case StrongConCom, which holds the list of strongly connected components of the network). The large text pane of the information browser holds either the comment string of the selected information class or - if some instance has been selected - the verbal description of that instance.

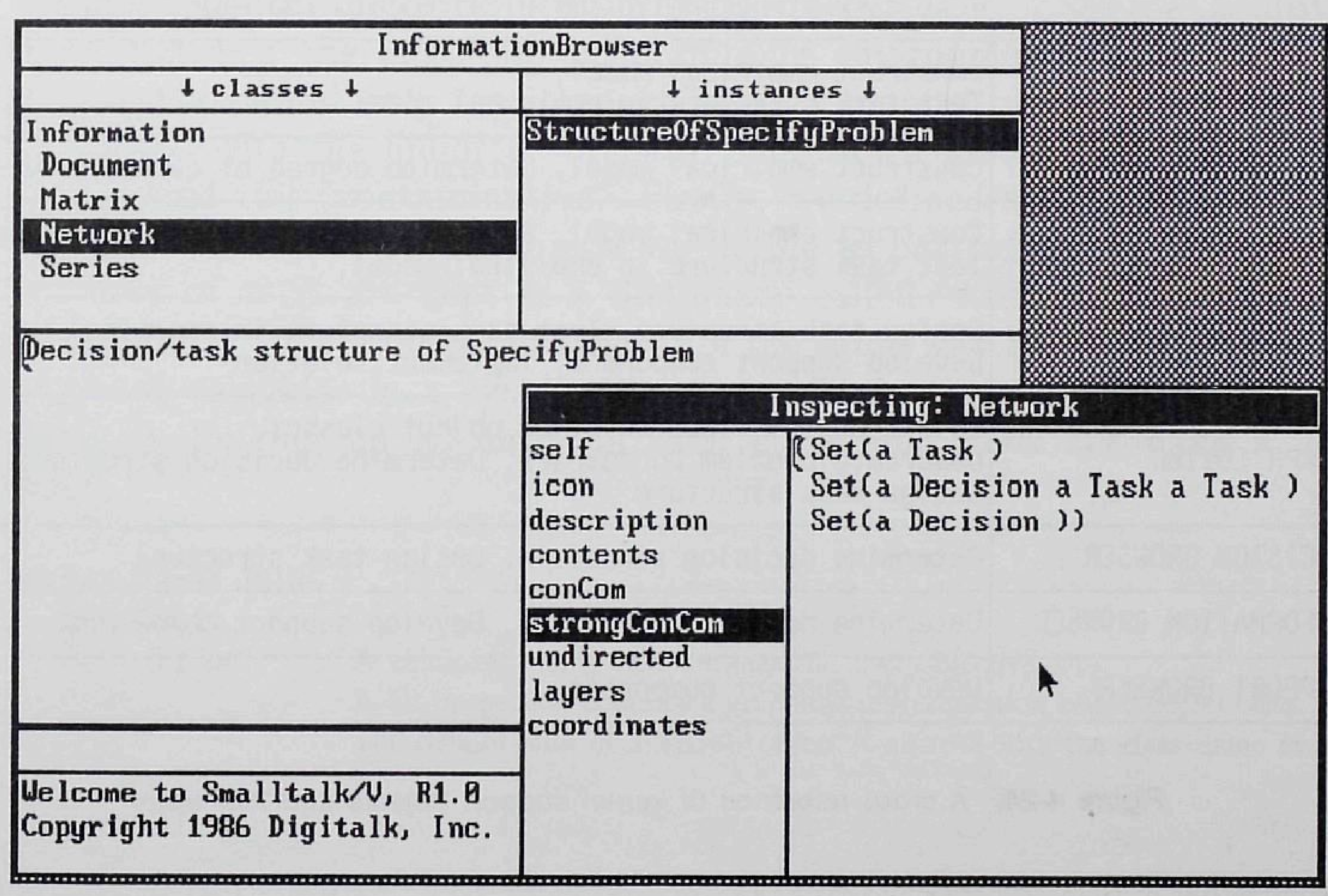

Figure 4-23. An information browser and an activated inspector

Support browser

A support browser gives a decision maker access to the full range of support classes (and for each class its instances) that exist within the environment. New 
support classes can be added only using a class browser, but the support browser allows the decision maker to instantiate and initialize support objects as he sees fit. This need not require substantial programming skills from the decision maker. Support classes can be defined in such a way that the novice is guided step by step through the object instantiation and initialization process.

\begin{tabular}{|l|l|}
\hline Kernel support object & Tasks \\
\hline \hline DOCUMENTATION BROWSER & All \\
\hline CLASS BROWSER & $\begin{array}{l}\text { Identify object class, Specify object class, } \\
\text { Relate object class to other object classes, } \\
\text { Re(de)fine object classes, Develop support component }\end{array}$ \\
\hline ICON EDITOR & Identify object class \\
\hline MODEL BROWSER & $\begin{array}{l}\text { Relate object class to other object classes, } \\
\text { Demarcate problem boundaries, Construct empirical model, } \\
\text { Determine decision structure, } \\
\text { Test task structure in empirical model, Implement solution }\end{array}$ \\
\hline ACTION LOGGER & $\begin{array}{l}\text { Construct empirical model, } \\
\text { Test task structure in empirical model }\end{array}$ \\
\hline ATTRIBUTE MONITOR & Construct empirical model, Determine degree of correspondence \\
\hline STATISTICAL ANALYZER & $\begin{array}{l}\text { Construct empirical model, Determine degree of correspondence, } \\
\text { Test task structure in empirical mode l }\end{array}$ \\
\hline TASK BROWSER & $\begin{array}{l}\text { Design task structure, Test task structure in empirical model, } \\
\text { Develop support component, Implement solution }\end{array}$ \\
\hline GRAPH EDITOR & $\begin{array}{l}\text { Relate object class to other object classes, } \\
\text { Demarcate problem boundaries, Determine decis ion structure, } \\
\text { Design task structure }\end{array}$ \\
\hline DECISION BROWSER & Determine decision structure, Design task structure \\
\hline INFORMATION BROWSER & Determine decision structure, Develop support component \\
\hline SUPPORT BROWSER & Develop support component \\
\hline
\end{tabular}

Fgure 424. A cross reference of kernel support objects and leaf tasks

\subsection{IMPLEMENTATION ISSUES}

To test our method of task analysis we conducted a number of case studies, which will be discussed in Chapter 5, 6, and 7 . In parallel to these case studies, which provided the necessary experience, we developed a prototype PSSE using Smalltalk/V ${ }^{\mathrm{TM}}$ (Digitalk 1986) on a PC-AT. In the previous section we showed a number of Smalltalk screens to visualize some of the kernel support objects. In this section we address a number of implementation issues in more detail.

Representing tasks and decisions as objects

Not only is "Smalltalk" the name of a programming language, it also refers to the interactive programming environment described by Goldberg and Robson (1983). This environment includes a large collection of "basic" class definitions that define the primitive and more complex data structures and their operations, the compiler, and the building blocks of the graphical user interface. A Smalltalk programmer can add his "own" class definitions to this set, but he also may modify existing classes. This makes the Smalltalk programming environment a very flexible tool indeed.

When implementing our prototype we decided to preserve a distinct boundary between the Smalltalk programming environment and our PSSE. A decision maker should only "see through" kernel support objects. He should not be confronted with the hundreds of basic Smalltalk classes. We employed two means to avoid this "contamination". Firstly, we defined the class THING, which serves as the superclass of all objects we define as part of the PSSE. Secondly, all instances of THING are kept in a dictionary called "WorLD" (note that we use small capitals to denote object instances) in the same way Smalltalk keeps track of its global variables in a system dictionary. Since the name of a thing can be found by looking up its entry in WorLD, class THING does not have a name attribute.

\section{attributes \\ Description}

object class THING

Icon

A character string that outlines the task objective.

A bit image that contains a graphical representation of the object class.

The default icon of a THING object is an oval with the class name in

The "building blocks" used in task analysis are implemented using but four object classes: TASK, DECISION, INFORMATION and SUPPORT. All four are subclasses of class THING, and therefore inherit the Description and Icon attributes. 


\section{object class TASK \\ superclass THING \\ attributes \\ Alias}

If the task's structure is identical to that of a previously defined task, Alias contains a pointer to that task

Decisions The collection of decision objects (either coordinating or leaf) that are part of this task.

Subtasks The collection of subtasks of this task (the next layer in the task hierarchy).

Successors The collection of objects that are put on the agenda when this task terminates.

Structure An instance of class NETWORK, representing the flow diagram (see page 47) of this task. We do not give the complete definition of NETWORK here; its actions include methods for graphical display, and for analysis: the results of precedence analysis.
the and comparing the flows with

Since leaf decisions and coordinating decisions have most of their attributes in common, we define an object class DECISION as follows:

object class COORDINATING DECISION

superclass DECISION

attributes

Conditional successors A table indicating which task and decision objects should be

]

object class DECISION

[ attributes

Influencing info A list of instances of (subclasses of) INFORMATION that should be

Inf luencing info A list of instances of (subclasses of) INFORMATION
available for inspection when this decision is made.

Affected info A list of instances of (subclasses of) INFORMATION whose contents can

be modified when this decision is made.

A list of instances of subclasses of SUPPORT that can be activated

Rule when this decision is made.

Wolds the a compiled version of a Smalltalk context that specifies how the support object(s) of the decision should A decision rule. info, Affected info, or Support!

The object classes INFORMATION and SUPPORT have a much larger number of subclasses. Information objects are very diverse: documents, images, facts, rules, time series, matrices - even NETWORK is an information object. There will be a large variety of support objects as well. For example, each of the kernel support classes mentioned in the previous section has been implemented as a subclass of SUPPORT.
Using Smalltalk classes as kernel support objects

When implementing the document browser class we made use of the fact that the Smalltalk coding conventions (the templates for class and method definitions) include a comment string. Together with the descriptions of THING instances, these comment strings constitute the "document base" of the PSSE. The document browser prompts for a search string and then scans the dictionary WORLD for THING instances with a description that contains this string. It then scans the Smalltalk system dictionary for "matching" class definitions.

The class browser that is part of the Smalltalk programming environment provides all the functions necessary to create, modify, or delete object class definitions. In order to preserve the boundary between the Smalltalk programming environment and our prototype PSSE we made a copy of Smalltalk's CLASS HIERARCHY BROWSER class (Goldberg 1984, page 192-193), and added it as a subclass of SUPPORT. Subsequently, we made sure that the only class definitions that could be accessed through this support object were THING and its subclasses.

Recall that a conceptual model consists of object classes. These classes can be manipulated using an instance of CLASS BROWSER. Using the senders and receivers options on a class browser's message list pane, valuable information concerning the interaction between different object classes can be obtained. An empirical model is a collection of object instances. Within our prototype PSSE these objects are stored in a dictionary. We did not define a special support class that allows manipulation of empirical models, but use Smalltalk's DICTIONARY INSPECTOR (Goldberg 1984, page 156) instead. Using this inspector, objects can be added to the model, and their attribute values can be changed.

Tracking model behavior

The obvious way to implement an action logger would be to modify the Smalltalk primitive send:to: in such a way that any message sent to an object with THING as its superclass is recorded automatically. However, we rejected this approach as being too complicated. We did not have access to the source code of the Smalltalk system primitives, and - what is more - we figured that recoding part of a prototyping tool when building a prototype just does not fit in the prototyping philosophy. We therefore decided to implement action loggers by defining an extra attribute Logger of class THING to refer to an instance of class ACTION LOGGER, and by adding the statement "Logger update: <action names" at the beginning of each action method that is to be logged. Depending on the logger's settings it will keep simple tallies, or maintain a more detailed record.

When implementing the ATTRIBUTE MONITOR class we took a similar approach. First, we made a rule saying that the attributes of an object may only change as the result of some action. Provided this rule is observed, we can implement attribute monitors in exactly the same way as we implemented action loggers: We define an extra attribute of class THING to refer to an instance of ATTRIBUTE 
MONITOR, and we add the statement "Monitor check: <attribute names" at the end of each action that may affect the value of the attribute that is to be logged.

We must emphasize that our implementation in Smalltalk/V on a PC-AT cannot be called a truly operational PSSE prototype. Ironically, the available technology proves to be the constraining factor, neatly counterpointing the remark we made at the very beginning of this thesis. Even when using but a small task structure, like for instance the structure of "Solve problem", which consists of less than 100 objects, the system's virtual memory management system tends to crash at unpredictable moments.

Mainly for this reason we decided to develop a second prototype, this time using an imperative programming language. The functionality of this second prototype significantly differs from that of the PSSE described in the previous section. The focus has shifted from testing the feasibility of a PSSE as a whole to developing supporting software for our task analysis technique. The reason for this change in emphasis is the fact that the Smalltalk prototype has proved to be quite adequate for the construction of conceptual and empirical models. Moreover, it sufficiently demonstrates that construction and integration of support objects for the subtasks of "Solve problem" is feasible. The Smalltalk prototype falls short mainly on one point: supporting task analysis of larger problems. The primary function of our second prototype is to compensate for this shortcoming.

Consequently, the software in directory $\backslash$ TASK on the diskette that came with this thesis does not constitute a prototype PSSE, but rather a "task analysis support kit". It has been developed in Turbo Pascal ${ }^{\text {TM }}$ (Borland 1987), and requires an MS-DOS ${ }^{\text {TM }}$ machine with $640 \mathrm{~K}$ of internal memory, a hard disk, an EGA-compatible graphics screen, and a Microsoft ${ }^{\mathrm{TM}}$-compatible mouse (mouse driver version number 6.11 or higher). We do not describe the exact functionality of the task analysis support kit. Instead, we suggest that the reader takes a look at the software and its on-line information facility.

\subsection{CONCLUSIONS}

The material presented in this chapter allows us to draw two important conclusions:

1. the task analysis technique described in Section 3.4 can be applied successfully to a problem at a high level of abstraction, and leads to an adequate specification of support requirements;
2. it is feasible to develop a PSSE with the functional requirements specified in Section 4.3.

To this we add the remark that the task structure from Section 4.2 is itself a major part of the PSSE, since it guides a decision maker through the entire process of problem solving. The twelve classes of kernel support objects alone do not provide this guidance. Figure 4-24 clearly indicates that these support objects are not "task-specific" in the sense that they support one problem-solving stage (conceptualization, specification, solution finding, or implementation) in particular. 


\section{INDIVIDUAL PROBLEM SOLVING SUPPORT}

\section{THE CASE OF SHIFTWORK PLANNING}

Let every hour be in its place, firm fixed nor loosely shift, and well enjoy the vacant space as though a birthday gift.
- Lewis Carroll

\subsection{INTRODUCTION}

Our first case study focuses on individual problem solving, meaning that we investigate the problem-solving processes of a single decision maker. The specific problem we shall be looking at lies in the area of personnel planning. Personnel planning is an activity that is performed within a variety of organizations, and problems with personnel planning are very diverse in nature.

In this chapter we consider one specific subclass of planning problems: the planning of shiftwork. Shiftwork planning is done in many organizations. It becomes necessary whenever an organization's production hours, which may be up to 168 hours per week, exceed its employees' working hours, which will rarely exceed 48 hours per week. Shiftwork is a form of work organization in which the organization's production hours are partitioned in "shifts", and two or more groups of workers are assigned to these shifts. Thus, different groups of workers operate in succession.

These groups are referred to as "crews" or "teams". We make the following distinction between crew and team. A crew is a group of workers who are assigned to a particular production unit. A team is a group of workers who can be assigned to that production unit. Thus, the team for a production unit may consist of for example 16 workers, even though a crew consists of only 12 . There are several reasons for rostering with a surplus of workers, as we shall indicate later on

To achieve a balanced distribution of work among crews, a crewing system is used. A typical example is the four-crew system depicted by Figure 5-1. This crewing system combines non-stop operation (168 production hours) with an average of 42 working hours by assigning four crews to three 8-hour shifts: the Morning, Afternoon and Night shift. A dash indicates a rest period.

Crewing systems always are cyclic. Cycle lengths may vary widely, depending on the number of crews and shifts. As a rule, each crew follows the same cycle. This guarantees a balanced distribution of shifts over crews. Crewing systems must also meet specific conditions like a minimal and maximal number of consecutive work shifts, an adequate rest period after each "block" of consecutive work shifts, and an equal distribution of "inconvenient" shifts, such as night shifts and shifts during the weekend. Note that crewing systems are 
characterized by a fixed production hours/working hours ratio, equal to the number of crews.

$$
\begin{array}{|cl|}
\hline \text { Crew } & \text { Shift cycle } \\
\text { A } & \text { AAAAAAA--MMMMMMM--NNNNNNN--- } \\
\text { B } & \text {--MMMMMMM--NNNNNNN---AAAAAAA } \\
\text { C } & \text { MM--NNNNNNN---AAAAAA--MMMMM } \\
\text { D } & \text { NNNN---AAAAAA--MMMMMMM--NNN } \\
\hline
\end{array}
$$

Fgure 5-1. 168 hour four-crew system with three 8-hour shifts

The basic principle of shiftwork is that the members of a team all have the same roster, which means that crews and teams are identical. But, like we have said earlier, there are reasons why this principle is not absolute.

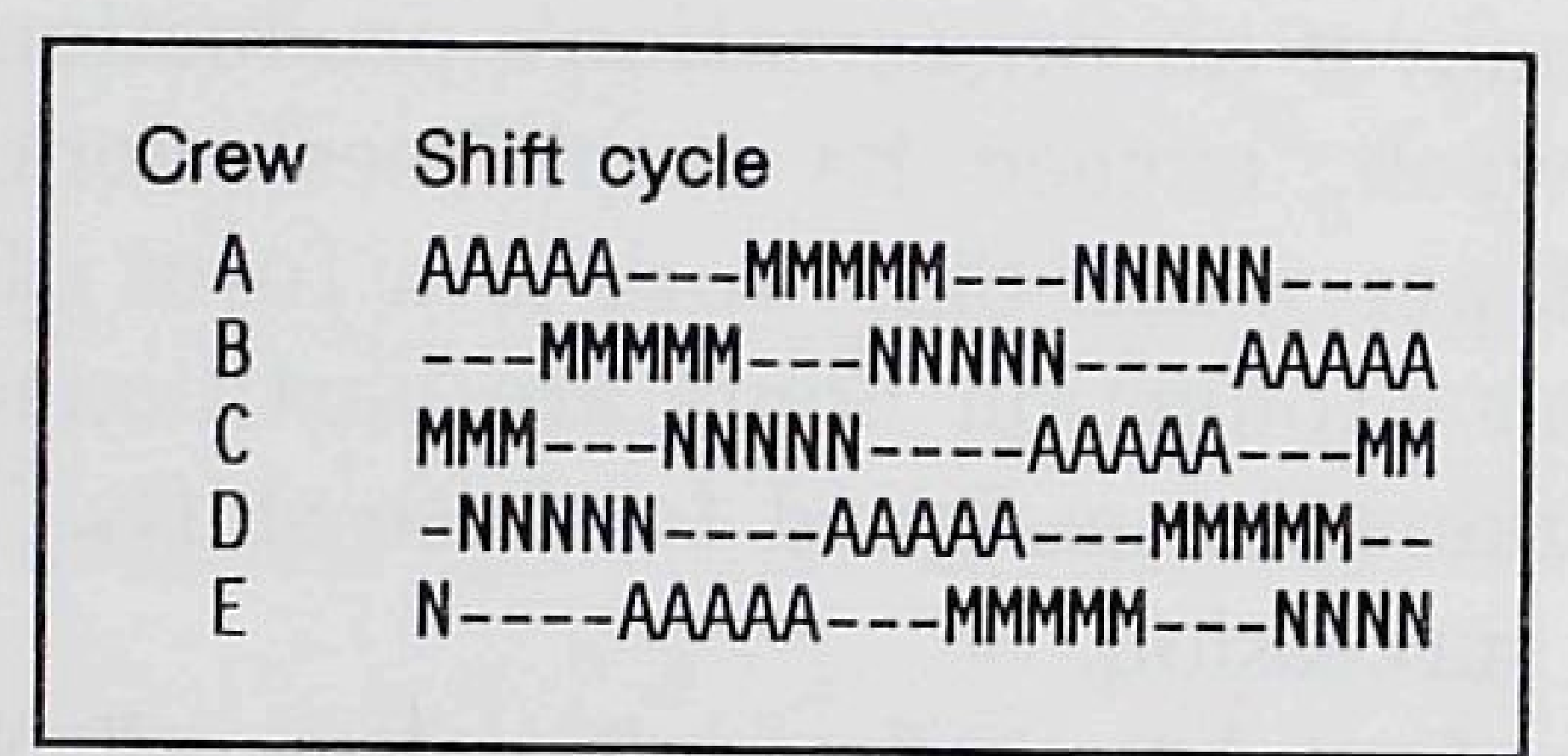

Figure 5-2. 168 hour five-crew system with three 8-hour shifts

The first reason is the recent trend of decreasing working hours while maintaining or even increasing production hours. This has complicated shiftwork planning. For a non-stop producing organization to decrease its working hours from 42 to 40,38 or even less is not an easy matter. If it changes from a four crew to a five-crew system like the one in Figure 5-2, the working hours would be reduced to $168 / 5=33.6$ hours per week. Since this decrease is considered too large, most organizations resort to one or several of the following instruments:

1. Maintain the original four-crew system, but give each worker 13 shifts off over a one-year period. This adds up to 104 hours per year, effectively reducing the working hours to 40 . This obviously leads to severe planning problems, as these extra days off should be spread out evenly over time, and the favorable situation of such a day falling in a weekend should occur equally often for each individual. Meanwhile, the organization's manning requirements have to be met at all times.
2. Use the original four-crew system in combination with the five-crew system. Thus, an average of approximately 38 working hours can be realized by operating in a four-crew system for 6 months, and in a five-crew system the rest of the year.

3. Use the five-crew system as a basis for individual rosters and increase the average working hours to the desired level by assigning shifts to each worker. This leads to similar problems as the first instrument does.

Another factor that makes the planning of shiftwork more complicated is the growing acceptance of part-time work. This can be very advantageous for an organization, providing a more flexible working force. But it is extremely hard on the personnel manager, since it requires individual treatment of each worker. Moreover, it complicates the judgement of the "fairness" of the allotment of the extra days off.

A third factor is that allowing for individual rosters provokes demands from the personnel. Some workers may prefer certain days over others, while there may be specific days of the week they cannot work. Accommodating for such individual preferences may become a necessity under pressure of the unions.

In all, we based our decision to take shiftwork planning as the subject of a case study on the following considerations:

\section{Relevance}

We were looking for a problem of present interest, and preferably of a general nature, meaning that similar problem situations should occur within a substantive number of organizations. The reduction of working hours, the growing "supply" of part-time workers and the need for organizations to increase the flexibility of their work force have significantly complicated shiftwork planning. The present interest is clearly indicated by for example Singer and Berger (1986), Wallace (1986), Dawson (1987), Jacob (1988), and Ouwehand (1989).

Demarcation

The problem had to be "contained" in the sense that the impact of other problem-solving processes in the decision maker's environment was only secondary. Otherwise it could not be considered as individual problem solving. Although both the production department and the unions play an important role in setting conditions, the actual planning is performed by the personnel manager only. 
Complexity

The problem situation had to be complex in the sense that it surpassed the cognitive ability of the decision maker. His problem-solving process should be heuristic in nature. Presently, rostering is done mainly by hand, using a satisficing approach. The crewing system determines the basic roster for crews, and personnel managers rely on their experience in tailoring this roster to the manning requirements of specific production units. Checking whether the individual rosters are fair and at the same time conform to the conditions set forth in the common labor agreement (CLA) is a time-consuming activity, since numerous tallies (number of shifts, weekends and working hours, compensation for inconvenience) must be kept by hand.

\section{Structuredness}

The specific problem situation had to be ill-structured in the sense that little or no formalization of the problem-solving process was to have taken place so far The decision maker should not previously have used an explicit strategy. As a rule, personnel managers do not receive special training in rostering, but get the "knack" through apprenticeship. The rostering process usually is one of trial and error.

\section{Field test}

The problem situation had to be "real-life" in the sense that organizations could be found within which proposed solutions and supporting technology could be tested using company data. Moreover, the problem had to be of a recurring nature, so that several experiments could be conducted in a two-year period. With the General Industrial Employers' Association (AW-V 1987) as an intermediary, several large organizations in which shiftwork planning was performed on a regular basis were found willing to participate in our research as a testing ground.

These considerations lead us to believe that shiftwork planning constitutes a suitable case study on individual problem solving. In brief, a personnel planner faces the problem to create individual rosters for all personnel that at the same time are fair, meet the organization's manning requirements while minimizing the personnel surplus, and accommodate for individual preferences.

In this chapter we shall analyze this problem in depth, using the problemsolving approach we described in Chapter 3 . In Section 5.2 and 5.3 we describe the activities of problem conceptualization and specification, respectively, while in Section 5.4 we show how task analysis is used during the solution finding activity. In Section 5.5 we describe how the personnel manager's tasks can be supported by means of information technology, and how this support has eventually been implemented. We end this chapter in Section 5.6 by summarizing this case study and presenting our main conclusions.

\subsection{CONCEPTUALIZATION}

Following the approach described in Chapter 3, we start out with identifying the object classes in terms of which shiftwork planning can be described. We define object classes in terms of attributes and actions. In this particular problem situation, most objects are "passive" in the sense that they do not perform actions themselves. Rather, they are affected by actions of other objects.

The decision maker we are looking at is the personnel manager of a firm that uses shiftwork as its work organization. We therefore immediately identify our first object class: PERSONNEL MANAGER. We know that there will be but one instance of this class. Since PERSONNEL MANAGER is likely to be the most complex object class, we save it for last. Next, we identify the object classes that correspond to "physical" objects: PRODUCTION UNIT, WORKER and TEAM. These classes can be defined as follows:

\section{attributes \\ Working hours \\ Manning requirements \\ Crewing system \\ Inconvenience \\ ] \\ object class TEAM \\ [ attributes \\ crew}

object class PRODUCTION UNIT

\section{object class WORKER}

\section{attributes}

Name

Registration number

Skills

Contractual hours

actions

acquire skill

change contractual hour

be absent

signal preferred and disliked shifts
For each shift on each day of the week the duration of that shif.

each function for each day of the week the number of workers that must fulfil that function. unit.

For each shift on each day of the week the relativ inconvenience of this shift, expressed as a percentage of th working hours.

The code (for example $A, B, C$ or $D$ in a four-crew system) of the crew that is to be selected from this team. The workers that are on this team.

Skills determine the functions in which this worker can

The ner week this worker wishes to work.
The crewing system that will be used for this production operate in a crew. 
The attributes of production units are determined by the firm's production department. We identify PRODUCTION DEPARTMENT as an object class, but since we are not interested in production planning here (we save that for Chapter 7), we do not define it in as much detail as we will define PERSONNEL MANAGER.

\section{object class PRODUCTION DEPARTMENT}

\section{[ actions}

change the working hours of a production unit

change the manning requirements of a production unit

We must take into account that workers are unionized. The unions play an important role in setting labor conditions. We therefore identify UNION and LABOR CONDITION as an object class:

\section{object class UNION \\ [ actions}

\section{object class LABOR CONDITION}

\section{attributes}

Condition

Weight

Specifies a constraint, such as "no more than five consecutive night shifts".

]

A factor indicating the importance of this condition relative to that of other conditions.

The definitions of the object classes we identified so far make reference to objects such as crewing system, shift, function, and labor condition. For these objects we define the following object classes:

\section{object class CREWING SYSTEM \\ attributes}

\section{Name}

Number of crews

Cycle length

]

The number of days (shifts) in one cycle.

object class SHIFT

[ attributes

Code

$$
\text { Description }
$$

]
One cycle for each crew.

Usually a one-letter code.

The name of the shift, and for example the start and end time: "Night shift, $10 \mathrm{pm}-6 \mathrm{am}$ ".

\section{object class FUNCTION}

[ attributes

Code

For example a two-digit number.

Description

The skills that are required for this function.

Planning shiftwork means making rosters for teams of workers for a certain period of time. We therefore define the object class ROSTER PERIOD:

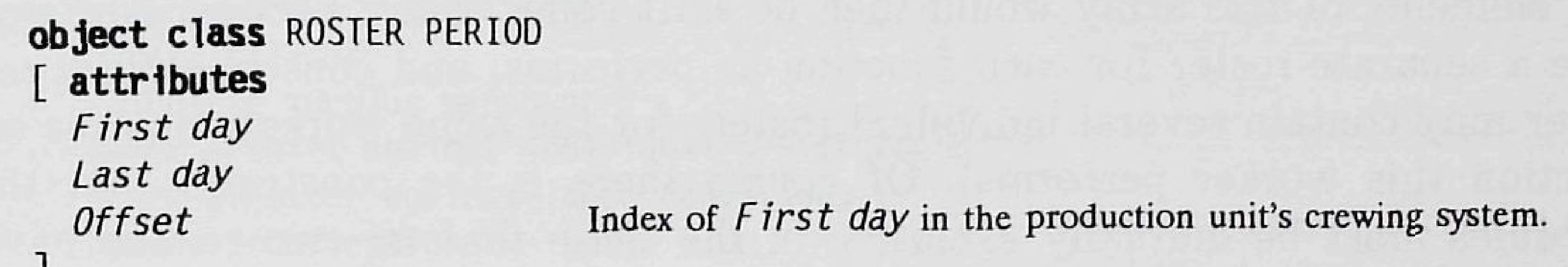

By specifying the offset attribute of a roster period, the personnel manager determines where in their shift cycle each of the teams will be on the first day of the roster period. For example, if a production unit uses the five-crew system from Figure 5-2, and if the roster period starts on Sunday, January $1^{\text {st }}, 1989$, the value of offset should be set to 12 to establish that crew A will start to work on monday morning, do four morning shifts, take two days off, start with the first of three night shifts on saturday, etcetera. In other words, the choice of an offset determines how the shift cycles for each crew in the crewing system are mapped onto the planning period. This "mapping" results in a so-called "crew roster" for each crew. Even if crews and teams are not identical, we still speak of a crew roster, since this roster is the basis for each individual roster

The roster period, the crew roster, the team of workers, and their individual rosters together constitute a "team roster", which we identify as an object class:

\section{object class TEAM ROSTER \\ [ attributes \\ Period \\ Team \\ Crew roster \\ ]}


As a rule, a personnel manager will be working on one team roster at the time, since there is no overlap between teams. Situations in which one or more workers from team $x$ temporarily replace workers in team $y$ are relatively uncommon.

More often, a worker will be assigned to different functions within the same team because he has the required skills. We shall consider such a situation in the next section. For now, the most important consequence is that a worker's individual schedule must indicate both the shift and the function in which he has to work. One way of dealing with this issue is to consider an individual roster as an array with time as its first dimension and function as its second. The elements of this array would then be shift codes. Thus, each worker would have a separate roster for each function he performs, and consequently a team roster may contain several individual rosters for the same worker (one for each function this worker performs). Of course there is the constraint that these schedules must be mutually exclusive in the sense that no two rosters have shift on the same day.

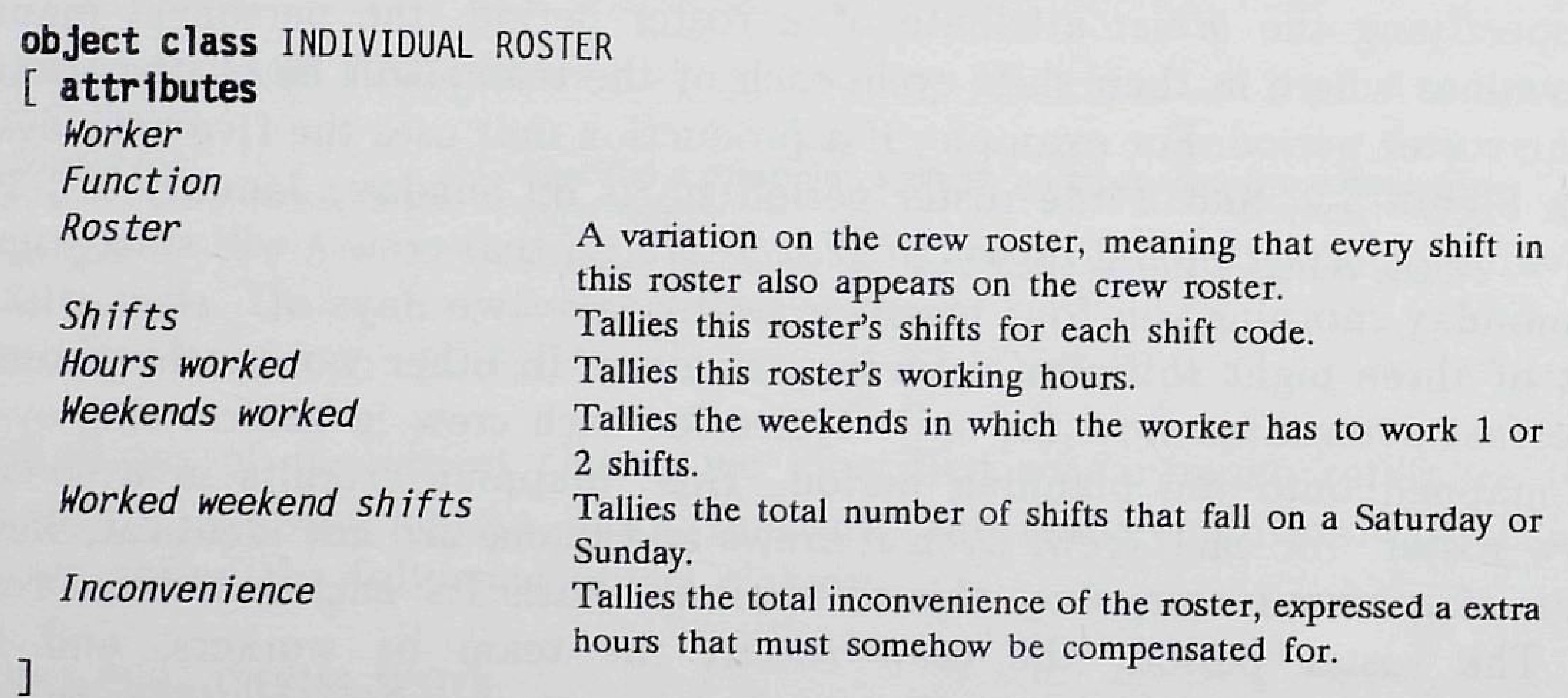

The tallies of an individual roster can be computed from its Roster and the attributes Working hours and Inconvenience of the production unit for which the rosters are made.

Having defined all object classes we find necessary to model the problem situation, we can return to our PERSONNEL MANAGER object class. As there will be exactly one instance of this class, we do not bother to identify attributes such as name, etcetera. Rather, we are interested in the actions this personnel manager performs.

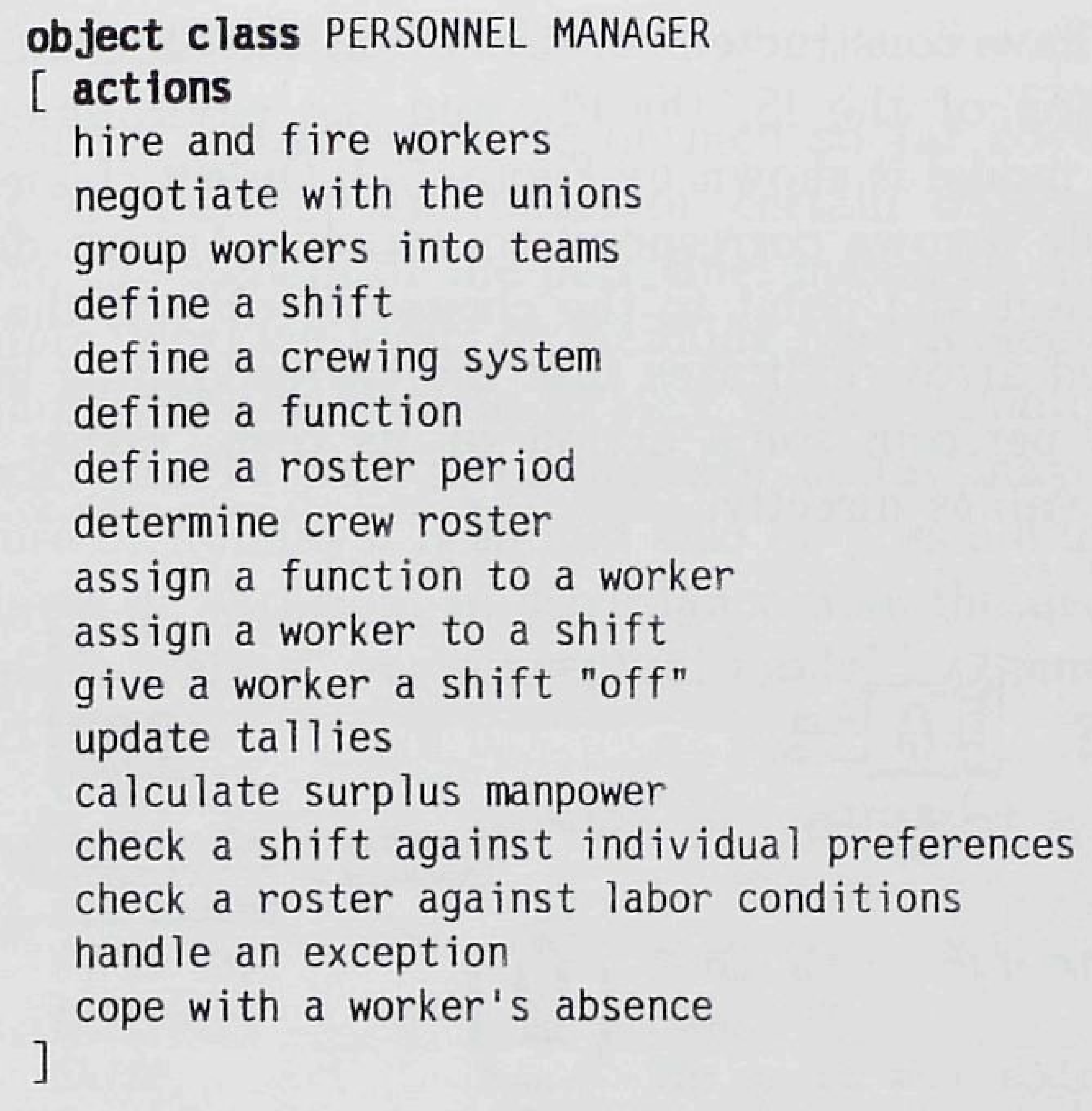

Now that we have identified and specified the object classes, we must determine the boundaries of the problem situation. The information paradigm is a useful instrument here. We view a problem situation as an IS/RS-combination. Since we wish to support the personnel manager in his problem-solving process, the obvious choice is to consider the personnel planner as the IS-component of this IS/RS-combination. But since we are interested only in the personnel manager's planning problems, we will not consider his first two actions.

Having defined the IS, the demarcation rule will help us in delineating the RS and the environment. Since the personnel manager has no direct control over the attributes of production units, the unions, the labor conditions, the absence of workers, or their personal preferences, all instances of these object classes belong to the IS/RS-combination's environment. This means that none of these objects may perform actions that directly affect objects that are part of the RS.

Obviously, the personnel manager can define shifts, functions, crewing systems, roster periods, and team and individual rosters, meaning that these objects belong to the RS. But what about workers, and teams of workers? The personnel planner has control over a worker's registration number, but not over his skill level or his contractual hours. We therefore consider workers as part of the environment, rather than of the RS. When we look at the definition of TEAM we see that, although he may not have control over individual workers, the personnel planner has control over both TEAM attributes. He determines who will be on a specific team, and the shift cycle that team will follow. This qualifies teams as part of the RS. 
Thus, the conceptual model we have constructed consists of all the object classe we defined, and our demarcation of the IS, the RS, and the environment. A graphical representation of this model is shown by Figure 5-3. Object classes are represented by their icons, while arrows correspond to actions. Arrows depart from the class of the acting object and point to the classes of objects that are affected by the action. A dashed arrow indicates that the corresponding action induces the affected object to perform some action of its own, rather than changing some of its attribute values directly.

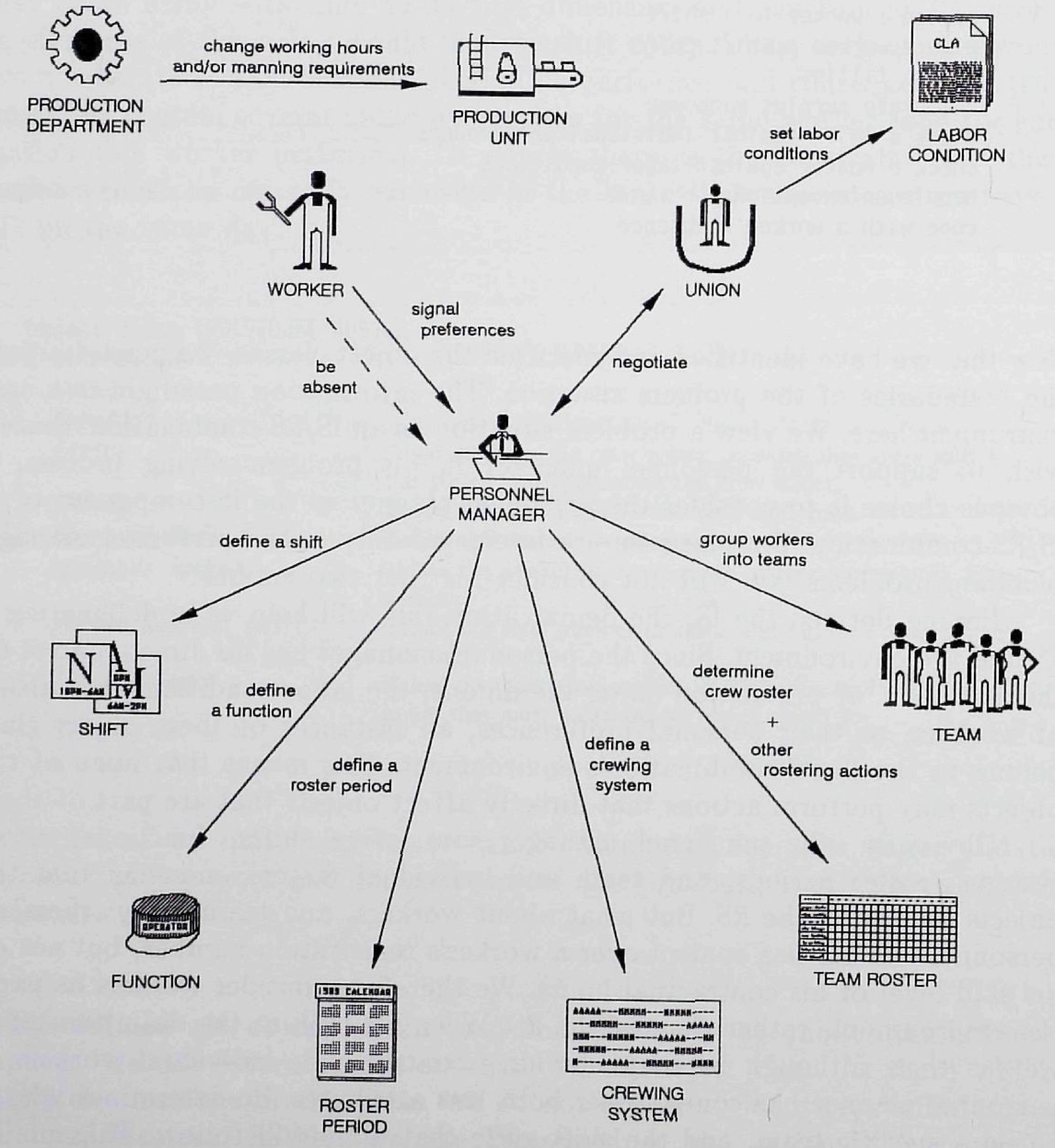

Flgure 5-3. A graphical representation of the shiftwork planning conceptual model

\subsection{SPECIFICATION}

The object classes we have defined so far cover most aspects of the problem situation. But the attributes of certain objects, in particular those that are beyond the control of the personnel manager, may show some irregularities. For example, working hours or manning requirements may be increased or decreased for short periods of time to stay within inventory limits, or shifts that usually do not deserve any inconvenience bonus may be considered as inconvenient because of holidays. It should also be possible to model absence of workers due to illness or vacation. In accordance with the decision structure in Figure 4-9 we therefore "refine" our conceptual model by identifying the following "artificial" object classes:

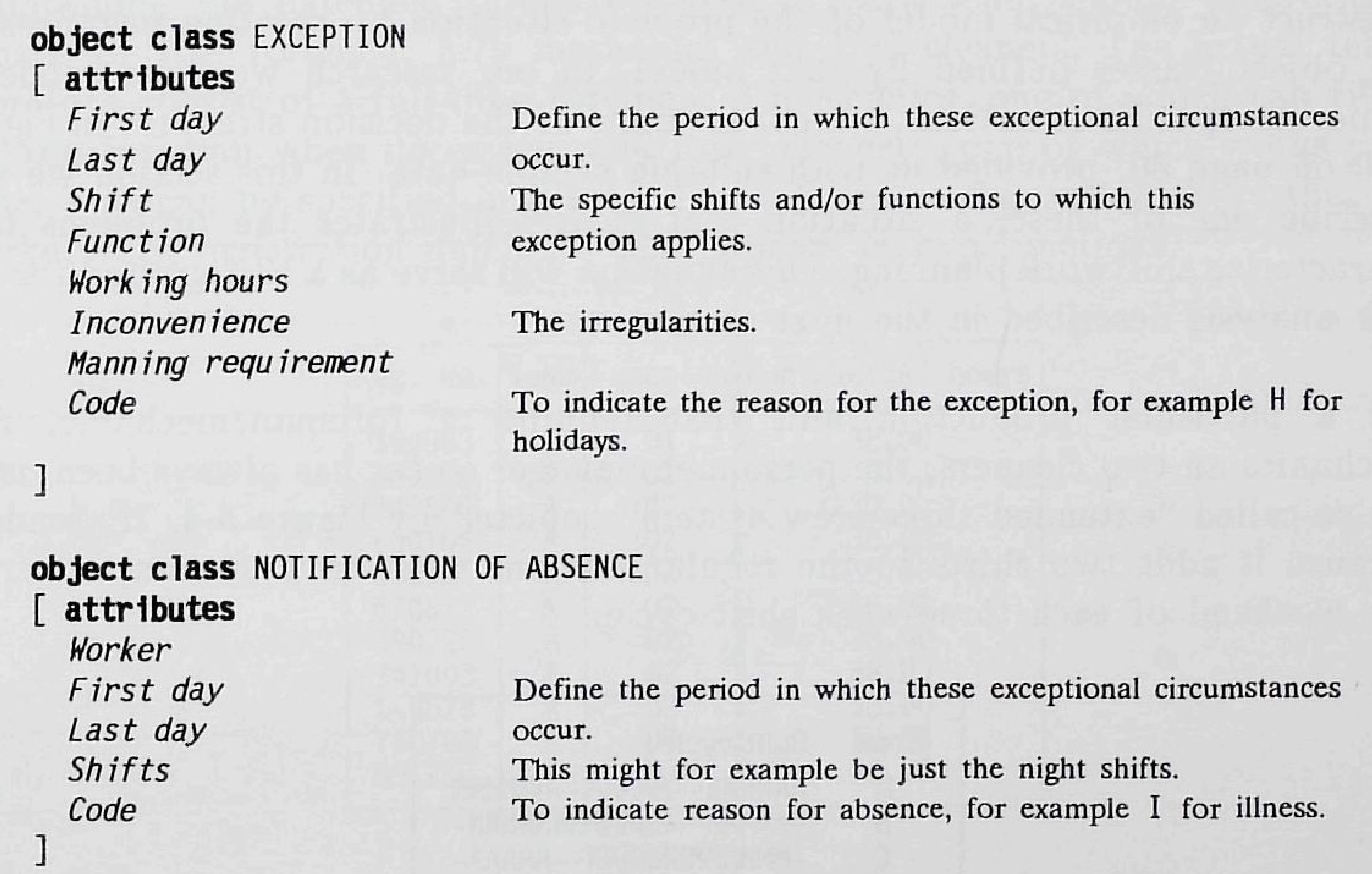

Finally, we need an object class to model the fact that workers may have particular preferences with respect to the shifts they do and do not wish to work (although there will doubtlessly be limits to the consideration of the personnel manager):

object class NOTIFICATION OF INDIVIDUAL PREFERENCE
[ attributes
Worker
Favored shifts
Disliked shifts


These object classes are artifacts because there is no direct correspondence with objects in reality. The production schedule for a production unit, for example, usually does not distinguish between "normal" and "exceptional" production hours and manning requirements. We, however, do identify a production "norm" and deviations from that norm as separate object classes to facilitate the modeling (and quick data entry and modification) of a production schedule in our empirical model. Similarly, notifications of absence and individua preferences are artificial constructs to model the interaction - usually face-toface or by telephone - between workers and the personnel manager. Note that each of the three new classes is part of the IS/RS-combination's environment.

Having thus extended our conceptual model of shiftwork planning, we can construct an empirical model of the problem situation by creating instances of the object classes defined by that model. In our research we have modeled numerous specific situations, which, in terms of the decision structure in Figure $4-10$ on page 60 , provided us with suitable sample data. In this section we will describe one of these, a situation that clearly illustrates the problems that characterize shiftwork planning. This situation will serve as a background for the task analysis described in the next section.

For a particular production unit that requires a foreman/mechanic, five mechanics an two cleaners, the personnel manager so far has always been using the so-called "extended three-crew system" depicted by Figure 5-4. "Extended" because it adds two shifts to the regular 40-hour three-crew system, covering one weekend of each three-week shift cycle.

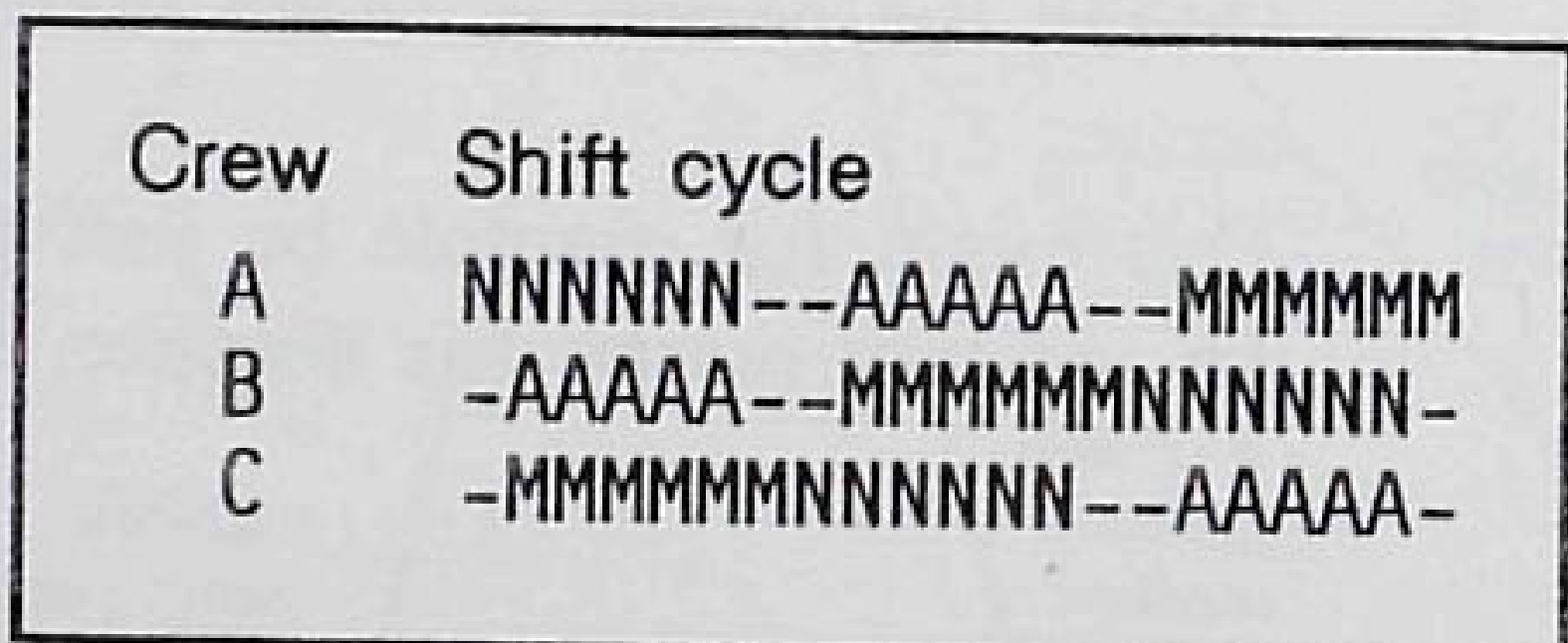

Fgure 5-4. The extended three-crew system

The Saturday morning crew shuts down the installation, while the Sunday night crew performs the start-up. Thus, the extended three-crew system has the advantage that a full 120 hours of non-stop production is guaranteed.

For this particular production unit both working hours and manning requirement of the extra shifts are less than those of the other shifts: 6-hour instead of 8-hour shifts, and only half-size crews (see Figure 5-5). This means that the average working hours are $132 / 3=44$ if crews and teams would be identical.

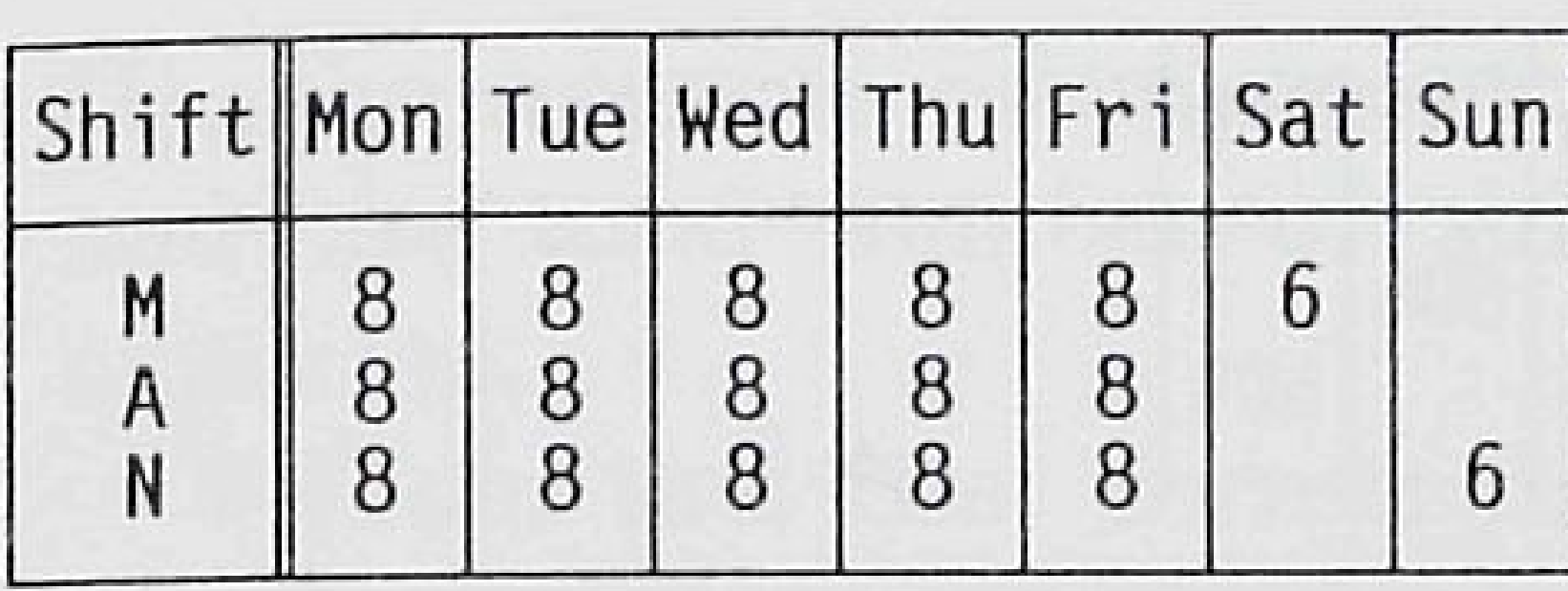

Working hours per shift per day

\begin{tabular}{|c|c||c|c|c|c|c|c|c|}
\hline Function & Shift(s) & Mon & Tue & Wed & Thu & Fri & Sat & Sun \\
\hline 01 & All & 1 & 1 & 1 & 1 & 1 & 1 & 1 \\
02 & All & 5 & 5 & 5 & 5 & 5 & 2 & 2 \\
03 & All & 2 & 2 & 2 & 2 & 2 & 1 & 1 \\
\hline
\end{tabular}

Manning requirements per function per shift per day
Figure 5-5. Working hours and manning requirements

So far we have assigned values to the attributes of the production unit, a crewing system and its shifts. Next, we look at the three teams required by the crewing system. To arrive at an average of 38 working hours per week while maintaining the extended three-crew system, each team should theoretically consist of 1.16 foremen, 5.79 mechanics and 2.23 cleaners. The actual teams therefore consist of 1 full-time foreman, 6 mechanics, one of which can fill in for the foreman when necessary, and three cleaners, one of which works parttime. This can be specified in a table like the one in Figure 5-6. Note that the worker with registration number 440606 appears in two functions.

\begin{tabular}{|c|c|c||c|}
\hline Reg. No. & Team & Function & Contractual hours \\
\hline 390903 & A & 01 & 38.00 \\
440606 & A & 01 & 8.00 \\
440606 & A & 02 & 30.00 \\
530102 & A & 02 & 38.00 \\
630101 & A & 02 & 38.00 \\
670622 & A & 02 & 38.00 \\
690720 & A & 02 & 38.00 \\
741003 & A & 02 & 38.00 \\
140628 & A & 03 & 38.00 \\
760704 & A & 03 & 38.00 \\
891218 & A & 03 & 16.00 \\
\hline
\end{tabular}

Fgure 5-6. Availability of workers for team A

The crewing system includes a number of inconvenient shifts. The weekend shifts are considered to be most inconvenient, which is expressed as $50 \%$ of its working hours, meaning that the inconvenience of working two shifts during the weekend is set equal to that of working three regular shifts of the same length. Similarly, night shifts are rated as 20\% inconvenient (see Figure 5-7). Compensation for inconvenience is one of the labor conditions set by the unions. Thus, the total inconvenience for a three-week shift cycle is 14 hours (five night shifts $\times 8$ hours $\times 20 \%$ plus two weekend shifts $\times 6$ hours $\times 50 \%)$. 


\begin{tabular}{|c|c|c|c|c|c|c|c|}
\hline Shift & Mon & Tue & Wed & Thu & Fri & Sat & Sun \\
\hline M & $20 \%$ & $20 \%$ & $20 \%$ & $20 \%$ & $20 \%$ & $50 \%$ & \\
N & $20 \%$ & $50 \%$ \\
\hline
\end{tabular}

Fgure 5-7. Relative inconvenience per shift per day

For this particular production unit the following labor conditions should be met:

1. Never more than 5 night shifts between two rest periods.

2. Never more than 6 shifts of any kind between two rest periods.

3 . Never less than 2 shifts between two rest periods.

4. No rest periods in the middle of a sequence of night shifts.

5. Near equal number of morning, afternoon and night shifts

6. Equal "distance" between shifts worked on a weekend.

The numbers between parenthesis indicate their relative weight. "Enf" indicates that this condition must be "enforced", meaning that a roster is unacceptable if it at some point violates this condition.

We assume that the rostering period is the entire year of 1989 , which starts on Sunday, January $1^{\text {st }}$, and that crew $C$ will perform the start-up that night. The First day and Last day attributes of the specific instance of ROSTERING PERIOD should then be set to 1-JAN-1989 and 31-DEC-1989 respectively, and offset should be 8 , since the eighth shift of the cycle of crew C in Figure 5-4 corresponds to the Sunday night shift. With the crewing system, these attributes determine the crew roster.

\begin{tabular}{|c|c|c|c|c|c|c|c|c|}
\hline M & A & N & WH & WH+I & AWH & AI\% & WW & WWS \\
\hline 102 & 90 & 102 & 2284 & 2522 & 43.80 & 10.42 & 17 & 34 \\
\hline
\end{tabular}

Figure 5-8. Tallies of the crew roster for crew A

A first correspondence check is to see whether the properties of the crew roster conform to those of the crewing system. Figure 5-8 shows the tallies for the crew roster that results directly from the choice of crewing system and roster period. From these tallies we conclude that the average working hours (AWH) are close enough to the expected 44 . Since there are 52 weeks in 1989 , there will be $52 \div 3=17$ full shift cycles. Crew A started with an afternoon shift on monday, which means that it will also work on the afternoon shift in week 52 . This explains the tallies for the $\mathrm{M}, \mathrm{A}$ and $\mathrm{N}$ shifts $(17 \times 6$ morning and night shifts, $18 \times 5$ afternoon shifts). The total working hours (WH) and inconvenience
(I), the average inconvenience (AI\%), the number of weekends worked (WW) and the total number of shifts worked during a weekend (WWS) all are as expected.

From this first correspondence check we conclude that the make crew roster and compute tallies actions are properly defined in our empirical model. No other actions can be checked at this point, since these actions involve individual rosters, which have not been made yet. In the next section we analyze the task of making individual rosters in depth.

\subsection{TASK ANALYSIS}

Our analysis is based on the data from the empirical model we described in the previous section. Thus, the personnel manager must make an individual roster for each of the 30 workers. His planning method is heuristic in nature, and his tools are a pencil, an eraser and a large sheet of 5 millimeter grid paper.

The first thing the personnel manager does is to process the notifications of absence by marking each given worker/day/shift combination with an appropriate code. For simplicity's sake we assume that no absence is known beforehand.

Since the crewing system assigns the three teams always to different shifts, each of the three 10-worker teams can be planned separately. For each team the personnel manager first makes rosters for the foreman and the skilled mechanic, then for the other five mechanics, and finally for the cleaners. When making rosters for these "function groups", he starts with the inconvenient shifts, because these shifts must be distributed evenly, both in time and over the workers, to obtain fair rosters.

For the function of foreman 46 working hours per week are available (see Figure 5-6), but only 44 are needed. Consequently, the foreman will work for about 36.2 hours, and the mechanic for 7.8. A quick calculation shows that the foreman should work 28 of the 34 weekend shifts, while the mechanic should work 6 shifts as a foreman, and his remaining 22 weekend shifts as a mechanic. Similarly, the foreman will have to work 70 of the 85 night shifts (102 minus 17 Sundays), meaning that the mechanic will have to replace him in 15 shifts. The personnel manager distributes these 21 inconvenient shifts evenly over the year to make the foreman's roster as balanced as possible.

These shifts now appear on the stand-in foreman's roster. This roster must be "filled out" with shifts as a mechanic. The need for mechanics equals 208 working hours, while 220 are available. The stand-in foreman should therefore work an average of $30 / 220 \times 208 \approx 28.4$ hours per week, the full-time mechanics 35.9 , which translates as approximately 8 and 12 weekend shifts, and 61 and 79 night shifts, respectively. The personnel manager fills out the stand- 
in foreman's roster with his 69 inconvenient shifts, and then assigns 91 inconvenient shifts to each of the five full-time mechanics.

For the cleaner function 92 working hours per week are available, but only 84 are needed. The part-time cleaner should therefore work $16 / 92 \times 84 \approx 14.6$ hours, the two full-time cleaners 35.7. This translates as 6 and 14 weekend shifts, and 18 and 42 night shifts, respectively. Once these have been assigned, all inconvenient shifts have been rostered. The morning and afternoon shifts, in so far as they do not fall in a weekend, are scheduled in a similar fashion. Figure 5-9 shows part of the individual rosters for crew A.

\begin{tabular}{|c|c|c|c|c|c|c|c|c|c|c|c|c|c|c|}
\hline Fore & & Week 1 & Week 2 & & & & & & & & & & & \\
\hline Reg. No. & Hrs. & MTWTFSS & MTWTFSS & MTWTFSS & MTWTFSS & M & A & N & WH & $\mathrm{WH}+\mathrm{I}$ & AWH & $A I \%$ & $W W$ & WWS \\
\hline $\begin{array}{l}390901 \\
440606\end{array}$ & $\begin{array}{r}38.0 \\
8.0\end{array}$ & $\mid \begin{array}{l}\text { AAAAA-- } \\
--22-\end{array}$ & $\mid \begin{array}{l}\text { MMMM--N } \\
----M M 2\end{array}$ & & & $\begin{array}{l}85 \\
17\end{array}$ & $\begin{array}{l}75 \\
15\end{array}$ & $\begin{array}{l}85 \\
17\end{array}$ & $\begin{array}{r}1902 \\
382\end{array}$ & $\begin{array}{r}2101 \\
421\end{array}$ & $\mid \begin{array}{r}36.47 \\
7.32\end{array}$ & $\left|\begin{array}{l}10.46 \\
10.20\end{array}\right|$ & $\begin{array}{r}16 \\
4\end{array}$ & 5 \\
\hline
\end{tabular}

\begin{tabular}{|c|c|c|c|c|c|c|c|c|c|c|c|c|c|c|}
\hline Mer & & eek 1 & & & ek & & & & & & & & & \\
\hline & & MTWTFSS & MTWTFSS & MTWTFSS & MTWTFSS & M & A & $\mathrm{N}$ & WH & $W H+I$ & AWH & $A I \%$ & $W W \mid$ & WWS \\
\hline $\begin{array}{l}440606 \\
530102 \\
630101 \\
670622 \\
690720 \\
741003\end{array}$ & $\begin{array}{l}38 . \\
38 . \\
38 . \\
38\end{array}$ & & $\begin{array}{l}----11 N \\
\text { MMMMM-- } \\
\text { MMMMM-- } \\
\text { MMMMM-N } \\
\text { MMMMMM- } \\
\text { MMMMMM- }\end{array}$ & & & $\begin{array}{l}63 \\
80 \\
79 \\
80 \\
79 \\
80\end{array}$ & $\begin{array}{l}63 \\
79 \\
80 \\
80 \\
79 \\
78\end{array}$ & $\begin{array}{l}63 \\
78 \\
80 \\
79 \\
80 \\
80\end{array}$ & & & & $\begin{array}{l}8.16 \\
8.26 \\
8.00 \\
8.21 \\
8.21\end{array}$ & $\begin{array}{r}10 \\
10 \\
8 \\
9 \\
10\end{array}$ & $\begin{array}{l}7 \\
13 \\
12 \\
12 \\
12 \\
12\end{array}$ \\
\hline
\end{tabular}

\begin{tabular}{|c|c|c|c|c|c|c|c|c|c|c|c|c|c|c|}
\hline \multicolumn{2}{|c|}{ 3. Cleaner } & Week 1 & Week 2 & Week 3 & Week 4 & \multicolumn{9}{|c|}{ Tallies } \\
\hline Reg. No. & Hrs. & MTWTFSS & MTWTFSS & MTWTFSS & MTWTFSS & M & A & $\mathrm{N}$ & WH & $W H+I$ & AWH & $A I \%$ & $\mid W W$ & WWS \\
\hline $\begin{array}{l}140628 \\
760704 \\
891218\end{array}$ & $\begin{array}{l}38.0 \\
38.0 \\
16.0\end{array}$ & $\left\{\begin{array}{l}\text { AAA-- } \\
\text { AAAAA- } \\
---A--\end{array}\right.$ & $\mid$\begin{tabular}{l} 
MMMM--N \\
MMMMM-- \\
\hdashline$---M M-$
\end{tabular} & $\mid \begin{array}{l}\text { NNNNN-- } \\
-N N N N-- \\
\text { N------ }\end{array}$ & $\begin{array}{l}-A A A A-- \\
\text { AAA-- } \\
---A A--\end{array}$ & $\begin{array}{l}65 \\
64 \\
64\end{array}$ & $\begin{array}{l}61 \\
62 \\
62\end{array}$ & $\begin{array}{l}62 \\
63 \\
63\end{array}$ & \begin{tabular}{|l|}
1482 \\
1490 \\
1488
\end{tabular} \mid & $\begin{array}{l}1606 \\
1614 \\
1615\end{array}$ & $\left|\begin{array}{l}28.42 \\
28.57 \\
28.53\end{array}\right|$ & $\begin{array}{l}8.38 \\
8.33 \\
8.54\end{array}$ & $\left|\begin{array}{l}7 \\
8 \\
8\end{array}\right|$ & $\begin{array}{l}11 \\
11 \\
12\end{array}$ \\
\hline
\end{tabular}

Figure 5-9. Individual rosters for crew A from January $2^{\text {nd }}$ through $29^{\text {th }}, 1989$

The overall flow of personnel planning for a production unit is depicted in Figure 5-10. First, the personnel manager makes the crew roster, which will serve as the reference roster. Secondly, he marks the absence of workers by marking their individual rosters with appropriate codes on the days specified in the notifications of absence. He then groups the workers according to the function(s) they are to perform. Changes in the rosters of one function group will never affect the rosters in some other function group, so no coordination is needed, other than to make sure that no function groups are omitted.

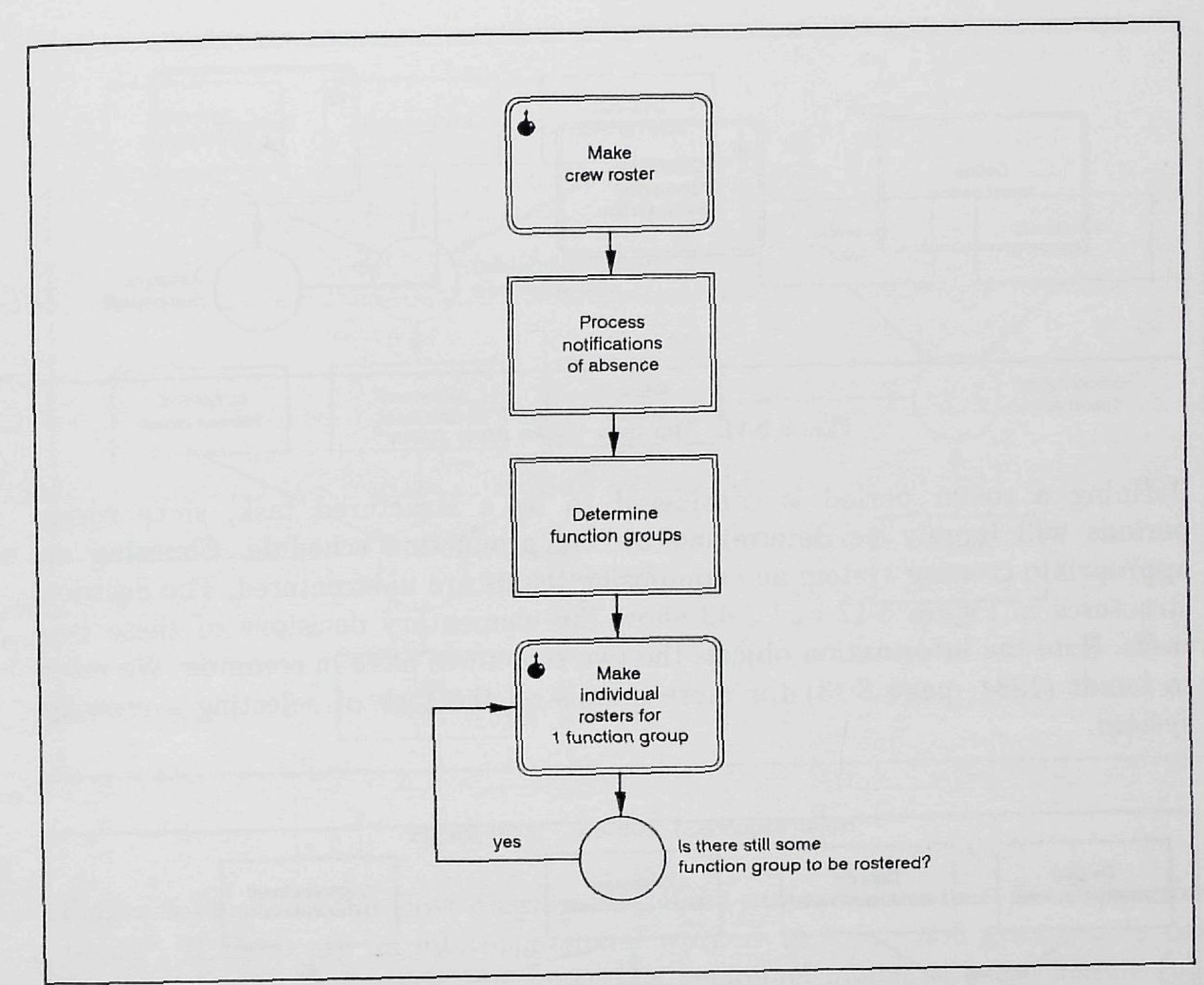

Fgure 5-10. The task "Plan personnel"

"Process notifications of absence" and "Determine function groups" are structured tasks that require no further analysis. The other two subtasks are more interesting.

Figure 5-11 shows the flow diagram of "Make crew roster". Making a crew roster involves three subtasks: A roster period has to be chosen, an appropriate crewing system has to be determined, and teams must be composed. It is difficult to define a general coordination mechanism for these tasks. The instruments and it is impossible to specify under which circumstances the roster periods will determine the crewing systems, and vice versa. 


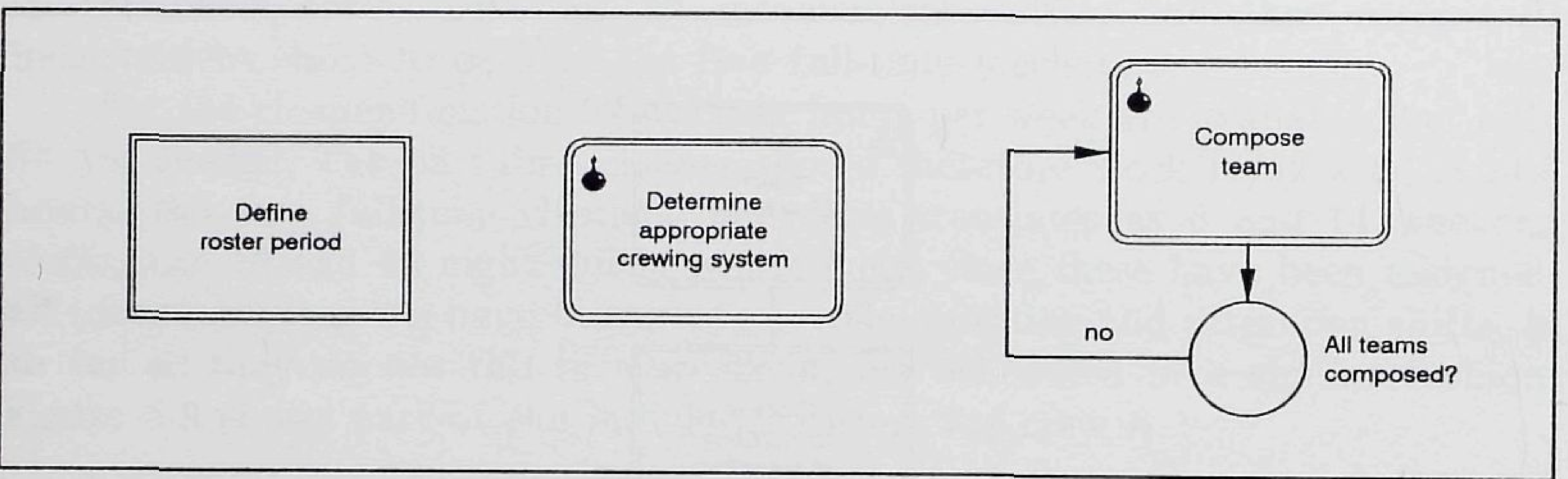

Flgure 5-11. The task "Make crew roster"

Defining a roster period is considered to be a structured task, since roster periods will largely be determined by the production schedule. Choosing an appropriate crewing system and composing teams are ill-structured. The decision structures in Figure 5-12 and 5-13 show the elementary decisions of these two tasks. Note the information objects the two structures have in common. We refer to Lundt (1984, page 8-13) for more details on the task of selecting a crewing system.

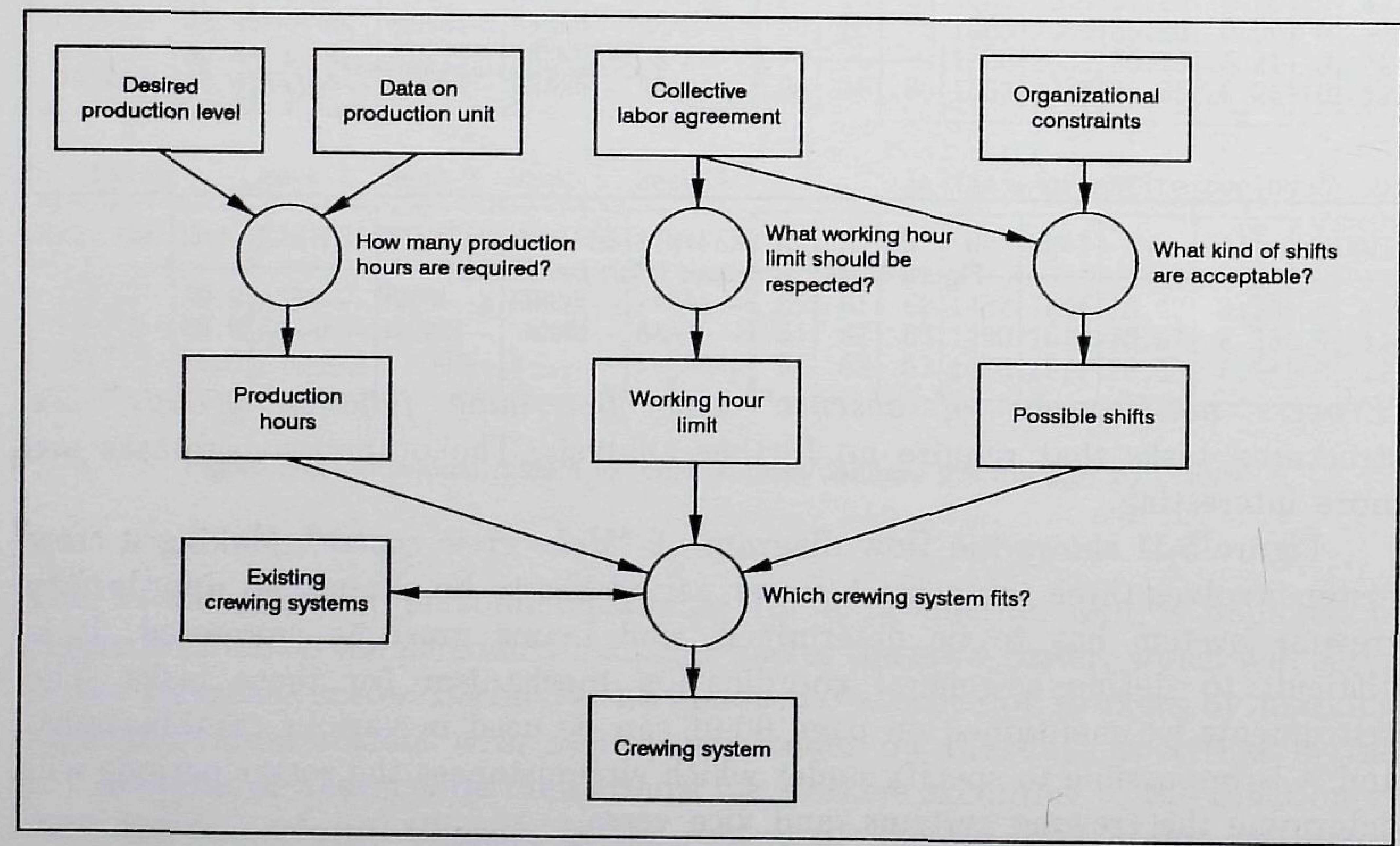

Fgure 5-12 The task "Determine appropriate crewing system"

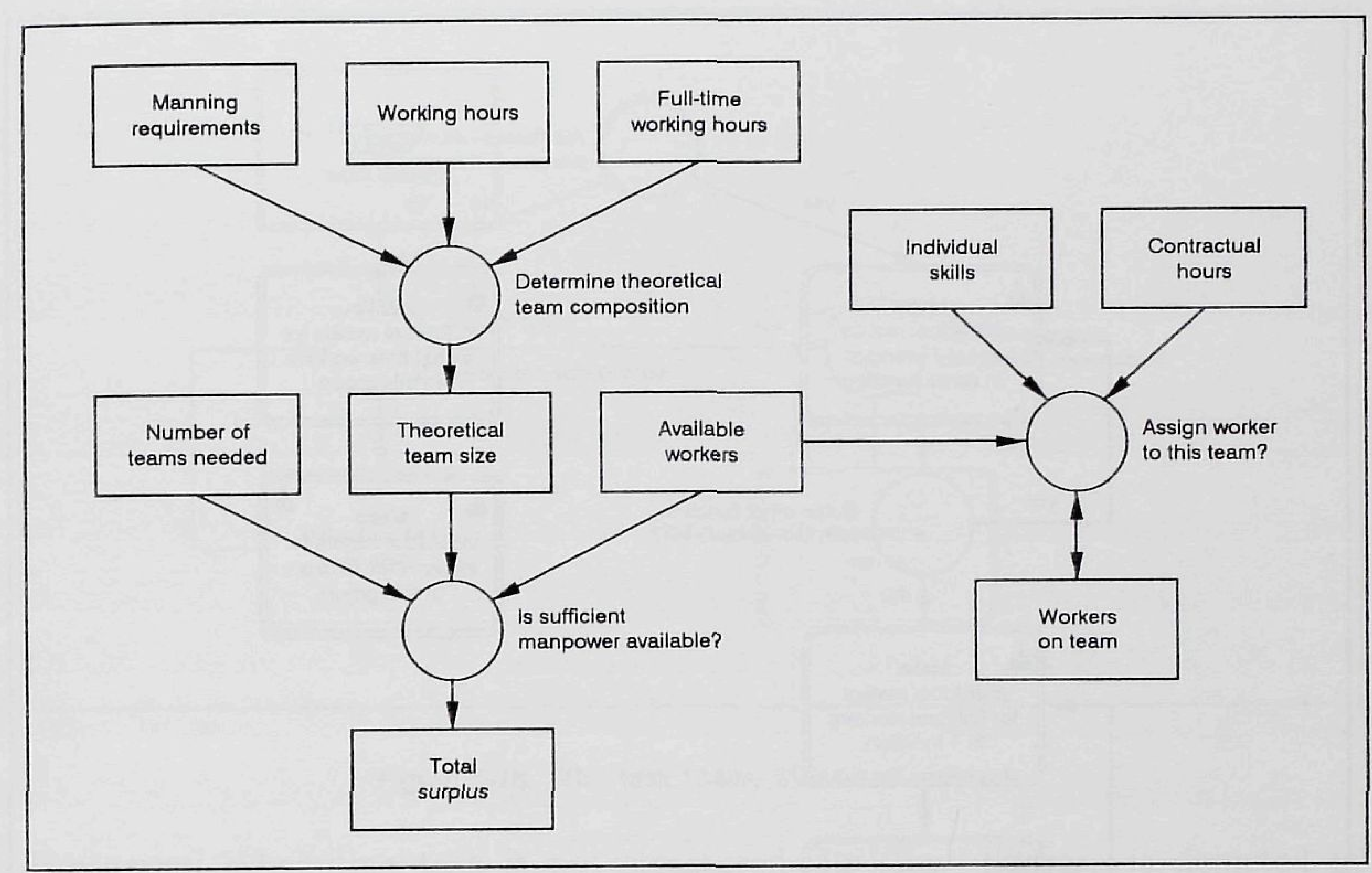

Flgure 5-13. The task "Compose team"

Figure 5-14 shows the flow diagram of "Make individual rosters for 1 function group". If there are no multi-functional workers in a function group, only one function has to be rostered. In general, a personnel manager will schedule fulltime workers first because part-time workers are entitled to more days off, which makes their rosters more flexible.

Things are more complicated when a function group involves more than one function. In such situation, the multi-functional workers are rostered first. This may seem illogical, since workers with many skills would seem to be quite convenient for "filling holes" in a near-finished roster, and therefore should be rostered last. The problem, however, is that the personnel manager is bound by a multi-functional worker's contractual hours. This attribute determines exactly how many shifts a worker should be working in each of his functions. As a result, the rosters of multi-functional workers are most difficult to balance.

Once all multi-functional workers have been scheduled, the rosters for each of the functions are completed in much the same way rosters are made for function groups without multi-functional workers: First the full-time workers are scheduled, then the part-time workers. 


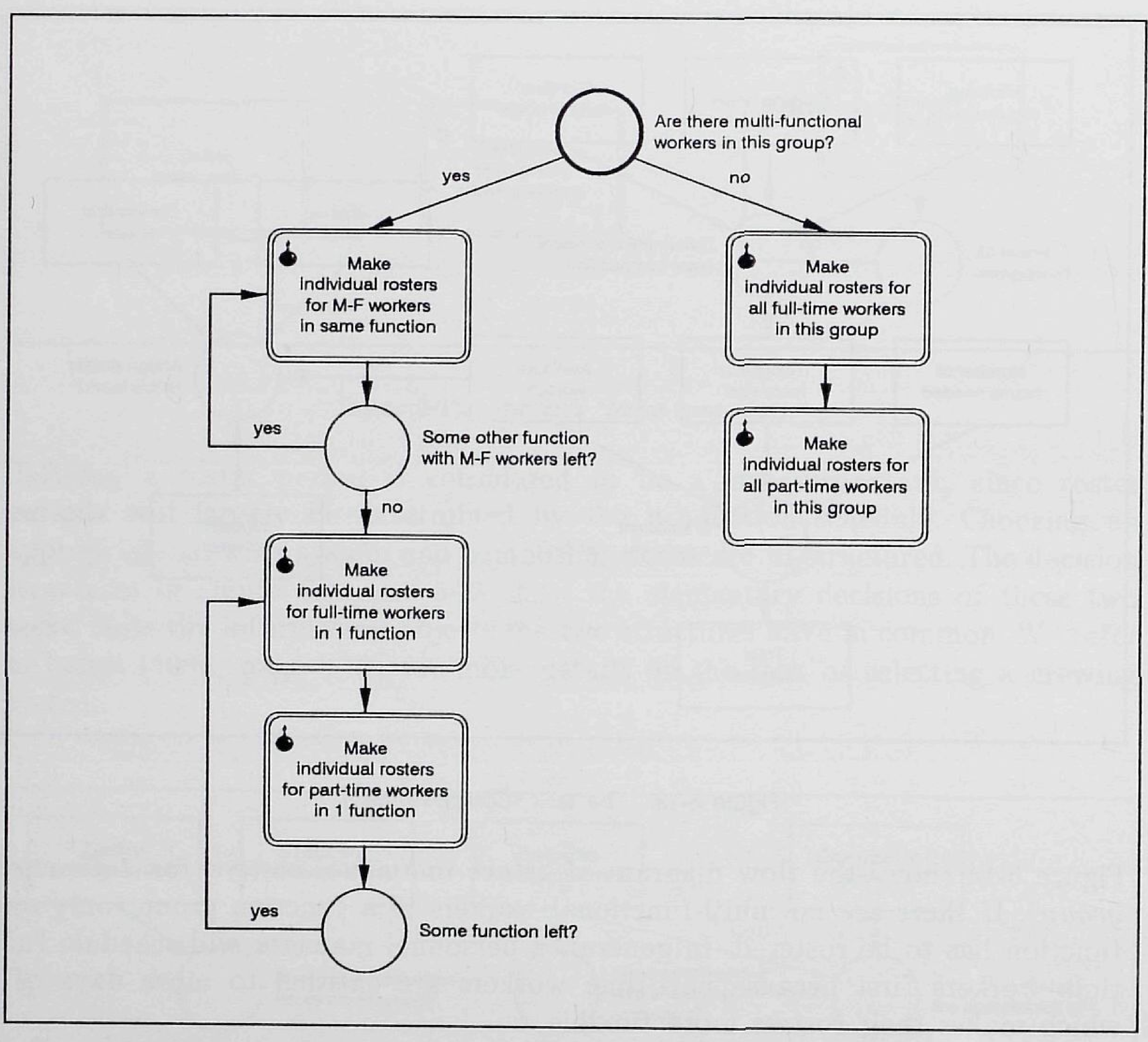

Fgure 5-14. The task "Make individual rosters for 1 function group"

Observe that in our example, the foreman/mechanic is the reason why there are two function groups: the foremen and mechanics in one function group, and the cleaners in the other. If there also had been a mechanic/cleaner, there would have been but one function group.

Although the type of worker is different for each subtask in Figure 5-14, these tasks all come down to making individual rosters. For this reason, each subtask has the same alias: "Make individual rosters". The flow diagram of this task is shown in Figure 5-15. The first step is to determine the relative work loads. Being a straightforward computation, similar to those on page 103, this is a structured task.

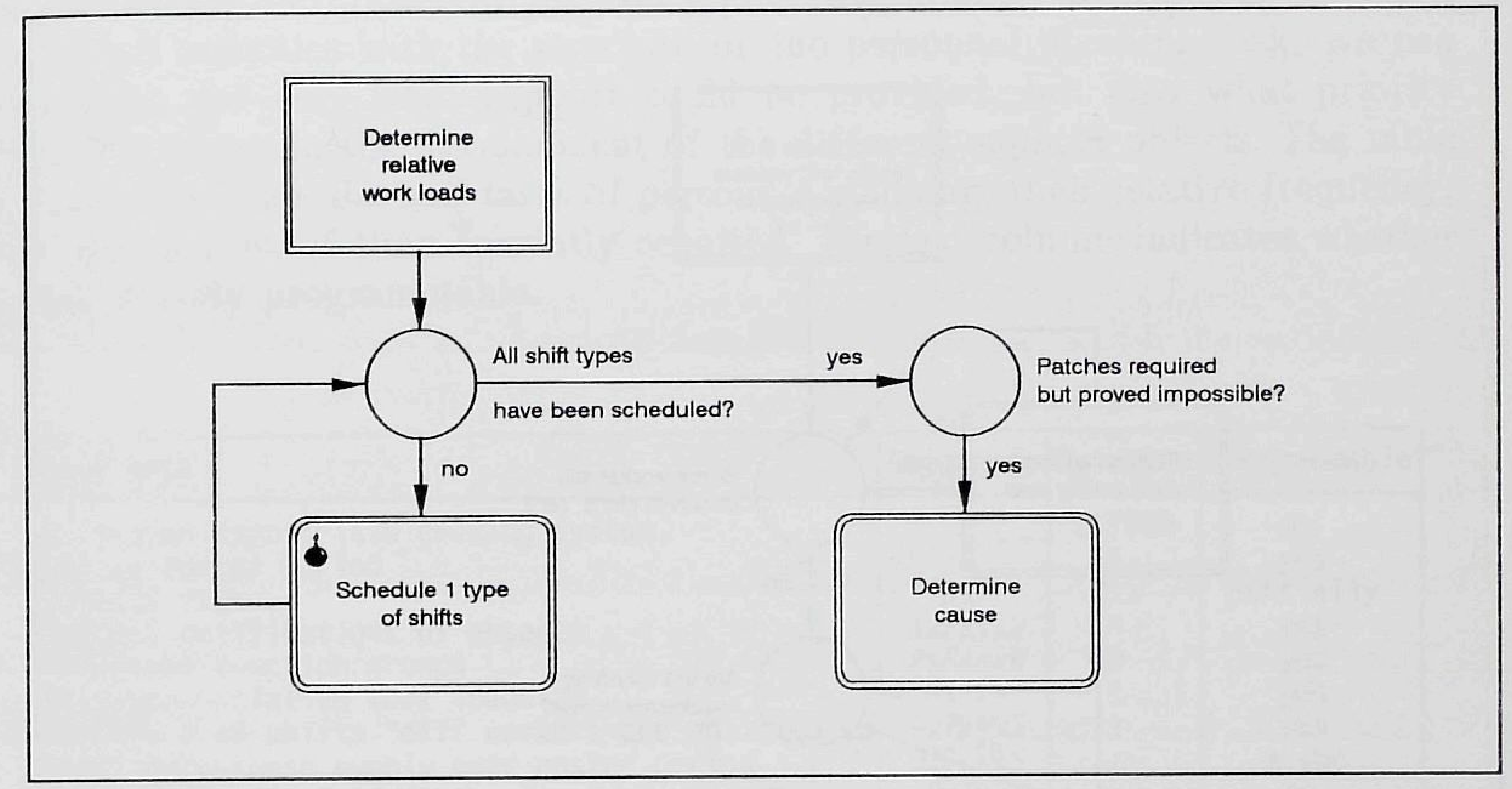

Flgure 5-15. The task "Make individual rosters"

The actual scheduling task is not structured. Its flow diagram is depicted in Figure 5-16. The first subtask of "Schedule 1 type of shifts" is again purely computational, and the checks represented by three coordinating decisions might also be performed algorithmically. But the uniform distribution of shifts is a process of trial-and-error in which the personnel manager tries to "patch" rosters on those places where labor conditions are violated, where rosters become too unfair, or where the manning requirements are not met.

We shall now use the task structure represented by Figures 5-10 through 5-16 to pinpoint bottlenecks in the rostering process. Most of the personnel managers we observed are very experienced planners, who know the characteristics of specific production units and crewing systems by heart. Major changes in production hours, and therefore crewing systems, are usually not considered. Almost without exception, reduction of average working hours is achieved by increasing team sizes. The tasks "Determine appropriate crewing system", "Define roster period", and "Compose teams" therefore take relatively little time.

When making individual rosters, personnel managers tend to use the following heuristic approach: Having calculated the exact number of shifts "off" each individual is entitled to, they spread these shifts evenly over the roster 


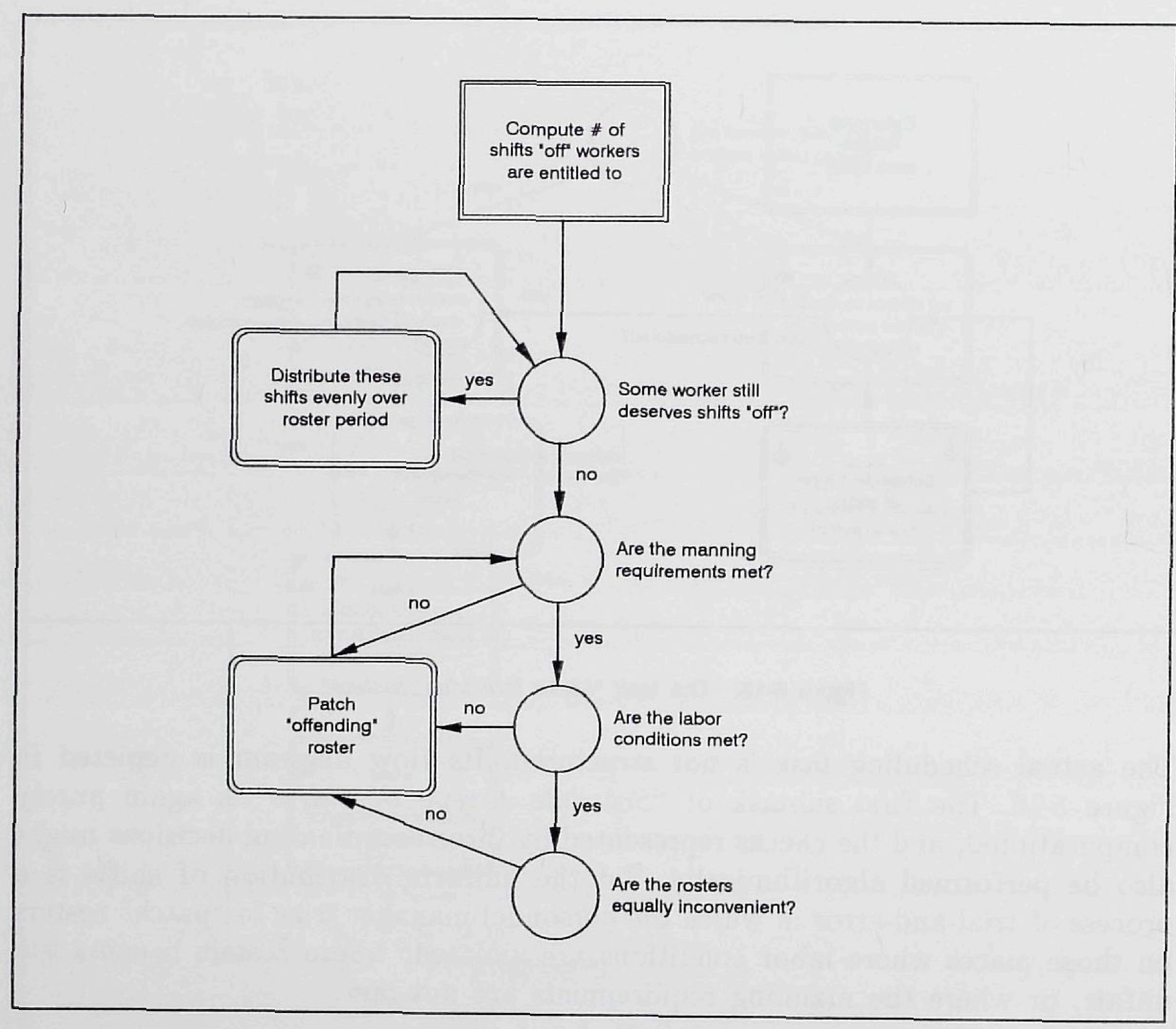

Fgure 5-16. The task "Schedule 1 type of shifts"

period using rules like "De Wit and Van de Velde still have the highest tally? Then one of these two gets this shift off" and "De Wit did 4 night shifts three weeks ago, and Van de Velde did 5, so it must be Van de Velde". Labor conditions are not checked explicitly. Tallies are kept by ticking off the shifts each worker was entitled to.

Once all shifts "off" have been allotted, resulting in what one might call a "tentative" team roster, the exact tallies (including things like weekend shifts and inconvenience) are calculated, and labor conditions are checked. Then the process of moving shifts around starts. This "patching" of the team roster, which continues until all individual rosters are acceptable, involves a tedious amount of calculation - tedious because it has to be done by hand.
By combining our observations concerning the amount of time spent on a particular activities with the structure of the personnel planning task, we can determine not only what support could be provided, but also what priority should be given to the development of the different support objects. The table in Figure 5-17 lists the leaf tasks of personnel planning, their relative frequency, and the amount of time currently required. The last column indicates whether a task is fully programmable.

\begin{tabular}{|l|c|c|c|}
\hline Leaf task & Frequency & Duration & Programmable \\
\hline Determine appropriate crewing system & $P$ & $1-1000$ & no \\
Define roster period & $P$ & 1 & yes \\
Compose team & $P \times T$ & $3 \times W$ & partially \\
Process notifications of absence & axPXTXW & 5 & yes \\
Determine function groups & $P \times F \times m \times W$ & 10 & yes \\
Determine relative work loads & $P \times T \times W$ & 1 & yes \\
Compute \# of shifts "off" workers are entitled to & $P \times T \times W \times S$ & 1 & yes \\
Distribute these evenly over roster period & $P \times T \times H \times S$ & $n$ & maybe \\
Patch "offending" roster & $P \times T \times W$ & $C \times W$ & no \\
\hline
\end{tabular}

$\rho_{:} \neq$of product ion units, $T: \#$ of teams, w: \# of workers on a team, $F:$ \# of functions, $S: \#$ of shifts
a: average absence per worker, m: multi-functionality per worker, $n:$ number of shifts "off", $c:$ complexity

Flgure 5-17. Leaf tasks of "Plan personnel"

The duration is expressed in units of 5 seconds. The wide range for "Determine appropriate crewing system" represents the fact that this task is very simple when a roster has to be made for a production unit that has been manned according to the same crewing system for years, but much more time-consuming when major changes in the attributes of a production unit requires complete reinvestigation of the advantages and disadvantages of alternative crewing systems.

Using the frequencies and durations from Figure 5-17 we can calculate the expected duration of "Plan personnel" in the empirical model described in the previous section. This model involved one production unit, three shifts, three functions, and three teams of 10 workers each. If we combine the shift cycle in Figure 5-4 with the manning requirements in Figure 5-5 we find that a team roster for one year with no surplus manpower contains $17 \times 2 \times(1+2+1)=136$ shifts during the weekend, and $52 \times 5 \times(1+5+2)=2080$ other shifts, which amounts up to 221.6 shifts per worker. From Figure 5-8 we learn that the crew roster contains 294 shifts. Thus, the number of shifts "off" to be distributed equals 72.4 per worker. Since there is one multi-functional worker in each team the degree of multi-functionality $m$ equals 0.1 . We disregard possible absence of workers, so $a=0$. If we substitute these numbers for the variables in the 
expressions for frequency and duration in Figure 5-17, we can calculate the total duration $D$ of "Plan personnel" as follows:

$$
\begin{aligned}
D & =P \times(1+1+W \times(F \times m \times 10+T \times(3+a \times 5+1+S \times(1+n)+c \times W)) \\
& =1 \times(1+1+10 \times(3 \times 0.1 \times 10+3 \times(3+0 \times 5+1+3 \times(1+72.4)+c \times 10) \\
& =6758+300 c
\end{aligned}
$$

Even if we assume that $c=10$, which would mean that the personnel manager can locate and patch an "offending" spot in an individual roster in less than a minute, $D$ would equal 9758 time units, or well over 13 hours.

How would computerized support affect $D$ in this example? The table in Figure 5-18 shows for each programmable leaf task what time savings would be made if that task could be performed by a computer in less than one time unit. Clearly, even fully automating the programmable tasks would result in a small half hour time savings - less than $2 \%$ of the total duration. Supporting the choice of an appropriate crewing system might boost this percentage, but never over $10 \%$. The bottleneck is the heuristic rostering process.

\begin{tabular}{|l|c|}
\hline Leaf task & Potential time savings \\
\hline Determine appropriate crewing system & between 0 and $1 \frac{1}{2}$ hours \\
Define roster period & none \\
Compose team & 7.5 minutes \\
Process notifications of absence & 6.3 minutes \\
Determine function groups & 2.5 minutes \\
Determine relative work loads & 2.5 minutes \\
Compute \# of shifts "off" workers are entitled to & 7.5 minutes \\
Distribute these evenly over roster period & 9 hours \\
Patch "offending" roster & 4 hours or more \\
\hline
\end{tabular}

Fgure 5-18. The impact of computerized support on the duration of "Plan personne/"

\subsection{DECISION SUPPORT}

As we indicated in Figure 5-17, we might be able to support the personne manager in the last two tasks. If we provide him with a spreadsheet-like too that would replace his pencil and paper, and compute various tallies, this would likely result in considerable time savings. If we are able to capture some of the personnel manager's expertise in a rule-based system, time savings would increase dramatically. With this in mind we decided to develop the so-called "roster worksheet" and "automatic pilot", which we will describe in this section.
The roster worksheet

The functional specifications of this support component are in the first place based on the actions specified in the definition of PERSONNEL MANAGER on page 97. Many actions of the personnel manager involve instantiating object classes like TEAM WORKER, SHIFT, etcetera, and defining the attribute values of the created instances. To facilitate these actions, the support class TABLE is defined. The primary functions of a table are to display and allow fast and easy modification of the attribute values of the instances of a particular information class, and to create new instances or delete existing ones.

\section{object class
[ attributes \\ Type}

Fields

Key fields

Position

Size

Number of lines

Contents

First visible object

Selected object Selected field

\section{actions}

change selected object/field

add new object to Contents

delete object from Contents

scroll up/down disappear from screen

accept user input
Indicates whether this table is fixed size, or whether object may be added

A list of field objects that defines which objects/ attributes can be accessed through this table.

The fields that together constitute a key in the combination of their values must be unique. The place on the screen where this table has to appear.

The rectangle this table will cover on the screen The maximal number of objects that will simultaneously be visible in this table.

A list of information objects that can be accessed through this table.

The object currently displayed on the first line of this table.

The object currently selected by the user. The field currently selected by the user.

The current selection is highlighted.

By changing the value of First visible object. User input strings are sent to the currently selected field object, and processed there. sense that for each object in this table the

Instances of class TABLE provide an interface between the decision maker and a collection of information objects of the same class. A table is divided into a number of columns, each of which corresponds to a specific attribute of that class. Tables appear on the screen in a format similar to that of the tables in Figure 5-5 through 5-7. Each line in a table corresponds to one object. 
The specific input/output properties of the fields in one column are defined by instances of the support class FIELD.

$\begin{aligned} & \text { object class FIELD } \\ & \text { [ attributes }\end{aligned}$
$\begin{array}{ll}\text { Header } & \text { The text to be displayed at the top of this field column } \\ \text { Reference } & \text { this field } \\ \text { Size } & \text { The rectangle this field will cover on the screen } \\ \text { Margin } & \text { To be able to center values within that rectangle } \\ \text { Access } & \text { Indicates whether the contents of this field may be changed } \\ \text { Test } & \text { Defines which input is allowed in (for example, numeric input } \\ & \text { only) }\end{array}$

\section{actions}

accept input string

If the specified test is passed, the value of the attribute referred to by Reference is changed.

With the help of the appropriate tables the personnel manager can enter all information related to a particular rostering problem, with the exception of crewing systems, team rosters and individual rosters. The purpose of the support class WORKSHEET is to facilitate the creation and manipulation of these rosters. A worksheet may contain different types of objects. The worksheet in Figure 5-19, for example, holds integers of various sizes and fixed point decimals, and character strings, each of which requires specific input tests. Moreover, only certain cells may be modified by the personnel manager (in this case the shif codes; the values of the other cells are either calculated or input via tables).

\begin{tabular}{|c|c|c|c|c|c|c|c|c|c|c|c|c|c|c|c|c|c|}
\hline N & WH & AI\% & Reg. No. & Hrs. & Mo $\mathrm{Tu}$ & $u$ We & Th & $\mathrm{Fr}$ & Sa & Su Mo & o $\mathrm{Tu}$ & We & Th F & $\mathrm{Fr} \mathrm{Sa}$ & & Mo & Tu \\
\hline $\begin{array}{l}62 \\
79 \\
80 \\
78 \\
80 \\
80\end{array}$ & $\begin{array}{l}1468 \\
1868 \\
1854 \\
1866 \\
1880 \\
1872\end{array}$ & $\begin{array}{l}8.47 \\
7.94 \\
8.40 \\
7.85 \\
8.12 \\
8.33\end{array}$ & $\begin{array}{l}440606 \\
530102 \\
630101 \\
670622 \\
690720 \\
741003\end{array}$ & \begin{tabular}{|}
30.0 \\
38.0 \\
38.0 \\
38.0 \\
38.0 \\
38.0
\end{tabular} & $\begin{array}{ll}\bar{A} & \bar{A} \\
A & A \\
A & A \\
A & A \\
A & A\end{array}$ & $\begin{array}{l}- \\
\bar{A} \\
A \\
A \\
A\end{array}$ & $\begin{array}{l}A \\
\bar{A} \\
A \\
A \\
A\end{array}$ & $\begin{array}{l}A \\
\bar{A} \\
A \\
A \\
A\end{array}$ & $\begin{array}{l}- \\
\overline{-} \\
\overline{-} \\
\overline{-}\end{array}$ & $\begin{array}{ll}- & \bar{M} \\
= & M \\
- & M \\
- & M \\
- & M\end{array}$ & $\begin{array}{l}\bar{M} \\
M \\
M \\
M \\
M\end{array}$ & $\begin{array}{l}\bar{M} \\
M \\
M \\
M \\
M\end{array}$ & $\begin{array}{ll}M & M \\
M & - \\
M & M \\
M & M \\
M & M \\
M & M\end{array}$ & $\begin{array}{ll}M & M \\
\bar{M} & - \\
M & - \\
M & \bar{M} \\
M & \bar{M}\end{array}$ & $\begin{array}{l}N \\
N \\
- \\
- \\
- \\
-\end{array}$ & $\begin{array}{l:l}N & \\
N & \\
N & \\
N & \\
N & \end{array}$ & $\begin{array}{l}\bar{N} \\
N \\
N \\
N \\
N\end{array}$ \\
\hline & & & & & $\mid \begin{array}{ll}5 & 5 \\
0 & 0\end{array}$ & & & $\begin{array}{l}5 \\
0\end{array}$ & & 0 & $\begin{array}{l}5 \\
0\end{array}$ & $\begin{array}{l}5 \\
0\end{array}$ & $\begin{array}{ll}5 & 5 \\
1 & 0\end{array}$ & $\begin{array}{ll}5 & 2 \\
0 & 0\end{array}$ & $\begin{array}{l}2 \\
0\end{array}$ & $\begin{array}{l}5 \\
0\end{array}$ & $\begin{array}{l}5 \\
0\end{array}$ \\
\hline
\end{tabular}

Fgure 5-19. A worksheet for making individual rosters

\section{object class WORKSHEET \\ [ attributes}

$\begin{array}{ll}\text { Name } & \text { The name of this worksheet (it will be displayed on the screen) } \\ \text { Position } & \text { The screen coordinates of this worksheet's upper left corner. }\end{array}$

Position

Size

Active matrix The cell matrix currently holding the cursor.

The rectangle this worksheet will cover on the screen.

\section{actions}

accept user input

send user input to the active cell matrix

\section{object class CELL MATRIX}

[ attributes

Worksheet

Rows

Nimber of visible

Number of columns

Refers to the worksheet this matrix is part of.

A list of objects (the actual cells of this matrix).

A matrix may contain more rows than appear on the screen.

The row that appears at the top of this matrix' rectangle.

The number of cells in each row (must be the same for each

row).

First visible column

The rows of a

Mask

of this matrix' rectangle.

of the cell in the upper left corner

that are to be displayed. In our rostering example, this masking facility is useful if the personnel planner wishes to see for example only the weekends, or those days of a team roster on which the crew will be working a night shift.

The cell(s) selected by the user.

Selection

Position

Size

The position of the upper left corner of this matrix, relative to the position of the worksheet it is part of.

Access

The rectangle this matrix covers on the screen.

Determines whether the contents of matrix cells may be changed. If such access is denied, this matrix can never become active

Test

Defines how input strings for this matrix should be tested.

\section{actions}

display rows

scroll up/down

scroll left/right

Shows the contents of each of its cells (insofar as they are visible) in their appropriate position on the screen.

All matrices that are "connected" with the same vertical scroll bar will also be induced to scroll.

All matrices that are "connected" with the same horizontal

scroll bar will also be induced to scroll.

\section{se lect block}

change number of visible rows

change number of visible columns 
A worksheet therefore is defined in terms of so-called "cell matrices". Each cell matrix consists of objects of the same class, and has its own test for checking input strings. These objects (the actual cells) must all be capable of performing the action return contents in string format. In that way a single display action can be defined for the support class CELL MATRIX. The worksheet accepts all user input, and determines which cell matrix currently holds the cursor, and sends user input strings to that matrix.

The "roster worksheet" support component has been implemented using eighteen instances of TABLE and two instances of WORKSHEET. The results of our analysis of the personnel planning task have had considerable impact on the way these instances are initialized, and the sequence in which they are activated. The layout of these tables and the menu structure have been designed to reflect as much of the task structure of "Plan personnel" as is possible. We describe neither the specific tables nor the menu structure here, but refer to the ROPAQ ${ }^{\mathrm{TM}}$ demonstration software in directory $\backslash \mathrm{RP}$ on the diskette that came with this thesis instead.

\section{The automatic pilot}

In view of the potential time savings listed in Figure 5-18, an efficient algorithm that distributes shifts "off" evenly over the individual workers and the roster period would in theory reduce the time required for the personnel planning task by some 9 hours. If this algorithm would also take into account some or all of the labor conditions, the time required for patching rosters would drop as well. Our aim, therefore, is to find such an algorithm.

We decide against using optimization techniques, for two reasons. First quick response is considered to be more important than optimal solutions, and algorithmic optimization is time-consuming. Secondly, experiences with optimizing algorithms are that the solutions found are difficult to understand for a human decision maker, and therefore difficult to modify by hand. We fully agree with Little (1970, page 483) in that models should be designed in such a way that their users feel that they "own" the model in the sense that they can relate its output to the input they provide it with. Our assumption is that when a small set of heuristic rules is used, the output is easier to understand.

We use our task analysis technique to design an alternative task structure for the task "Make individual rosters". This structure is based on the following three observations:

1. According to the task diagrams in Figure $5-15$ and $5-16$, the approach to making individual rosters consists of calculating the number of shifts "off" by expressing both manning requirements and available manpower in shifts and subtracting the former from the latter, and then distributing these shifts "off" evenly over both workers and roster period.

Since manning requirements have to be met at all times, one could obtain identical results by looking at one day of the roster period at the time, calculating the surplus manpower for that day, and then deciding which workers are best given a shift "off" to reduce the surplus to 0.

2. A personnel manager schedules one type of shifts at a time. Suppose he starts with the night shifts. Since the aim is a balanced distribution of shifts over the workers, each worker will be scheduled for the same number of night shifts (give or take one shift). This means that when the personnel manager starts scheduling the afternoon shifts, he needs not consider the rosters for the night shifts. In other words, the rosters for one shift type have little or no influence on the rosters for another type of shift.

Consequently, one could obtain identical results by looking at one day of the roster period at a time - even if this means alternating between different shift types - provided that the decision which workers will get a $\mathrm{N}$-, A- or M-shift "off" is based on the corresponding tally, and not on the total number of shifts the workers have been scheduled for

3. Shifts "off" that directly follow or precede a rest period are valued more highly than those that fall in the middle of a "block" of shifts. Thus, the roster "-AAAAA---MMMMM-" is preferred over "-AAAAA--MM-MM-". Moreover, there may be labor conditions that explicitly forbid "split blocks", especially when the night shift is concerned. For this reason, a personnel manager tends to first look at the days at the beginning and end of a block, and proceed from both ends towards the middle of the block.

The same results are obtained if one starts at the beginning of a block, moving from the left towards the center, then resumes at the end of the block, moving from the right towards the center.

Before we can design a new task structure, we have to extend our conceptual model of the personnel planning problem with the "artificial" object class BLOCK:

\section{object class BLOCK \\ [ attributes}

First day

Last day

Center

First direction
The first day of this block, relative to the first day of the crewing system. The last day of this block, relative to the first day of the crewing system.

es at which point the personnel manager switches directions.

Indicates whether the personnel manager starts with the first day or the last day of the block. 
A block specifies a number of consecutive days within the shift cycle of a particular crewing system. Figure 5-20 shows how the shift cycle of the extended three-crew system from Figure 5-4 is divided into three blocks. The vertical bars "l" separate the last day of one block from the first day of the next block. The arrows mark the center of a block and indicate the initial direction (" " means "First right to left until the center is reached, then left to right", and "»" means the other way around). Note that the initial direction of the block of night shifts is opposite to that of the block of morning shifts. This represents the fact that in this particular situation the personnel manager prefers to deal with shifts on Saturdays and Sundays first.

\begin{tabular}{|c|c|}
\hline Crew & Shift cycle \\
\hline $\begin{array}{l}A \\
B \\
C\end{array}$ & 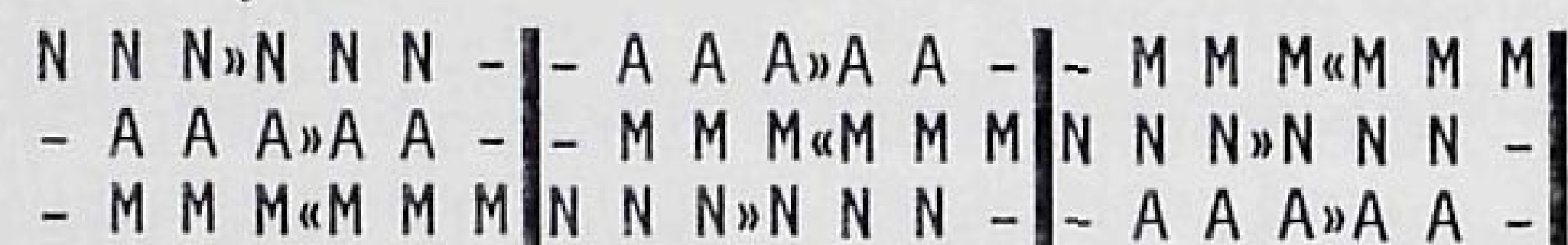 \\
\hline
\end{tabular}

Figure 5-20. Block boundaries in the extended three-crew system

When the crew roster is constructed, based on the crewing system, the selected crew, and the roster period, these block boundaries are included in the crew roster, which then will control the automatic pilot. Instead of starting with the first day of the roster period, and proceeding day by day until the last day of the roster has been checked for surplus manpower, the automatic pilot will consider one block at a time, and follow the directions specified by the block definition.

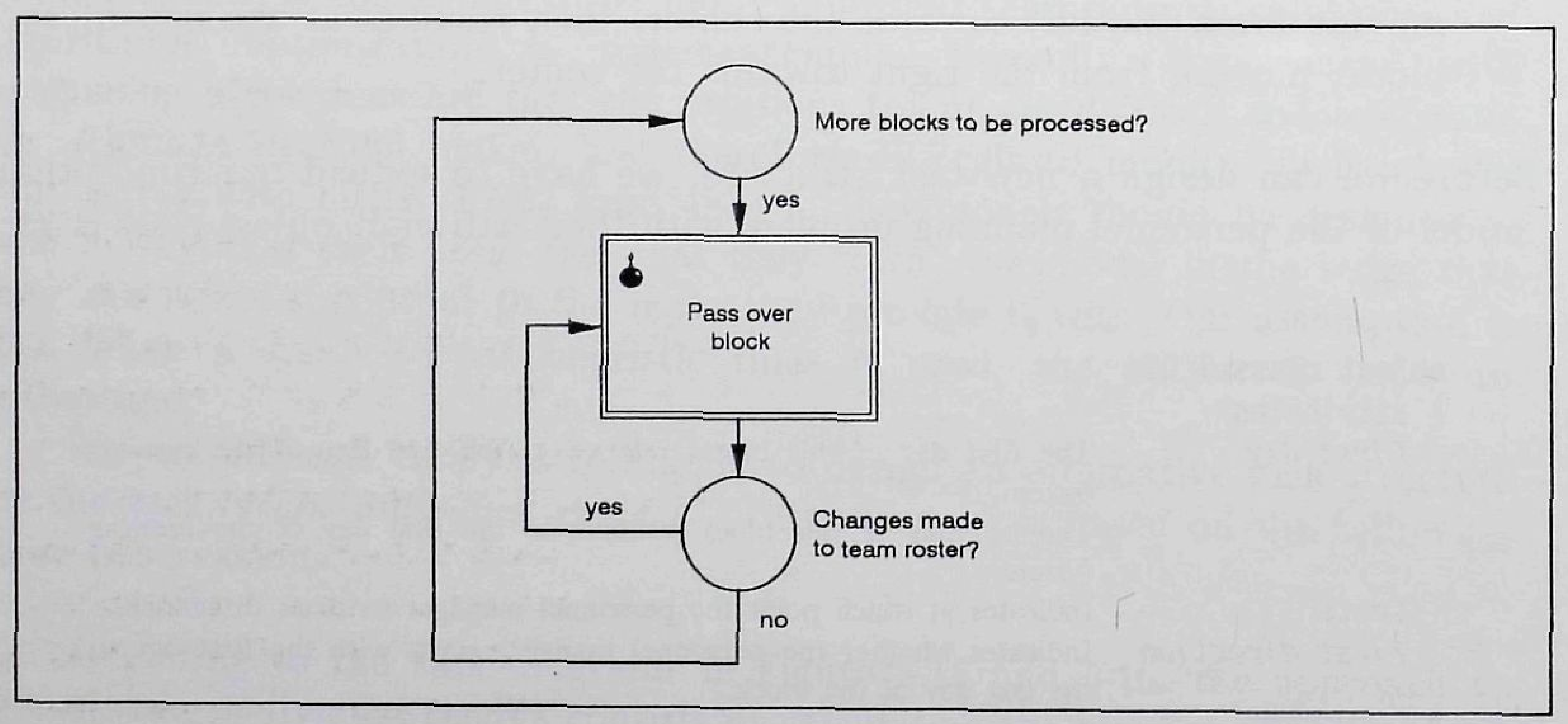

Fgure 5-21. The task "Make individual rosters for 1 function"
The task of making individual rosters for one particular function now means repeatedly looking for surplus and distributing shifts "off" for one block at a time, instead of for the entire roster period. Figure 5-21 shows the structure of this task. Observe that the task "Pass over block" is structured. The inner loop has been added to cope with ill-placed block centers. This is best explained by means of an example. Consider a production unit that is scheduled according to the crewing system of Figure 5-20 (including its block boundaries). Five mechanics are available. Two are needed on Sundays, five on Mondays, and four otherwise. There is no night shift on Saturdays. Figure 5-22 shows one week of the individual rosters of the five mechanics in various stages while "Pass over block" is performed. Initially, no shifts "off" have been allotted, so each mechanic is scheduled to work (pattern $a$ ). The block definition dictates that "Pass over block" processes days in the sequence Su-Mo-Tu-Sa-Fr-Th-We. The surplus on Sunday is 3 , so three mechanics get the day off. There is no surplus on Monday, but on Tuesday one shift can be removed. However, the fourth labor condition on page 102 forbids this. Figure 5-22(b) depicts the situation when the center of the block is reached. "Pass over block" proceeds in opposite direction, which results in pattern $c$.

\begin{tabular}{|c|c|c|c|}
\hline Mechanic & Su Mo Tu We Th Fr Sa & Su Mo Tu We Th Fr Sa & Su Mo Tu We Th Fr Sa \\
\hline $\begin{array}{l}0001 \\
0002 \\
0003 \\
0004 \\
0005\end{array}$ & $\begin{array}{llllll}N & N & N & N & N & N \\
N & N & N & N & N & N \\
N & - \\
N & N & N & N & N & N \\
N & N & N & N & N & N \\
N & N & N & N & N & N \\
N & N\end{array}$ & $\begin{array}{llllll}-N & N & N & N & N & - \\
-N & N & N & N & N & - \\
-N & N & N & N & N- \\
N & N & N & N & N & N- \\
N & N & N & N \\
N & N & N & N & N & N-\end{array}$ & 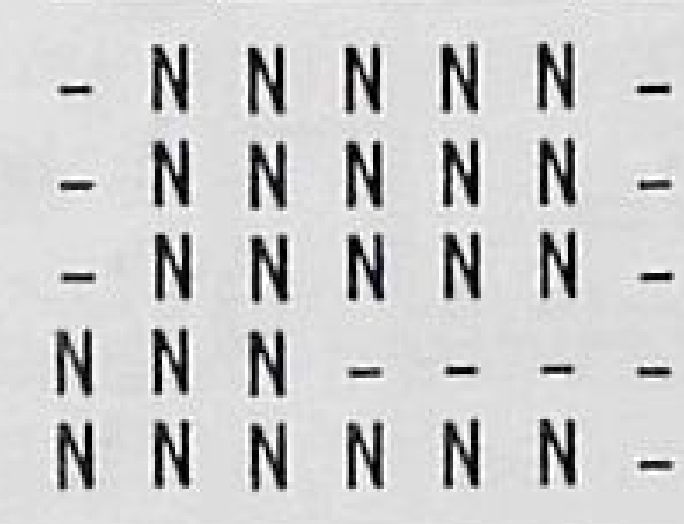 \\
\hline $\begin{array}{l}\text { Required: } \\
\text { Surplus: }\end{array}$ & $\begin{array}{llllllll}2 & 5 & 4 & 4 & 4 & 4 & 0 \\
3 & 0 & 1 & 1 & 1 & 1 & 0\end{array}$ & $\begin{array}{llllllll}2 & 5 & 4 & 4 & 4 & 4 & 0 \\
0 & 0 & 1 & 1 & 1 & 1 & 0\end{array}$ & $\begin{array}{lllllll}2 & 5 & 4 & 4 & 4 & 4 & 0 \\
0 & 0 & 1 & 0 & 0 & 0 & 0\end{array}$ \\
\hline
\end{tabular}

(a) (b)

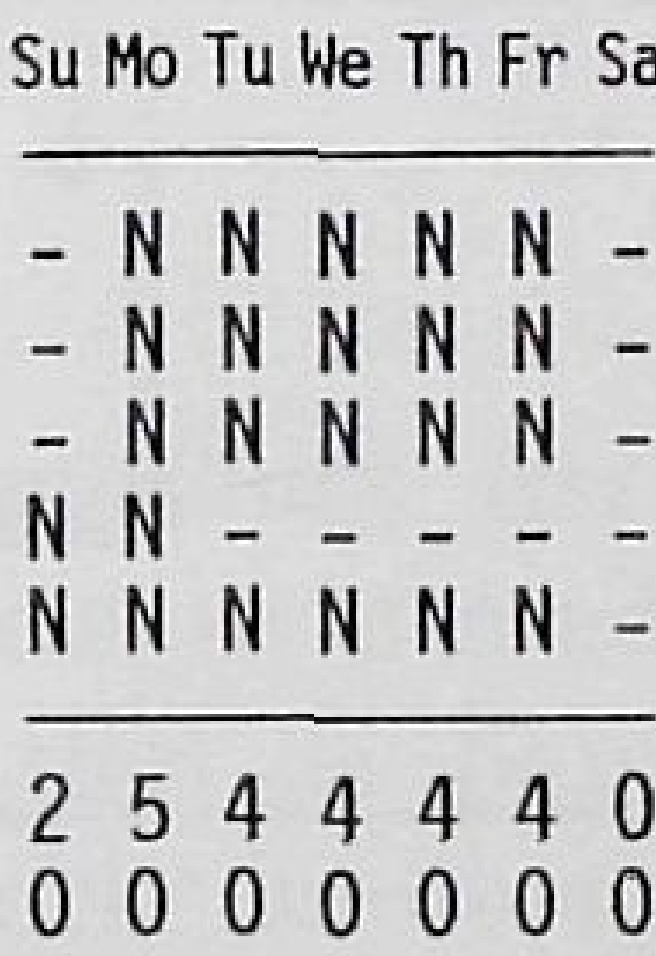

(d)
Figure 5-22. Two passes over a block of night shifts

The surplus on Tuesday can now be removed without violating any labor conditions, namely by giving mechanic 0004 the day off (pattern $d$ ). This requires a second pass. This second pass would not have been necessary when the block center had been placed between Monday and Tuesday. 


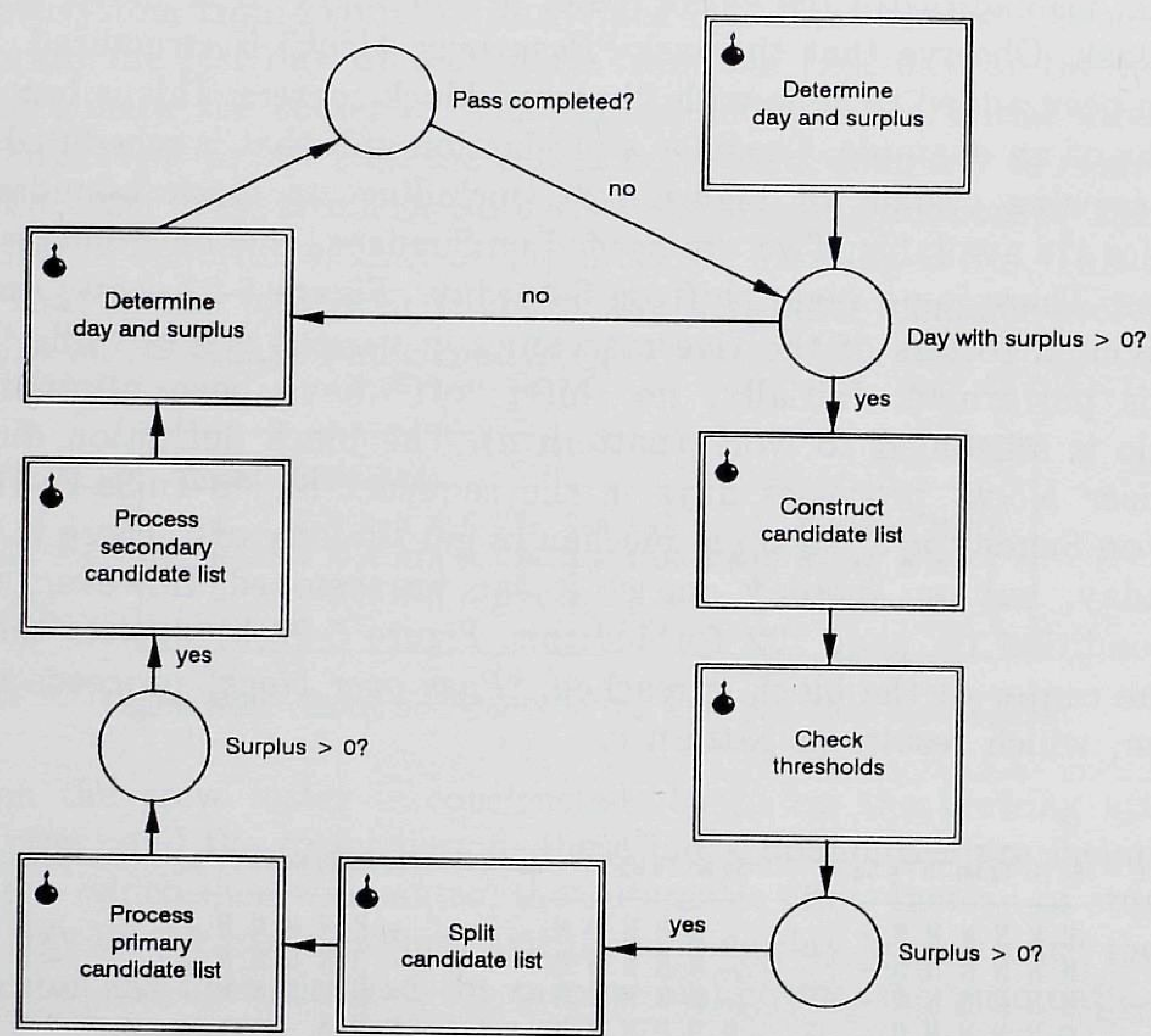

Fgure 5-23. The task "Pass over block"

Figure 5-23 shows the flow diagram of "Pass over block". Based on the block boundaries and initial direction, the first day is determined, and its surplus is calculated (see Figure 5-24). If there is surplus manpower, a list of "candidates" (workers that can be given the day off without violating the minimum number of shifts between two rest periods) is constructed. Candidates whose tallies exceed some pre-specified threshold value have top priority.

If there is more surplus than top priority candidates, the remaining candidate list is split in two. The primary list contains only those workers that already have a rest period directly preceding or following the current day, meaning that an extra day off will not break up a sequence of consecutive shifts (recall our third observation on page 117). The secondary candidate list contains the remaining workers.

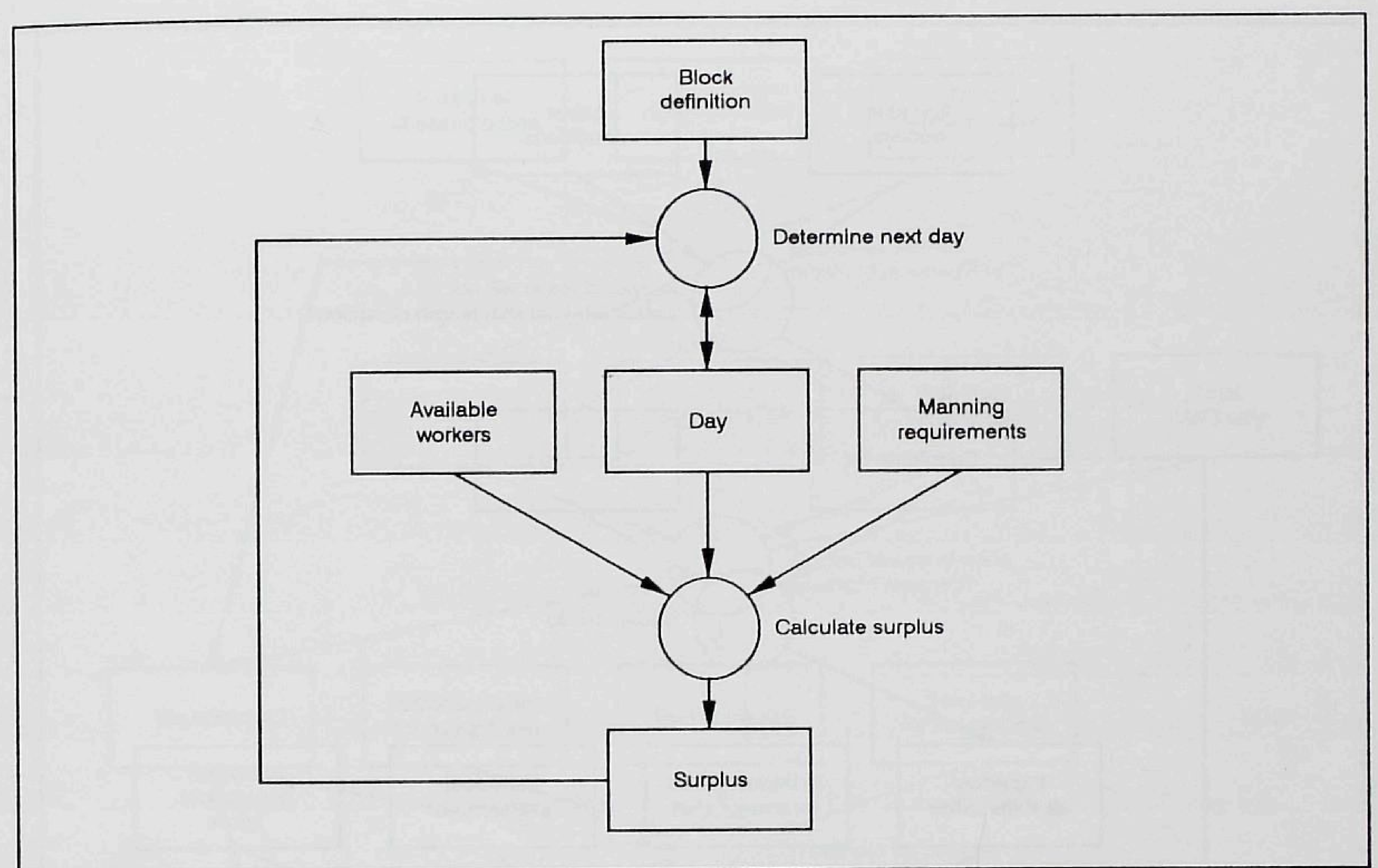

Fgure 5-24. The task "Determine day and surplus"

Figure 5-25 shows the decision structure of "Construct candidate list". Within this task, three decisions have been identified. The first decision is made for each available worker that still has to work on the day that is being rostered. The current team roster indicates who is scheduled to work, while the labor conditions specify the minimal number of consecutive shifts that has to be maintained. Once all candidates have been added to the list, tallies are updated. These tallies include for each worker the total number of shifts are worker has been scheduled for so far, the number of shifts of the kind currently being rostered (in Figure 5-22 this would be the night shift), the number of weekend shifts, and the number of weekends in which a worker has to work at least one shift. The grand totals over all workers are also calculated.

These tallies, individual preferences, and the workers' contractual hours affect the third decision: determine who is the best candidate. Since the surplus may be greater than one, the entire candidate list is sorted on individual preference, with the current shift tally/contractual hours ratio and the all shifts tally/contractual hours ratio as secondary and tertiary keys, respectively. 


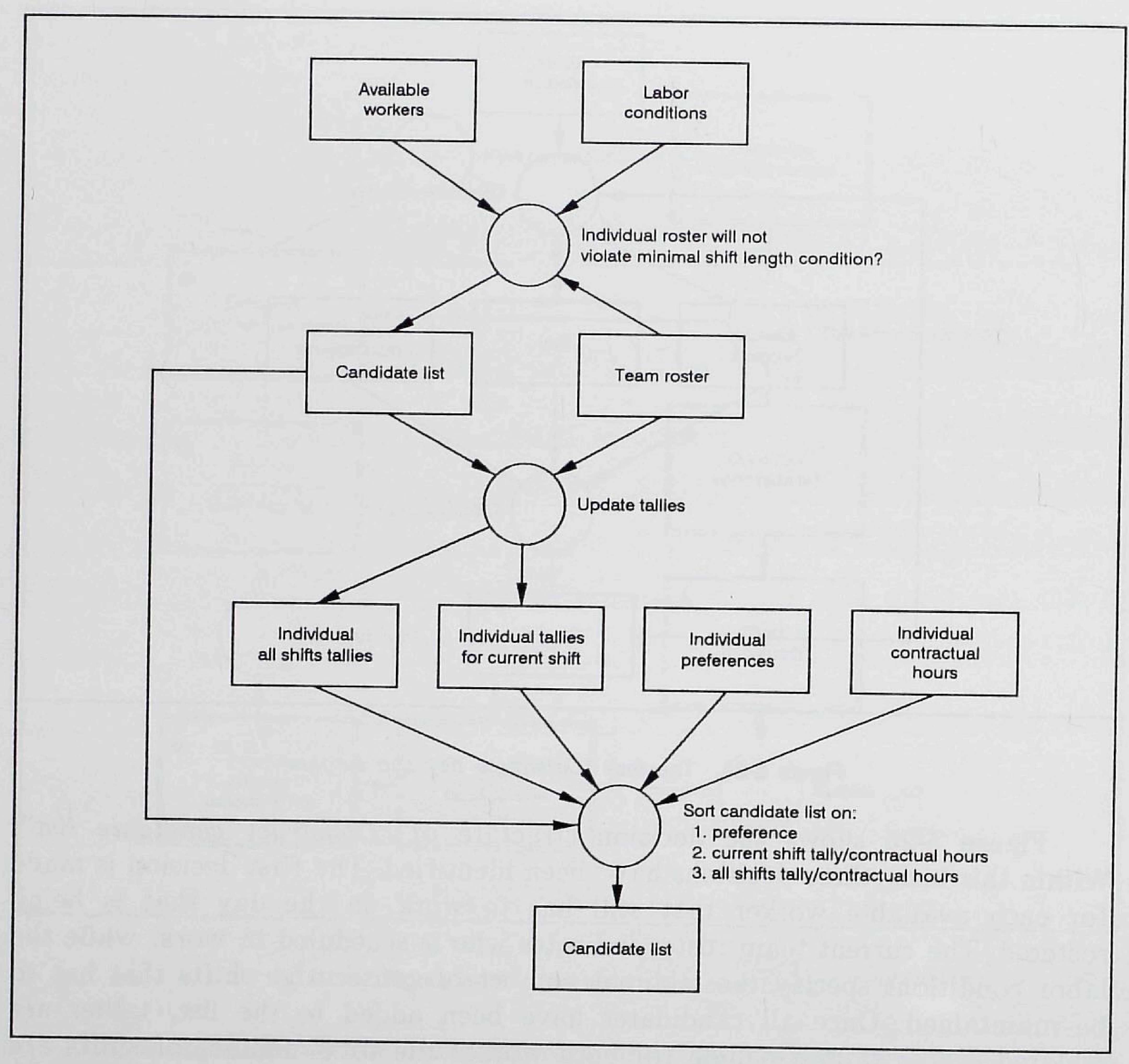

Fgure 5-25. The task "Construct candidate list"

These ratios play an important role in the "threshold checks". Basically, threshold values indicate the number of shifts (or weekends) that a worker may deviate from the "norm": the number of shifts that worker should be scheduled for to do exactly his share of work. For example, if a team includes five fulltime ( 40 contractual hours per week) workers and one part-time (20 hours per week) worker, and if the total number of night the norm for a full-time worker would be 20.2 shifts, and for the part-time worker 10.1. If the actual tallies are $21,18,19,21,20$, and 12 night shifts, and if the threshold value for this shift type has been set to 2 , the second worker obtains the "top priority" status because $|18-20.2|>2$.

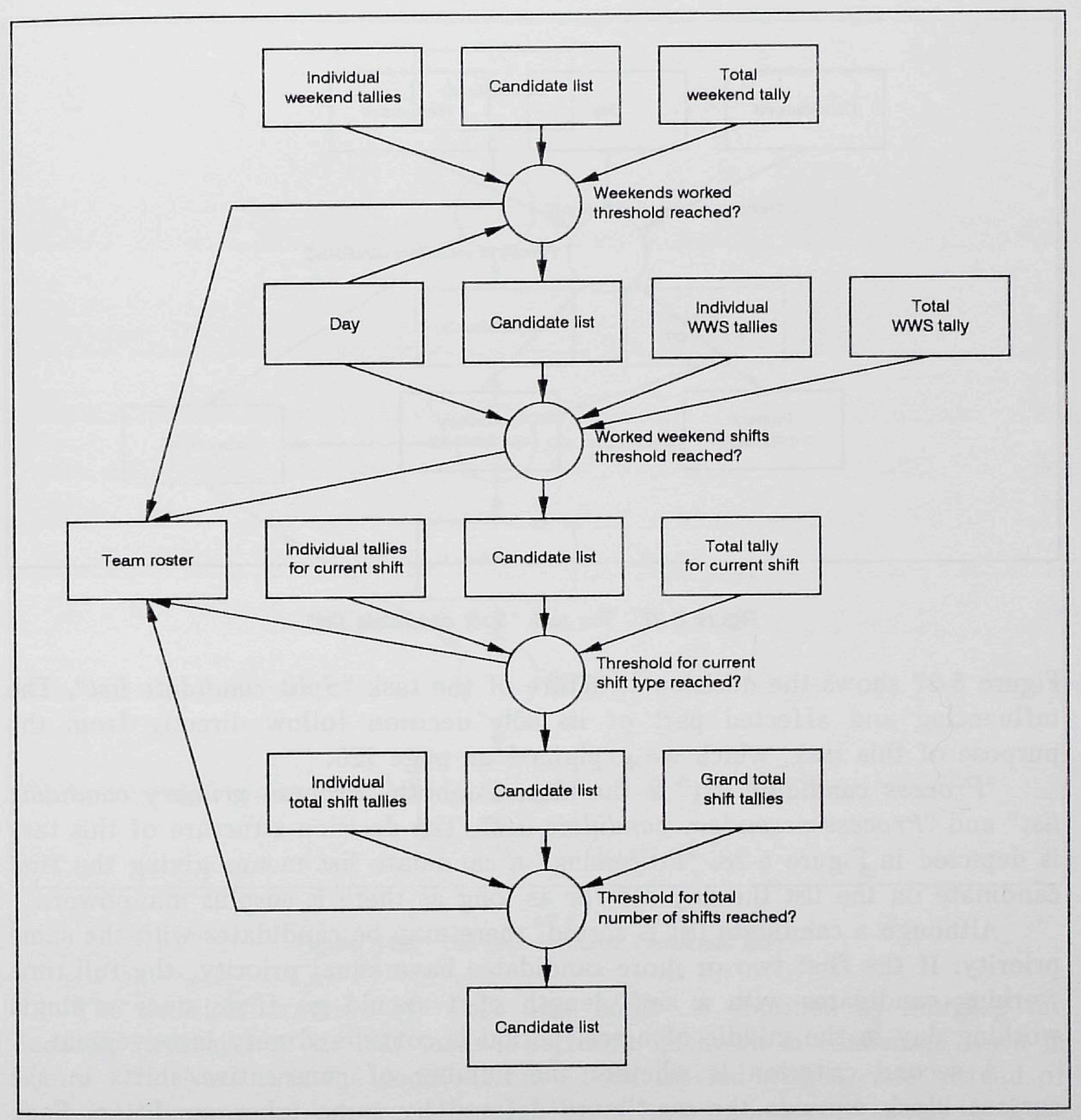

Floure 5-26. The task "Check thresholds"

Figure 5-26 shows the decision structure of "Check thresholds". The sequence of the four decisions is based on the fact that a fair distribution of weekends is appreciated most. The first two decisions only become "active" when "Day" is a Saturday or a Sunday. 


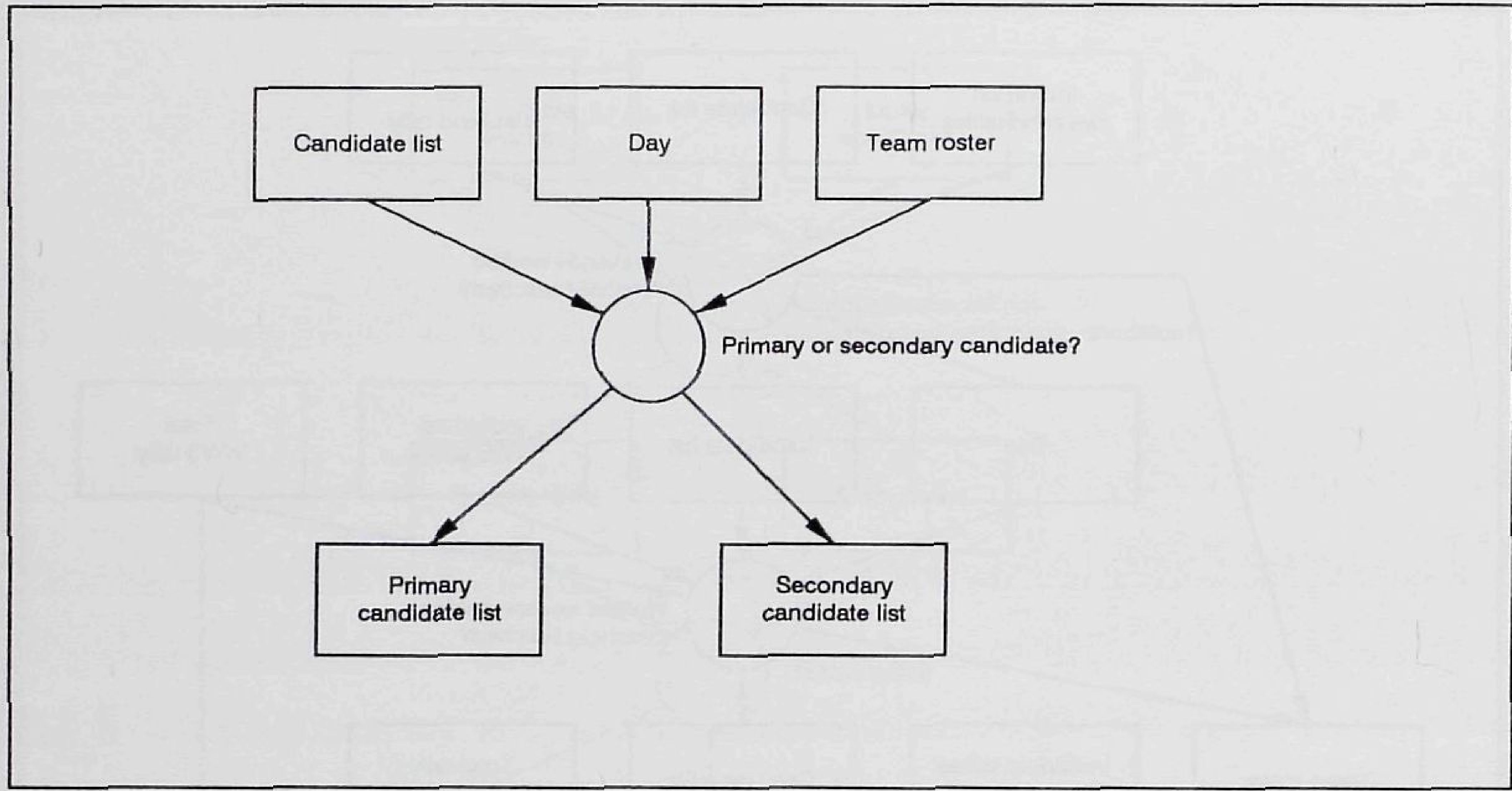

Fgure 5-27. The task "Split candidate list"

Figure 5-27 shows the decision structure of the task "Split candidate list". The influencing and affected part of its sole decision follow directly from the purpose of this task, which we explained on page 120 .

"Process candidate list" is the alias for both "Process primary candidate list" and "Process secondary candidate list". The decision structure of this task is depicted in Figure 5-28. "Processing" a candidate list means giving the first candidate on the list the day off for as long as there is surplus manpower.

Although a candidate list is sorted, there may be candidates with the same priority. If the first two or more candidates have equal priority, the full-time working candidates with a shift length of 1 should go first, since a single working day in the middle of a rest period is considered very inconvenient.

A second criterion is whether the number of consecutive shifts in the current block exceeds the maximum defined by some labor condition. Such maximum can be specified for each type of shift, as well as for shifts in general. The latter is useful when the shift cycle of the crewing system contains adjoining blocks, like for instance the blocks of morning and night shifts in Figure 5-20. If the maximum number of consecutive shifts of any type is set at 6 , the worker with roster "--MMMNNNN--" will ceteris paribus be preferred over the worker with roster "--MMMNNN---"

If neither rule applies, workers are released in the order in which they appear on the list until the surplus equals zero or the list is empty.

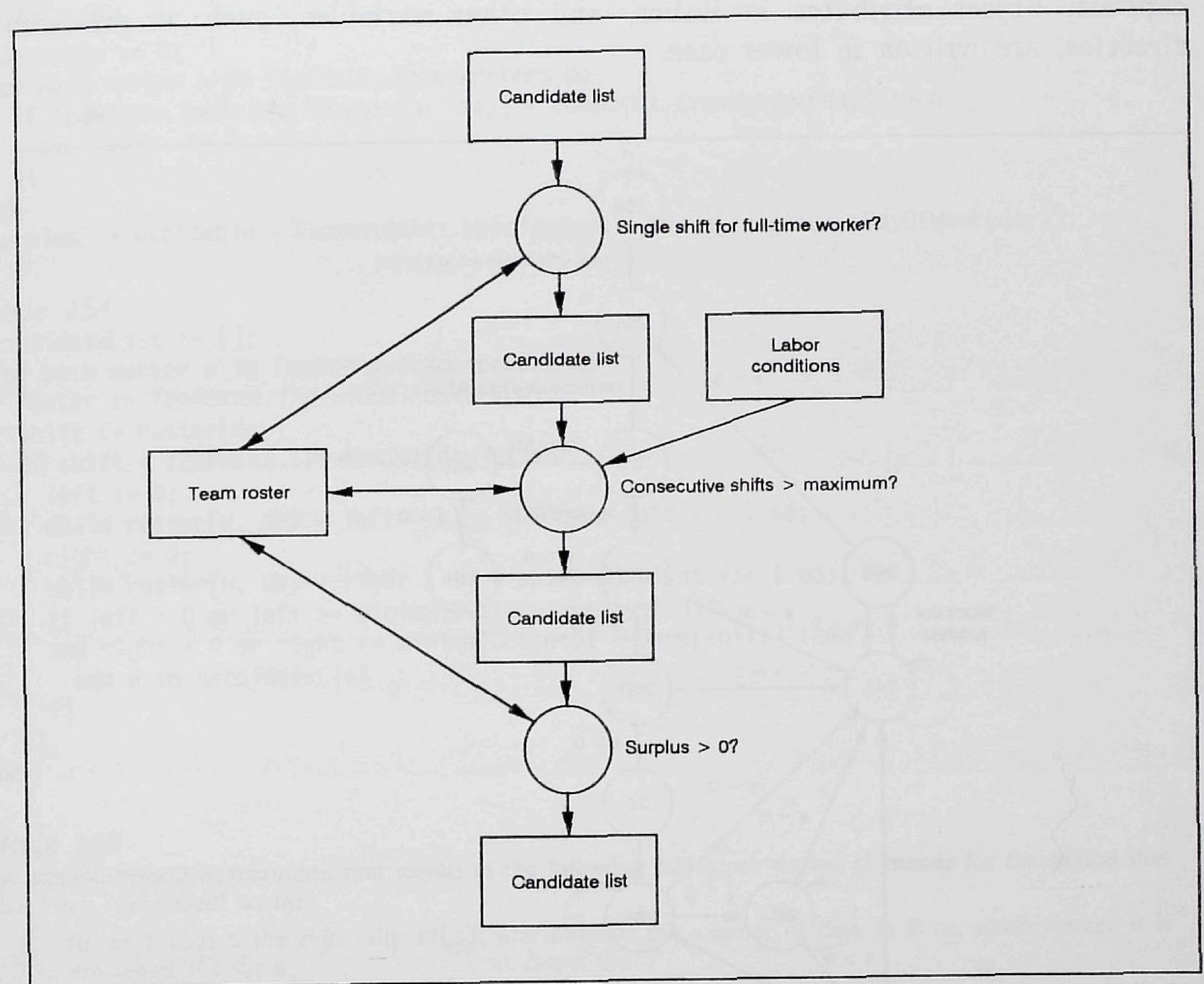

Flgure 5-28. The task "Process candidate list"

The decision structure of "Pass over block" is obtained by merging the decision structures of its leaf tasks. The result is shown in condensed form in Figure 5-29. Note that the decisions have been labeled with a code instead of their actual name for reasons of readability. The numbers correspond to those of the figures in this chapter. If a decision structure contains more than one decision, these decisions are enumerated A, B, C, etcetera from top to bottom. Thus, "Determine next day" in Figure 5-24 has code 24A, and "Calculate surplus" 24B.

Each of the thirteen decisions can be automated. The rules are stated on the next two pages in an Algol-like language. In case the reader is not familiar with the semantics of Algol, we refer to McGettrick (1978) for a detailed description. Object instances, such as BLock and TEAMROSTER are denoted in small 
capitals, object attributes in italics, and other variables, such as day and direction, are written in lower case.

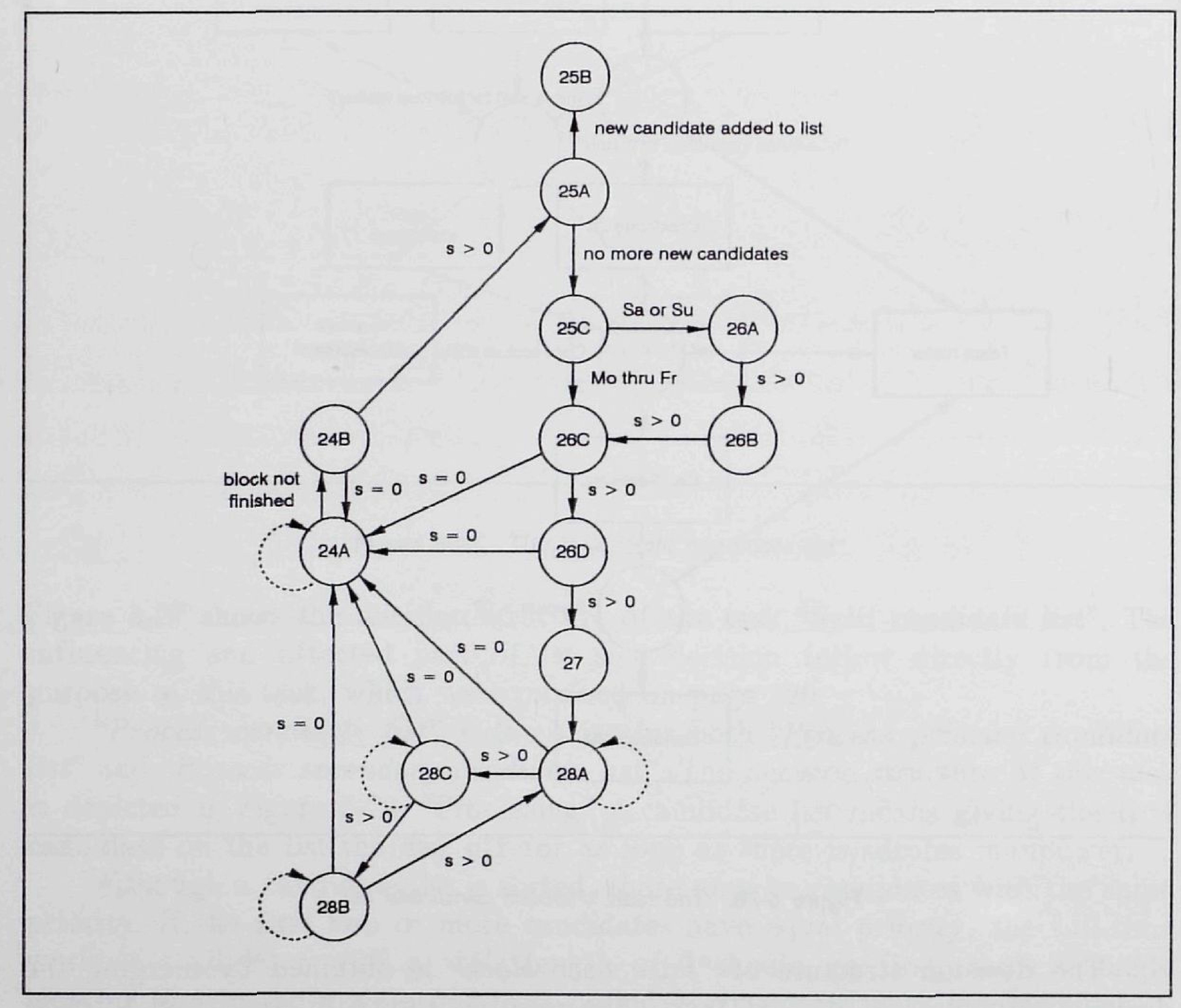

Foure 5-29. The condensed decision structure of "Pass over block"

\section{Rule $24 A$}

\section{direction := BLock.FirstDirection:}

if day = BLock. Center then

if direction = BLock. FirstDirection then

day := Block.LastDay;

fi

\section{else}

day $+:=$ direction

fi
Rule $24 B$

available $:=0$

for each worker $w$ in TEAMROSTER. Team. Workers do

If TEAMROSTER. Individua IRosters[w, day] = TEAMROSTER.CrewRoster[day] then available $+:=1$

$$
\text { od: }
$$

surplus := available - ProouctionUnit.ManningRequirements[function, DayOfWeek(day)];

\section{Rule $25 \mathrm{~A}$}

Rule $25 \mathrm{~A}$ candidatel ist $:=[]$;

for each worker $w$ in TEAMROSTER. Team. Workers do

roster := TEAMROSTER. Individua IRosters $[\mathrm{w}]$ :

shift $:=$ roster[day]

Shift := roster[day] if shift = TEAMROSTER.CrewRoster[day] then

left $:=0$;

while roster $[w$, day - left -1$]=$ shift do left $+:=1$ od;

right : $=0$;

whlle roster $[w$, day + right +1$]=$ shift do right $+:=1$ od;

if left $=0$ or left $>=$ minimalConsecutiveDays[shift]

and $r$ ight $=0$ or right $>=$ minimalConsecutiveDays[shift] then add $w$ to candidatel ist

Rule $25 B$

straightforward computation that results in the following tallies per worker (P stands for the period that has been "processed" so far):

1. for each shift $s$ the shift tally $s t[s]$, which equals the number of days in $P$ on which worker $w$ is not scheduled for S;

the "all shifts" tally ast $=\sum_{\xi} s t[\mathrm{~s}]$

3. the "weekends worked" tally wwt, which equals the number of weekends in $P$ in which worker $w$ is

scheduled for at least one shift,
the "worked weekend shifts" tally wwst, which equals the number of Saturdays and Sundays on which $\mathrm{W}$ will have to work.

Note that the abbreviations correspond to the tally-attributes of class WORKER. The total number of contractual hours tch, and the totals tst[s], tast, twwt, and twws of these tallies over all workers are also calculated.

\section{Rule $25 C$}

Since the number of candidates typically ranges from 1 to 10 , a simple insertion sort is most appropriate (Knuth 1973, page 73-157)

\section{Rule $26 \mathrm{~A}$}

for each worker $w$ in candidateL ist while surplus $>0$ do

if w.wwt - w.ContractualHours / tch * twwt > wwThreshold then

TEAMROSTER. Individua IRosters $[\mathrm{w}$, day $]:="-"$

surplus $-:=1$.

remove $w$ from candidatelist

( 


\section{Rule $26 B$}

for each worker $w$ in candidatel ist while surplus $>0$ do

If w.wwst - w.ContractualHours / tch * twwst $>$ wws Threshold the

TEAMROSTER. Individua IRosters[w, day] := "-"

surplus $-:=1$;

fi

Rule $26 C$

for each worker $w$ in candidateL ist while surplus $>0$ do

if w.st[s] - w.ContractualHours / tch *tst[s] > shiftThreshold[s] then TEAMROSTER. Individua IRosters $[\mathrm{w}$, day $]:="-"$

surplus $-:=1$;

fi

Rule $26 D$

for each worker $w$ in candidatel ist while surplus $>0$ do

if w.tst - w.ContractualHours / tch * tst > totalShiftThreshold then

TEAMROSTER. Individua IRosters $[\mathrm{w}$, day $]:="$ " "

surplus $-:=1$;

remove $w$ from candidatel is

Rule 27

primaryCandidates $:=$ secondaryCandidates $:=[]$

for each worker $w$ in candidatel ist do

roster := TEAMROSTER. IndividualRosters [w]:

If roster[day -1$]=$ " " or roster $[$ day +1$]=$ "-" then

add $w$ to primaryCandidates

else

add $w$ to secondaryCandidates

Rule $28 \mathrm{~A}$

for each worker $w$ in candidatel ist while surplus $>0$ do

roster := TEAMROSTER. IndividualRosters[w];

if $\mathrm{w}$. ContractualHours $>=38$ and roster[day -1$]="$ " " and roster $[$ day +1$]="$ " then

roster[w, day $]:="-"$;

surplus $-:=1$;

fi

od

\section{Rule $28 B$}

for each worker $w$ in candidatel ist while surplus $>0$ do

roster := TEAMROSTER. IndividualRosters[W];

shift $:=$ roster [day]

left $:=0$;

while roster $[w$, day - left -1$]=$ shift do left $+:=1$ od;

right : $=0$;

while roster $[w$, day + right +1$]=$ shift do right $+:=1$ od:

If left $>$ maximumConsecutiveDays[shift] or right $>$ maximumConsecutiveDays[shift] then roster $[w$, day $]:="-"$;

surplus $-:=1$;

remove $w$ from candidatelist

Rule $28 \mathrm{C}$

for each worker $w$ in candidatel ist while surplus $>0$ do

TEAMROSTER. Individua IRosters $[\mathrm{w}$, day $]:="$ " "

surplus $-:=1$ :

remove $w$ from candidatel ist

These decision rules and the simple demon logic associated with the edges in Figure 5-29 have been translated into Turbo Pascal ${ }^{\mathrm{TM}}$ (Borland 1987), and are part of the software in directory $\backslash \mathrm{RP}$ on the diskette that came with this thesis.

We conclude this section with some evaluating remarks with respect to the roster worksheet and the automatic pilot function. The choice of items is adopted from Keen (1981).

Increase of the number of alternatives examined

The support component we developed for "Plan personnel" scores highly on this criterion. The automatic calculation and immediate display of tallies stimulates the personnel planner to patch rosters more thoroughly. Moreover, we found that the automatic pilot function turns out to be the perfect tool for testing the feasibility of different crewing systems or team compositions. The quality of individual rosters largely depends on the "mix" of full-time and parttime workers in a team, but this relation can be complex. The calculations for determining team size (see page 101) provide insufficient information. Since the automatic pilot shows good performance (it generates one-year individual rosters for 10 workers in less than 5 seconds) the personnel planner can experiment with different team compositions simply by repeatedly changing the contents of one table, activating the automatic pilot, and judging the quality of the results. 
Fast response to unexpected situations

A good example is the case where the unions vetoed a roster proposal because it offered insufficient compensation for certain holidays. Normally this would have meant a delay of more than two weeks, not only because of re-planning but also because the unions require time to check the new proposal and it calculations. With the aid of computerized support, a new proposal that provided adequate compensation was available almost immediately. It was accepted that same day, since the correctness of the tallies on the computer print-out was not questioned.

Ability to carry out ad hoc analysis

The roster worksheet supports the planner during all kinds of analyses. Testing the feasibility of specific crewing systems, determination of relative work loads for different team sizes, and calculation of the financial consequences of inconvenience bonuses are good examples.

New insights and learning

We found that the systematic rostering approach described in this chapter is an eye-opener to less experienced planners. The possibility to experiment with different crewing systems leads to new insights even for experienced planners.

\section{Control}

The direct "translation" of individual rosters in terms of working hours and inconvenience bonuses facilitates cost control

\section{Cost savings}

Cost savings result mainly from a more efficient use of the human resource. Part-time workers make a work force more flexible. The disadvantage that they complicate the rostering process has largely been eliminated.

Better decisions

The automatic pilot function does not yield perfect rosters. However, "manual" patching takes place the same way as before, except that it is based on more accurate information. The eventual result is judged by the same or higher standards, meaning that the quality of the rosters (in our opinion the only measure for the quality of the decisions) is greater or equal to that of completely hand-made rosters.

\section{Time savings}

Time savings have been measured for the task of making a team roster in the situation described in Section 5.3. The values in Figure 5-30 have been calculated by subtracting the actual time needed to perform a leaf task from the norm values in Figure 5-17. We use these theoretical values because data on the enly at the agoregate unstructured, manual rostering process could be to make a team roster by hand could be measured. This total (almost two working days) closely approximates the theoretical value, which ranges from 13.5 to 15 hours.

\begin{tabular}{|l|c|}
\hline Leaf task & Actual time savings \\
\hline Determine appropriate crewing system & none \\
Define roster period & none \\
Compose team & 5 minutes \\
Process notifications of absence & 3 minutes \\
Determine function groups & 2.5 minutes \\
Determine relative work loads & 2.5 minutes \\
Compute \# of shifts "off" workers are entitled to & 7.5 minutes \\
Distribute these evenly over roster period & 8.5 hours \\
Patch "offending" roster & 1.5 hours \\
\hline Total & 10.3 hours \\
\hline
\end{tabular}

Fgure 5-30. Actual time savings for "Plan personnel" with worksheet and automatic pilo

There are several explanations for the differences in estimated and actual time savings. Firstly, it is tempting to spend a lot of time "tuning" the automatic pilot function. Since the quality of the rosters it produces rises rapidly to some $90 \%$, users of the automatic pilot tend to postpone the stage of manual patching too long, trying to reach some optimum the algorithm is incapable of finding. A second reason is that as the rostering process becomes easier, the planner's ambition level rises. Much of the time that is gained is spent on efforts to accommodate even more personal preferences, or to get the fluctuation of the average inconvenience below $0.1 \%$. In other words, the tradeoff between time gain and roster quality is often made in favor of the latter.

\subsection{SUMMARY AND CONCLUSIONS}

In this chapter we reported on our analysis of the problem-solving process of an individual decision maker. We presented a detailed conceptual model of a personnel manager's problem situation, and gave an example of how this conceptual model can be "translated" into an empirical model. We demonstrated how the results of task analysis can be used to estimate the pay-off of supporting a particular task.

Our conclusions fall into two categories. Those in the first category relate to our approach to problem solving in general, based on our experiences with object- 
oriented modeling and our task analysis technique. Those in the second category relate to our solution of the problem of planning shiftwork in particular.

\section{The problem-solving approach}

Our first and most important conclusion is that we can indeed describe a specific problem situation using our method for task analysis.

A second conclusion is that even the limited form of simulation used in this case (the empirical model does little more than compute tallies) provided valuable information in a manner that is easy to follow even by non-experts.

Finally, we found that working with decision structures, both analytically and by experimenting with decision rules and demon logic, is a practical and stimulating means to develop and test heuristic programmes.

\section{The shiftwork planning problem}

We have shown that shiftwork planning can be structured to a large degree, and that adequate computer support can be developed. However, we did not address the "patching" problem. Further task analysis of "Patch 'offending' roster", based on observation of very experienced planners, might lead to a patching programme that is both time-efficient and effective. Recently, Verbraeck (1989) published his results of an "analysis-through-observation" of the task of making teaching schedules for schools. His findings suggests that such patching programmes can indeed be found.

\section{GROUP PROBLEM SOLVING SUPPORT}

\section{THE CASE OF INTERNATIONAL TRANSFER PRICING}

What is a cynic?

A man who knows the price of everything and the value of nothing. - Oscar Wilde

\subsection{INTRODUCTION}

Our second case study focuses on group problem solving. We wish to investigate whether the process of a group of decision makers working together on one particular problem can be described, analyzed, and possibly improved using the approach from Chapter 3 .

The specific problem we shall be looking at is choosing the best means for fund transfer between parent and subsidiary companies of a multinational company in the pharmaceutical industry, with an emphasis on the setting of transfer prices (being one of the means for fund transfer).

The analysis presented in this chapter is the result of a joint research effort of Van der Ven and the author of this thesis. Since our main interest lies in testing and improving our task analysis technique, our description of the problem will be relatively brief and slightly simplified, while we cover the task analysis in much more detail. For a detailed problem specification and extensive background information we refer to Sol and Van der Ven (1988) and Van der Ven $(1989 a, b)$.

At the basis of the fund transfer problem lie the financial ties between parent and subsidiary companies within the same multinational. The direction in which funds are transferred mainly depends on the liquidity situation of the subsidiary company. An excess of funds resulting from operations is called a liquidity surplus. Similarly, liquidity shortage is defined as a lack of funds. Although this is definitely not a general rule (Van der Ven 1989a, page 34), we assume in this chapter that liquidity surplus calls for a fund transfer from subsidiary to parent company, and that liquidity shortage requires a fund transfer from parent to subsidiary company.

A liquidity shortage can of course be balanced by a decrease in cost or an increase in sales by means of a temporary rebate, and converse actions will affect a liquidity surplus. All such alternatives - which primarily have an impact on the multinational's environment - are called "operational alternatives". Alternatives that cause a shift in activities (and thereby costs) within the multinational, for example shifting production or packaging activities from one group company to another, without affecting its environment are called "relocation alternatives". Finally, those alternatives that only cause an internal flow of funds are called "financing alternatives". 
Relocation and financing are secondary activities within the organization. The focus is on the operational activities. In this chapter, however, we shall look at the financing alternatives only. This alone is already a very complex matter. One complicating factor is that there are various instruments for fund transfer from parent to subsidiary company or vice versa. These can be grouped into six categories:

\section{Transfer prices}

Prices set for the transfer of goods or services between two companies belonging to the same multinational are called "transfer prices". In this case study we only consider the transfer of goods from a parent company (the production center and supplier) to one of its subsidiary companies. In this context, transfer price payments are the payments from a subsidiary company to the parent company.

\section{Royalty}

Royalty payments are payments made by the subsidiary company to the parent company in exchange for the right to use patents, propriety trade marks, or other intangible assets from the parent company.

\section{Dividend/share capital}

Dividend is the part of a company's profit that is distributed among the shareholders in proportion to their share of ownership. Since the parent company is sole shareholder, a dividend payment constitutes a fund transfer from subsidiary to parent company. Similarly, a share capital payment (the parent company investing more money in its subsidiary company) constitutes a fund transfer in opposite direction.

\section{Current account}

The "current account" is the ongoing trading-debt position of the subsidiary company due to the purchase of goods. By decreasing the subsidiary company's payment term, debts have to be paid off earlier. Basically, this constitutes a fund transfer from subsidiary to parent company. By increasing the subsidiary company's payment term, a fund transfer in opposite direction is effectuated.

\section{Intra-company loan}

A loan is a sum of money, borrowed at interest, for a period of time. The subsidiary company can lend out to the parent company and vice versa.

\section{Cost transfer}

A cost transfer is a payment from the parent company to the subsidiary company (or vice versa) as a compensation for special expenses.
Some ways of transferring funds, for example a share capital increase of an intra-company loan, affect only the liquidity situation of the subsidiary company. Others, such as a royalty or transfer price increase, have an impact on both the liquidity situation and the profitability of the subsidiary company. Costs of the various ways to transfer funds are also of importance. Tax and duty rates may significantly affect these costs.

Compensating the liquidity surplus or shortage of a subsidiary company is a multi-disciplinary process, since it has an impact not only in the financing area, but also in the fiscal and commercial area. In general, the following organizational units are represented in the group of decision makers: local management, central management, price coordination, fiscal affairs, and financial affairs. The people involved usually are chief specialist, or general managers - just under the top level. This group of decision makers is presumed to act as a team (Van der Ven 1989a, page 43). Consequently, the type of group problem solving discussed in this chapter is one of "expertise sharing".

The problem can be summarized as follows: a group of decision makers has to determine the amount of funds to be transferred from a subsidiary company to the parent company or vice versa, the means by which this transfer should take place (often a combination of several of the mentioned financing alternatives), and - if the transfer price alternative is one of these means - the specific products that have to be re-priced, and the new prices, all within the constraints imposed by external parties (government, customs, health care authorities, etcetera).

We decided to use the problem of international transfer pricing in the pharmaceutical industry as a case study because it met the following demands:

\section{Relevance}

Again, we were looking for a problem of present interest. Transfer pricing - particularly within the pharmaceutical industry, where high centralized cost must be covered by international intra-company sales - meets this requirement. Its relevance is pointed out by for example Verlage (1975), Plasschaert (1979), Nowotny (1987), and Von Grebmer (1987).

\section{Demarcation}

The specific problem absolutely had to require more than one decision maker, and preferably include team setting and consensus aspects. The fund transfer problem met both requirements. Moreover, the fact that the fund transfer problem occurs at the tactical/strategical level rather than at the operational level provided an opportunity to test whether our task analysis technique can be applied to problem-solving processes on a higher level of management. 
Complexity

Firstly, the problem had to be so complex as to require the expertise of more than one decision maker. Secondly, it should surpass these decision makers's cognitive abilities, meaning that their problem-solving process had to be characterized by a satisficing approach. The group meeting described by Van der Ven (1989a, page 73-78) clearly demonstrates the bounded rationality of the participants.

\section{Structuredness}

Similar to our previous case, the problem situation had to be ill-structured, meaning that no formalization of the decision process had to have taken place so far. As will be shown in Section 6.4, the transfer pricing problem met this condition.

\section{Field test}

The problem had to be "real life", and had to and recur on a more or less regular basis, thus providing the possibility to check the task analysis results against a new situation. Although the frequency of the group meetings for transfer pricing decisions is much lower than that of the shiftwork planning sessions analyzed in the previous chapter, the situation at the host company (which we shall refer to as "Europhar") provided sufficient opportunity to compare the situation before and after task analysis had been performed.

In all, we consider subsidiary company financing a fit subject for a case study on group problem solving. Using the problem-solving approach from Chapter 3 we shall analyze this problem in depth. In the following two sections we describe the activities of problem conceptualization and specification, while Section 6.4 reports on our task analysis. In Section 6.5 we describe how the various subtasks of subsidiary company financing can be supported by means of information technology. Finally, we summarize this case study in Section 6.6 and present our main conclusions.

\subsection{CONCEPTUALIZATION}

Like we did in Section 5.2, we start out with identifying the object classes in terms of which the international transfer pricing problem can be described. Again, we define object classes in terms of attributes and actions. New in this section is the use of subclasses and inheritance. The obvious way to express that parent company and subsidiary companies are companies with a number of common traits, is to define one object class COMPANY, and subsequently identify a subclass of COMPANY for each of the two company types. Hence the following definitions:

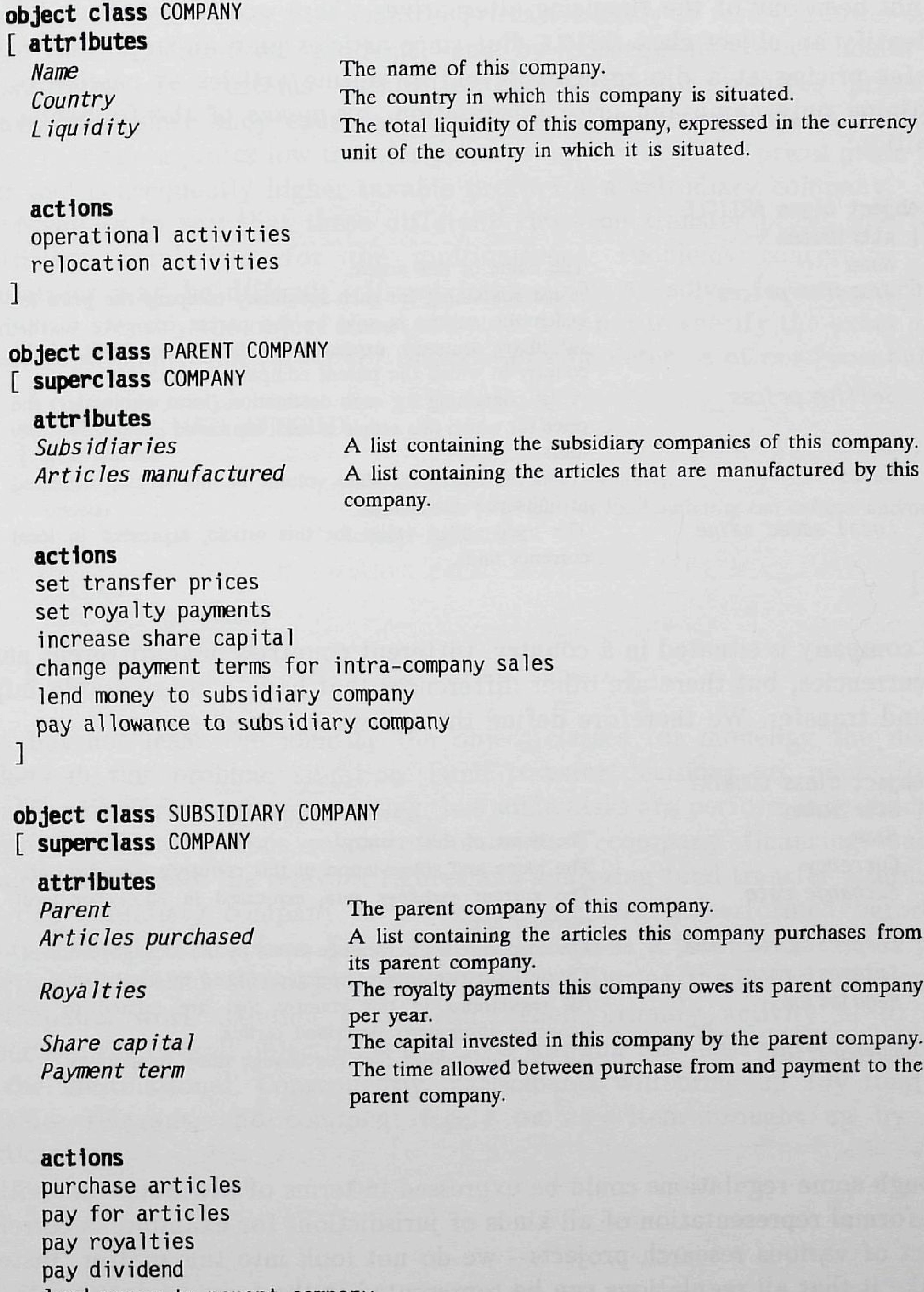

actions

operational activities$$
\text { relocation activities }
$$

\section{object class PARENT COMPANY}

[ superclass COMPANY

attributes

Subsidiaries

Articles manufactured

A list containing the subsidiary companies of this company. A list containing the articles that are manufactured by this company.

actions

set transfer prices

set royalty payments

increase share capita

increase share capital

for intra-company sales

lend money to subsidiary company

]

\section{object class SUBSIDIARY COMPANY}

[ superclass COMPANY

\section{attributes}

Parent

Articles purchased

Royalties

Share capital

actions

purchase articles

pay for articles

pay royalties

pay dividend 
The action lists of the company classes include operational, relocation, and financing activities. Since we are interested in activities in the financing area only, those in the other areas are not represented in detail. If transfer pricing had not been one of the financing alternatives, there would have been no need to identify an object class ARTICLE. But since articles play an important role in transfer pricing at a disaggregate level, we define articles as passive objects containing only name and price information, by means of the following class definition:

\section{object class ARTICLE \\ [ attributes}

Transfer prices

Selling prices

Sales

Local added value

Each company is situated in a country. Different countries have different names and currencies, but there are other differences that have a considerable impact on fund transfer. We therefore define the following object class:

\section{[ attributes \\ Name}

object class COUNTRY

Currency

\section{Corporate tax rate}

Interest rates

Regulations

]
The name of this article.

A list containing for each subsidiary company the price for which this article is sold by the parent company to that country in which the parent company is situated.

A list containing for each destination (local wholesaler) the units.

A list containing the sales volume of this article, expressed in units per destination.

added value for this article, expressed in local currency units. price for which this article is sold, expressed in losaler) the

\section{The name of this country.}

The name and abbreviation of this country's currency unit. The current exchange rate, expressed in NLG per local currency unit.

The corate tax percentage levied by the local government. The interest percentages charged/offered by local banks. All regulations in this country that are related to the financing alternatives described earlier.

All local authorities that can change these regulations.

Although some regulations could be expressed in terms of attributes and actions - the formal representation of all kinds of jurisdiction, for example, is currently subject of various research projects - we do not look into this matter. Instead, we take it that all regulations can be represented in the form of documents. We shall return to this matter in Section 6.5.

The fact that various local authorities are involved considerably complicates the transfer pricing problem, since the interests of different authorities are often diametrically opposed. The national health organizations, for example, wish to contain health care costs. Low transfer prices are likely to result in lower selling prices of pharmaceutical products, and by consequence lower health care expenditures. To customs authorities, however, low transfer prices are unfavorable, since they cause a decrease in revenue from duties. But fiscal authorities again prefer low transfer prices, since low transfer prices mean lower costs and consequently higher taxable profit for a subsidiary company.

Needless to say that these different views on transfer prices may cause conflicting regulations for the multinational. Problems concerning these regulations may be difficult - if not impossible - to solve. In our conceptua model we identify the object class LOCAL AUTHORITY, not to specify the exact nature of these problems, but rather for the sake of completeness of our "vocabulary".

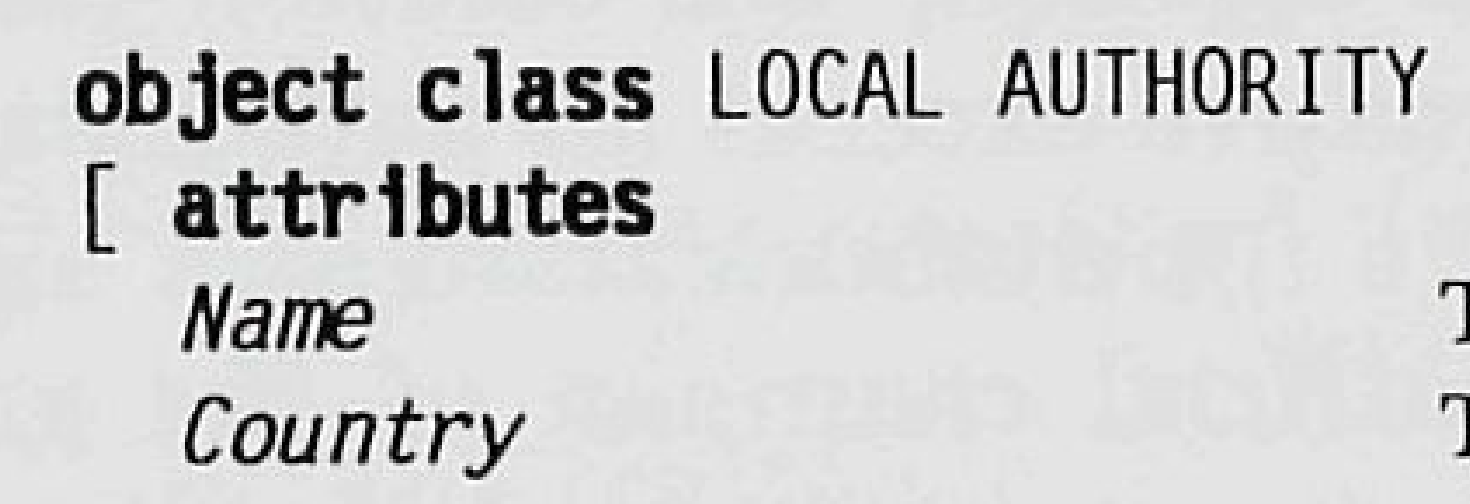

Country

The name of this local authority.

The country in which this local authority can enforce and/or change regulations.

\section{actions}

enforce regulations

change regulations

Last but not least, we identify the object classes for modeling the decision makers in this problem situation. Fund transfer decisions are made for the greater part during a group meeting, but some tasks are performed individually. These individual actions related to subsidiary company financing, such as bringing up-to-date the relevant figures and analyzing fund transfer alternatives for one subsidiary company in particular, are largely performed before the group meeting takes place. Since each individual is a participant in the group meeting, we define the object class PARTICIPANT. During the group meeting, the participants work together as a team. Their primary activity is to share expertise, the overall objective is to find a solution that best serves the interest of the multinational. Consequently, participants will bring up any item they consider relevant, and comment freely on any item brought up by other participants. 


object class PARTICIPANT
$\begin{array}{ll}\text { [ attributes } & \text { The name of this participant. } \\ \text { Name } & \text { The specific company within the multinational for which this participant } \\ \text { Company } & \text { works. } \\ \text { Function } & \text { The function this participant holds within that company. }\end{array}$

actions

actualize budget figures

analyze alternative

bring up item considered relevant

comment on items brought up by other participants

One of the participants has the role of "authorizer", meaning that only when he attaches his formal agreement to a proposed solution, this solution can be implemented. It is possible that a particular alternative exceeds his authorization level, in which case he will submit this alternative for authorization at a higher management level. The authorizer is also the official chairman of the group meeting. Moreover, if the group does not reach consensus, it is the authorizer who cuts the Gordian knot. Since the authorizer participates in the group meeting but can perform certain actions the other participants cannot, it is natural to define AUTHORIZER as a subclass of PARTICIPANT.

\section{object class AUTHORIZER \\ [ superclass PARTICIPANT \\ attributes}

Authorization level

\section{actions}

\section{chair group meeting}

break impasse

authorize alternative

]

These object classes leave us with three "loose ends": the actions of PARTICIPANT and AUTHORIZER make reference to budget figures, alternatives, and items, but none of these has been identified as an object class. In terms of the PSSE we described in Chapter 4, each can be modeled as a subclass of INFORMATION. The following definition of BUDGET is not meant to be complete, but rather to give a general notion of the kind of information it contains.

\section{object class BUDGE}

[ attributes

Short-term profit and loss Monthly figures as expected for the coming year. Balance sheet

]

Each fund transfer alternative falling in one of the six categories described on page 134 can be modeled as an instance of the information subclass ALTERNATIVE defined by:

\section{object class ALTERNATIVE \\ [ attributes \\ Transfer type}

Background information

Maximal remittance

Amount

Financial impact

Since the items that are brought up during a group meeting can vary widely in contents, we specify an item using only two attributes:

\section{object class BACKGROUND INFORMATION ITEM}

\section{attributes}

Area

Contents

The area of activities to which this item relates.

]

Having identified the classes of all objects that are involved in transfer pricing, the next step is to apply the information paradigm in order to determine the actual boundaries of the problem situation. If we view the transfer pricing problem situation from the macro-perspective, the RS-component of the IS/RScombination consists of the parent company and its subsidiary companies. The group of participants constitutes the IS-component. Countries and their local authorities belong to the IS/RS-combination's environment. Articles (and thereby all attributes and actions that relate to articles, such as Articles manufactured and 
purchase articles) are not considered at the macro-level, since they are involved only indirectly, namely through actions performed by companies. The IS (the group of participants, which includes managers from both parent and subsidiary company) controls the RS by inducing the parent company and/or the subsidiary company to perform one or several of the actions specified by the class definitions on page 137.

However, if the transfer price alternative is one of the chosen means for fund transfer, the participants must decide not only the amount of money that has to be transferred through a transfer price increase or decrease, but also which specific articles can have their price changed. In other words, ARTICLE must be part of the RS. For this reason we must increase the level of detail by applying the recursion principle to the macro-level RS-component. Doing so, we can identify various IS/RS-combinations within the original RS.

Our objective is to analyze the subsidiary company financing problem, and to improve the programme according to which it currently is solved. Since we are primarily interested in what takes place in the financing area, the obvious choice is to view operational, relocation, and financing activities as separate IS/RS-combinations. We shall consider the financing activities only. The attributes and actions that relate to the other areas can be considered as part of this IS/RS-combination's environment.

The boundaries of the problem situation that we shall be investigating in the remainder of this chapter are as is indicated in Figure 6-1.

\begin{tabular}{|l|c|}
\hline Object class & is part of the \\
\hline PARENT COMPANY & RS \\
SUBSIDIARY COMPANY & RS \\
ARTICLE & RS \\
PARTICIPANT & IS \\
AUTHORIZER & IS \\
BUDGET & IS \\
ALTERNATIVE & IS \\
ITEM & IS \\
\hline
\end{tabular}

Fgure 6-1. A demarcation of the subsidiary company financing problem

This table shows for each object class whether it is viewed as part of the problem situation's IS or RS. Everything else is considered to be part of the IS/RS-combination's environment. Observe that BUDGET, ALTERNATIVE, and ITEM are viewed as part of the IS, even though they are entirely subject to manipulation by the participants. The reason behind this demarcation is that budget, alternatives, and items are abstract notions that figure only in the participants' decision process. They do not belong to the category of "concrete" objects like COMPANY and ARTICLE. If the recursion principle were applied to the IS demarcated as in Figure 6-1, resulting in the combination $\mathrm{IS}_{1} / \mathrm{RS}_{1}$, the participants would figure in $\mathrm{IS}_{1}$, and the budget, alternatives, and items in $\mathrm{RS}_{1}$.

This demarcation marks the end of our conceptualization activity. The resulting conceptual model consists of the object classes we defined, and our demarcation of the IS, the RS, and the environment. The class definitions should provide sufficient concepts to describe the problem-solving processes that take place within the IS. Figure 6-2 shows a graphical representation of the conceptual model. Note that a dashed arrow indicates that the corresponding action does not change the affected object directly, but rather induces the affected object to perform some action of its own.

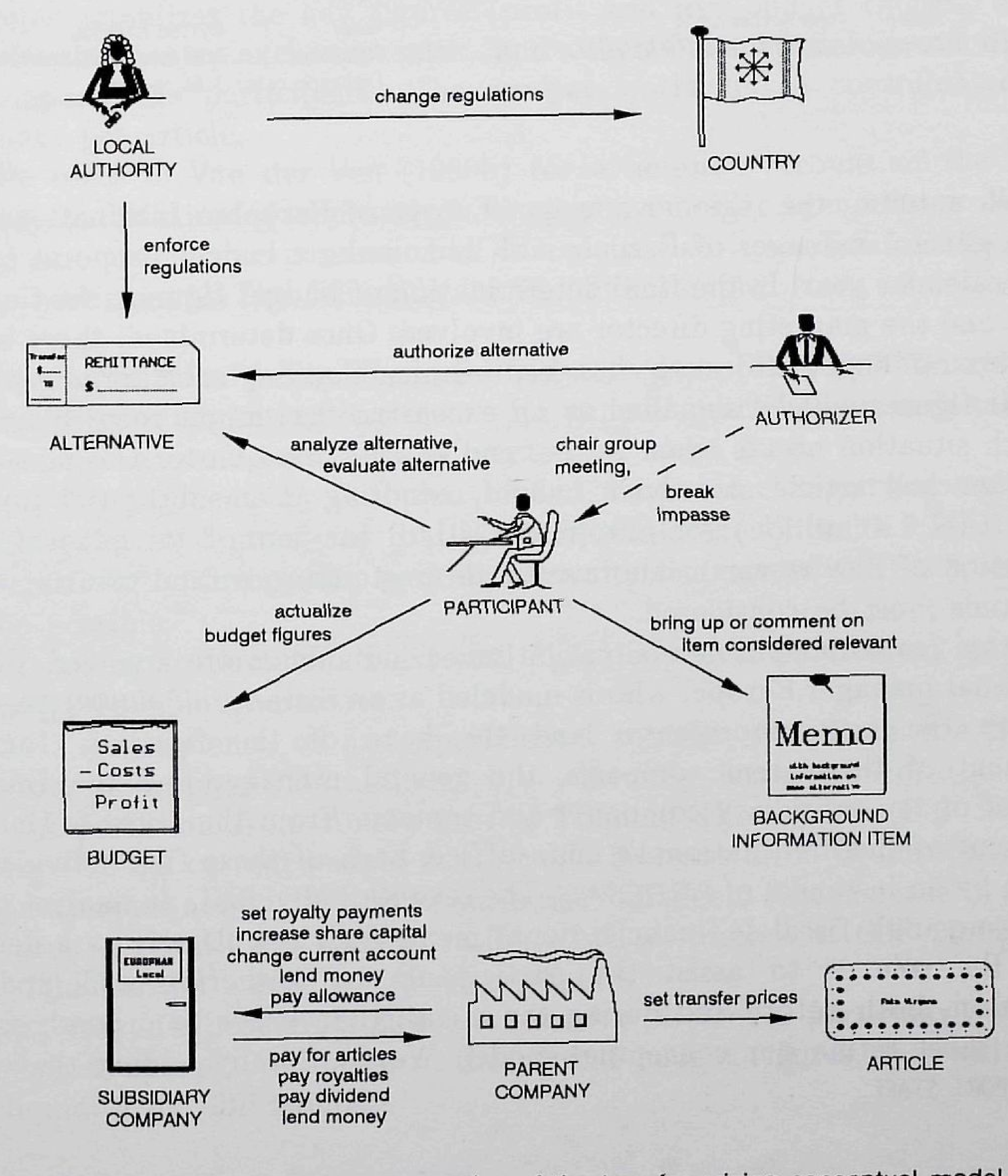




\subsection{SPECIFICATION}

A highly detailed empirical model of the transfer pricing problem situation is described by Van der Ven (1989a). In this section we shall only relate those aspects that are essential for a proper understanding of the task structures that will be presented in the next section.

The empirical model contains two companies belonging to the same multinational group:

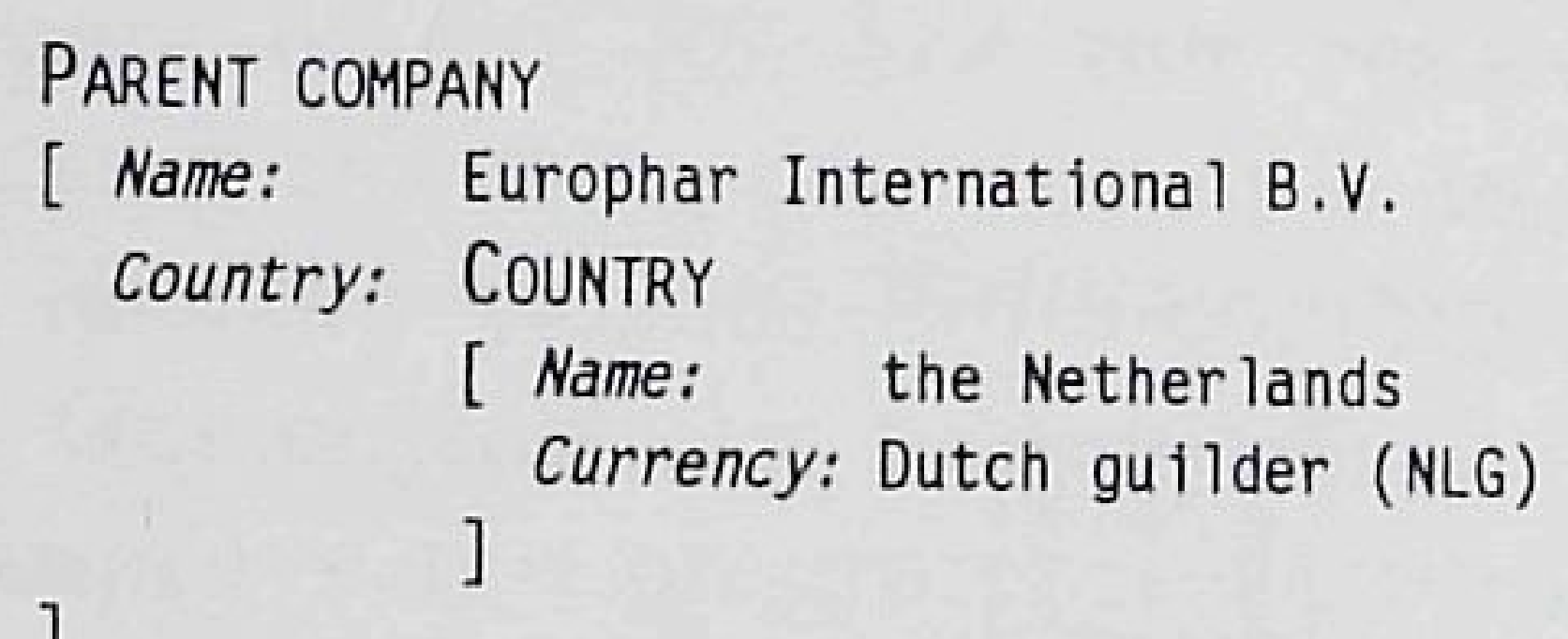

Each autumn, the regional manager Europe of Europhar International B.V. and the general manager of Europhar UK Ltd. make a budget proposal for the coming calendar year. In the final determination of budget figures, the financial director and the marketing director are involved. Once determined, these budget figures are no longer subject to changes, meaning that any substantial deviation in actual figures will be signalled as an exception that might require action.

Such situation occurs when by the end of the first quarter the sales for a newly launched article are above budget, resulting in a substantial liquidity surplus (GBP 1.4 million) for Europhar UK Ltd. In view of the plans for the construction of new research laboratories in Amsterdam, a fund transfer to the Netherlands must be considered.

In this particular problem situation seven individuals are involved. Firstly, the regional manager Europe, who is modeled as an instance of AUTHORIZER. Next, there are the price coordinator and the head of the fiscal \& financial department of the parent company, the general manager and the financial controller of the subsidiary company, and someone from the fiscal \& financial department of the multinational's main office. Each of these five individuals is modeled by an instances of PARTICIPANT. The seventh individual, an analyst of the parent company's fiscal \& financial department, does not classify as a decision maker. His role is to assist the participants by gathering and updating information, both before and during the meeting. In order to properly model him, we must refine our conceptual model. We do this by adding the object class SUPPORT STAFF.

\author{
object class SUPPORT STAFF \\ [ attributes \\ Company The specific company within the multinational for which this support \\ staff member works. \\ Function The function this support staff member holds within that company
}

actions

assist participant in actualizing figures

look up specific information on articles during group meeting

Since other, more urgent problems tie up some of the participants, a group meeting is scheduled for July. Meanwhile, the subsidiary company's financial controller actualizes the key figures (profit and loss budget figures, expected sales developments, exchange rates, and other financial data) and distribute these among the participants. The analyst works out a consolidated profit statement per article.

We refer to Van der Ven (1989b) for a detailed account of the meeting and the combination of alternatives that has eventually been chosen. However, we do wish to give an impression of the variety of items that are brought up by the participants. The following list is a fair sample:

- Instead of transferring all surplus to Amsterdam, GBP 240.000 could be used to set up a marketing conference for general practitioners in Britain. Plans for such a conference already existed for some time.

Royalty payments by Europhar UK Ltd. have not changed for eight years, not even to compensate for inflation, while the UK sales have increased significantly during that period. A royalty increase of GBP 670,000 should be possible.

- According to Eurochem's external fiscal consultants, dividend payment is not a feasible alternative.

As a consequence of an experimental contract for the entire Eurochem group, Europhar International B.V. currently bears part of the costs of transportation from Amsterdam to London. These costs (GBP 200,000 in view of the current budget sales) could be transferred to Europhar UK Ltd.

Some other articles also show sales above budget. This increase in sales will result in additional transportation costs, estimated at GBP 50,000. These could also be borne by Europhar UK Ltd.

- Recently, the minimum strategic transfer price of a particular product has been increased. According to the current budget figures, this will decrease liquidity by GBP 240,000 . 
- Some products currently are packaged in the United Kingdom with a gross margin of GBP 200,000. Relocation of the packaging activities to the Netherlands would reduce UK liquidity by an equal amount.

- The Dutch packing plant has capacity problems, meaning that the previous alternative is not feasible.

Observe that these items relate not only to financing alternatives (dividend, royalties, cost transfer, and transfer prices), but also to operational alternatives (the marketing congress) and relocation alternatives (the transfer of packaging activities).

Obviously, our empirical model of the transfer pricing problem differs significantly from that of the shiftwork planning problem in the previous chapter. There we could specify the exact relationship between the actions of a personnel manager and the individual rosters, while the tallies provided us with the means for correspondence checking. In the transfer pricing situation our means to determine the degree of correspondence of our empirical model are limited to comparing the attribute values of companies, articles, countries, and participants involved.

\subsection{TASK ANALYSIS}

In this section we present two task structures of the subsidiary company financing problem: The first one pictures the problem-solving process as observed prior to any strucuring, the other constitutes a programme that specifies how the problem can be solved in a more structured way. Figure 6-3 shows the global structure of the original subsidiary company financing task. All participants continuously scan the environment for situations that might require a group meeting. During such a meeting, decisions are made with respect to financing, relocation, as well as operational alternatives.

Usually, some combination of alternatives is required to arrive at a complete solution. Thus, alternatives may constitute a partial solution in the sense that they effectuate a transfer of only part of the total amount required. When a partial solution is found, and agreed upon by all participants, it must be authorized before it can be implemented. Depending on the authorization regulations that exist within the company, either the authorizer's formal agreement suffices, or higher level authorization is required.

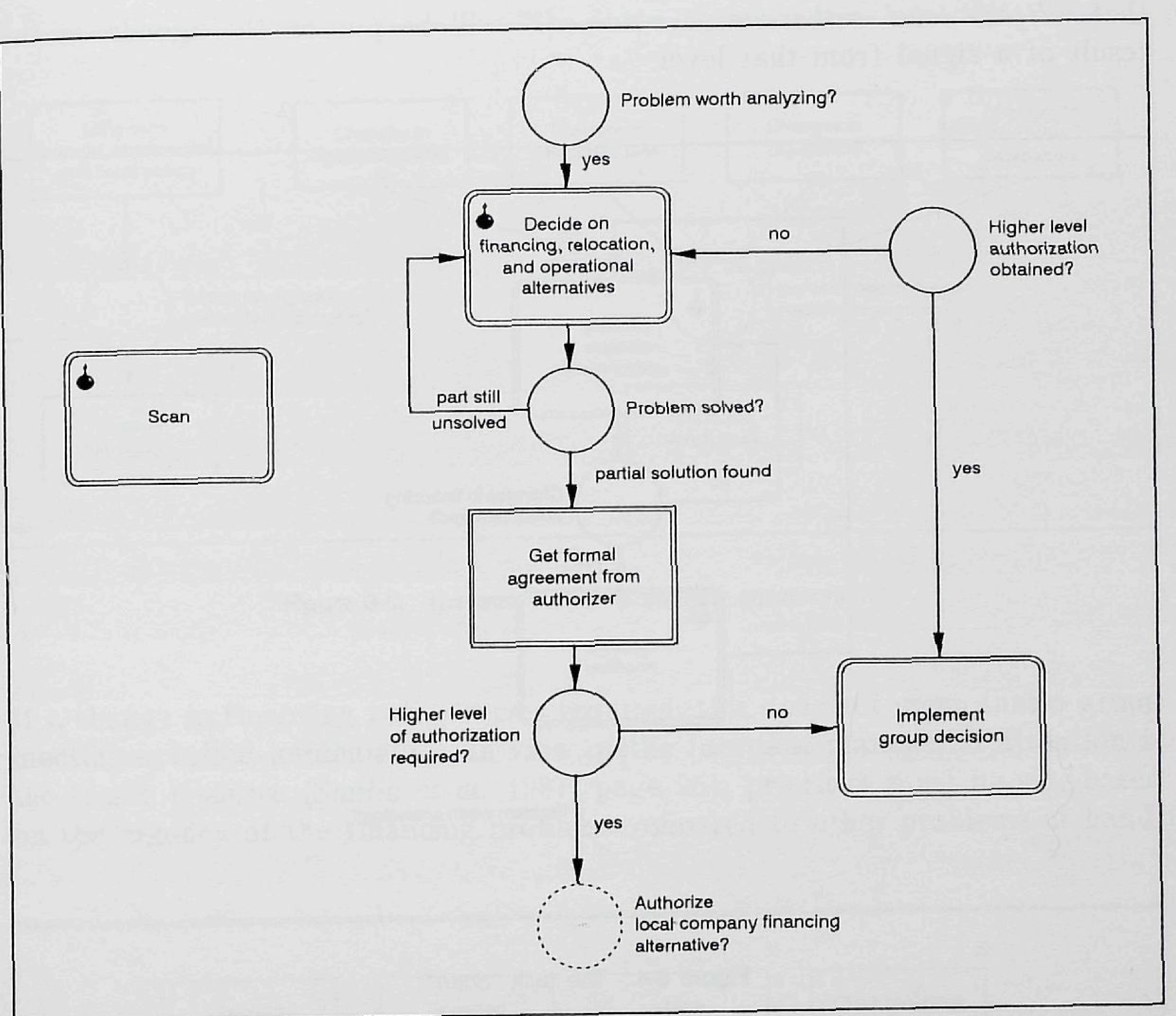

Flgure 6-3. The task "Finance subsidiary company"

Note that, according to the definition on page 46 , the subtask "Scan" is the only initial item of "Finance subsidiary company". Although invisible in this flow diagram, the decisions "Problem worth analyzing?" and "Higher level authorization obtained?" have incoming edges. As can be seen in Figure 6-4, the authorization obtained?" have incoming edges. As can be seen in "Scan". Similarly, "Higher level authorization obtained?" is signalled from some task at a higher managemen level. Since this task is not performed by any participant in our empirical level. Since this task is not performed by any participant in the transfer pricing problem. We therefore use signals to model the coordination between the two management levels involved. It suffices to know that the decision "Authorize subsidiary company financing alternative?" is made on some higher level, and 
that "Higher level authorization obtained?" will be put on the agenda as the result of a signal from that level.

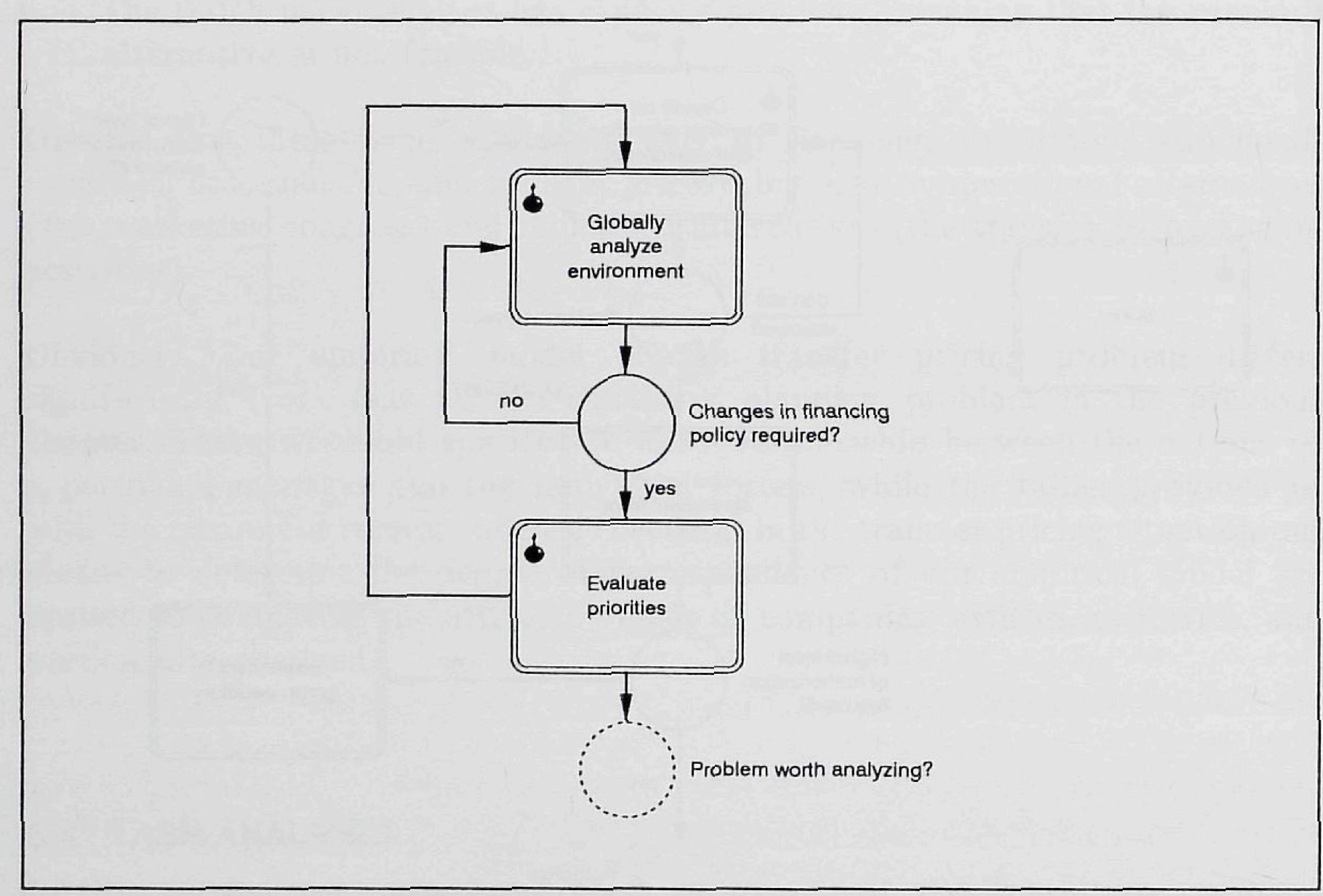

Figure 64. The task "Scan"

Two subtasks are identified in the scanning task: "Globally analyze environment" and "Evaluate priorities". The former comes down to determining the impact of changes in the various areas on the company's key figures (the profit and loss account, liquidity, etcetera), and comparing these with the key figures that are desirable in view of the long-term financial, fiscal, and commercial policy. The latter means determining whether the difference between these two sets of figures is of such nature that calling a group meeting is justified. The decision structure of these two subtasks is shown in Figure 6-5 and 6-6.

The global analysis of the environment is performed constantly by all participants. Their information sources include reports containing budget and other key figures from the company's management information system, but all kinds of informal communication - "hearsay" - as well.

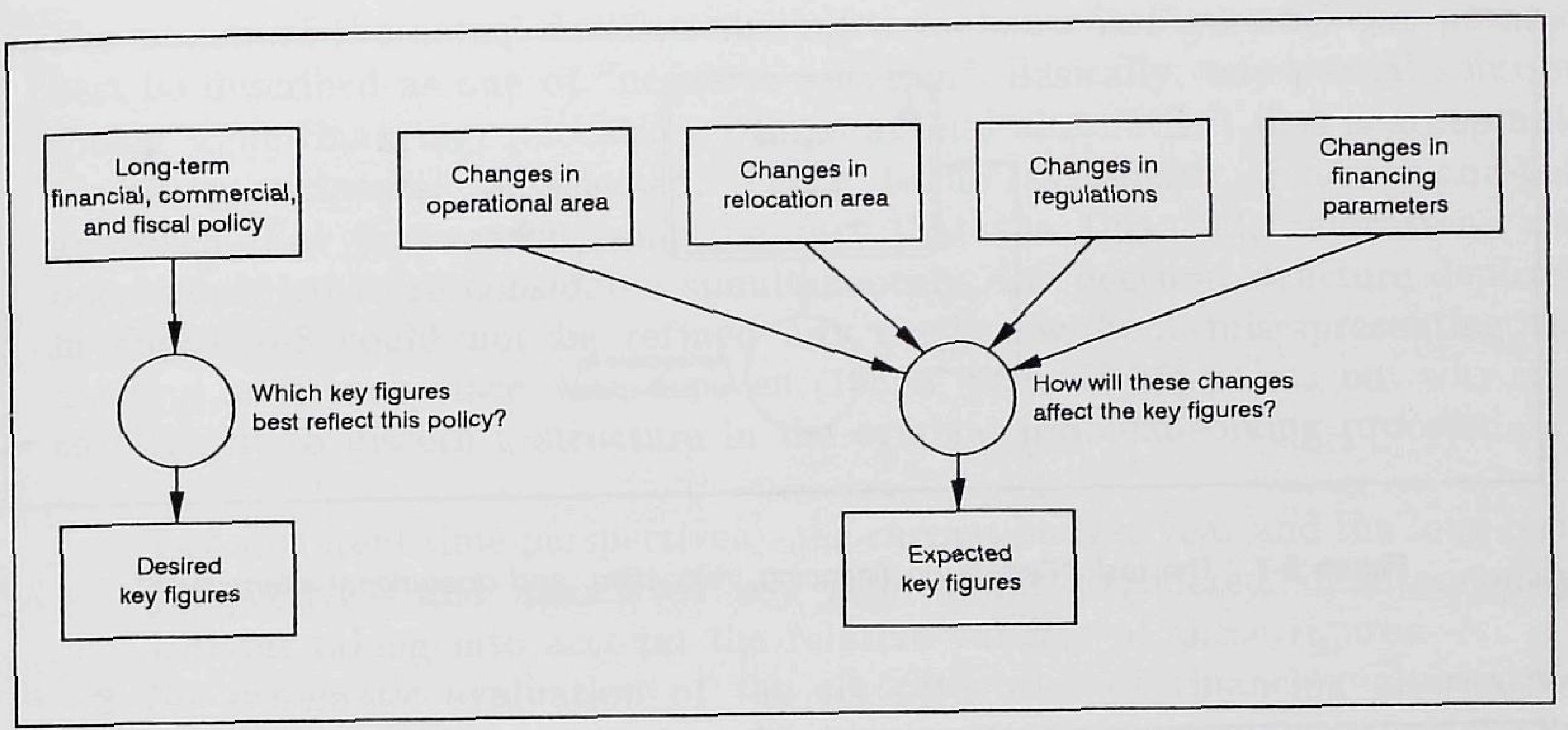

Figure 6-5. The task "Globally analyze environment"

If a change in financing policy seems required, this does not mean that a group meeting is called immediately. In view of the fact that managerial attention is the scarce resource (Simon et al. 1987, page 25), priorities must be set, based on the urgency of the financing problem compared to other problems at hand.

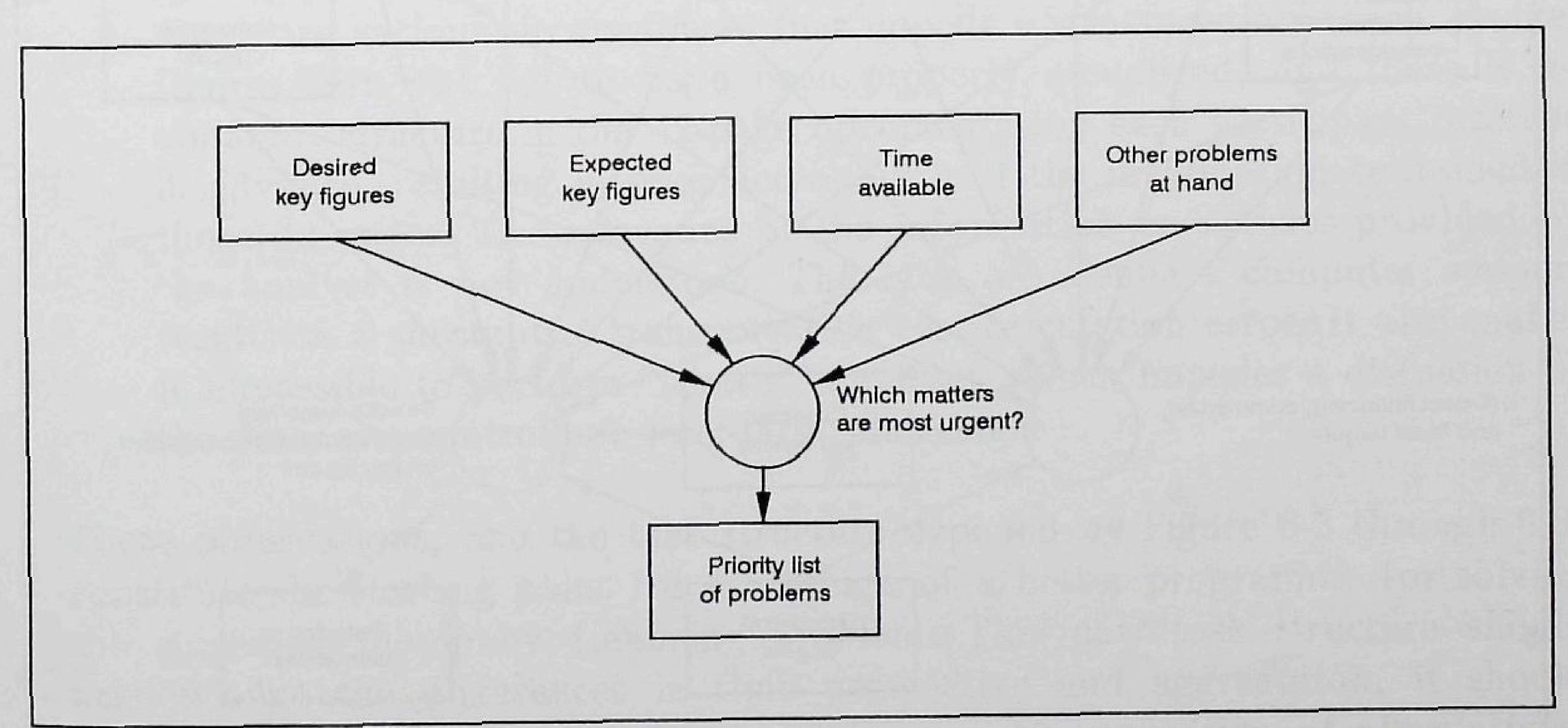

Figure 66. The task "Evaluate priorities" 


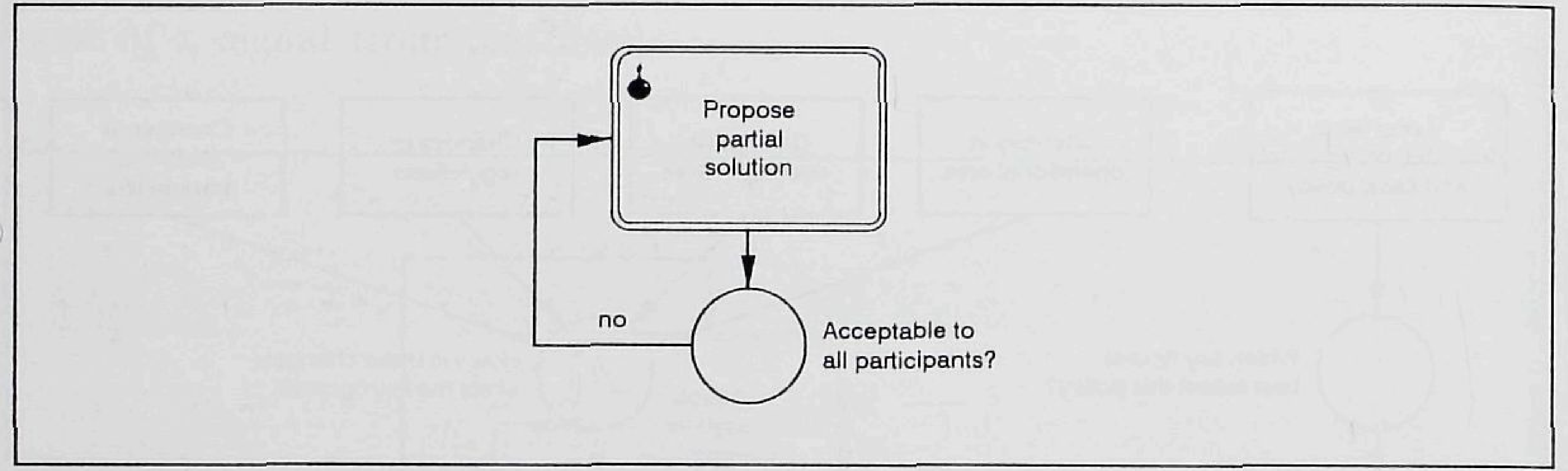

Flgure 6-7. The task "Decide on financing, relocation, and operational alternatives"

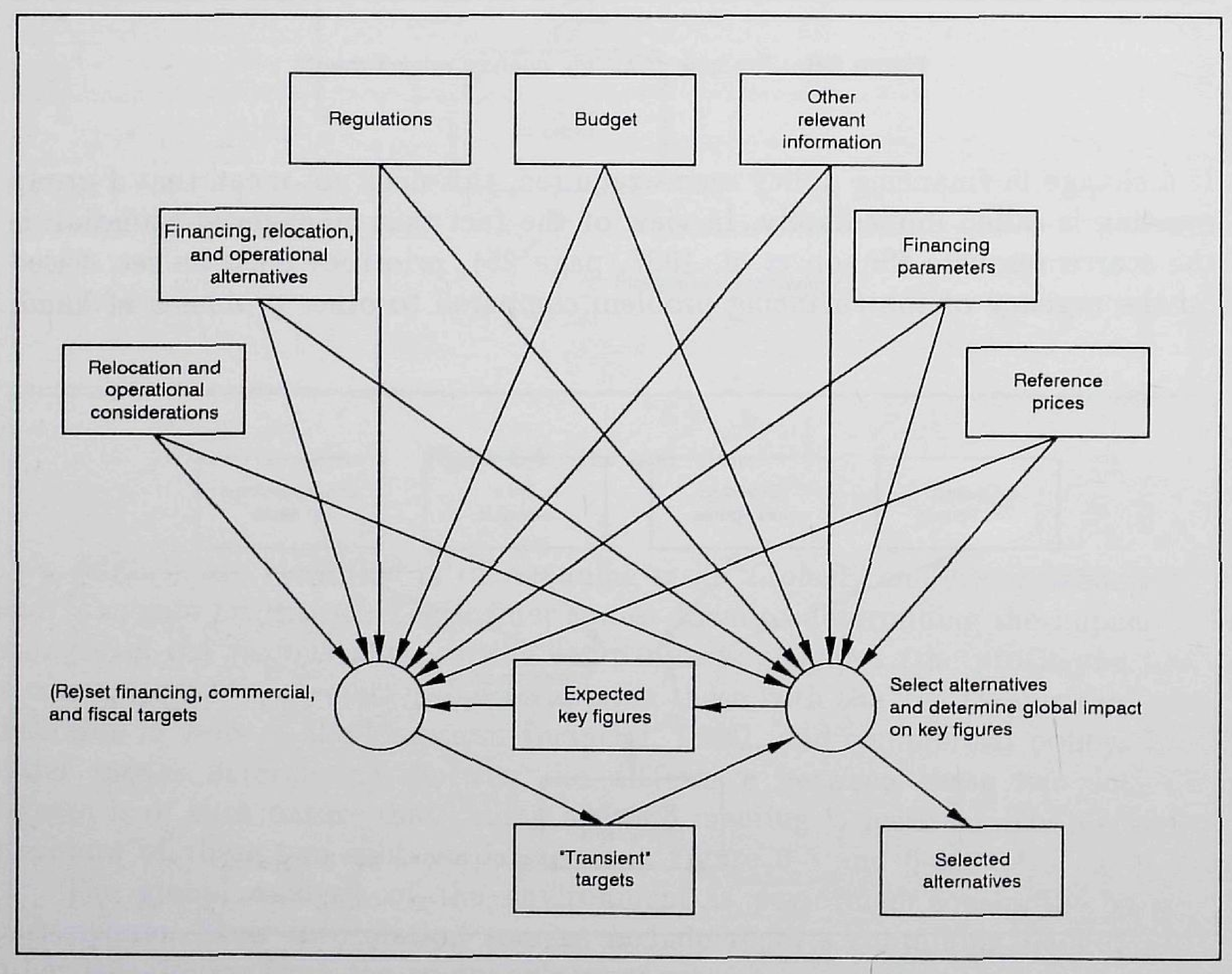

Fgure 6-8. The task "Propose partial solution"
The nature of the actual decision-making task shown in Figure 6-7 can perhaps best be described as one of "negative selection". Basically, any partial solution (being some financing, relocation, or operational alternative) that is acceptable to all participants, is selected. There is no systematic generate-and-test approach. For this reason, and the fact that the financial, relocation, and operational area are considered simultaneously, the decision structure depicted in Figure 6-8 could not be refined any further without misrepresenting the meeting as it took place. Van der Ven (1989a, page 78-81) points out why it is so difficult to discern a structure in the original problem-solving process:

1. Two different time perspectives - the current budget year and the long term perspective - and associated key figures are considered simultaneously, without taking into account the relative validity of these figures.

2. No systematic evaluation of the six categories of financing alternatives takes place. When reviewing the group meeting, no explanation can be found for the fact that local lending, a change in the current account, or an intra-company loan have not been considered.

3. When the transfer price alternative is considered, no clear distinction between the aggregate and disaggregate level is made. The total amount to be transferred (aggregate level) is translated immediately into a transfer price increase for one specific article, and later another (disaggregate level). The decision to use these two articles in particular is made without any systematic search at the disaggregate level.

4. There are various circumstances that impede a structured approach. Budget figures turn out not to have been properly actualized, and there is no apparent structure in the 42-page documentation each participant received in advance, resulting in poor accessibility of the information contained in those 42 pages. The relevance of the information on articles provided by the analyst is not understood. The lack of adequate computer support results in a substantial transportation cost calculation error. It also makes it impossible to perform "what-if" analyses, which impedes a discussion of the financial controller's long-term planning.

These observations, and the task structure depicted by Figure 6-3 through 6-8, constitute the starting point for the design of a better programme for solving the subsidiary company financing problem. This new task structure should acknowledge the differences in time perspective and aggregation, it should incorporate preparatory activities and a systematic evaluation of alternatives, and its leaf tasks should clearly define the decisions that have to be made and the information that is to be used/generated when making these decisions. In the remainder of this section we present a task structure that meets these demands to a large extent. 
Recall that at the close of Section 6.2 we restricted our conceptual model to include financing alternatives only. However, this boundary is not respected in Figure 6-3. The task "Decide on financing, relocation, and operational alternatives" has therefore been replaced by "Decide on financing alternatives only" in Figure 6-9. The purpose of this task is to find the best combination of fund transfer alternatives in the financing area. This means that it is only put on the agenda a second time when this combination of alternatives cannot be authorized and a new combination must be looked for. Otherwise, a solution must be looked for in the operational or relocation area, since all possibilities in the financing area have been considered. This coordinating mechanism is modeled as a signal: The decision to search for operational or relocation alternatives is beyond the scope of the financing task.

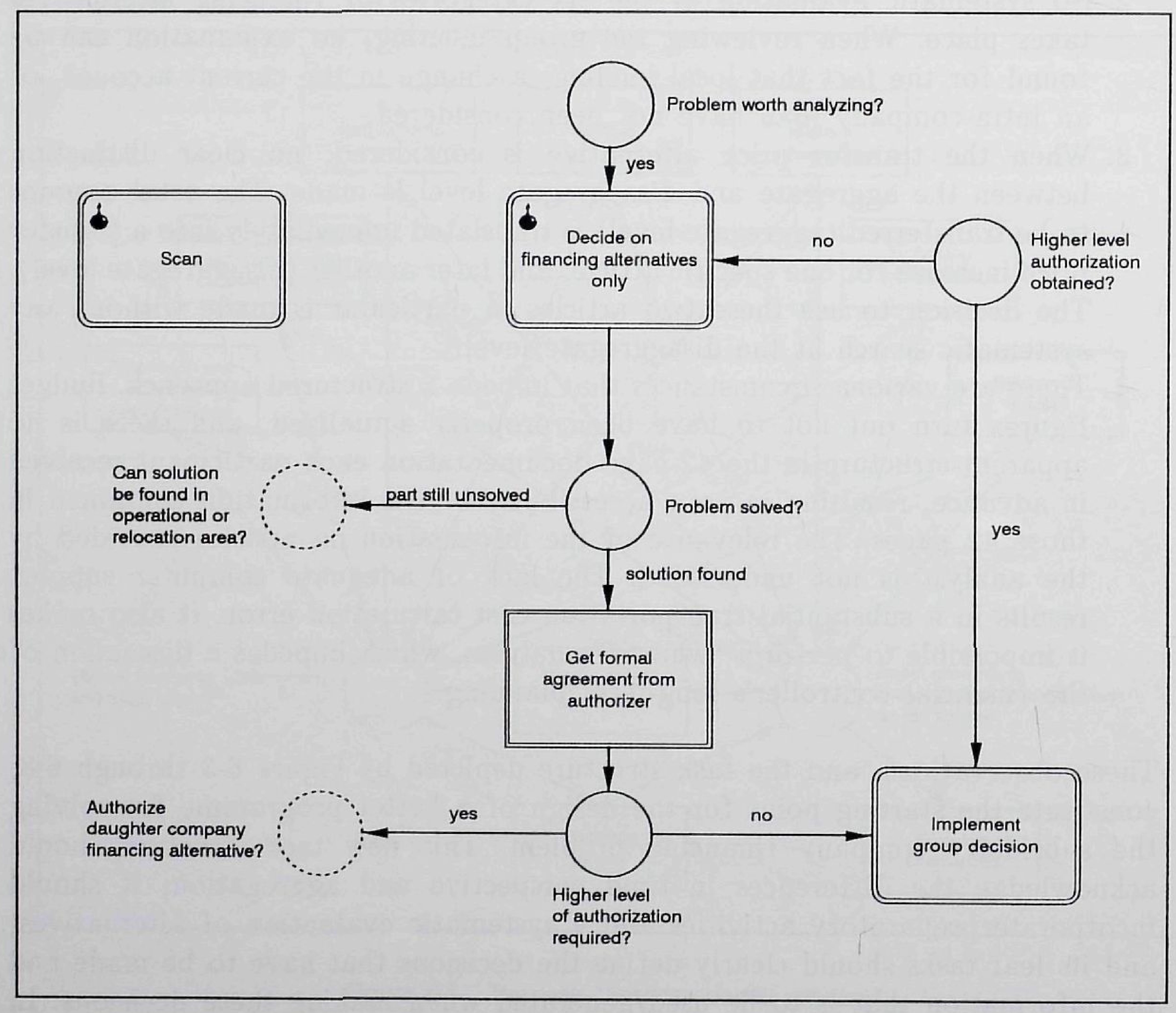

Fgure 6-9. The revised structure of task "Finance subsidiary company"
The structure of the task "Decide on financing alternatives only" is essentially a "blow-up" of Figure 6-7, to which the preparatory task has been added. This preparatory task should eliminate the first two problems mentioned under point 4 on page 151.

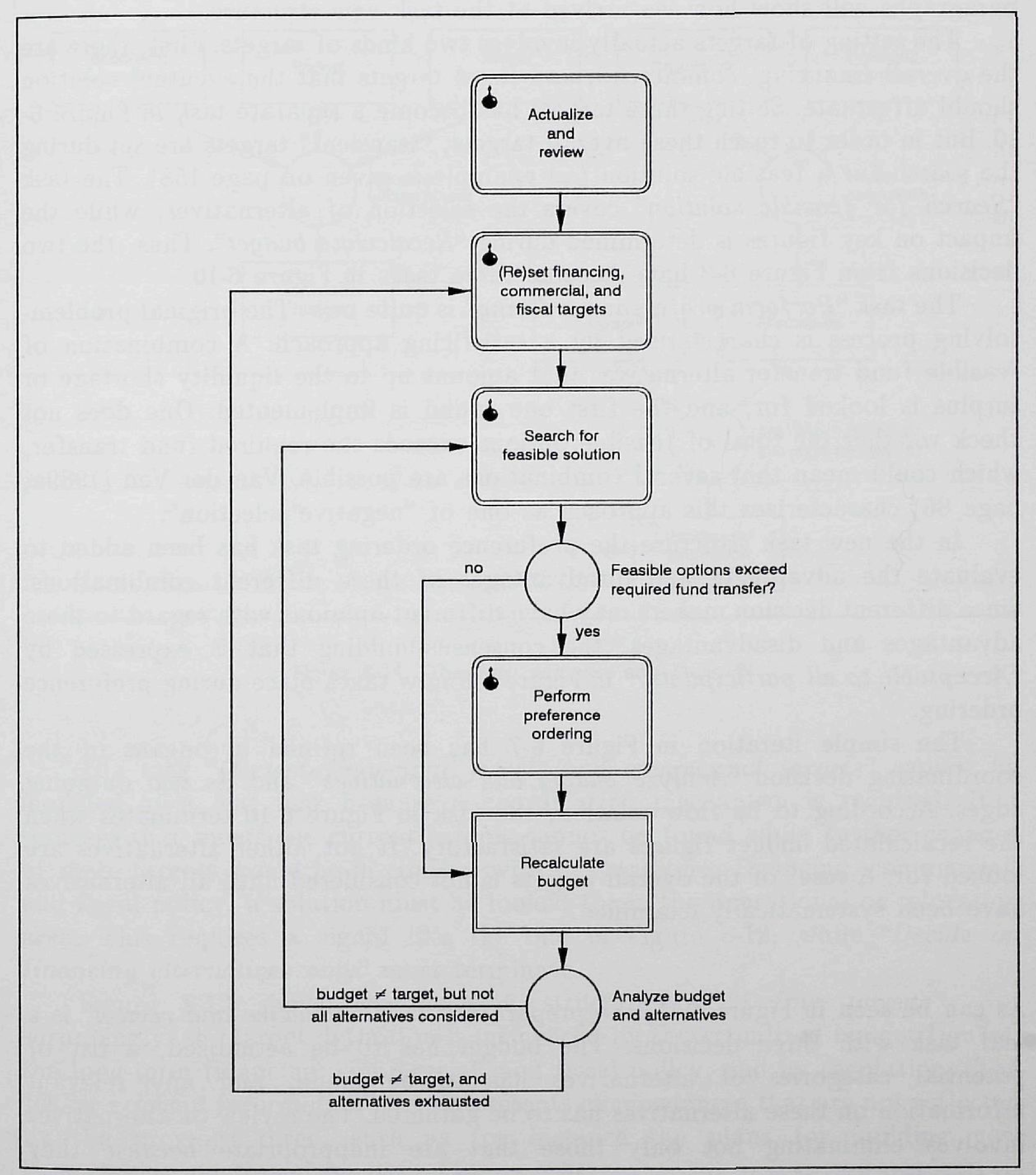

Fgure 6-10. The task "Decide on financing alternatives only" 
The main loop "repeat until solution has been found" of Figure 6-7 has of course been refined. This refinement is based partly on the two decisions of the task "Propose partial solution" depicted by Figure 6-8 ("Reset financing, commercial, and fiscal targets" and "Select alternatives and determine global impact on key figures"), partly on other considerations. The following paragraphs will show how we arrived at the task new structure.

The setting of targets actually involves two kinds of targets. First, there are the overall financing, commercial, and fiscal targets that the eventual solution should effectuate. Setting these targets has become a separate task in Figure 610. But in order to reach these overall targets, "transient" targets are set during the search for a feasible solution (an example is given on page 158). The task "Search for feasible solution" covers the selection of alternatives, while the impact on key figures is determined during "Recalculate budget". Thus, the two decisions from Figure 6-8 have become three tasks in Figure 6-10.

The task "Perform preference ordering" is quite new. The original problemsolving process is characterized by a satisficing approach: A combination of feasible fund transfer alternatives that amount up to the liquidity shortage or surplus is looked for, and the first one found is implemented. One does not check whether the total of feasible options exceeds the required fund transfer, which could mean that several combinations are possible. Van der Ven (1989a, page 86) characterizes this approach as one of "negative selection".

In the new task structure the preference ordering task has been added to evaluate the advantages and disadvantages of these different combinations. Since different decision makers may have different opinions with regard to these advantages and disadvantages, the consensus-building that is expressed by "Acceptable to all participants?" in Figure 6-8 now takes place during preference ordering.

The simple iteration in Figure 6-7 has been refined by means of the coordinating decision "Analyze budget and alternatives" and its two outgoing edges. According to its flow relation, the task in Figure 6-10 terminates when the recalculated budget figures are satisfactory. If not, other alternatives are looked for. A reset of the overall targets is not considered until all alternatives have been systematically examined.

As can be seen in Figure 6-11, the preparatory task "Actualize and review" is a leaf task with three decisions: The budget has to be actualized, a list of potential categories of alternatives has to be made, and any relevant information on these alternatives has to be gathered. The review of alternatives involves eliminating not only those that are inappropriate because they effectuate a transfer in the wrong direction (royalty increase is not appropriate in case of a liquidity shortage), but also those that are not feasible in view of regulations or financing parameters (recall the dividend issue mentioned on page 145).

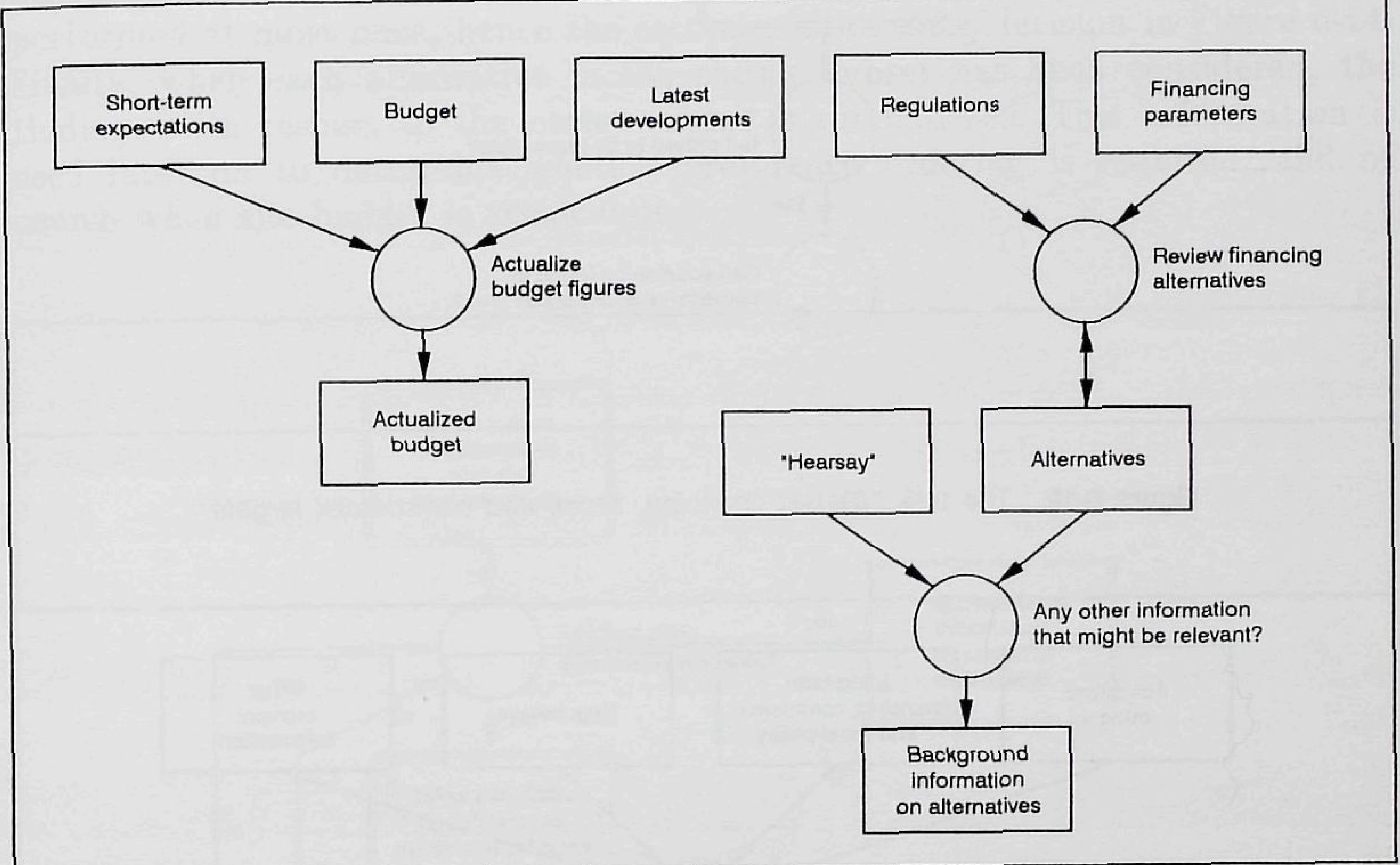

Fgure 6-11. The task "Actualize and Review"

The task "(Re)set financing, fiscal, and commercial targets" cannot be modeled as a leaf task because a coordinating mechanism is required: If a solution that meets the current targets cannot be found while further changes of these targets would be in conflict with the long-term financing, commercial, and fiscal policy, a solution must be looked for in the operational or relocation area. This requires a signal like the one in Figure 6-12, while "Decide on financing alternatives only" must terminate.

Figure 6-13 shows the decision structure of "Define targets". No surprisingly, the target definition is influenced by the actualized budget figures, the long-term financing, commercial, and fiscal policy, and by regulations. The "Other relevant information" item represents circumstances that are not reflected by the previous three, such as for instance the plans for building new laboratories mentioned on page 144 . 


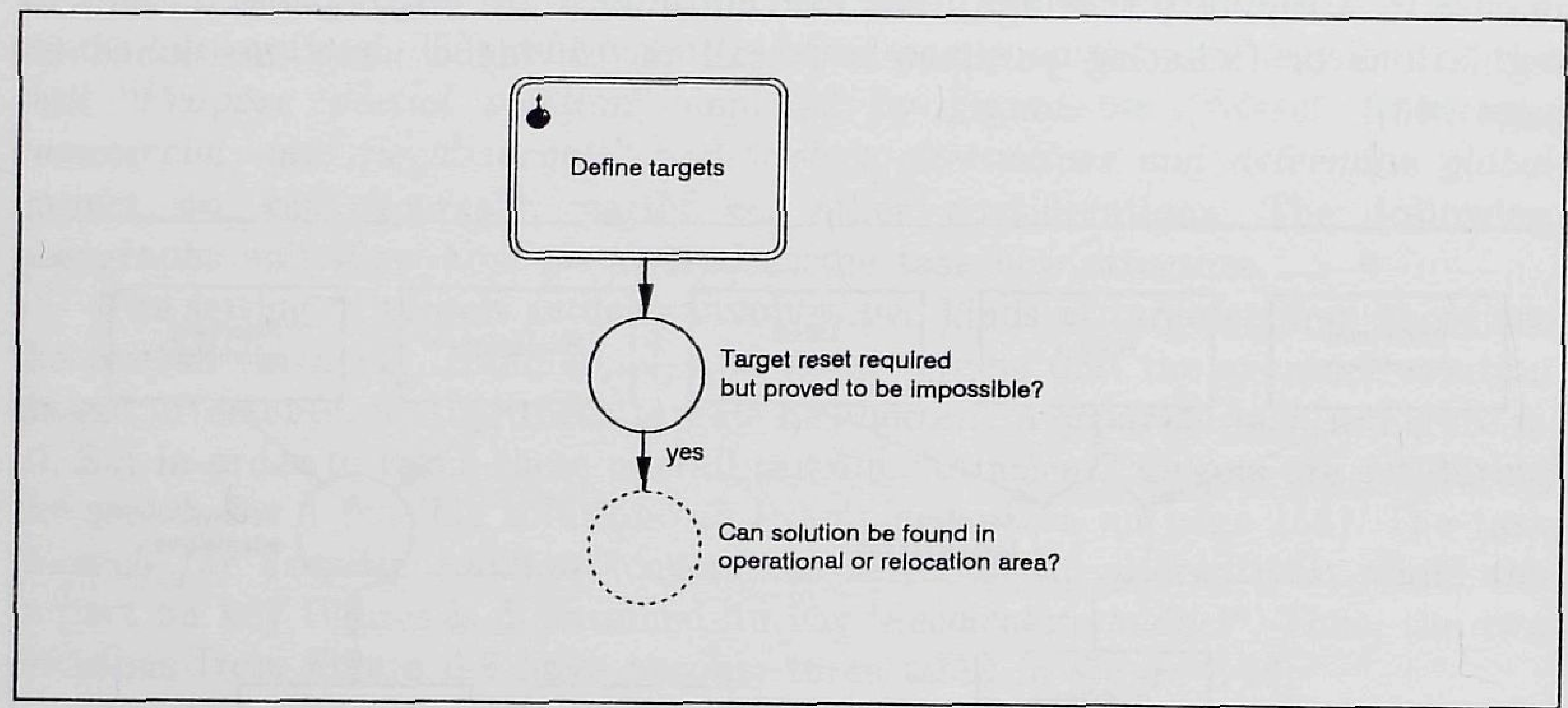

Flgure 6-12. The task "(Re)set financing, fiscal, and commercial targets"

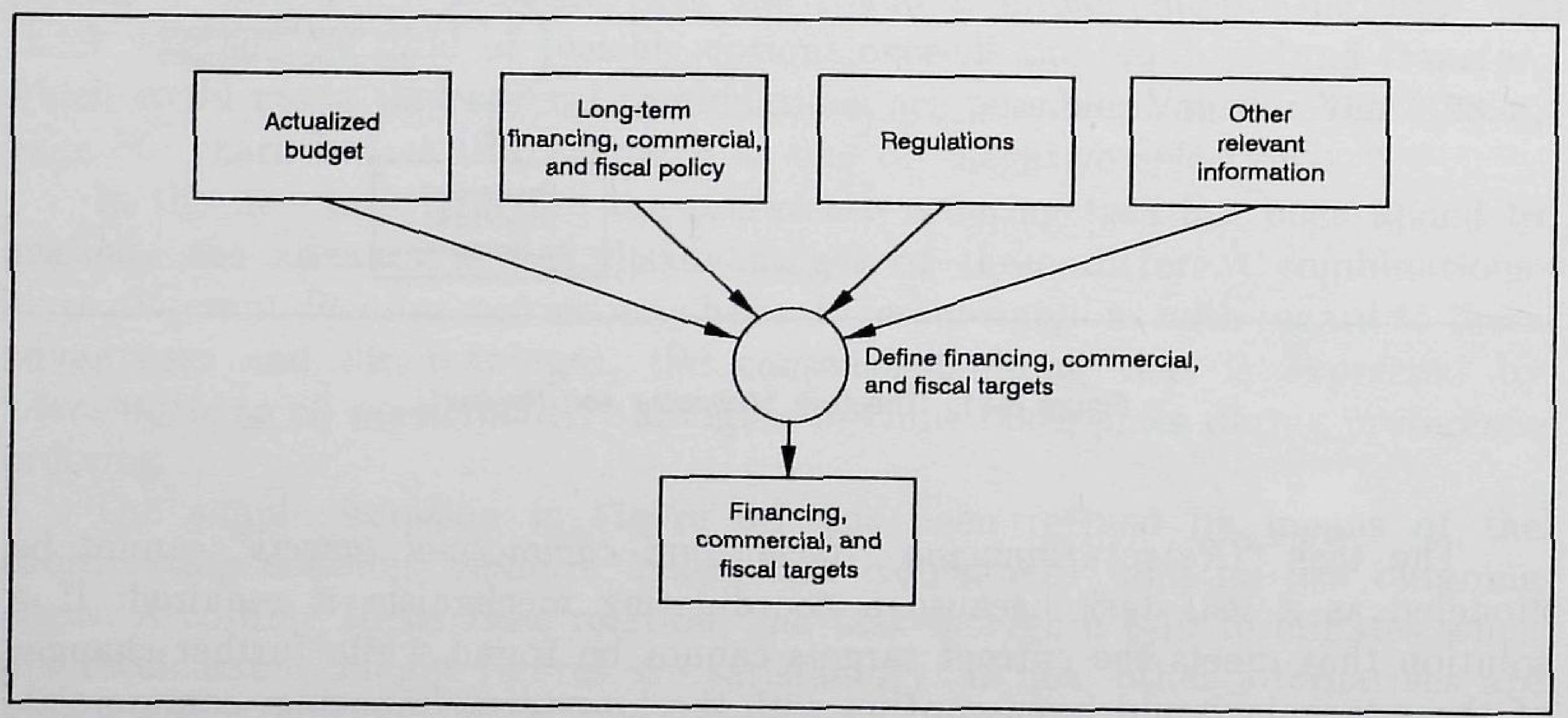

Figure 6-13. The task "Define targets"

Observe that by structuring the task "Decide on financing alternatives only" as is shown in Figure 6-10, we largely eliminate the problem mentioned under point 2 on page 151: Targets are not reset until all alternatives have been considered. Obviously, much still depends on the structure of the feasible solution search. Within this task we can identify four subtasks, as is shown in Figure 6-14. The search starts with selecting a subset of alternatives and setting the total remittance that should be accomplished by means of that subset. Subsequently, each alternative in this subset is considered in order to determine the maximal amount of funds that can be transferred by means of that alternative. If this alternative involves a change in transfer prices, it must be considered at disaggregate level as well, since the maximal remittance also depends on the attribute values of specific articles. This analysis at disaggregate level has to be performed at most once, hence the second coordinating decision in Figure 6-14. Finally, when each alternative in the chosen subset has been considered, the findings with respect to the entire subset is summarized. This information is used later on to determine whether preference ordering is required, and of course when the budget is recalculated.

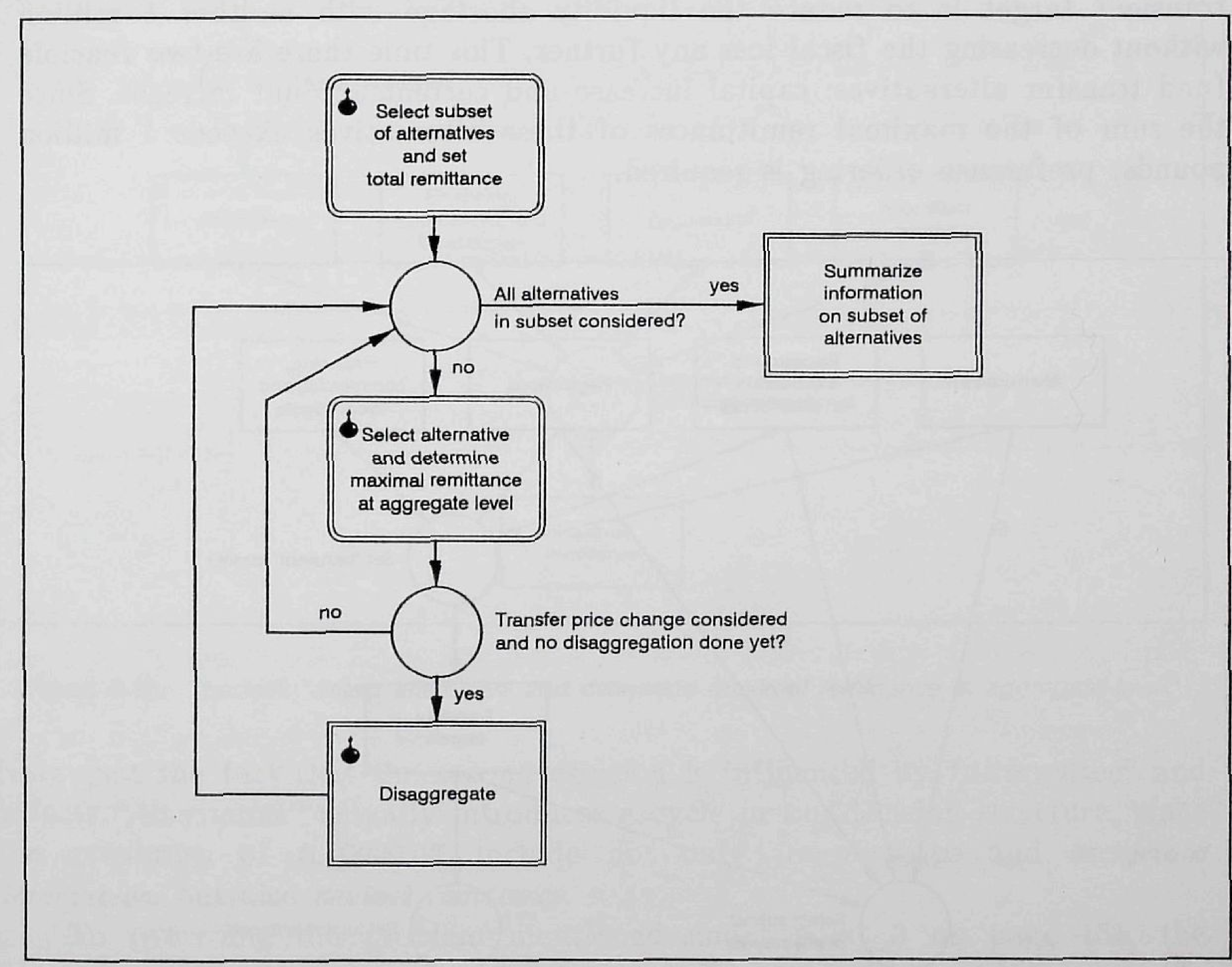

Floure 6-14. The task "Search for feasible solution"

Figure 6-15 depicts the decision structure of "Select subset of alternatives and set total remittance". The choice of alternatives depends on the specific nature of the subsidiary company financing problem. For example, Van der Ven (1989a, page 122-125), describes a problem situation in which the actualized budget 
figures indicate for Europhar UK Ltd. a liquidity shortage of 2.5 million, and a fiscal loss of 2.4 million. The financial, commercial, and fiscal targets consist of a small profit, neither liquidity shortage nor liquidity surplus, and a fisca loss of 0.9 million. This means that 1.5 million of the liquidity shortage can be compensated using financing instruments that directly affect the profit and loss statement of the subsidiary company, while the remaining 1 million can be eliminated by means of alternatives that affect the balance sheet are. Thus, the first iteration has as "transient" targets to reduce both the liquidity shortage and the fiscal loss by 1.5 million. For this purpose only the transfer price decrease alternative is considered, since the other alternatives prove to be unfeasible. The second time "Select subset of alternatives and set total remittance" is active, the transient target is to reduce the liquidity shortage with another 1 million without decreasing the ficl lhe liquidity shortage with another 1 million fund fiscal loss any further. This time there are two feasible fund transfer alternatives: capital increase and current account increase. Since the sum of the maximal remittances of these alternatives exceeds 1 million pounds, preference ordering is required.

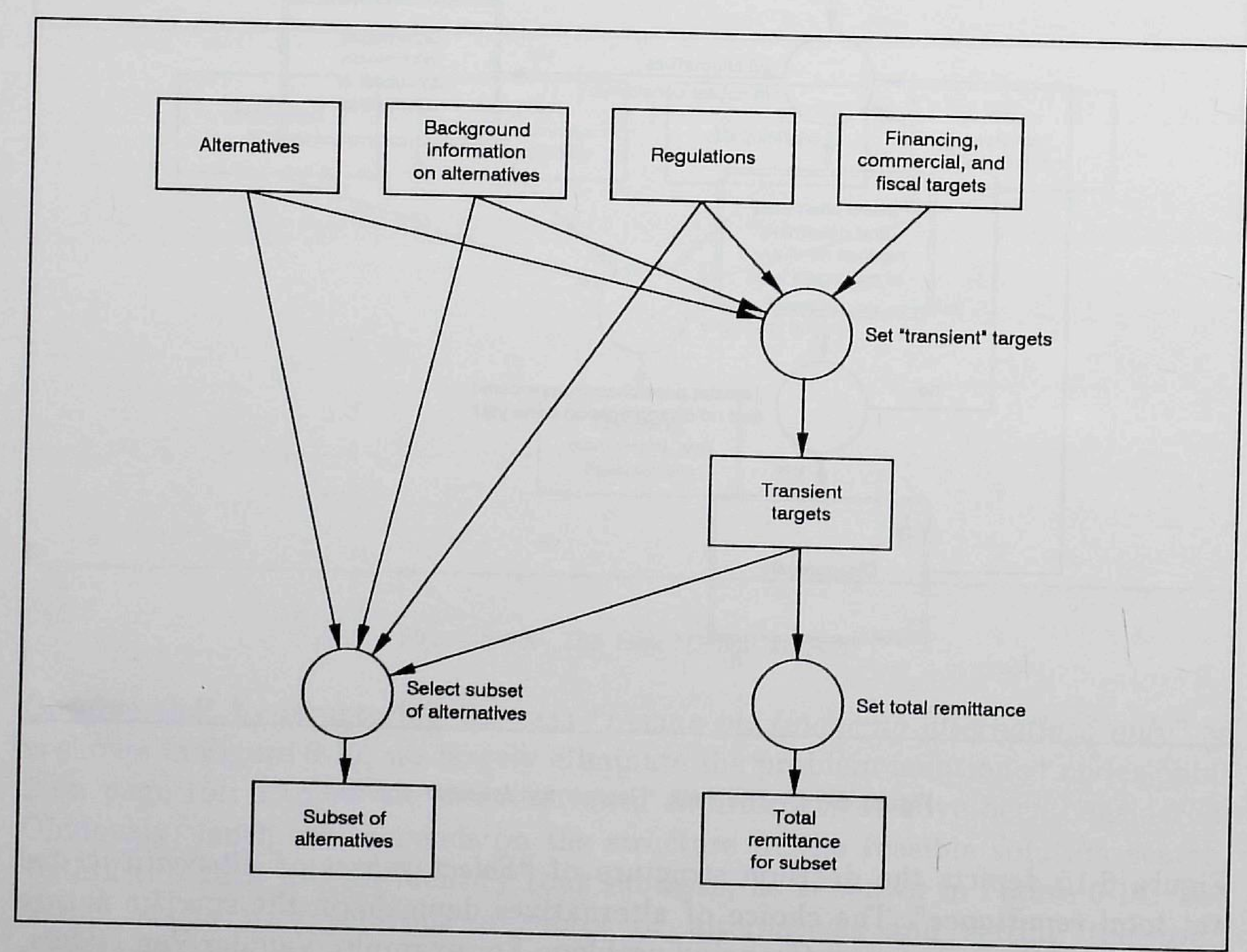

Fgure 6-15. The task "Select subset of alternatives and set total remittance"
The decision structure of "Select alternative and determine maximal remittance at aggregate level" is shown in Figure 6-16. Each alternative in the subset is selected once, and the participants decide on its maximal remittance, based on regulations, financing, commercial, and fiscal targets, and the actualized budget figures. If transfer prices are involved, the gross margin range that is acceptable also influences this decision.

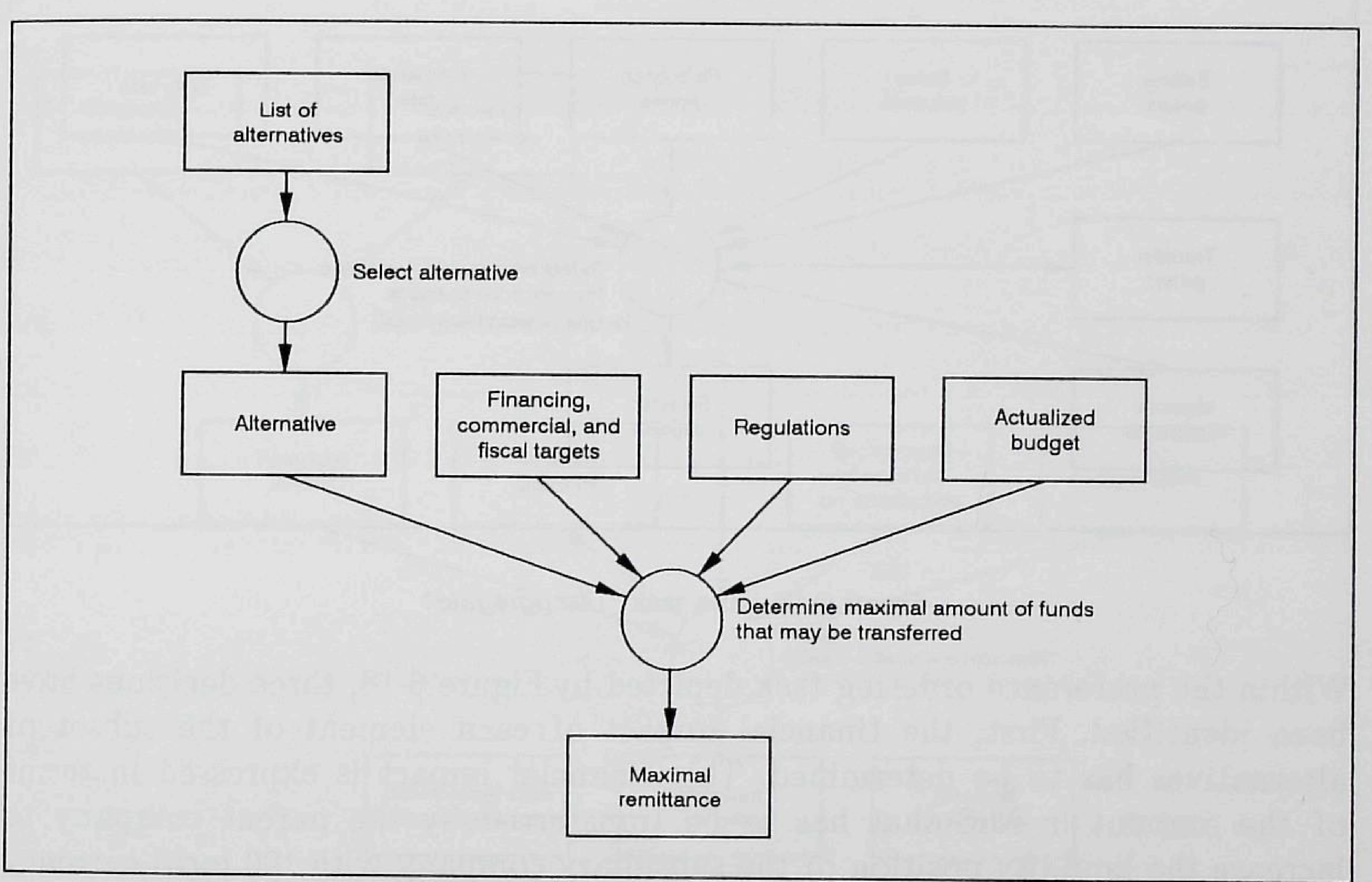

Fgure 6-16. The task "Select alternative and determine maximal remittance at aggregate level"

Note that the fact that the second decision is influenced by "Alternative" and affects "Alternative" actually introduces a cycle in the decision structure, since the attributes of ALTERNATIVE include not only Transfer type and Background information, but also Maximal remittance.

To overcome the problem mentioned under point 3 on page 151, the discussion of the transfer price alternative on aggregate level and disaggregate level are strictly separated. Figure 6-17 shows the decision structure of "Disaggregate". Basically, this task consists selecting articles that are suitable for a transfer price change, and determining their new prices. This has been modeled as a single decision. This decision is influenced by most attributes of ARTICLE, the maximal remittance that has been set for the transfer price alternative, and the current exchange rate and duty rate. It will affect "Selected 
articles", and of course the Transfer price attribute of these articles. It may turn out that only a few articles are suitable, or that the possible changes are only marginal, in which case the total remittance that seemed possible at aggregate level cannot be reached. For this reason, the decision "Select articles and determine transfer price changes" may also affect the maximal remittance.

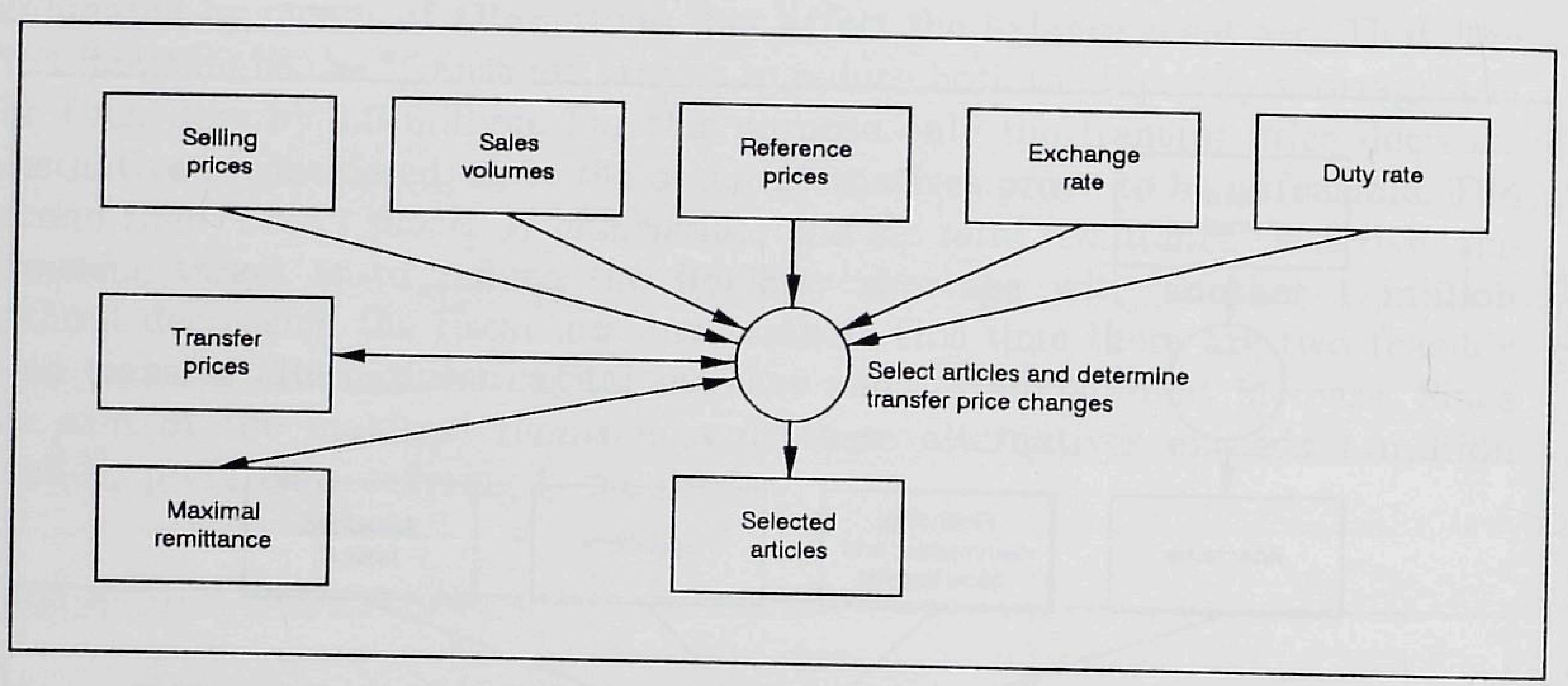

Fgure 6-17. The task "Disaggregate"

Within the preference ordering task depicted by Figure 6-18, three decisions have been identified. First, the financial impact of each element of the subset of alternatives has to be determined. This financial impact is expressed in term of the amount in NLG that has to be transferred by the parent company to increase the liquidity position of the subsidiary company with 100 local currency units. It mainly depends on financing parameters, such as exchange rate and duty rate (see page 166 for an example).

It would seem obvious that the alternative with the highest (or in case of a liquidity shortage the least negative) financial impact is to be preferred. But there are other important considerations as well. The next top therefore, is determine what these additional criteria are. This decision is influenced by regulations and the items of backgound information brought up during the meeting. Criteria that have been relevant in previous situations may again be of importance, so a checklist is maintained. In addition to financial impact, this list will typically contain considerations like flexibility and long-term effects. If some alternatives require a higher authorization level, this may also affect If some alternatives require a higher authorization level, this may also affect
the individual preferences.

Once the relevant criteria are agreed upon by all participants, they are used in determining the actual remittance (for each alternative in the subset the amount that should actually be transferred). Since most criteria are "soft" in comparison with the financial impact, each participant expresses his personal preferences, based on experience and "gut feeling". If opinions differ, consensus can be reached in a discussion that, in view of its highly specific topic and expertise-sharing nature, usually will be brief. Thus, the preference ordering task eventually results in a list containing for each fund transfer alternative in the subset the amount that is to be transferred by means of this alternative.

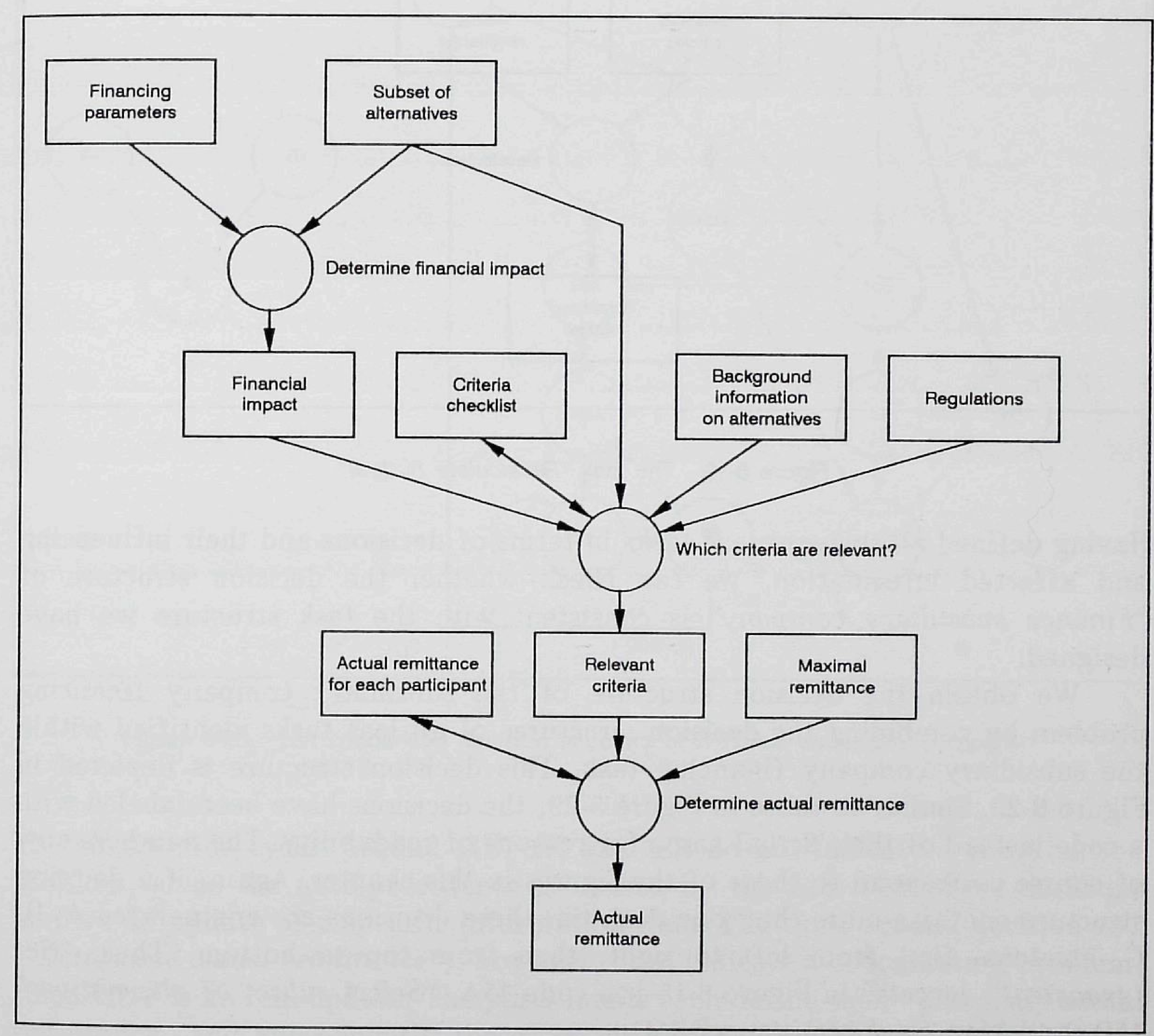

Fgure 6-18. The task "Perform preference ordering"

Figure 6-19 depicts the decision structure of "Recalculate budget" - the last leaf task of "Decide on financing alternatives only". This is a structured task, consisting of a single decision that can be fully automated. This decision is influenced by the actualized budget figures and the subset of financing 
alternatives that has been chosen (recall that the actual remittance corresponds to the Amount attribute of object class ALTERNATIVE). Recalculation results in a newly actualized budget, that is to say, a new short-term forecast that takes into account the effects of the agreed-upon fund transfers.

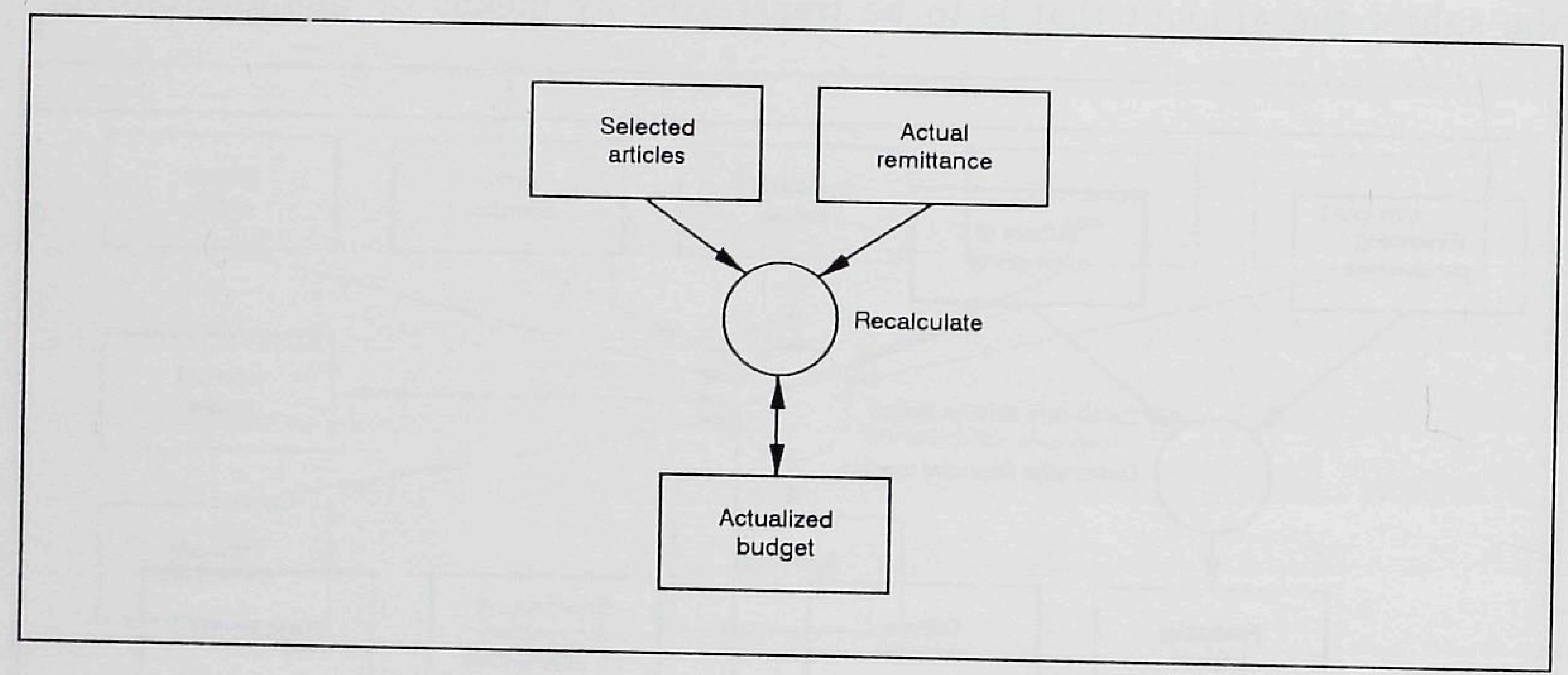

Fgure 6-19. The task "Recalculate budget"

Having defined all relevant leaf tasks in terms of decisions and their influencing and affected information, we can check whether the decision structure of "Finance subsidiary company" is consistent with the task structure we have designed.

We obtain the decision structure of the subsidiary company financing problem by combining the decision structures of all leaf tasks identified within the subsidiary company financing task. This decision structure is depicted the subsidiary company financing task. This decision structure is depicted in Figure 6-20. Similar to those in Figure 5-29, the decisions have been labeled with a code instead of their actual name for reasons of readability. The numbers now of course correspond to those of the figures in this chapter. Again, if a decision structure contains more than one decision, these C, etce decisions are enumerated A, B transient, first from left to right, then from top to bottom. Thus, "Set transient targets" in Figure 6-15 has code 15A, "Select subset of alternatives" 15B, and "Set total remittance" $15 \mathrm{C}$.

A study of the graph properties of the condensed decision structure in Figure 6-20 reveals no "real" inconsistencies in the sense that each of its cycles involving more than one decision can be found as a cycle in the task structure of "Finance subsidiary company". The cycle 16B-17-19 is covered by the inner loop in Figure 6-12, the larger cycle (a result of the edge 19-13) by the oute loop.

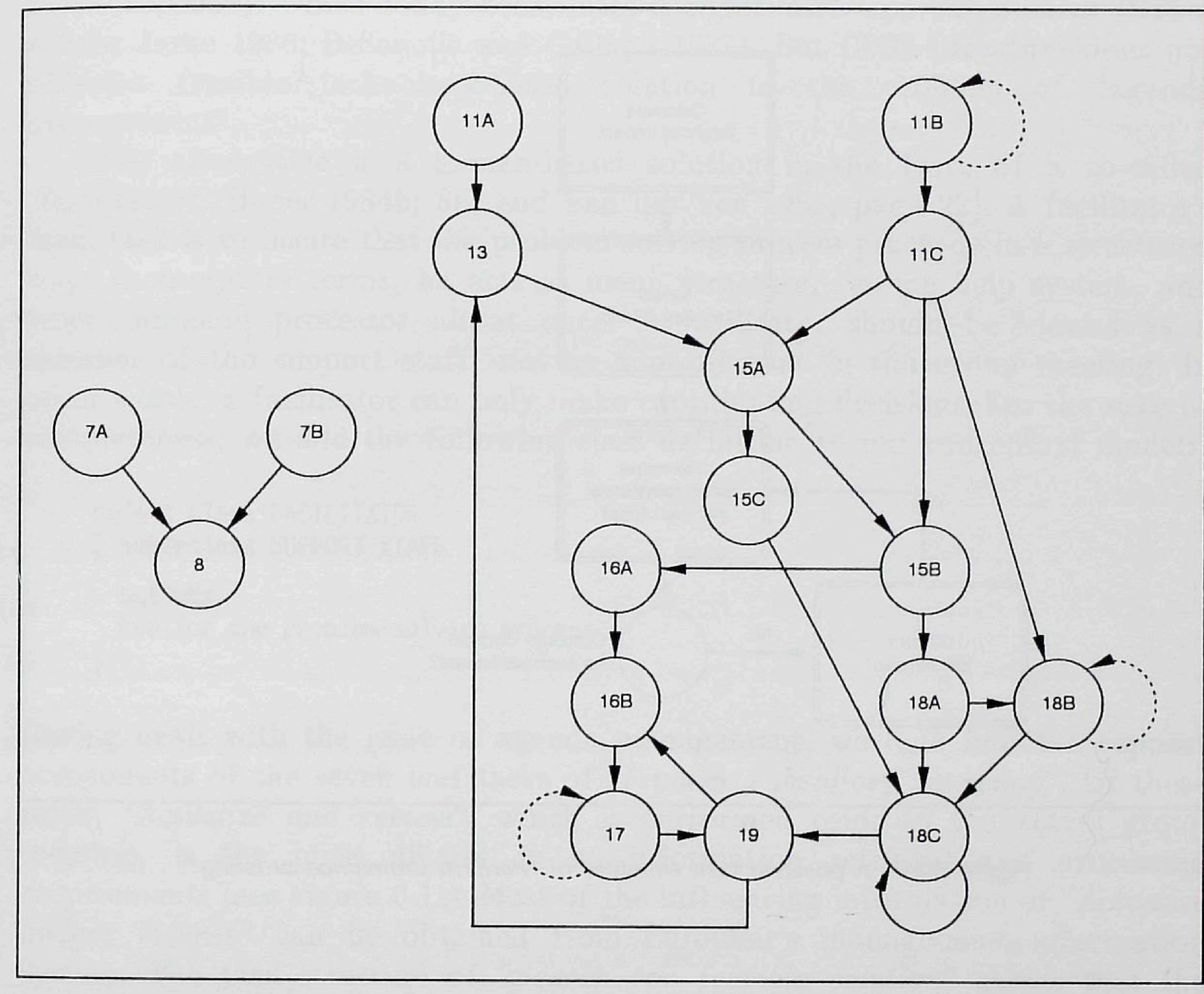

Flgure 6-20. The condensed decision structure of "Finance subsidiary company"

The single-node cycles around 11B, 17, and 18B do not indicate a coordination problem, since the auto-precedence is caused by the fact that these decisions involve an update of a specific information item. The cycle around decision $18 \mathrm{C}$ ("Determine actual remittance"), however, does indicate a coordination problem since there is no real update: The participants each define their "personal" actual remittance, and subsequently these are discussed. This coordination problem could for example be solved using the coordinating mechanism (flow relation plus coordinating decisions) depicted in Figure 6-21. Each subtask in this flow diagram contains one decision from Figure 6-20. 


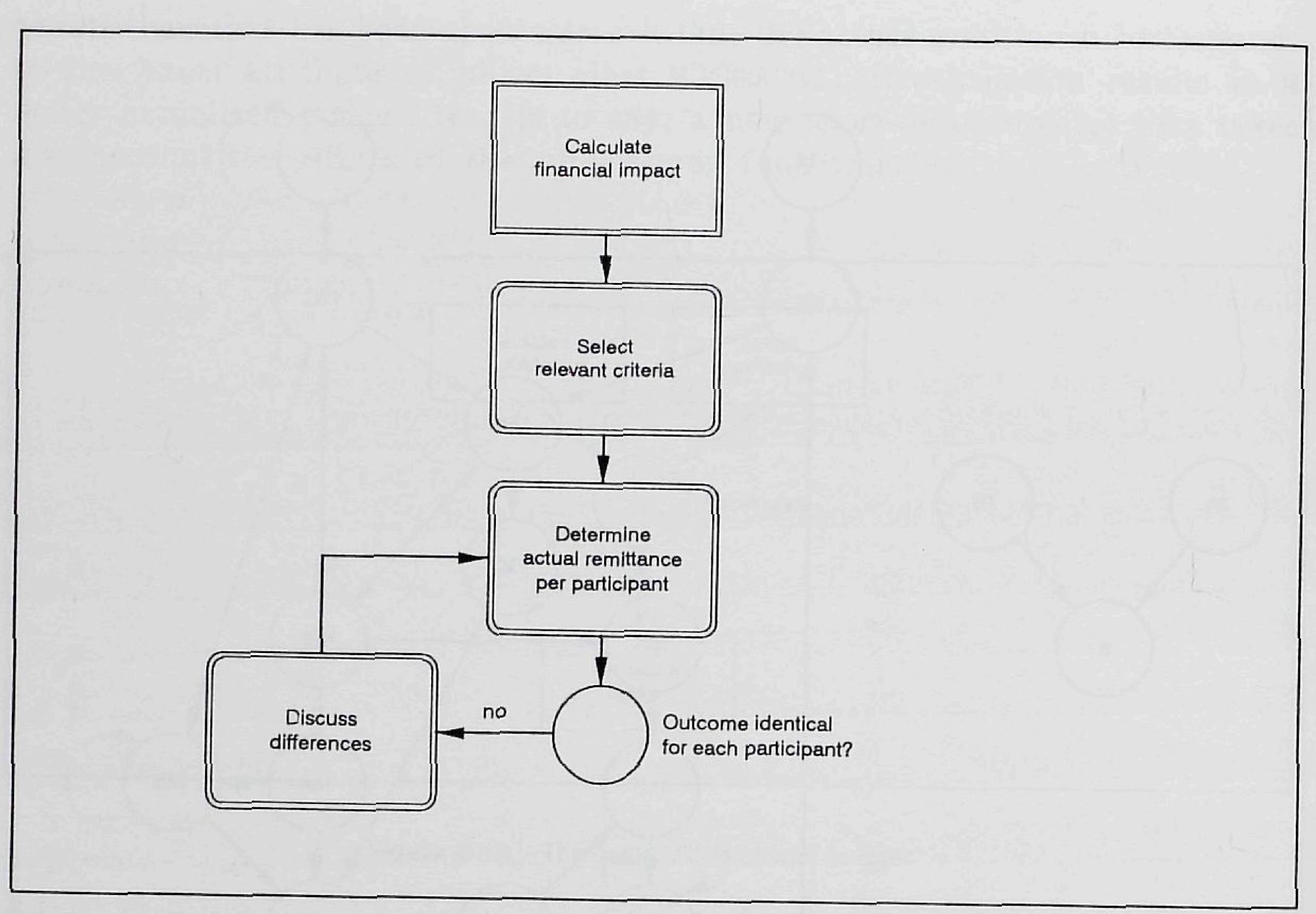

Figure 6-21. A possible task structure of "Perform preference ordering"

\subsection{DECISION SUPPORT}

The main difference between the subsidiary financing task and the shiftwork planning task analyzed in Section 5.4 is the number of decision makers that are involved. In Chapter 5 we could focus on the activities of one personnel manager, whereas now we have to ccordinate the actions of some five or six participants in a group meeting.

A second important difference between the subsidiary company financing task and the shiftwork planning task lies in the level of detail in which the actions of the decision maker(s) can be specified. Each action defined by object class PERSONNEL MANAGER can be translated directly in terms of changing certain codes in a team roster. The actions defined by object class PARTICIPANT are decidedly less concise.

Both differences make it difficult to implement an adequate agenda mechanism for the execution of the programme specified by the task structure of "Finance subsidiary company". The idea of computer-mediated group decision processes (Joyner and Tunstall 1970) has evolved into group decision support systems (GDSS), which today constitutes a significant area of research (Huber 1984b; Jarke 1986; DeSanctis and Gallupe 1987). But GDSS literature does not offer a feasible technology-based solution to the problem of "agenda management".

The alternative is a human-based solution in the form of a so-called "facilitator" (Huber 1984b; Sol and Van der Ven 1988, page 22). A facilitator's main task is to insure that the problem-solving process proceeds in a structured way. In computer terms, he acts as menu structure, on-line help system, and voice-command processor all at once. A facilitator should be viewed as a member of the support staff, not as a participant in the group meeting. In other words, a facilitator can only make coordinating decisions. For the sake of completeness, we add the following class definition to our conceptual model:

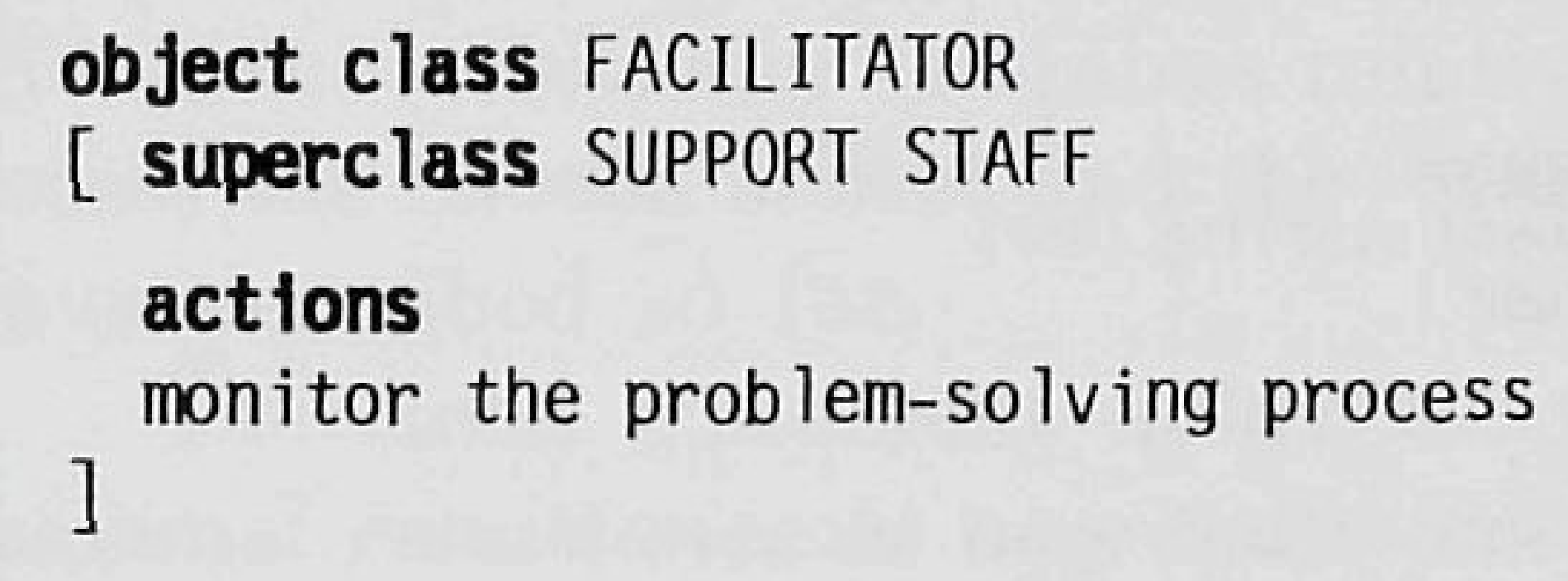

Having dealt with the issue of agenda management, we look into the support components of the seven leaf tasks of "Finance subsidiary company". Of these tasks, "Actualize and review", which is performed prior to the actual group meeting, is the most diverse in its information retrieval and processing requirements (see Figure 6-11). Most of the influencing information of "Actualize budget figures" can be obtained from Europhar's management information system. The task structure of "Search for feasible solution" shows that the actualized budget itself is also used when other tasks are executed. Since budget figures will be recalculated repeatedly during the feasible solution search, the budget itself can best be stored as a matrix in some spreadsheet-like application.

The review of all financing alternatives also requires a financial modeling tool. The costs of the different fund transfer alternatives is an important factor. These costs may be significantly affected by tax and duty rates. To compare the cost of various ways to transfer funds, calculation schemes like the one in Figure 6-22 can be used. The upper part of this scheme shows the relevant attribute values of the two companies that are involved (figures of 1988). The lower part shows how the financial impact of a particular fund transfer alternative is calculated. Recall that by "financial impact" we mean the marginal effect of a net liquidity increase (or decrease) of 100 local currency units (here GBP), expressed in the currency unit of the parent company (here NLG). Figure 6-22 shows that a transfer price increase of GBP 153.85 is needed to realize a liquidity decrease of GBP 100.00 , since higher transfer prices mean higher cost, and consequently reduce corporate tax in the United Kingdom. At the given 
exchange rate of $3.66 \mathrm{NLG} / \mathrm{GBP}$, transfer prices must be increased with NLG 563.09 , which results in higher revenues - but also higher corporate tax - for the parent company. Thus, the net effect is NLG 326.59, which is less than $3.66 \times$ 100 GBP. The difference of NLG 39.41 could be seen as the marginal cost of this way of transferring funds.

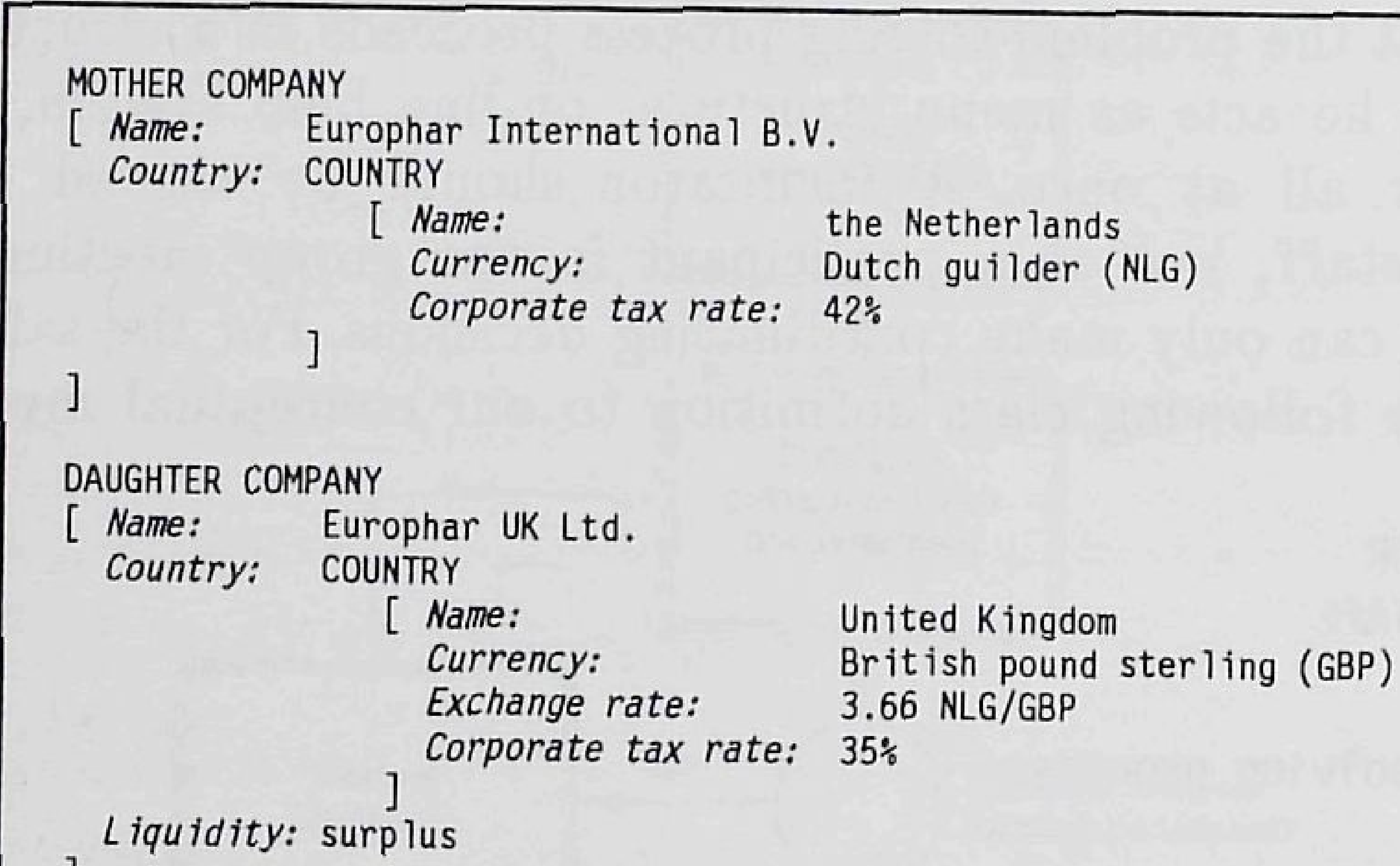

\section{Calculation of the marginal effect of a net liquidity decrease of GBP 100.00.}

\section{Liquidity decrease:}

UK corporate tax:

Transfer price increase in GBP:

Transfer price in NLG:

NL corporate tax:

Net increase of NL liquidity:

$\begin{array}{rr}\text { GBP } & 100.00 \\ + \text { GBP } & 53.85\end{array} \quad$ (35\% of $\left.153.85=53.85\right)$

Flgure 6-22. Financial impact of a transfer price increase

The third decision in "Actualize and review" is not so readily supported by means of information technology. While defining the object class BACKGROUND INFORMATION ITEM on page 141, we pointed out that the kind of information can vary widely, which makes it difficult to represent in some form other than that of a verbal document.

Since it is influenced by "Actualized budget", the single decision in leaf task "Define targets" (see Figure 6-13) can be supported by means of the budget worksheet mentioned earlier. The other influencing information is difficult to express in terms of "hard" figures and equation-type rules, which again points in the direction of a textual representation without any model component.
Progress in the area of for instance legal databases may eventually lead to more sophisticated support. By contrast, the financing, commercial, and fiscal targets can be expressed in terms of desired profit and loss, liquidity, etcetera. These figures should be stored in a worksheet that can be linked with the budget worksheet as to allow comparison.

A similar worksheet application could be used to record the "transient targets" that are set when "Select subset of alternatives and set total remittance" (see Figure 6-15) is executed. Similar to the previous decision, "Set 'transient' targets" is influenced by the regulations and the financing, commercial, and fiscal targets. But since it is also influenced by the six categories of fund transfer alternatives, these must be available as well (including their background information, financial impact, etcetera). This information must also be available when the decision "Select subset of alternatives" is made. Finally, the decision "Set total remittance" requires relatively little support. The decision makers can access the chosen subset and the "transient" targets using the support objects we have described so far.

The same statement holds for the task "Select alternative and determine maximal remittance at aggregate level". This leaves us with two more leaf tasks to support: "Disaggregate" and "Perform preference ordering". The former mainly requires access to a database that contains all relevant information on the articles that are manufactured by the parent company and the subsidiary company. We must emphasize that the selection of suitable candidates for a transfer price change is not a straightforward matter. Numerous complications are discussed in detail by Van der Ven (1989a, page 29-43).

As the decision structure in Figure 6-18 indicates, the financial impact worksheets should also be used during "Perform preference ordering". Our description of this task on page 160-161 shows that preference ordering is a multi-criteria decision process. Multi-criteria decision making can be supported by a wide range of techniques of varying degrees of complexity (Zeleny 1982; Roy 1985). Since the preference ordering task is performed during the meeting, a supporting technique should be readily understood by all participants, take up only a limited amount of time, and offer a good point of departure for discussion (Lootsma et al. 1986). For these reasons, the relatively simple technique described by Van der Ven (1989a, page 54-56 and 102-104) is most appropriate.

All of the support mentioned in this section, with the exception of the heuristic search at the disaggregate level, can be implemented with the help of a commercially available financial modeling tool. Since the details of this implementation bear little relevance to the purpose of this case study, we refer to Van der Ven (1989a) for a comprehensive report. 
We wish to conclude this section with the observation that neither the "executive feel" of an elegantly furnished decision room - favored by for example Gray (1986, page 163) - nor the very sophisticated technology described by Vogel et al. (1988) are prerequisites for effective decision support. A regular meeting room, two personal computers fitted with monochrome LCD overhead projector adapters, two projection screens (since the financial impact worksheets require the entire screen, a second computer with public screen is required to avoid tedious switching between applications), a flip chart, and a low-noise printe provide effective support at a low expense. Figure 6-23 shows the layout of the "improvised" decision room that has been used at Europhar.
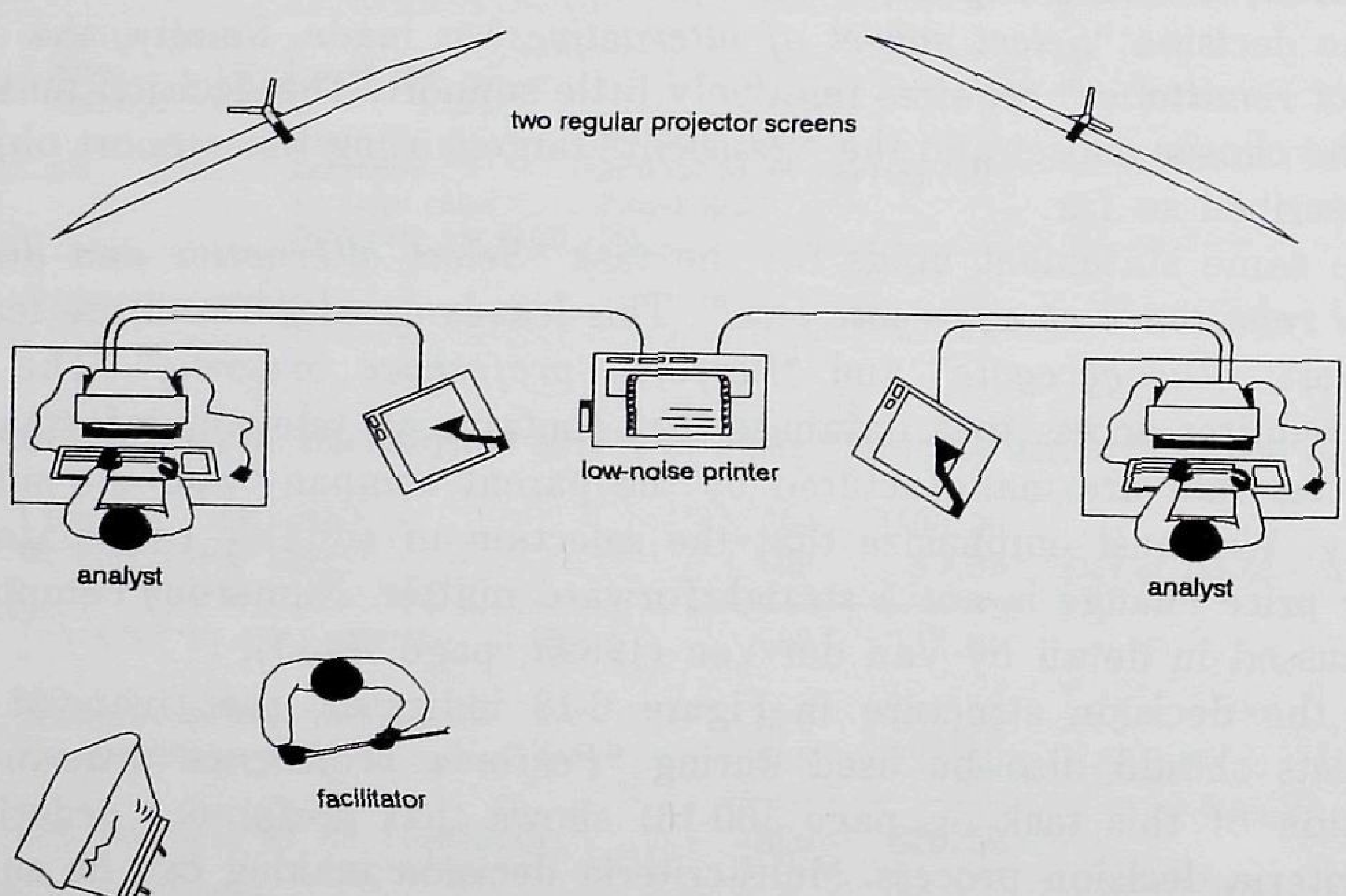

flip chart

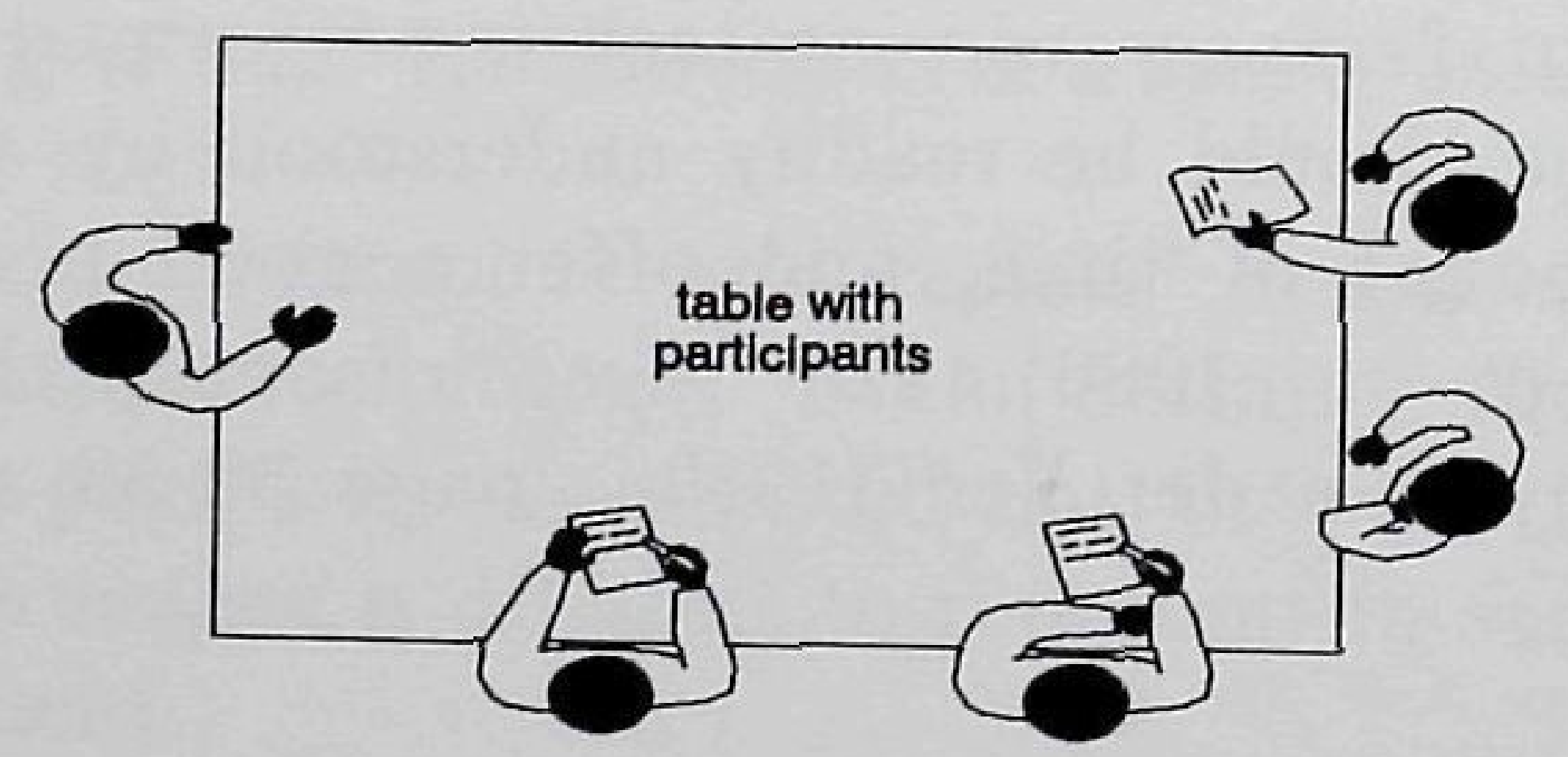

Fgure 6-23. Layout of an "improvised" decision room

\subsection{SUMMARY AND CONCLUSIONS}

In this chapter we reported on our analysis of the problem-solving process of a group of decision makers acting as a team. We presented a fairly detailed conceptual model of the fund transfer problem, and specified a problem situation that has been observed in reality. We demonstrated how task analysis can be used first to specify the problem-solving process as it takes place within the organization in order to get an understanding of that process, and later to design a programme that ensures a more structured and comprehensive approach to solving international fund transfer problems.

Like in Chapter 5, we discern two categories of conclusions. The first category relates to our approach to problem solving in general, and is based on our experiences with object-oriented modeling and our task analysis technique. The second category relates to our solution of the problem of international transfer pricing in particular.

I. The problem-solving approach

A first conclusion is that problem conceptualization in terms of objects, attributes, and actions is useful even if greater part of the actions cannot be specified as an algorithm. When modeling the fund transfer problem situation it was important to know that problem solving is largely performed in a group session in which participants exchange information on alternatives by bringing up and commenting on various ways to transfer funds, and analyze and evaluate these alternatives. This knowledge could be represented in the object class definitions. The question of how the participants performed these actions (and later the question of how they should perform them) can be answered by means of task analysis.

This leads us to the second conclusion, which is that our task analysis technique can also serve in situations where there is more than just one decision maker. Coordinating decisions, in particular those involved in signal constructions, proved to be quite adequate means to solve the coordination problem, even though task analysis does not provide means to assign specific decision makers to specific tasks.

Thirdly, we can conclude that task analysis of the transfer pricing problem has led to new ideas concerning both the group decision process and the possible use of computerized support.

Finally, we found that task structures with their obvious graphical representation and non-restrictive use of natural language to label flows are readily understood even by non-computer experts, thus constituting an effective means of communication between the decision makers, the analysts/designers, and the builders of the support component. 
II. The problem of international transfer pricing

We have shown that in the fund transfer problem situation both the preparatory tasks and the tasks performed during the group meeting can be structured to a large extent. For most of these tasks adequate computer support can be developed using a commercially available financial modeling tool.

We find that there is a large overlap between the support components of the leaf tasks, wich means that the support component alone provides little or no guidance in the problem-solving process. The importance of this guidance is confirmed by the fact that the contribution of the problem specification, the task structure, and the facilitator role obtained the highest ratings in the evaluation of the entire project (Van der Ven 1989a, page 146).

\section{ORGANIZATIONAL PROBLEM SOLVING SUPPORT}

\section{THE CASE OF A MANAGEMENT GAME}

A very exciting game that's played all over the world. Good players get rich, and bad players don't. - David Eddings

\subsection{INTRODUCTION}

Our third case study focuses on organizational problem solving. This means that we shall be looking at a fairly large collection of decision processes that take place concurrently within an organization. The primary purpose of this particular case study is to test whether our task analysis technique can be applied successfully to a collection of problems that are entwined and yet solved at different places in an organization. Its secondary purpose is to study the "adaptivity" of task structures, that is to say, we want to investigate how easily a specific task structure can be modified when changes occur in the problem situation for which it was designed.

The problem we studied is the management of a simulated factory, based on the total enterprise management game VUMAS (Rematch 1987). In this game, a number of competing chemical companies produce and market up to five products. Each company is run by a team of three to five players. Time is simulated by playing rounds, where each round corresponds to a game period of three months. During a round of $1 / 2$ to $1 \frac{1 / 2}{2}$ hours the following decisions have to be made:

- purchase quantities of four kinds of raw materials directly from the suppliers;

- initiate one-year contracts with the suppliers to ensure a steady supply of raw materials at a fixed price;

- for two production stages, expand or reduce the production facilities and determine the size of the work force;

- decide on wage level, training, and work planning;

- for each of the five products, set production levels for the next quarter;

- purchase market information;

- for each of the five products, set selling price and determine quantities to offer on the market;

set total advertising expenditure

- borrow short-term or long-term funds;

- determine dividend to be paid to shareholders;

- pledge for government support 
The choice of this particular case was based on the following considerations:

Relevance

In Chapter 5 and 6 we stated that we prefer problems of present interest and of a general nature. From this vantage point the relevance of solving an "artificial" problem like that of playing a management game can be questioned. Nevertheless, we find that other factors (explained in more detail below) in this case outweigh relevance.

\section{Demarcation}

The specific problem had to require the performance of a variety of interdependent tasks by different individuals or groups. Preferably, it should require both strategic and operational decisions to be made. The VUMAS management game meets both demands. The relatively short (one-hour) rounds absolutely require a division of tasks among the players of a team, which introduces a fair-sized coordination problem. Investment decisions can be classified as strategic decisions, while purchase and production planning are obvious examples of operational decisions.

\section{Complexity}

The problem situation should again be complex in the sense that it surpassed the cognitive ability of the decision makers. The problem-solving process should be heuristic, rather than algorithmic in nature. Moreover, since we wish to test our task analysis technique in a situation that requires coordination over different moments in time, the problem should have an explicit time dimension. On the other hand, the problem should not be too complex. The tremendous amount of details involved in day-to-day management would be obfuscating. A management game abstracts from those details, while retaining the structure of the problem: multiple, concurrent problem-solving tasks. The flow of goods in a VUMAS company follows the classical "purchase-stock-productionstock-sales" pattern. Production has to be planned in the period before it take place, while products cannot be sold until one period after they have been manufactured. This introduces some interesting planning problems.

\section{Structuredness}

Similar to our previous two cases, the problem had to be ill-structured in the sense that no formalization of the problem-solving process had taken place so far. In Section 4.2 we distinguished between "known" and "unknown" problem situations (see Figure $4-12$ on page 63). We argued that if a problem is unknown, that is to say, if it has not been solved before, it is easier to start with making a decision structure than a task structure. The personnel planning problem and the daughter company financing problem both classified as "known" problems. They illustrated the sequence "describe task-analyze task structure-improve task structure". To novice players, the total enterprise management problem is unfamiliar. As such it will be a good illustration of the "describe decisions- analyze decision structure-make task structure" approach. The decisions mentioned on page 171 have been identified in advance, and therefore are known to the players. However, the VUMAS users manual offers no explicit guidelines with respect to how these decisions should be made under specific circumstances. It does not give any hints on appropriate coordinating mechanisms or on operational or long-term strategies. Needless to say, game parameters like elasticities and seasonal fluctuations are not disclosed either.

Field test

In the previous two chapters we stated that for our purpose the problem situation had to be recurring as well as "real-life" in the sense that organizations could be found within which proposed solutions and supporting technology could be tested using company data. The simulated time of a management game makes it possible to perform these tests within a relatively narrow time frame. In addition, a management game like VUMAS has the advantage that problem situations can be created at will. This makes a study of the adaptivity of task structures possible.

These considerations lead us to believe that a management game can very well serve as a case study on organizational problem solving. Basically, the players of the game face the problem to maximize their own profit, while maintaining a healthy company. Our decision to use the VUMAS management game in particular is based on the results of a comparative review by Van Schaik (1986) who used the game to test the effectiveness of a specific spreadsheet-based decision support system under laboratory conditions (Van Schaik 1988).

In this chapter we shall again follow the problem-solving approach we described in Chapter 3. In Section 7.2 we describe the conceptualization activity, at the same time disclosing more details of the simulated firm. The empirical model is specified in Section 7.3.

We then get to the essential part of this chapter. In Section 7.4 we demonstrate that a gaming strategy can be found by applying task analysis to the problem stated in the users manual. We show how the identification and analysis of decisions eventually leads to a task structure that specifies which management tasks have to be performed, and how they should be coordinated.

In Section 7.5 we describe how these management tasks can be supported by means of information technology, and how this support has eventually been implemented. In the course of our research we simulated and analyzed numerous "crises", varying from a sudden shortage of one kind of raw materials to a total strike. To illustrate the adaptivity of task structures, we report on one of these crises in Section 7.6. We end this chapter with a summary of this case study and an overview of our conclusions. 


\subsection{CONCEPTUALIZATION}

Like we did in the previous two chapters, we commence by identifying the object classes in terms of which the problem situation can be described. The fact that we are dealing with a game situation simplifies the conceptualization task considerably - a 50-page users manual is easier to analyze than a real-life multi-million organization! However, this does not mean that the identification and specification of object classes is entirely straightforward. In the course of this section we shall point out how specific choices with respect to classes and attributes have been made.

As usual, we first identify the objects that are "directly visible", such as companies, workers, and products. More abstract notions like "loan" and "profit" are more easily defined once the concrete objects have been described. We start by defining the largest object: the company. For this particular problem we need a much more detailed definition of object class COMPANY than the one that is given in the previous chapter.

We emphasize that this new definition of COMPANy has evolved over time. The one on the next page is the final version. The choice of attributes is partly the result of the way we demarcate the boundaries of the problem situation, and partly a matter of interpretation.

The most important decision we made during the conceptualization activity is that we view a team of players of the game as the IS-component of the problem situation, and their company as the corresponding RS. The demarcation rule limits the attributes we can identify. Since the IS must control the RS, object class COMPANY cannot have attributes that are beyond the control of the players. But the demarcation rule does not impose one particular representation of a company. And, as we already stated on page 8 , neither does object-oriented
modeling.

We identified the stock level of raw materials a an attribute of COMPANY rather than of RAW MATERIAL, since the stock level can be controlled by the company, while the other aspects of raw materials, such as name and price, are part of the environment. A company's production facilities are modeled as instances of INSTALLATION and BOILER. The players are concerned with only their own production plant, so both classes can be seen as part of the RS. The same argument holds for contracts, warehouses, and loans. Products are a different matter. We could define an object class PRODUCT to refer specifically to the players' products, in which case it would have attributes like Selling quantity and Selling price. With this definition, other companies' products (their prices and supply) play an important role in the players' problem situation. modeled as other instances of PRODUCT, but that would make it difficult to classify PRODUCT as part of either the RS or the environment.

\section{bject class COMPANY}

attributes

Raw materials in stock

Contracts

Installation

Boilers

Crewing system

Installation operators Boiler operators Wage level

Gratuities

Inventory

Selling quantities

Selling prices

Advertising budget

Loans

Share capital

Liquidity

actions

buy information

buy raw materials directly

initiate a new contract

expand/reduce main installation

buy/sell boilers

change number of crews

hire personnel

fire personnel

negotiate with unions

change wage level

plan production

manufacture products

change advertising budget

set selling prices

set selling prices

apply for a loan

pay interest on a loan

repay a loan

pay a loan

declare dividend
A list containing for each kind of raw material the quantity in kilograms that is currently in stock.

A list containing for each kind of raw material a contract, if one exists.

A A main installation, used for the first production stage. A list containing this company's operational boilers The crewing system according to which the main installation unit is run

The work force for production stage 1

The work foce for production stage II.

Base salary for installation operators is NLG 4000, for boiler operators NLG 5000 per period. The wage leve pid. Thus, if page level equat 105, the act is actually would be NLG 4200 and NLG 5250, the actual salaries The sum of the various gratuities awarded to the pers The sum of the various gratuities awarded to the personne
on top of their wages, expressed as a percentage of the

Represented

warehouse containing the current stock of raw materials and finished products.

A list containing for each market the quantity of product (in kilograms) to be offered on that market.

A list containing for each market the price (in NLG per kilogram) for which the product should be sold.

The amount in NLG spent on advertising for the product Abyco.

A list containing the loans this company has at a bank. The total nominal value of outstanding stock. Dividend is declared as a percentage of this sum.

The current liquidity of this company, expressed in NLG. 
An alternative is to view a product as a more abstract object, defining only the composition in terms of raw materials. Selling quantities and prices are in this case modeled as attributes of COMPANY. This resolves the issue of classifying PRODUCT as an RS-object or environment object. Moreover, installations and boilers are discrete entities, but the chemicals produced are bulk products. We find that the "abstract" definition of PRODUCT is closer to the actual situation. Both considerations made us choose for this second representation. Note that this decision is a matter of interpretation. It is not enforced by the demarcation rule, nor by object-oriented modeling conventions.

The COMPANY class definition covers about every player action of the game that can be found in the users manual. By simply scanning the list of attributes and actions, we can identify other object classes for our conceptual model.

$\begin{aligned} & \text { object class RAW MATERIAL } \\ & \text { [ attributes }\end{aligned}$
$\begin{array}{ll}\text { Name } & \text { The name of this raw material. } \\ \text { Current price } & \begin{array}{l}\text { The price for which this raw material can be purchased in } \\ \text { the current period. }\end{array} \\ \text { Accounting price } & \begin{array}{l}\text { The price for which this raw material appears on the balance } \\ \text { sheet. }\end{array} \\ ]\end{array}$

The markets for raw materials are highly dynamic. Prices vary strongly under influence of structural, conjunctural, seasonal, and random fluctuations. For this reason, an accounting price is used in determining the value of a company's stock of raw materials.

Raw materials can be purchased either directly, for the current price, or on contract. For each kind of raw material, one-year contracts can be initiated. When buying on contract, a company orders a large quantity (20 tons minimum) in period $p_{0}$, and one-fourth of this quantity will be delivered in the periods $p_{1}, \ldots, p_{4}$ at a fixed price, namely that of period $p_{0}$. A contract can be described using the following object class:

\section{object class CONTRAC \\ [ attributes \\ Fixed price \\ Remaining quantity \\ Remaining periods \\ The price per kilogram of the period in which this contract was initiated. \\ The total quantity that still has to be delivered. \\ The number of periods over which this quantity has to be \\ spread out.}

Once initiated, a contract cannot be cancelled or changed, nor can there at any time exist more than one contract for the same kind of raw material. Payment is due upon delivery. If more than 40 tons of one kind of raw material is delivered in a period, the company gets a $2 \%$ discount over the entire quantity, regardless whether this delivery resulted from direct or contractual purchase, or some combination of both.

Next, we identify the object class PRODUCT. Each product has a specific name and composition. Every company is at liberty to set its own selling prices, as long as they remain within certain limits, and observe some other conditions we shall specify in the next section. To preclude flattering balance sheets - products are priced by the company - one fixed accounting price is set for each product. Thus, a stock of $x$ tons of product $P$ will always appear for the same value on a balance sheet, regardless of the current selling price of that product.

\section{object class PRODUCT}

[ attributes

Name

Composition

Minimum price

Maximum price

Accounting price

]

The name of this product.

For each kind of raw material the quantity required for the production of 1 kilogram of this product.

The lowest price in NLG per kilogram for which this product can be sold.

The highest price in NLG per kilogram for which this product can be sold

The price in NLG per kilogram for which this product appears on the balance sheet.

Note that the current selling price and stock level are not modeled as an attribute of PRODUCT. Adding these attribute could be defended from a onecompany perspective, but competing firms may set different prices and have different stock levels - an issue we raised while specifying COMPANY.

Production takes place in two stages. The first stage consists of mixing appropriate quantities of raw material, solving it in water, and heating it to approximately $85^{\circ} \mathrm{C}$. This process requires a large installation, represented by the following object class:

\section{object class INSTALLATION}

\section{attributes}

Production capacities

Manning requirements

Fixed costs

Variable costs

Expansion costs
The maximal production capacity (in tons per period) of this installation when operated by 1,2 , and 3 crews, respectively. The crew size required to run this installation.

The fixed costs of using this installation, expressed in NLG per period.

The variable costs of using this installation, expressed in NLG per kilogram product.

The price in NLG of an increase of this installation's production capacity by $15 \%$. 
An installation can be operated according to a 1-crew, 2-crew, or 3-crew crewing system. If necessary, the production capacity of an installation can be increased. Such expansions do not require additional personnel.

In the second production stage, the heated solution is transferred to one or more boilers. A boiler heats the mixture to some $120^{\circ} \mathrm{C}$ for quite some time, during which the chemical reaction that results in the final product takes place. Boilers are operated according to a 3-crew system similar to that on page 100 . The manning requirements of a boiler depend on the type of product being manufactured. Production capacity is expressed in boiler hours, rather than tons. The actual production volume in tons depends on the series length. By "series" we mean a stretch of time in which a boiler manufactures one kind of product. Long series require less cleaning and adjusting time, resulting in a better yield.

\begin{tabular}{|c|c|}
\hline Boiler hours & $\begin{array}{l}\text { The maximal number of hours this boiler can operate during } \\
\text { one period. }\end{array}$ \\
\hline Production capacity & $\begin{array}{l}\text { The relationship between boiler hours and yield for this } \\
\text { boiler, expressed for each product in tons. }\end{array}$ \\
\hline Manning requirements & $\begin{array}{l}\text { A list containing for each product the number of workers } \\
\text { required to operate this boiler. }\end{array}$ \\
\hline Fixed costs & $\begin{array}{l}\text { The fixed costs of using this boiler, expressed in NLG per } \\
\text { period. }\end{array}$ \\
\hline Price & The price of a new boiler. \\
\hline Planning & $\begin{array}{l}\text { A list containing for each product the number of hours this } \\
\text { boiler should process that product. }\end{array}$ \\
\hline
\end{tabular}

The object classes INSTALLATION and BOILER cover the technical side of the production. Next, we consider the human resource. Individual workers could be described using the object class defined in Chapter 5 on page 93, but since the VUMAS game does not require decisions to be made with respect to individual workers, we define work forces instead:

$\begin{aligned} & \text { object class WORK FORCE } \\ & \text { [ attributes }\end{aligned}$
$\begin{aligned} & \text { The number of workers in this work force. } \\ & \text { Standard wages }\end{aligned}$
$\begin{aligned} & \text { The base salary (in NLG) for the workers in this work force. } \\ & \text { The costs (bonuses, moving expenses) that have to be made } \\ & \text { to add one worker to this work force. } \\ & \text { The percentage of workers that is absent due to illness } \\ & \text { etcetera. }\end{aligned}$

\section{actions}

strike

]

Hiring costs and absence level depend not only on a company's wage level and gratuities, but on the conditions at other companies as well.

Unlike workers, the unions require a more detailed specification:

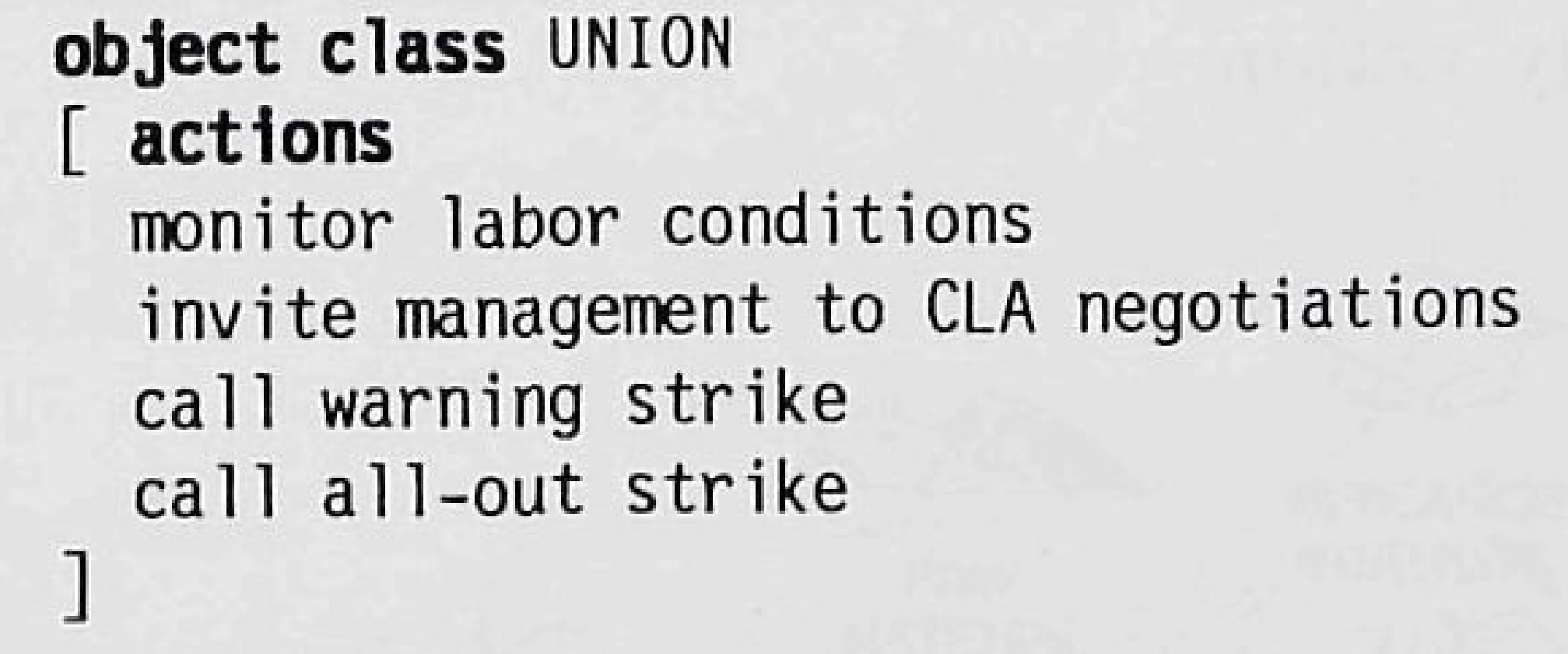

As is pointed out by Van Schaik (1988, page 66), VUMAS differs markedly from management games in the United States with respect to the management of personnel, a result of the differences that exists between the Dutch and American economy in this regard. For example, the consequences of lay-off in the Netherlands are quite severe. In almost every major management game of Dutch origin, poor personnel management leads to strikes, while striking personnel is virtually non-existent in most American management games.

Both raw materials and finished products are stocked in warehouses. Since the game puts no constraints on the warehousing capacity, we take the position that one instance per company suffices. We therefore define the following object class:

\section{object class WAREHOUSE \\ [ attributes \\ costs}

Raw materials in stock

Products in stock

]
The warehousing costs, expressed in NLG per kilogram per period.

A list containing for each kind of raw material the quantity (in kilograms) currently in stock.

A list containing for each product the quantity (in kilograms) currently in stock.

Products can be sold in the period following the one in which they have been manufactured. The markets for the various products are completely independent. The potential of a market depends on the selling price(s) of the product and advertising expenditures, but also on conjunctural fluctuations.

\section{object class PRODUCT MARKET}

[ attributes

Name

Product

Potential

Potential

]
The name of this market.

The product sold on this market.

The price/demand curve for Product on this market.

A list containing the companies that operate on this market. 
Since VUMAS companies are not allowed to give out more shares, banks are their only source of capital. Banks can provide both short term and long term oans, as well as "emergency loans". The latter are automatically provided if a company's cash assets fall below zero - a feature common among management games. Granting loans is a bank's only action.

\section{object class BANK \\ [ actions \\ grant loan}

Loans can be described in terms of the amount of money involved, the interest charged, and the moment a loan must be paid back.

\section{object class LOAN \\ [ attributes \\ Amount The size of this loan, expressed in NLG \\ Interest rate The interest rate for this loan, expressed as a percentage per period. ] \\ The period in which the loan has to be fully repaid.}

The government plays two distinct roles: as a taxing agency and as a supporting institution. In its first role it levies corporate tax - in this game $40 \%$ of each company's net annual profit, to be paid each spring. The government will only act in its second role if a company's survival is at risk and its personnel's jobs are in danger. In such situations the government may provide low-interest capital to make investments, but at the same time it will have the right to veto any action proposed by the company's management.

\section{object class GOVERNMENT \\ actions \\ grant support \\ veto actions of supported companies}

The last object class we wish to identify represents the research institute that - at a price - provides information to the players concerning both the raw materials markets and the markets for finished products. What kind of information can be obtained from this institute will be specified in the next section.

\section{object class RESEARCH INSTITUTE}

\section{[ actions}

investigate

]
Since no "loose ends" (attributes or actions that refer to unspecified object classes, or actions that refer to unidentified attributes) remain, we can say that we identified and specified all object classes required to describe the VUMAS problem situation. The next step is to determine its boundaries.

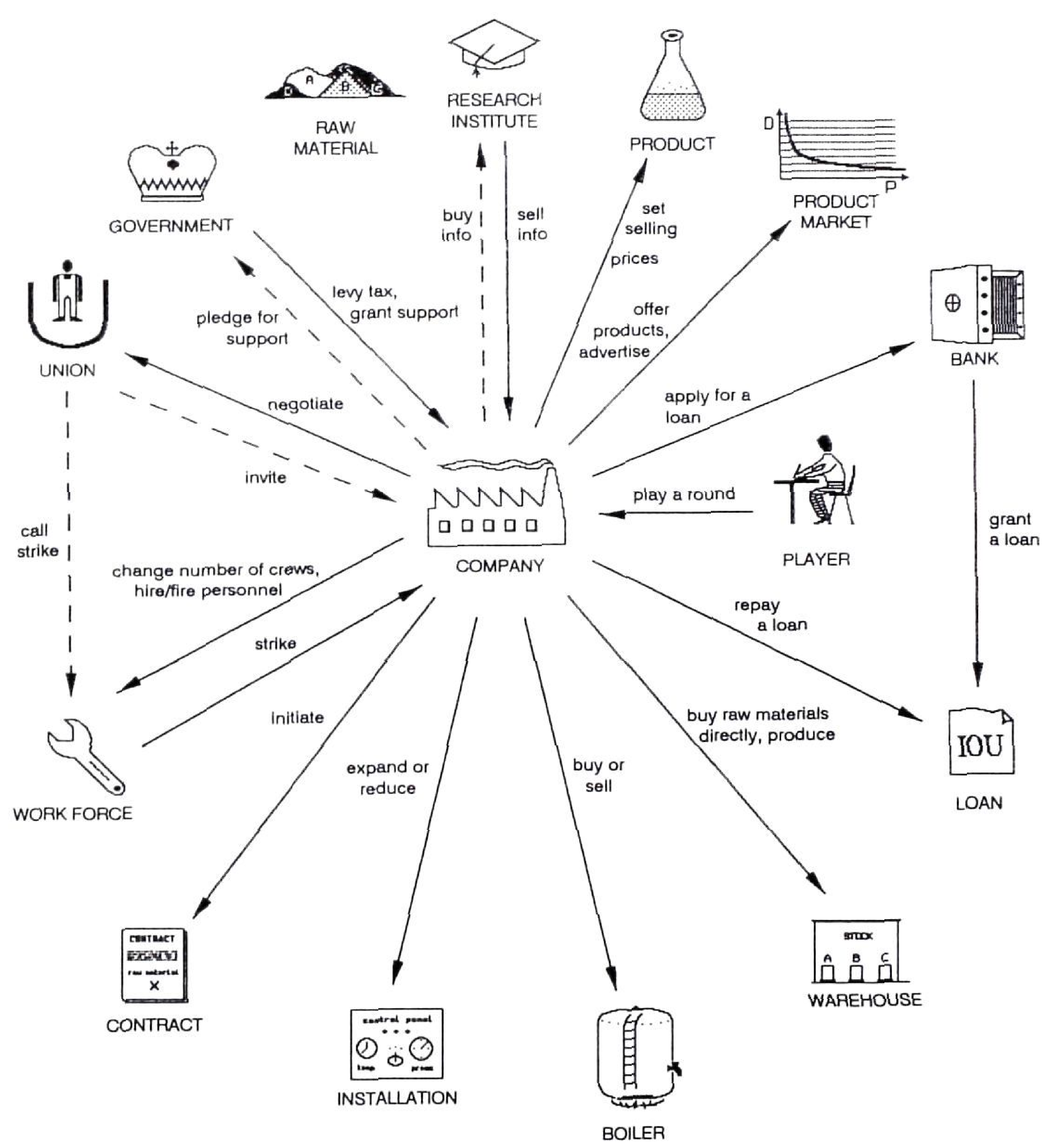

Fgure 7-1. A graphical representation of the VUMAS conceptual model

Again, we view the problem situation as an IS/RS-combination. In our discussion of the COMPANY class we already pointed out that we consider the players of the game (the "managers") to be the IS-component. After all, the players are the 
actual decision makers. Since they run their own company, this instance of COMPANY is part of the RS. The competition needs not be modeled as instances of COMPANY, since the actions of competing firms are reflected by the behavior of the markets, and by the information that can be obtained from the research institute. Object classes that definitely belong to the environment are RAW MATERIAL, PRODUCT MARKET, UNION, BANK, GOVERNMENT, and RESEARCH INSTITUTE, but also PRODUCT, since none of its attributes can be affected by the decision makers. Contracts, installations, boilers, work forces, warehouses, and loans can be considered as part of the RS, since only those objects that relate to the players' company will be represented by instances in the empirical model.

Figure 7-1 shows a graphical representation of the relations between the various object classes. A dashed arrow indicates that the corresponding action does not change the affected object directly, but rather induces the affected object to perform some action of its own. Observe how this graph reveals two flaws in our demarcation: The edge call strike from UNION to WORK FORCE, and the edge grant loan from BANK to LOAN indicate direct influences of the environment on the RS-component. The demarcation rule (see page 10) forbids such direct influences in a conceptual model because they introduce unnecessary equivocality. For example, in the current model it is unclear whether loans are a controllable or uncontrollable factor. The demarcation rule aids in eliminating this equivocality by demanding either that loans are viewed as an external factor (if the behavior of the bank is unpredictable, that is to say, beyond control of the decision makers), or that the bank is considered part of the RS (if the bank's behavior is predictable, for instance if loans are granted according to some known "algorithm"). Since the VUMAS users manual does not give objective criteria for granting loans, we chose to respect the demarcation rule by moving LOAN from the RS to the environment. UNION, on the other hand, becomes part of the RS, because a company can actually "control" the unions in the sense that it has very effective instruments to avoid or provoke a strike (wage level, gratuities, etcetera).

According to the users manual, the formal division of labor in the enterprise is pretty much standard: There is a Marketing \& Sales department, a Production department, a Purchase department, a Personnel department, and a Finance department. These departments can be viewed as five IS/RS-combinations. From this perspective, pricing decisions would for example be taken in the IScomponent of Marketing \& Sales. Figure 7-2 shows the demarcation of the five IS/RS-combinations. But we must keep in mind that they are subsystems of the system demarcated earlier - subsystems that must be coordinated. A decision to expand the installation or to buy a new boiler cannot be made by the Production or Finance department alone!

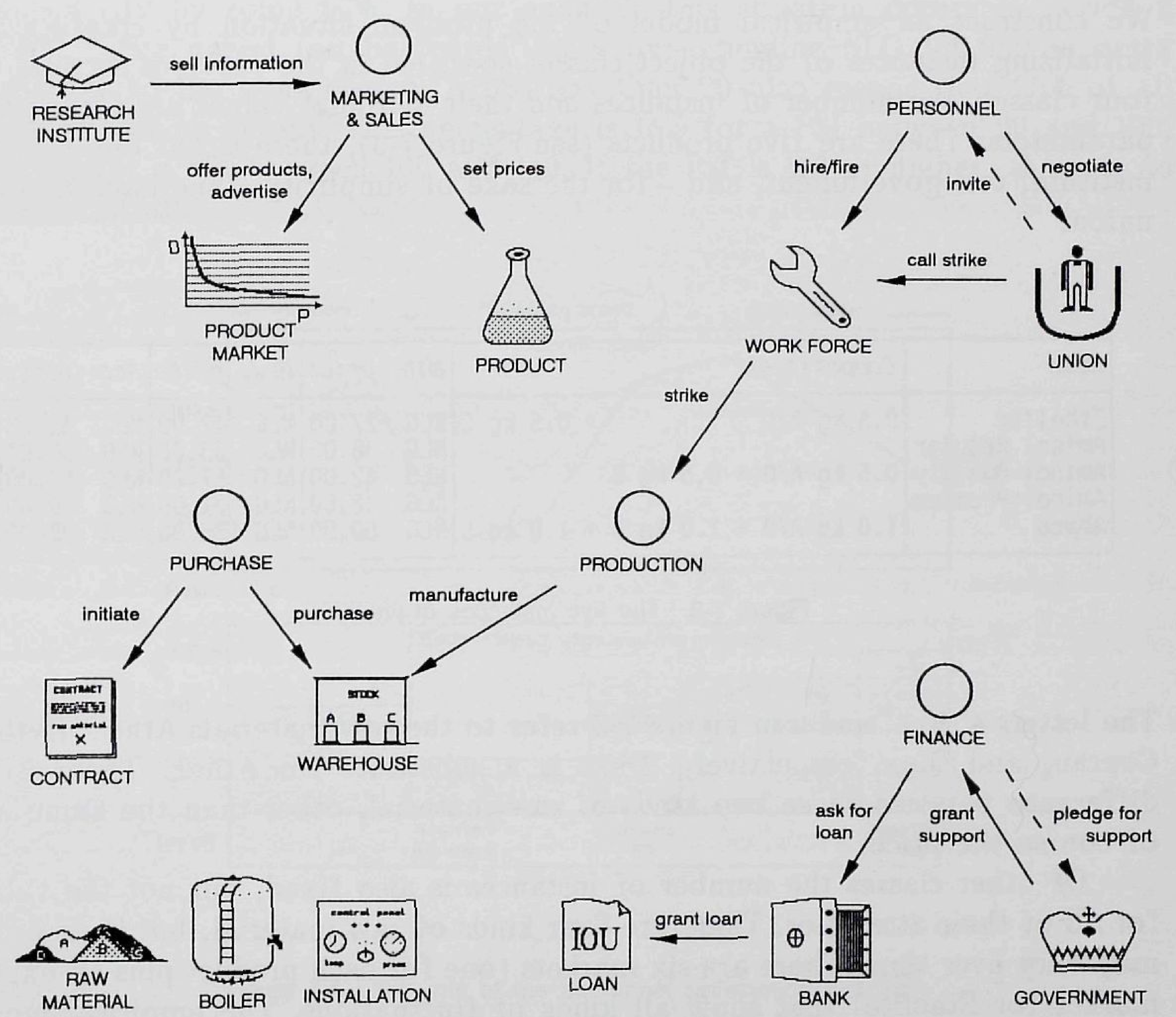

Fgure 7-2. The VUMAS conceptual model after applying the recursion principle

Note how Figure 7-2 immediately visualizes a number of coordination problems: WAREHOUSE is affected by both Production and Marketing \& Sales, and Personnel may cause a strike that will affect Production. Also note that INSTALLATION has become part of the environment since investment decisions are not made by any of the five IS/RS-combinations.

We emphasize that by applying the recursion principle of the information paradigm in this particular way, we merely implement the classic structure of a production firm. Further specification of the problem situation and subsequent task analysis will reveal whether this form of organization is appropriate in the sense that coordinating mechanisms can be realized with relatively little effort. 


\subsection{SPECIFICATION}

We construct an empirical model of the problem situation by creating and initializing instances of the object classes specified in the previous section. For four classes, the number of instances and their attribute values are fixed game parameters: There are five products (see Figure 7-3), there is but one research institute, one government, and - for the sake of simplicity - one bank and one union.

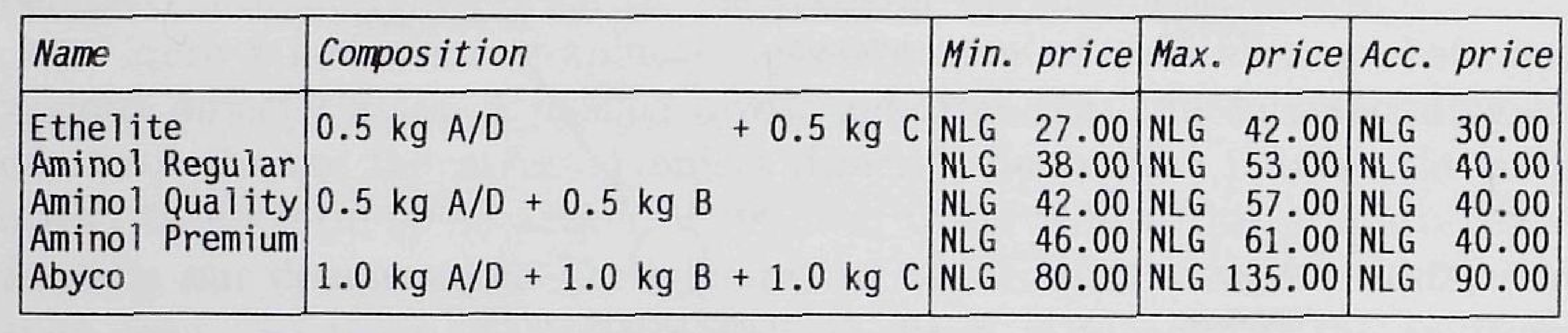

Figure 7-3. The five instances of PRODUCT

The letters A, B, C, and D in Figure 7-3 refer to the raw materials Athex, Bythol, Copran, and Diox, respectively. Diox is a substitute for Athex. There is no difference between these two kinds of raw material, other than the name and of course the price.

Of other classes the number of instances is also fixed, but not the values for all of their attributes. There are four kinds of raw material, but their prices may vary over time. There are six markets (one for each product plus an export market for Ethelite) that show all kinds of fluctuations. The empirical model contains only one instance of COMPANY and INSTALLATION.

A company starts out with three boilers, a work force of 50 installation operators (two crews of 25 workers), and one of 35 boiler operators. These number may change over time, but the attributes of a boiler maintain their initial values. Standard wages are NLG 4000 and NLG 5000, respectively, the initial wage level is 100 , and no gratuities have been extended over the past year.

In the previous section we mentioned that the absence level of the work forces depends on wage level and gratuities of the employing company and the competing firms. The reaction of the personnel to changes in these attributes are modeled using a so-called "personnel satisfaction indicator" (PSI), which is essentially an artefact in the game itself. The diagrams in Figure 7-8 give an impression of how various circumstances affect the PSI.

If the PSI is lower than the average PSI of all companies (as is the case in period 2, 3,6 and 7 of our example), hiring costs increase by NLG 1000 per newly hired worker. A PSI of 80 or less indicates labor unrest, which reduces productivity by some $50 \%$. In our example this situation occurs in period 2 and 3. Labor unrest can be "cured" only by extending NLG 100,000 in extra gratuities to the work forces. A PSI less than 90 also means that $25 \%$ of all workers will be absent. This percentage is $15 \%$ for a PSI between 90 and 100 , and $5 \%$ for a PSI between 100 and 110 . If the PSI is 110 or higher, there is no absence at all.
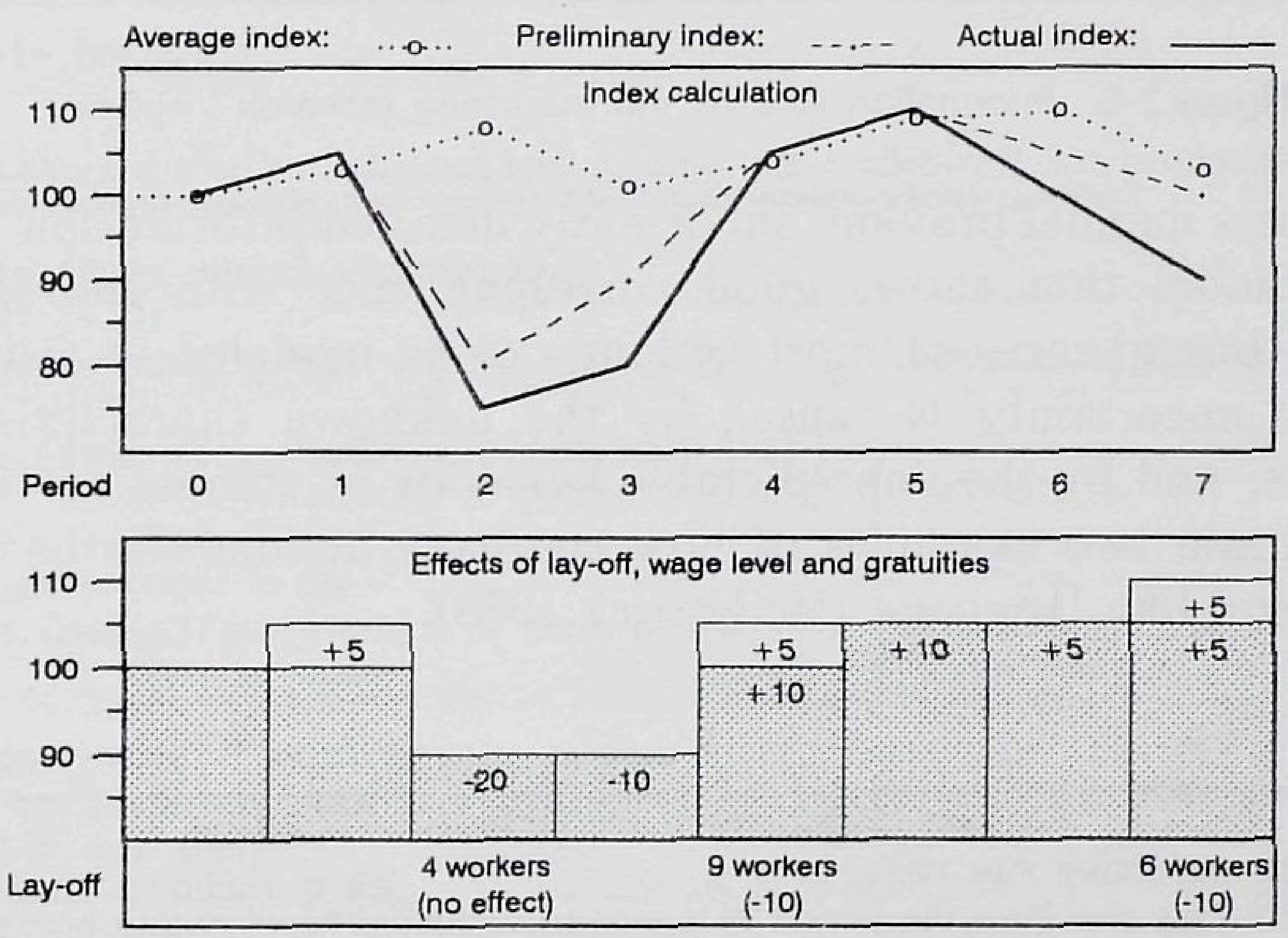

Figure 7-4. The role of the personnel satisfaction indicator

Note that the wage level affects the PSI both in absolute and in relative sense: preliminary $\mathrm{PSI}_{p}=$ Wage level $l_{p}-100+$ Wage leve $l_{p}-$ Wage leve $l_{p-1}+$ Gratuities $p_{p}+$ layoff $f_{p}$, where layoff $f_{p}$ equals -10 if five or more workers are fired in period $p$. The influence of the working conditions in competing companies is modeled by means of the following rule: If the preliminary PSI in period $p$ is less than the average of the preliminary PSIs of all companies, the actual PSI in $p$ equals the preliminary PSI minus 5 points. If this was the case in period $p-1$ as well, the actual PSI is lowered by another 5 points.

Figure 7-5 shows the kinds of information the research institute can provide. The VUMAS game imposes certain restrictions. Prices of raw materials in some period later than $p_{4}$ are not available. Moreover, each round a team can obtain for each product information on the market potential for only one period/price/ advertising budget combination. Thus, money can never buy an exact estimate of price/demand curves - a limitation we find realistic. 


\begin{tabular}{|l|l|}
\hline Information & Price in NLG \\
\hline Total actual sales (in tons) last period & $2,000 /$ product \\
\# of companies that operated on a market in the last period & $1000 /$ market \\
Potential (in tons) at a price P and advertising budget A & $10,000 /$ market/period \\
Idem, totalled over next 4 periods & $15,000 /$ market \\
Selling prices of competitors for all products in last period & 1,500 \\
Advertising budget of competitors in last period & 2,500 \\
Actual sales of competitors for all products in last period & 15,000 \\
Selling prices of raw materials & $7,500 /$ period \\
Selling prices of raw materials for next 4 periods & 22,000 \\
\hline
\end{tabular}

Flgure 7-5. Information sold by the marketing research institute

The VUMAS users manual provides sufficiently detailed information to construct an empirical model that shows good correspondence with the actual game situation. The consequences of most actions can be modeled in a deterministic way. The only uncertainty is caused by the unknown characteristics of the various markets, and by the unpredictable behavior of competing firms. Figure 7-6 and 7-7 contain two examples of how company actions can be specified in terms of an Algol-like language (McGettrick 1978).

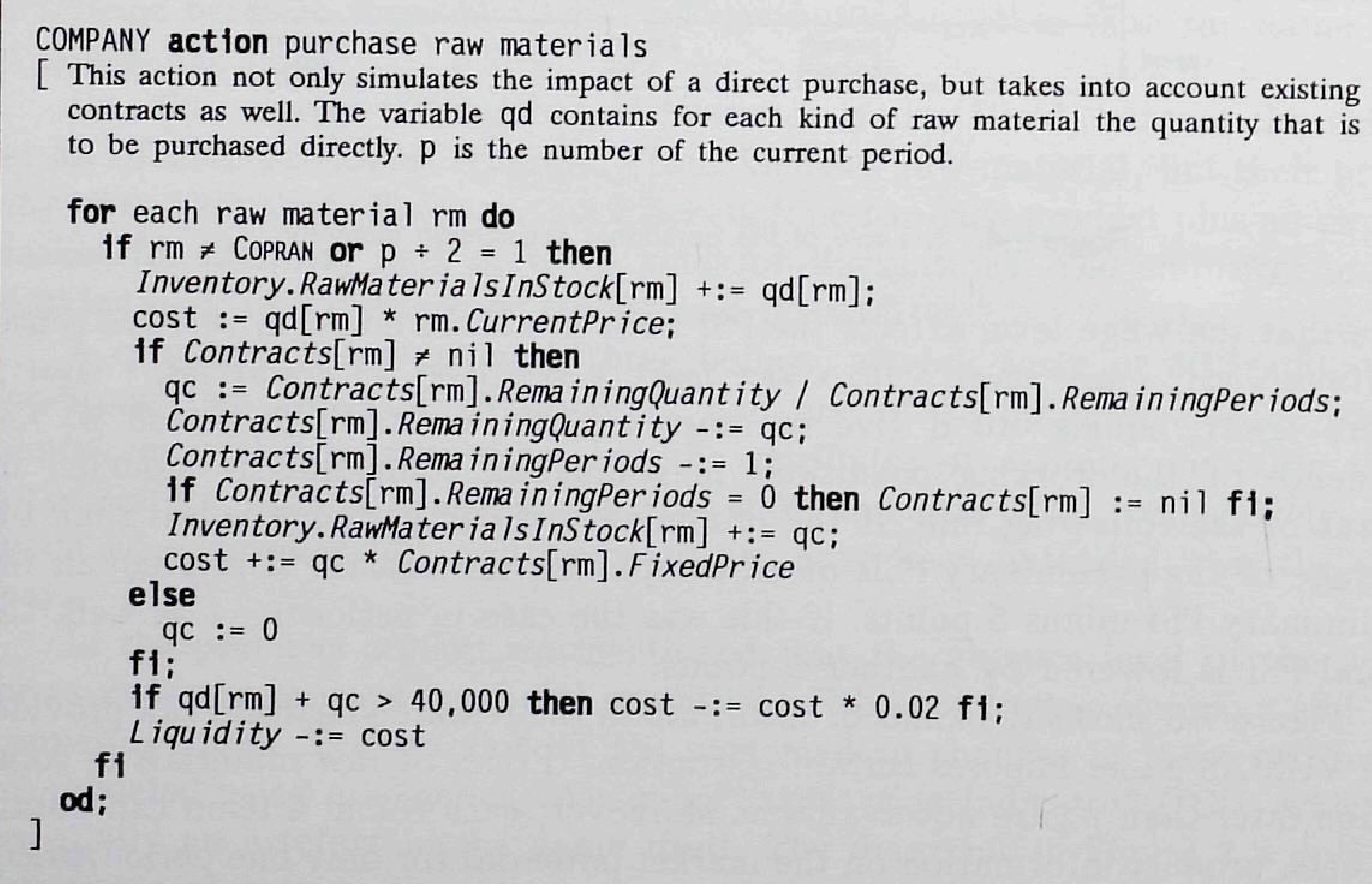

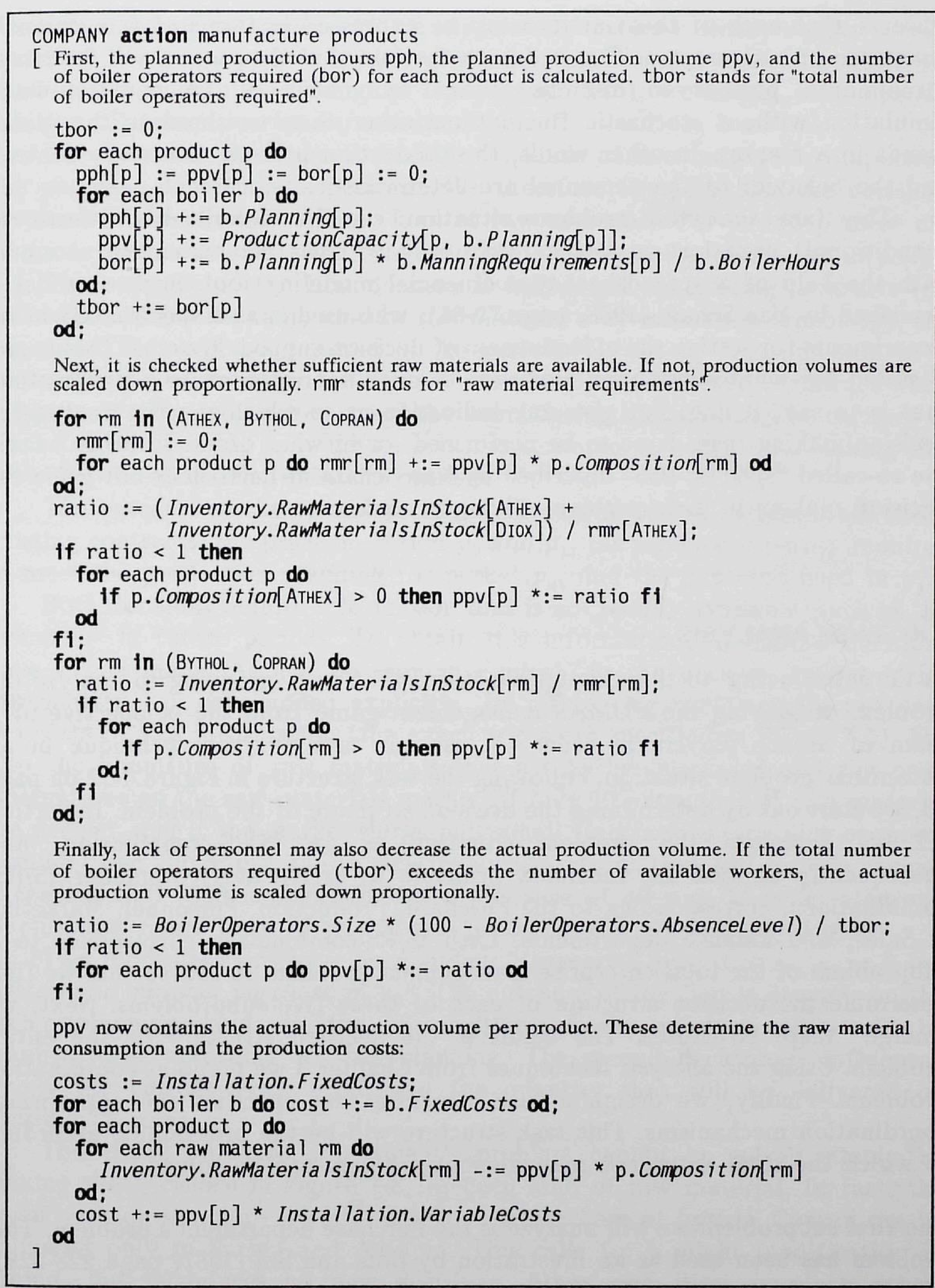

Flgure 7-6. The company action purchase raw materials

Figure 7-7. The company action manufacture products 
Observe that each of these actions can be expressed in terms of (conditional) equations or assignments. This is due to the fact that the game itself "reduces" three-month periods to discrete rounds by means of an equation-based simulation without stochastic fluctuations other than to simulate the global trends in a market. In other words, the production process, inventory control, and the behavior of the personnel are deterministic.

The fact that the problem situation can be described in terms of (conditional) equations makes it fairly easy to construct an empirical model with the help of a spreadsheet-type financial modeling tool. Such a model is described by Van Schaik (1988, page 79-85), who used it as a specific DSS in his experiments for testing the effectiveness of decision support systems. This model is suited for what-if analyses. However, it does not incorporate a programme, that is to say, it does not give any indication as to which specific analysis or decision-making tasks have to be performed, or in what order. In other words, the so-called "specific DSS" described by Van Schaik in itself does not guide the decision makers in their problem-solving process.

\subsection{TASK ANALYSIS}

In the introduction to this chapter we stated that we were going to analyze the problem of playing the VUMAS management game from the perspective of a team of novice players in order to test our task analysis technique in an unfamiliar problem situation. Following the task structure in Figure 4-12 on page 63 , we start out by determining the decision structure of the problem. In Section 7.2 we described the problem situation as an IS/RS-combination, and subsequently applied the recursion principle to arrive at five smaller IS/RScombinations, corresponding to the Purchase, Production, Personnel, Marketing \& Sales, and Finance departments. Each IS/RS-combination corresponds to a subproblem of the total enterprise management problem. In this section we first determine the decision structure of each of these five subproblems. Next, we "merge" these structures. The result is the decision structure of the entire problem. Using the analysis techniques from Chapter 3 we pinpoint coordination problems. Finally, we design a task structure that specifies the appropriate coordination mechanisms. This task structure will be the programme according to which the total enterprise management problem can be solved.

The first subproblem we will analyze is the Purchase department's problem. This problem has been used as an illustration by Bots and Sol (1987, page 228-229). Looking at the list of decisions the players have to make each round, we see two decisions that obviously have to be made by this department: "Purchase quantities of four kinds of raw materials directly from the suppliers" and
"Initiate one-year contracts with the suppliers to ensure a steady supply of raw materials at a fixed price". In view of the information that can be bought from the research institute (see Figure 7-5 on page 186), we might also add the decision "Purchase market information".

The fact that there are four different kinds of raw materials complicates the purchase department's problem. We therefore first consider the decisions involved in the purchase of one kind of raw materials. Per period $p$ these are "Initiate new contract?" and "Determine the quantity to be purchased directly".

The outcome of the first decision is influenced by existing contracts (no two contracts for the same kind of raw material may exist simultaneously), the quantities that will be required in the periods $p_{1}, \ldots, p_{4}$ (the duration of a contract), the expected prices in those periods (do not initiate a contract when the price is expected to drop), and the warehousing costs (it may be cheaper to stock for one period, as is the case in the example on page 211). Making this decision will affect the existing contracts.

The outcome of the second decision will depend on the current stock level, existing contracts, the prices in period $p_{0}$ and $p_{1}$, the minimal quantity required to meet the production planning for period $p_{0}$, and the expected need in $p_{1}$.

Both decisions require information that is not readily available, such as the quantities in future periods. To obtain this information, additional decisions have to be made. As can be seen in Figure $7-8$, the decision to buy price information has been added explicitly. Its influencing part includes not only the costs of this information (the exact prices are specified in Figure 7-5), but also the quantities of raw materials that are to be purchased and the price fluctuations on the raw materials market. If only 10 extra tons of raw material are needed, and if the market shows only small fluctuations (say plus or minus 1 guilder per kilogram), the chance that price information will lead to savings exceeding NLG 7500 is slight. But if a purchase of 40 tons is anticipated and fluctuations range from -5 to +5 , this probability is substantially higher.

Two more decisions have been added: "Determine quantities $q_{0}, \ldots, q_{4}$ " and "Determine minimal purchase in $p_{0}$ ". The outcome of the first is depends on the boiler planning, and on the composition of the products that will be manufactured according to that planning. The second decision is influenced by $q_{0}$, the current stock level, and the quantity that will be delivered on contract (if any).

The entire Purchase department's problem cannot be solved simply by making the decisions in Figure 7-8 for each kind of raw material. In fact, the given decision structure is valid only for the purchase of Bythol. Copran can be delivered only in Spring and Autumn. This calls for a slight modification in timing, but introduces no new decisions. Athex and Diox are each others substitute, which means that the stock level, contract and price for two kinds of raw materials must be checked instead of one. Again, no new decisions are 
required, but making the existing ones will be more complicated. We shall return to this when designing a task structure for the problem.

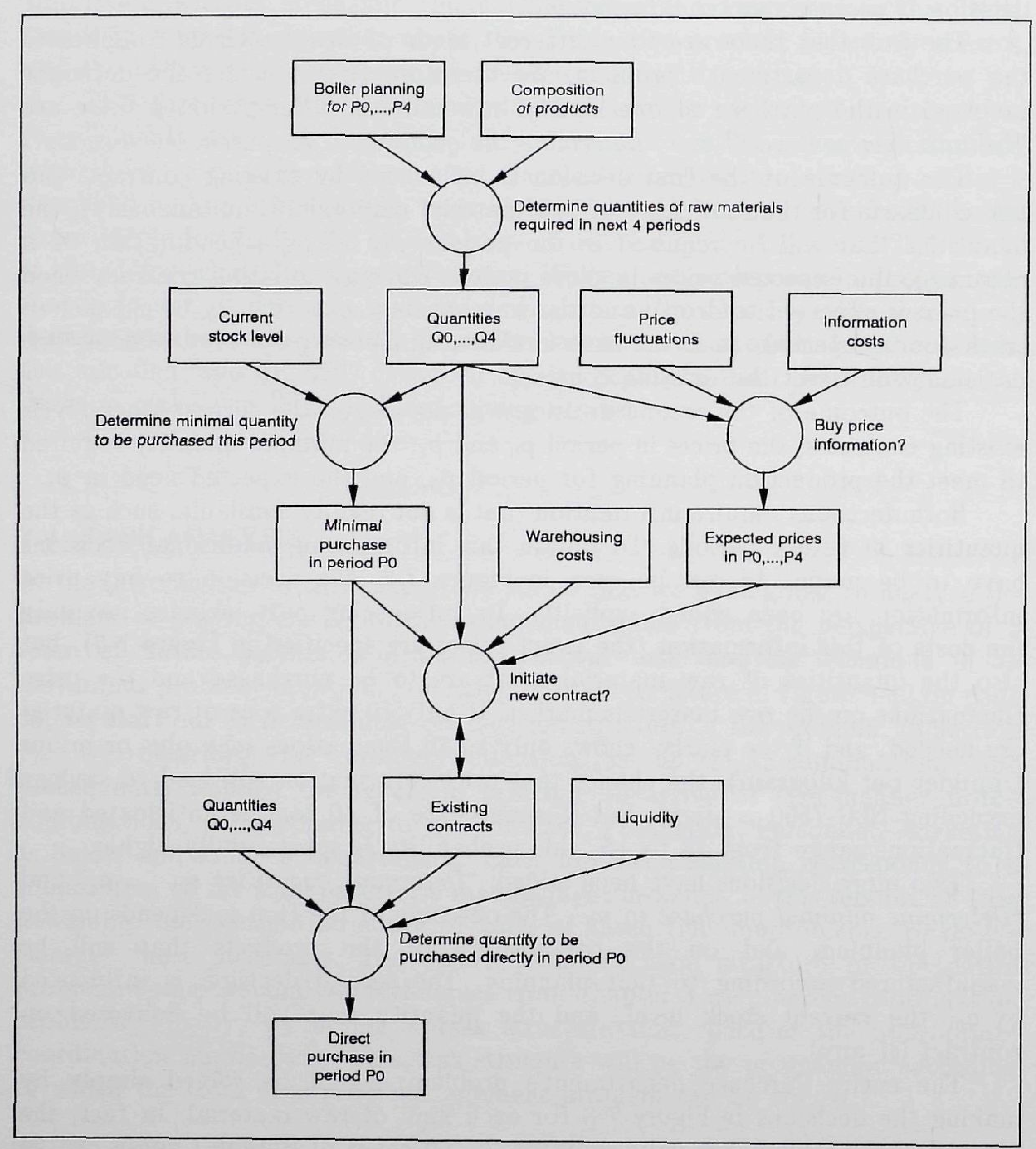

Flgure 7-8. The decision structure of "Purchase one kind of raw materials"

Provided that all the influencing information of the decisions in Figure 7-6 is available or generated by some other decision in that decision structure, optimization is possible. Note that, with the exception of the prices, all information items are either invariant (the composition of products, warehousing costs) or under control of the company. Information on the prices of raw materials can be obtained from the research institute. We shall present a number of rules for the decisions when discussing the support component of the purchase task in Section 7.4.

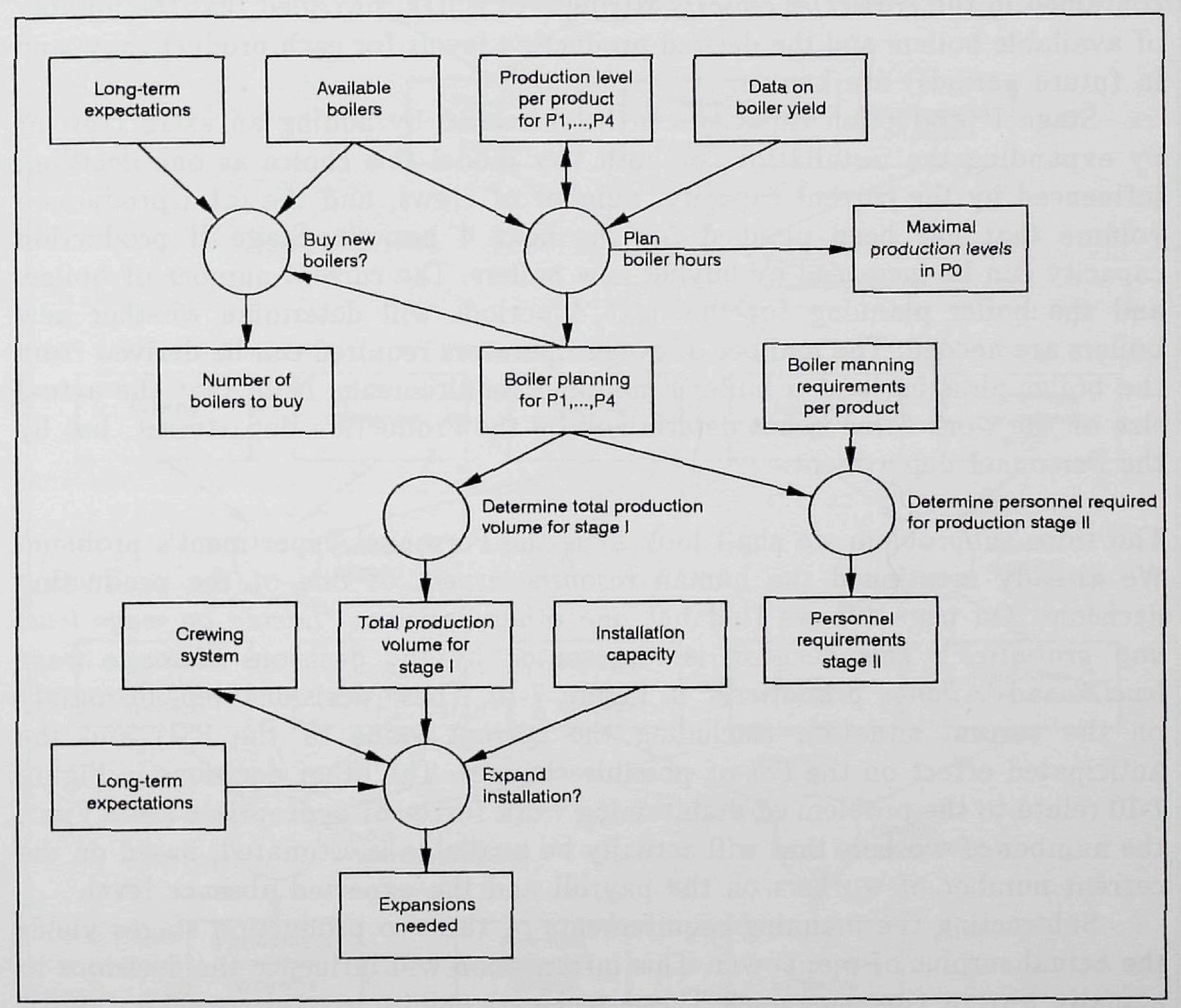

Figure 7-9. The decision structure of the Production department's problem

The next subproblem we consider is the Production department's problem. Again, we consult the list of game decisions on page 171, where we find two decisions that are directly related to production: "For two production stages, expand or reduce the production facilities and determine the size of the work force" and

"For each of the five products, set production levels for the next quarter". The 
first of these will depend on the outcome of the second. Both decisions can be split into smaller ones, as is shown in Figure 7-9.

The game requires production to be planned one period in advance, meaning that in period $p_{0}$ one has to plan the boiler hours for period $p_{1}$. But in view of the information required by the purchase department it makes sense to plan four periods ahead, even though the figures for later periods may still be subject to change. Boiler hours can be planned using the data on boiler yield contained in the Production capacity attribute of BOILER, provided that the number of available boilers and the desired production levels for each product (now and in future periods) are known.

Stage I production capacity can be increased by adding an extra crew or by expanding the installation, or both. We model this choice as one decision, influenced by the current capacity, number of crews, and the total production volume that has been planned for the next 4 periods. Stage II production capacity can be increased by buying new boilers. The current number of boilers and the boiler planning for the next 4 periods will determine whether new boilers are needed. The number of boiler operators required can be derived from the boiler planning and a boiler's manning requirements. Note that the actual size of the work force is not determined by the Production department, but by the Personnel department.

The third subproblem we shall look at is the Personnel department's problem. We already mentioned the human resource aspect of one of the production decisions. On page 171 we find but one other decision: "Decide on wage level and gratuities". This decision is represented by the decisions "Change wage level?" and "Change gratuities?" in Figure 7-10. These decisions depend mainly on the current situation (including the current value of the PSI) and the anticipated effect on the PSI of possible changes. The other decisions in Figure 7-10 relate to the problem of maintaining work forces of appropriate sizes. First, the number of workers that will actually be available is estimated, based on the current number of workers on the payroll and the expected absence level.

Subtracting the manning requirements of the two production stages yields the actual surplus of manpower. This information will influence the decisions to actually hire or fire personnel. These decisions require careful consideration of the long term expectations with respect to the company's investments and future production levels. Since the consequences of lay-off are quite severe, the costs and benefits of firing a substantial group of workers must be weighed carefully. Since lay-off of more than five workers in one period also affects the PSI, the risk of a strike must be considered as well.

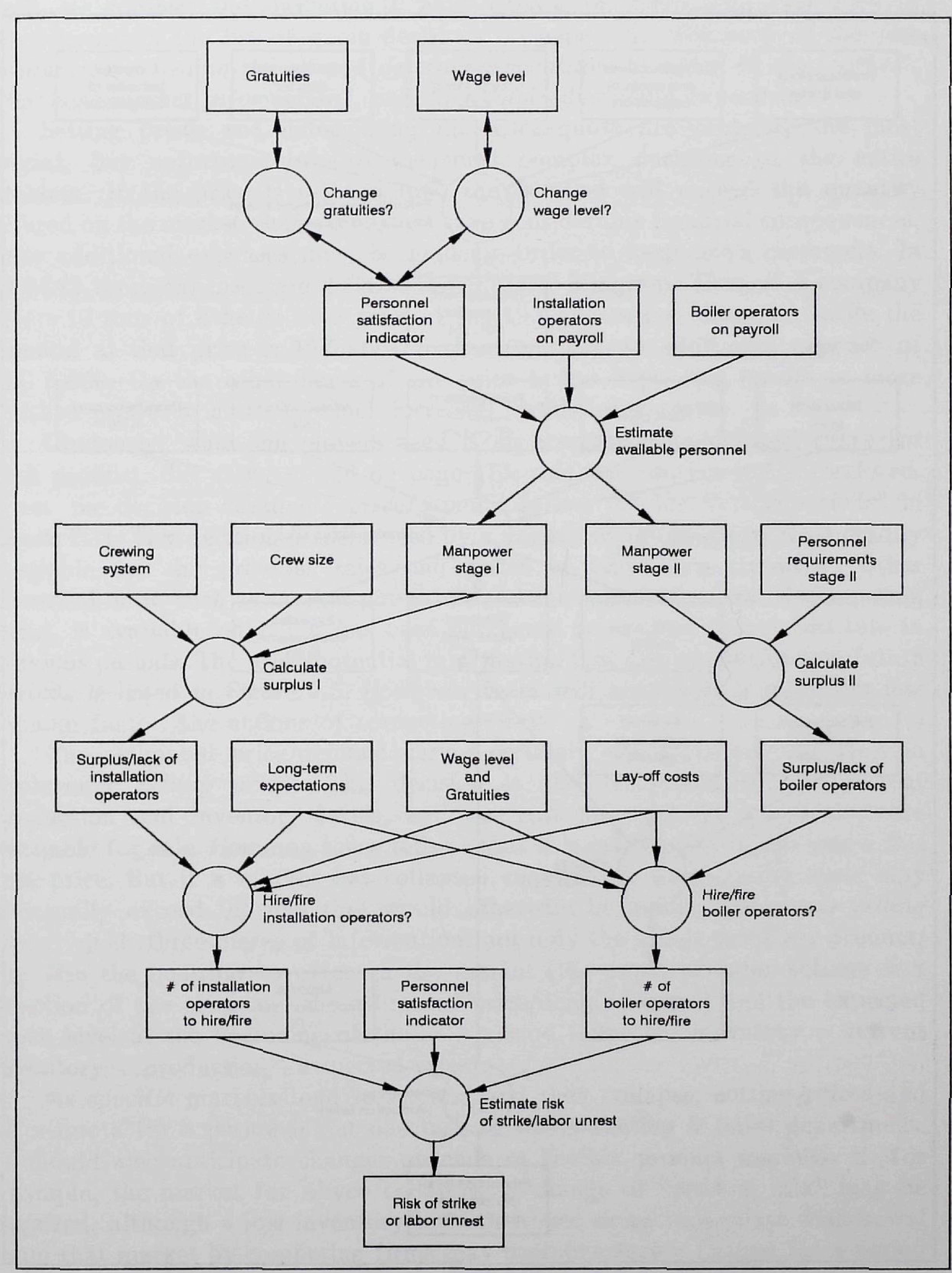

Fgure 7-10. The decision structure of the Personnel department's problem 


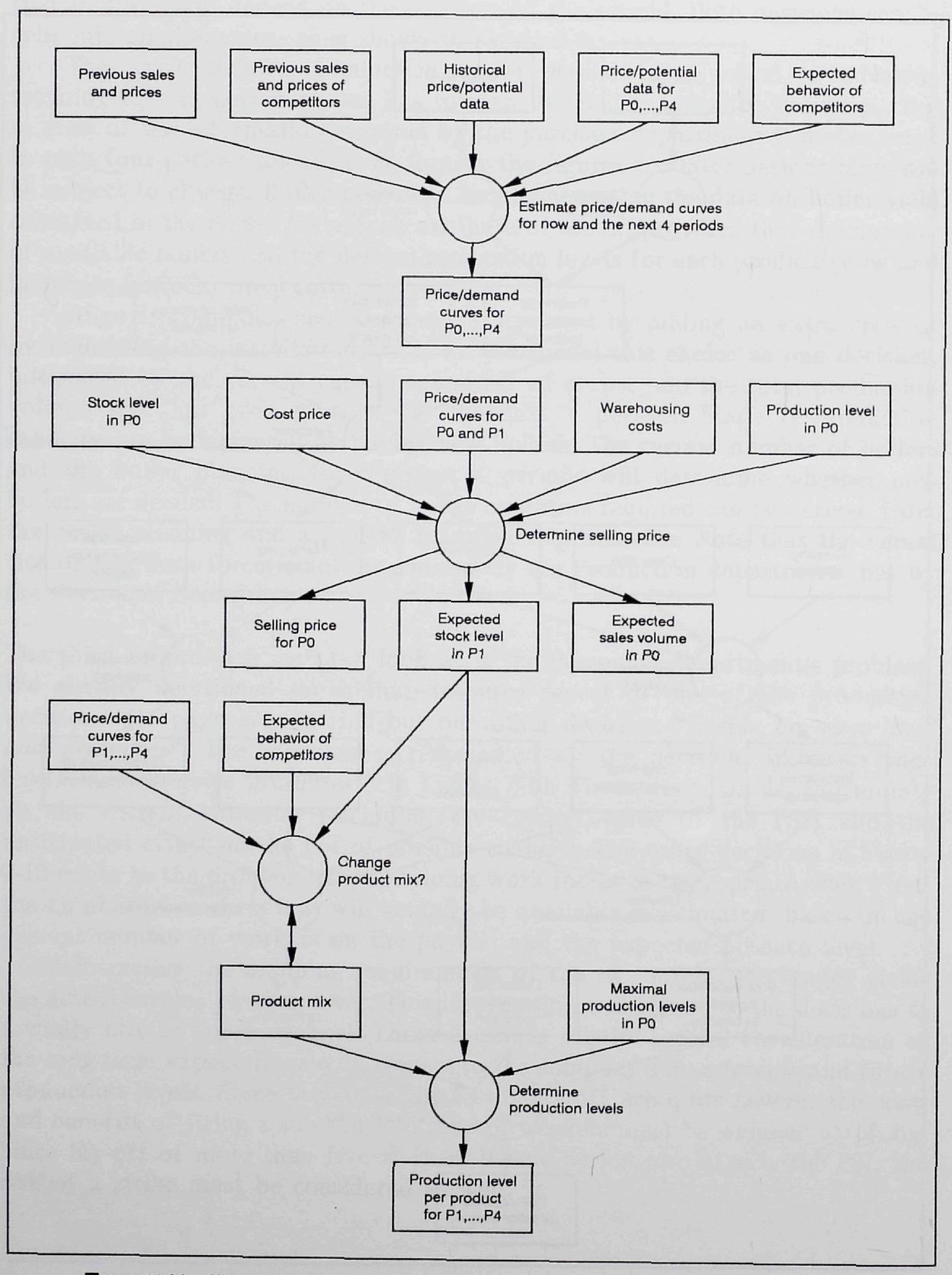

Figure 7-11. The decision structure of the Marketing \& Sales department's problem
Next, we consider the Marketing \& Sales department's problem. This involves three items on the list of game decisions on page 171: "For each of the five products, set selling prices and determine quantities to offer on the market", "Purchase market information", and "Set total advertising expenditure".

Setting prices and determining the sales quota are probably the most crucial, but unfortunately also the most complex decisions of the entire problem. If the price is set too low, the demand will exceed the quantity offered on the market. Such stock-outs have considerable financial consequences, since additional expenses must be made in order to keep one's customers. In VUMAS these expenses are 2 Guilders per "lost" kilogram. Thus, if a company offers 10 tons of Ethelite at a price of say 19 Guilders per kilogram, while the demand at that price is 12.5 tons, it has to make an additional expense of NLG 5,000. On the other hand, if the price is too high, this results in more finished products in stock, which increases warehousing costs.

Obviously, what the players need is an accurate price/demand curve for each product. But as we stated on page 173 , this information is not disclosed. Hence the decision "Estimate price/demand curves for the next 4 periods" in Figure 7-11. This decision is influenced by a variety of information. Most readily available are the previous sales and prices of one's own company. Other historical data, such as market potentials and the sales and prices of competing firms, is available only if it has been purchased from the research institute in previous periods. The price/potential information that can be obtained on future periods is listed in Figure 7-5. However, there will always be a more ore less random factor: the actions of competing firms.

The estimated price/demand curves certainly will influence the decision "Determine selling price". This decision is also influenced by the current production and inventory levels, and the cost price of the goods that are available for sale. Common sense tells us that it is inadvisable to sell below this cost price. But if a market has collapsed completely, warehousing costs may eventually exceed the loss that would otherwise be made. "Determine selling price" yields three pieces of information: not only the selling price per product, but also the quantity to offer on the market (the expected sales volume is a function of the price and the estimated price/demand curve) and the expected stock level at the beginning of the next period (expected inventory = current inventory + production - expected sales).

As specific markets tend to expand and then collapse, setting prices and sales quota for a period is but one task of the Marketing \& Sales department. It should also anticipate changes on each of the six product markets. If, for example, the market for Abyco collapses, a change of "product mix" may be required, although a low inventory level of Abyco or an immediate withdrawal from that market by competing firms may postpone such a change for a period 
or two. The product mix eventually determines the production planning for future periods.

So far, we have covered two of the three items selected earlier from the list of game decisions on page 171. We now consider the third one: "Set total advertising expenditure". The VUMAS game allows advertising for Abyco only. We therefore add a single decision to the decision structure of the Marketing \& Sales department: "Determine advertising budget". This decision is influenced by the current product mix (it is of no use to advertise if one does not sell Abyco), the Abyco price/demand curves, the estimated effect of advertising, and the anticipated advertising actions of competitors. The fact that advertising has an impact on the price/demand curves is represented in Figure 7-12 by the doubleheaded arrow.

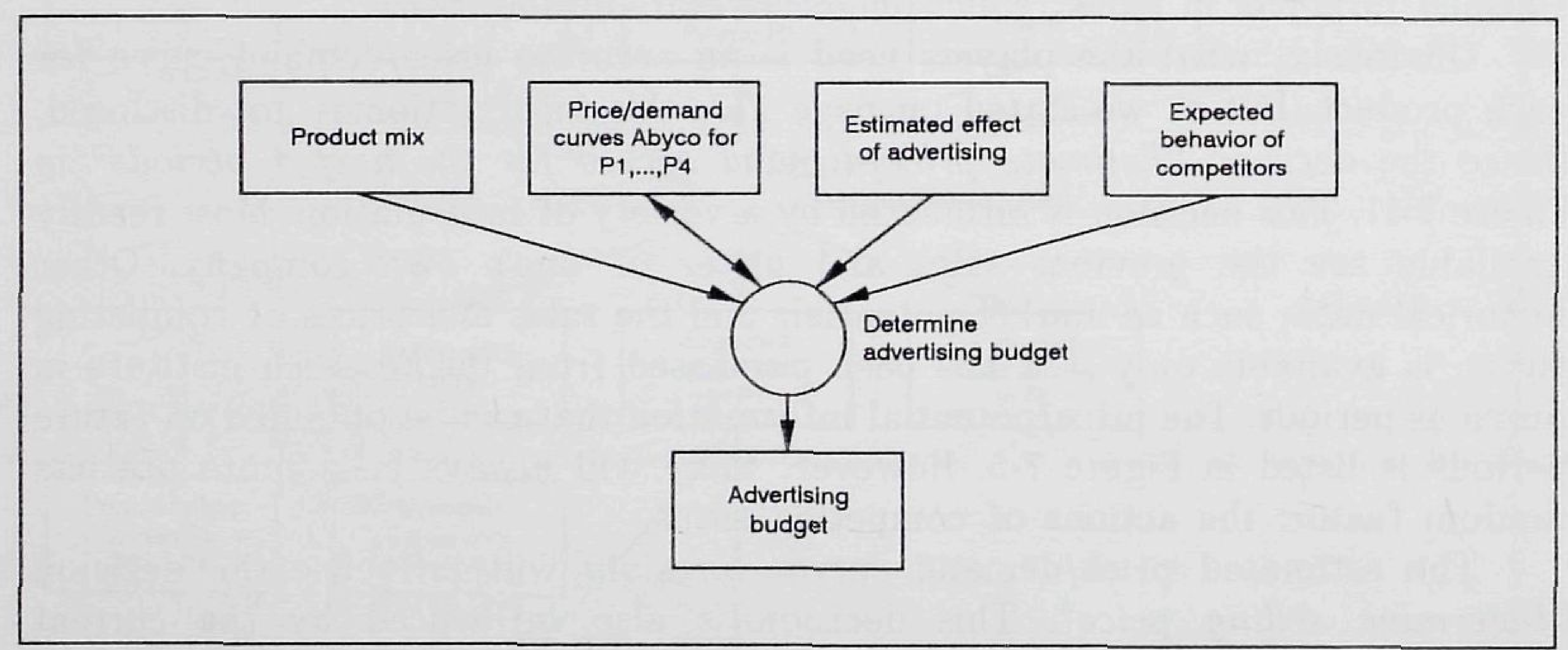

Flgure 7-12. The decision structure of the Marketing \& Sales department's problem (continued)

The last subproblem we investigate is the Finance department's problem. This problem relates to the last three items of the list on page 171: "Borrow shortterm or long-term funds", "Pledge for government support", and "Determine dividend to be paid to shareholders". In other words, the Finance department is responsible for maintaining good relationships with the bank, the government, and the share holders.

The bank offers two lines of credit: short-term credit to cover temporary liquidity shortages (short-term funds have to be paid back within three periods), and long-term credit to finance investments in installation capacity or new boilers. We therefore identify two decisions for obtaining loans. "Ask for shortterm loan?" mainly depends on the company's liquidity in period $p_{1}$, which in turn depends on the liquidity in $p_{0}$, and the expected sales and estimated total costs in $p_{1}$. The decision "Ask for (new) long-term loan?" depends on the current liquidity situation, the size of the planned investments, and on the amount of credit already offered by the bank. Obviously, the bank's interest rates of some $6 \%$ (short-term) and 21/2-3\% (long-term) per period are important factors as well.

If the bank refuses to give any more credit - usually because it has granted too many loans already, hence the influencing part of "Pledge for support?"the company will have to turn to the government for financial support.

Dividend may be declared in Spring and Fall periods. The dividend percentage will largely depend on the company's net profit after tax. In addition to "Declare dividend?" we therefore identified the decision "Determine taxes $d u e^{\prime \prime}$.

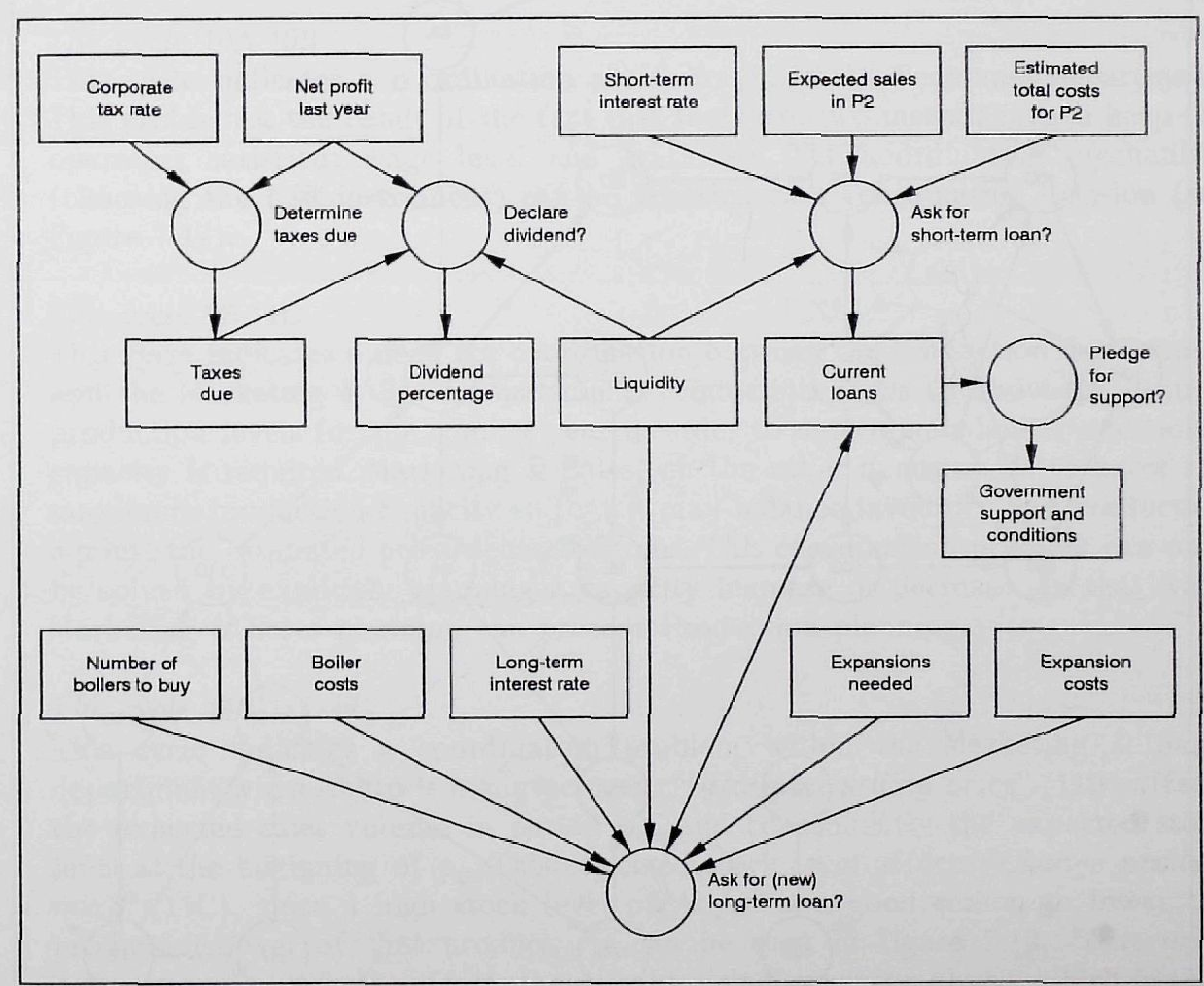

Fgure 7-13. The decision structure of the Finance department's problem 
Having determined the decision structure of each of the five subproblems identified in Section 7.2, we can construct the decision structure of the task of playing a round of the VUMAS game by merging the decision structures of Figure 7-8 through 7-13. The result is shown in condensed form in Figure 7-14

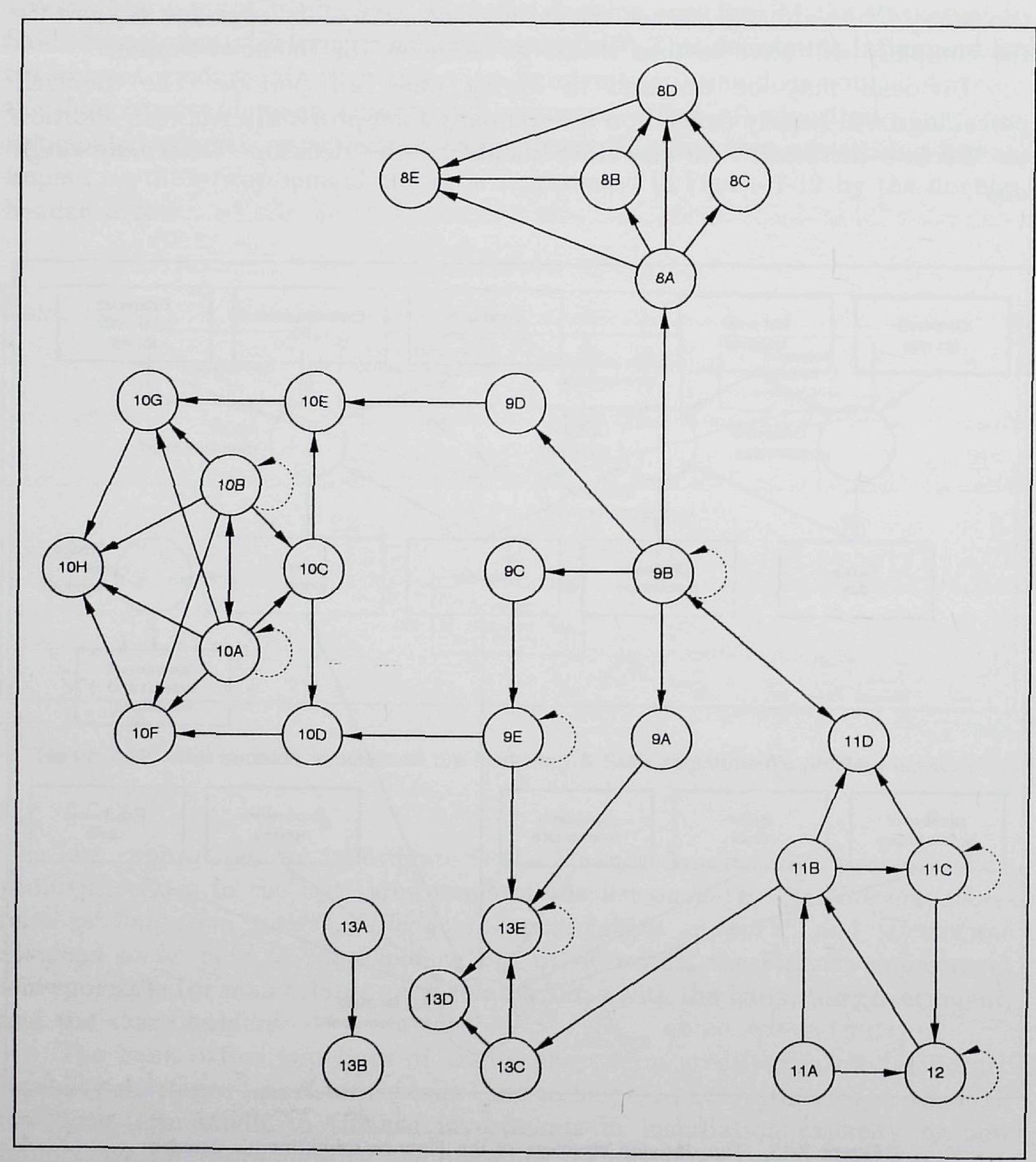

Figure 7-14. The condensed decision structure of the total enterprise management problem
Like we did in the preceding chapters, the decisions have been labeled with a code instead of their actual name for reasons of readability. The numbers correspond to those of the figures in this chapter. Decisions from figures containing more than one decision are enumerated A, B, C, etcetera, first from left to right, then from top to bottom. Thus, "Buy price information?" and "Initiate new contract?" in figure $7-8$ have codes $8 \mathrm{C}$ and $8 \mathrm{D}$, respectively, while "Determine advertising budget" in Figure 7-12 has code 12.

The condensed decision structure reveals a number of dependencies between the five subproblems. It also contains three cycles involving more than one decision. Each of these dependencies and cycles may indicate a coordination problem. We will examine them systematically, determine whether coordination is required, and if so, what kind of mechanism should be used.

The cycle 10A-10B

This cycle indicates a coordination problem within the Personnel department. This problem is the result of the fact that there are two instruments to keep the operators satisfied: wage level and gratuities. The coordinating mechanism (choosing the best instrument) can be modeled as a coordinating decision (see Figure 7-17).

\section{The cycle 9B-11D}

This cycle indicates a need for coordination between the Production department and the Marketing \& Sales department. Production needs to know the desired production levels for the coming year in order to determine whether additional capacity is required. Marketing \& Sales, on the other hand, needs to know the maximum production capacity so that it may balance inventory and production against the estimated price/demand curves. This coordination problem can only be solved by explicitly planning a capacity increase or decrease. In that way, Marketing \& Sales planning can precede Production planning.

\section{The cycle 11B-11C-12}

This cycle indicates a coordination problem within the Marketing \& Sales department when Abyco is manufactured. "Determine selling price" (11B) affects the expected sales volume in period $p_{0}$, and consequently the expected stock level at the beginning of $p_{1}$. This expected stock level affects "Change product mix?" (11C), since a high stock level of Abyco is a good reason to lower the production level of that product. As can be seen in figure 7-12, "Determine advertising budget" (12) affects the price/demand curve for Abyco, which in turn influences "Determine selling price" again. This causes the cycle 11B-11C-12. This cycle can be broken by first deciding whether the production of Abyco should be continued or stopped. This decision "cuts" the edge $11 \mathrm{C}-12$, and the natural 
sequence of decisions becomes 12-11B-11C. This sequence may of course be "executed" several times in order to find a profitable product mix.

The bridge 9B- $8 \mathrm{~A}$

This edge indicates the implicit coordinating mechanism between the Production department and the Purchase department: Once Production has determined the actual production volumes, Purchase can perform its task without needing any additional information from other departments. In other words, "Purchase raw materials" is an independent task (see page 43). Consequently, we can at this point design a task structure for this task.

Recall that the decision structure in Figure 7-8 relates to the purchase of one kind of raw material only. This also holds for the task structure in Figure $7-15$, which is generic in the sense that it specifies a programme for the purchase of any of the four kinds of raw material. The rules for the coordinating decisions will be given in the next section.

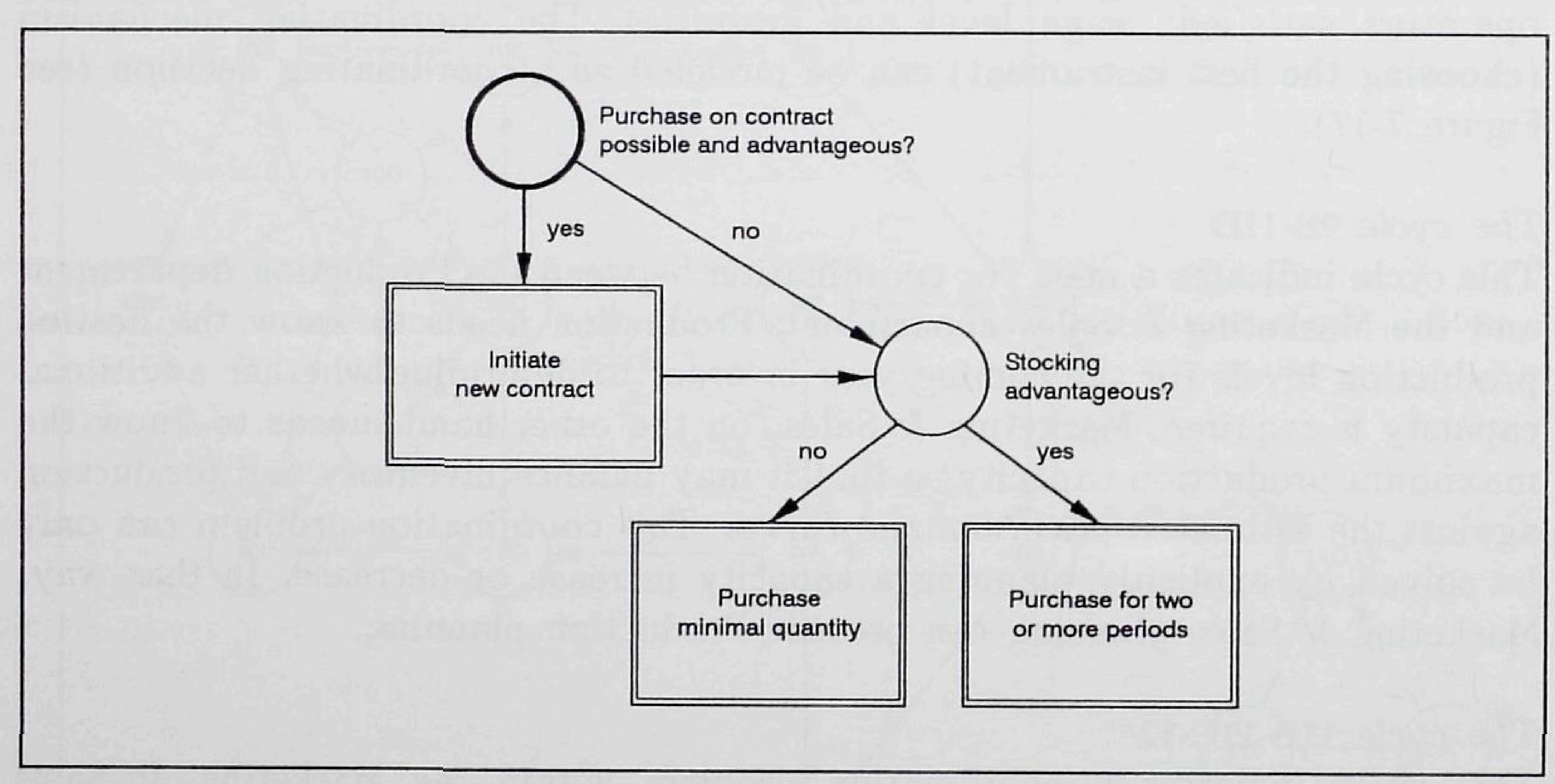

Fgure 7-15. A task structure for "Purchase one kind of raw material"

Earlier we remarked that the fact that Athex and Diox are each other's substitute, and that Copran is available only in Spring and Fall periods would cause coordination problems. The task structure in Figure 7-16 shows how these problems can be solved by means of coordinating decisions. Note that the decision "Price of Athex in $p_{0}$ less than price of Diox?" is a criterion for both contractual and direct purchase.

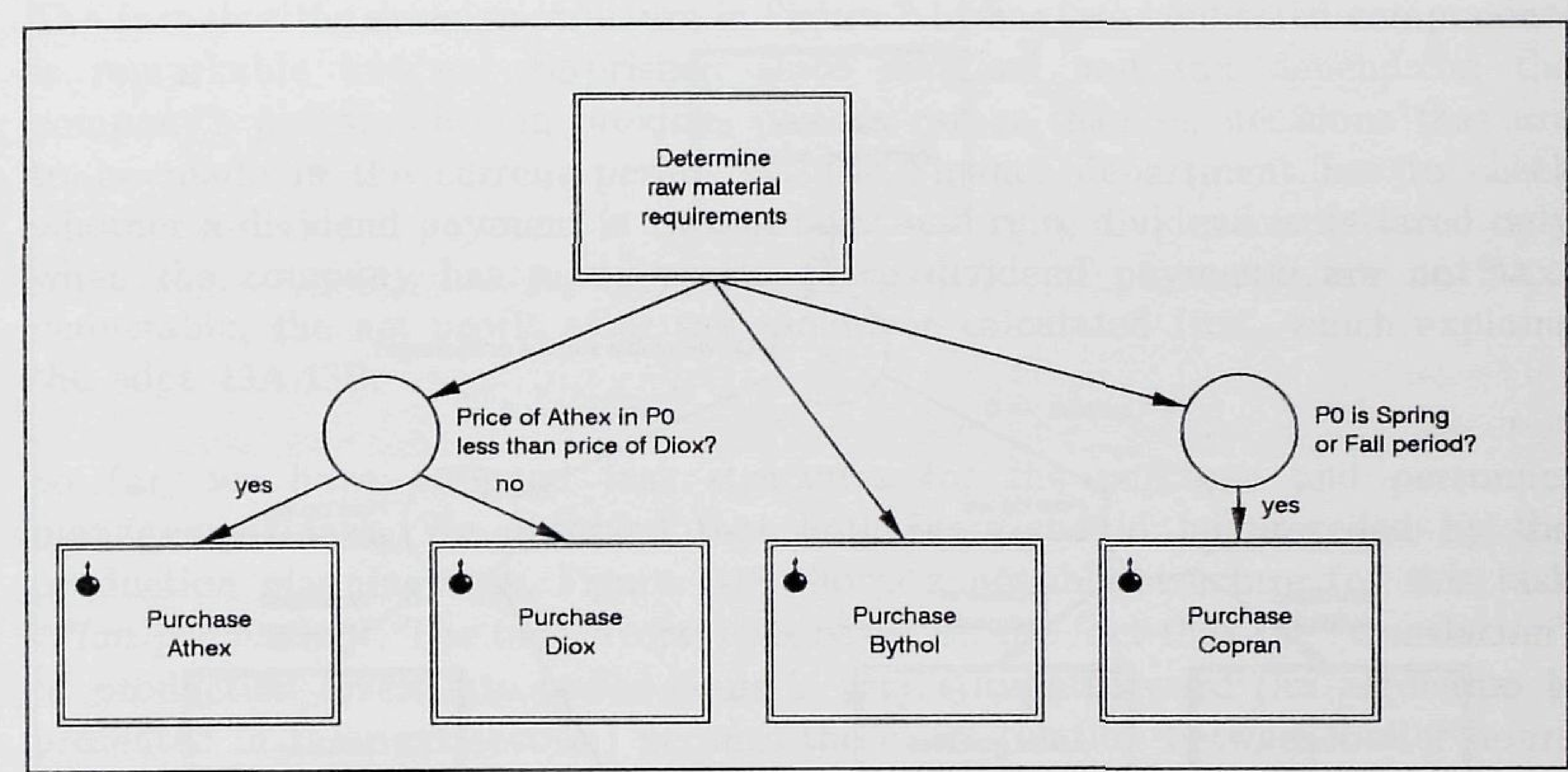

Flgure 7-16. A task structure for "Purchase raw materials"

The edges 9D-10E and 9E-10D

These edges also indicate implicit lead-follow coordination, this time between the Production department and the Personnel department: Production passes its manning requirements for both production stages on to Personnel, which can subsequently perform its task without further inter-departmental coordination. Figure 7-17 shows a possible structure for the Personnel department's task. Decisions $10 \mathrm{D}$ and $10 \mathrm{E}$ are made during "Determine surplus". If there is substantial surplus or shortage (indicated by the labels "surplus $\gg 0$ " and "surplus « 0", respectively), the appropriate instrument(s) for correcting this deviation must be chosen. This choice is made explicit by means of the first three coordinating decisions. We speak of an "incidental" personnel surplus or shortage if it is expected to last for one or two periods, and "structural" otherwise.

The solution for a structural shortage is simple: hire extra personnel. In case of a structural surplus, firing personnel is necessary to avoid redundancy costs. When the surplus is incidental, these costs can be reduced by temporarily decreasing the wage level or by cutting on gratuities. Since these are rather harsh measures, one should consider the risk of a strike. If this risk is judged too high, one might look for ways to create work, for instance by producing more labor-intensive products. Producing less labor-intensive products may solve an incidental lack of personnel. Since such decisions are beyond the scope of the Personnel department, they are signalled to Marketing \& Sales. 


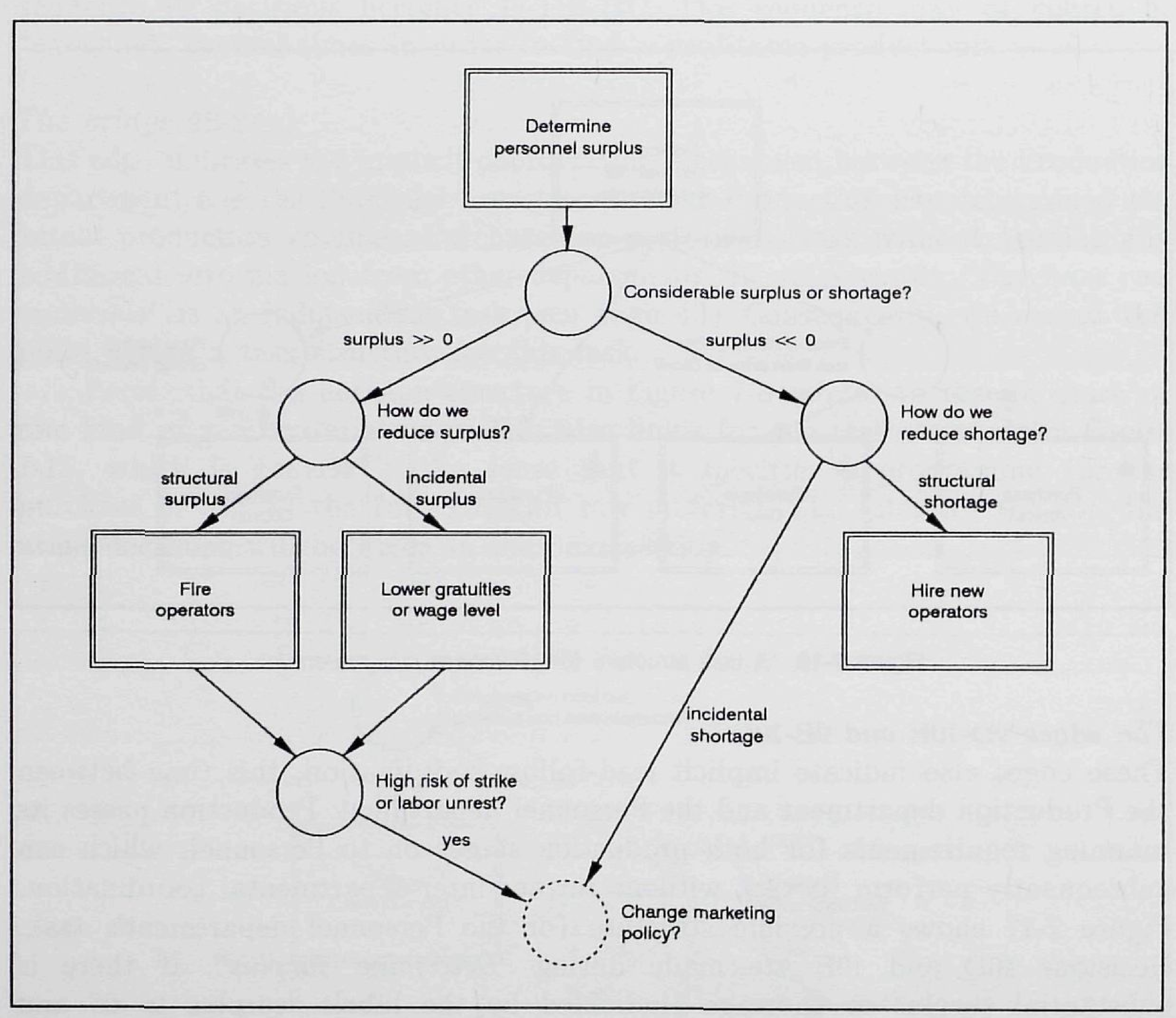

Fgure 7-17. A task structure for "Manage personnel"

Note that detecting this particular inter-departmental coordination problem is a matter of common sense: It could not be derived directly from the decision structure in Figure 7-14. One has to see the logical consequences of decision $10 \mathrm{H}$, and subsequently check with the conceptual model in order to determine for whom these consequences are important.

The edges 9A-13E, 9E-13E, and 11B-13C

These edges show that there is no straightforward lead-follow coordination between the Production department and the Finance department. The long-term loan decision $13 \mathrm{E}$ has to be made only if Production has insufficient capacity. The need for short-term loans depends on the sales and total costs as expected in the next period.
The connected component $\{13 \mathrm{~A}, 13 \mathrm{~B}\}$

The fact that the decision structure in Figure 7-14 has two connected components is remarkable but not surprising, since dividend and tax depend on the company's performance in previous periods rather than on decisions that are to be made in the current period $p_{0}$. The Finance department has to check whether a dividend payment is appropriate. As a rule, dividend is declared only when the company has made profit. Since dividend payments are not taxdeductable, the net profit after tax should be calculated first, which explains the edge 13A-13B.

So far, we have designed task structures for the purchase and personnel management task. We observed that both tasks should be preceded by the production planning task. Figure 7-18 shows a possible structure for this task "Plan production". The task structure is based on the fact that the "translation" of production levels into boiler hours is very straightforward (an algorithm is presented in the next section) because the exact relation between boiler hours and production volume is known. This translation includes determining the number of operators required for both production stages, the installation capacity for stage I, and the number of boilers for stage II. Thus, decisions 9A through 9E are made during "Plan production". The fact that the Production department cannot make investment decisions alone requires a coordinating mechanism between Production and Finance. This mechanism is visible as two signals at the bottom of Figure 7-18.

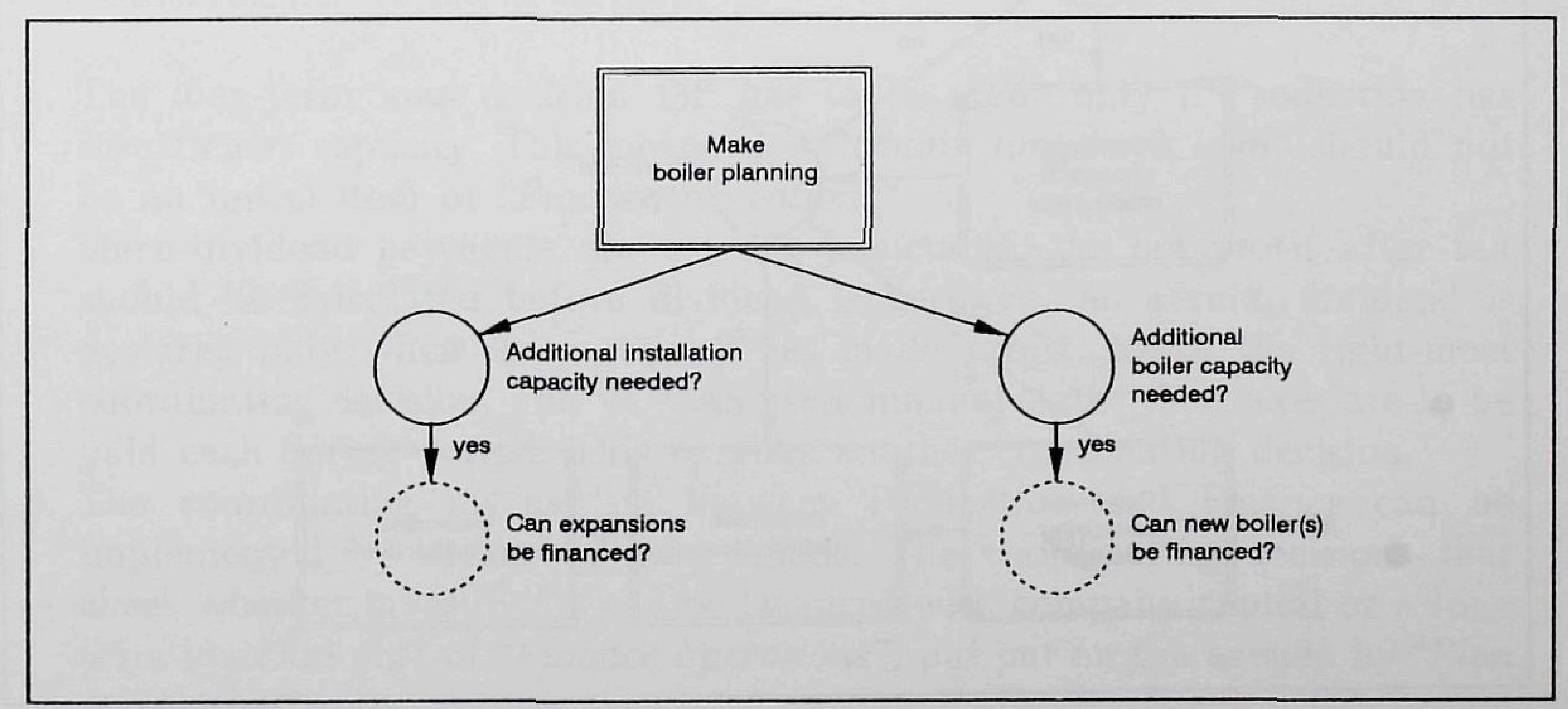

Figure 7-18. A task structure for "Plan production" 
The decision structure in Figure 7-13 indicates that the first task of Marketing \& Sales is to estimate price/demand curves for each product market. This activity is followed by the cycle $11 \mathrm{~B}-11 \mathrm{C}-12$ in Figure $7-14$. Earlier we pointed out that this cycle can be broken by first deciding whether the production of Abyco should be continued or stopped. This decision corresponds to the coordinating decision "Do we sell Abyco?" in Figure 7-19. The order in which the decisions 11B, 11C and 12 should be made has been fixed by "sequencing" the corresponding subtasks "Determine advertising budget", "Determine selling prices", and "Determine production levels" in the flow relation (see page 48). The subtask "Consider change of product mix" is put on the agenda only if one or more product markets are expected to grow or collapse.

Note that the task of making long-term forecasts cannot be traced back directly to one of the decisions in Figure 7-11 or any one of the other decision structures: It is the information item "Long-term expectations" in Figure 7-9 that indicates the need for this task. For this reason, we decided to add "Make longterm forecast" as a subtask of "Market products".

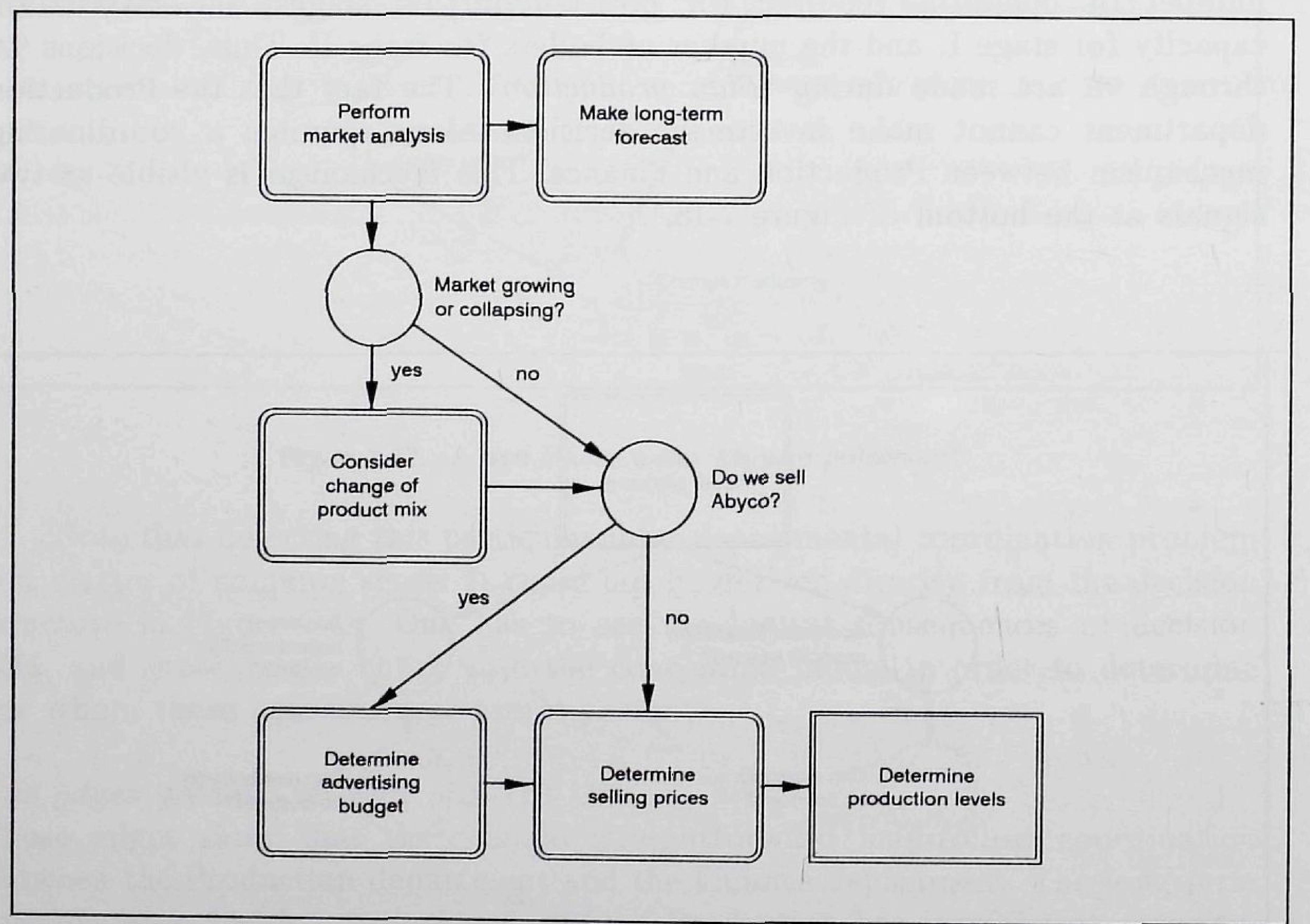

Fgure 7-19. A task structure for "Market products"

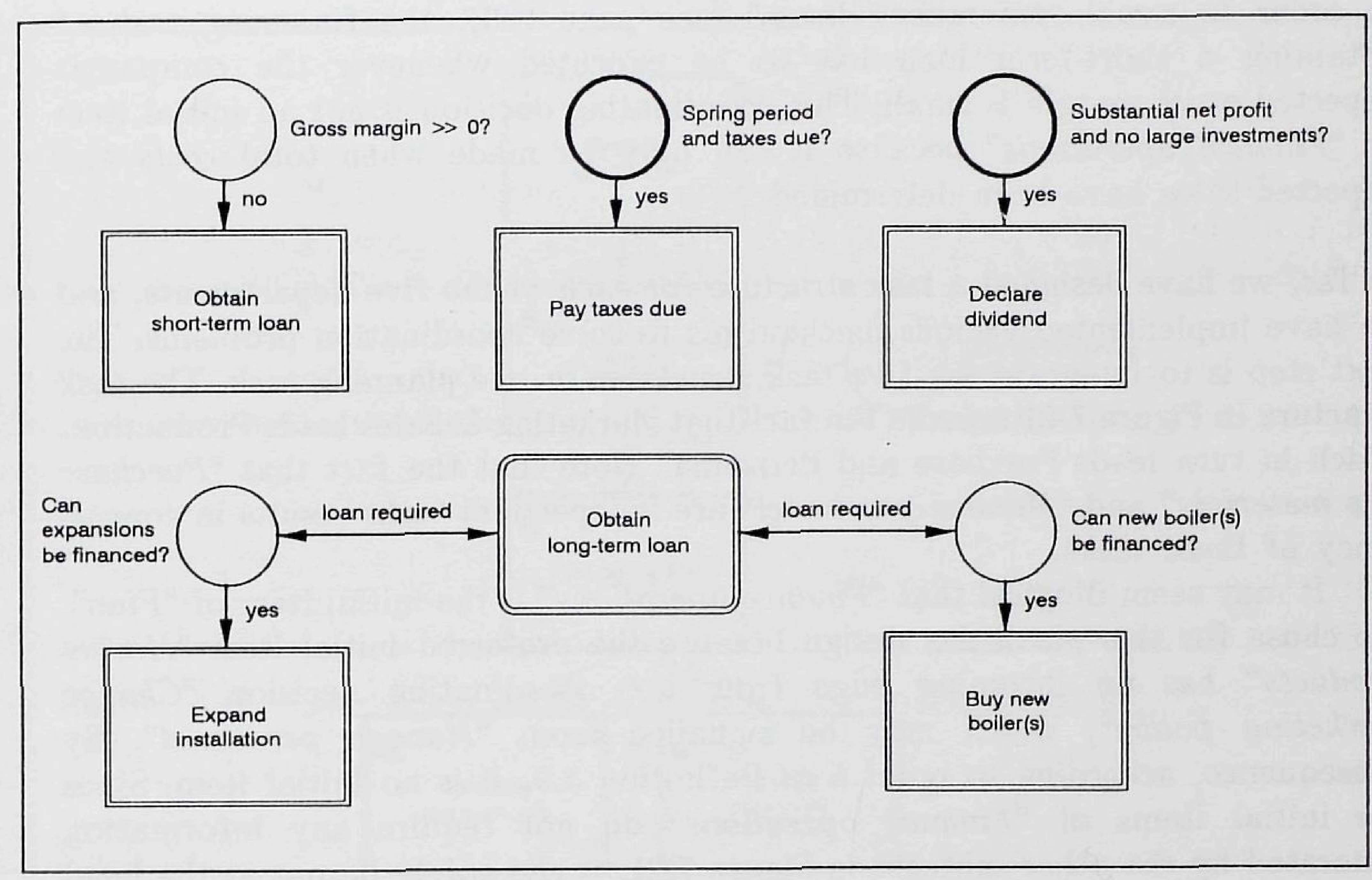

Figure 7-20. A task structure for "Finance operations"

Figure 7-20 depicts a possible task structure for the task of financing a company's operations. The design of this particular task structure is based on three observations we made earlier:

1. The long-term loan decision $13 \mathrm{E}$ has to be made only if Production has insufficient capacity. This means that "Obtain long-term loan" should not be an initial item of "Finance operations".

2. Since dividend payments are not tax-deductable, the net profit after tax should be calculated before dividend is declared. As a rule, dividend is declared only when the company has made profit, hence the right-most coordinating decision. The VUMAS users manual states that taxes are to be paid each Spring period. This requires another coordinating decision.

3. The coordinating mechanism between Production and Finance can be implemented by means of two signals: The coordinating decisions that check whether investments can be financed with company capital or a long term loan are part of "Finance operations", put put on the agenda by "Plan production" only. 
In order to avoid "emergency loans" (see page 180), the financing task of obtaining a short-term loan has to be executed whenever the company's expected gross margin is small. This coordinating decision is not an initial item of "Finance operations" because it can only be made when total costs and expected sales have been determined.

So far, we have designed a task structure for each of the five departments, and we have implemented various mechanisms to solve coordination problems. The next step is to integrate the five task structures in one planning task. The task structure in Figure 7-21 mirrors the fact that Marketing \& Sales leads Production, which in turn leads Purchase and Personnel. Note that the fact that "Purchase raw materials" and "Manage personnel" are independent tasks results in concurrency of these tasks.

It may seem illogical that "Finance operations" is the initial item of "Plan". We chose for this particular design because the preferred initial item "Market products" has an incoming edge from the coordinating decision "Change marketing policy", which may be signalled from "Manage personnel". By consequence, according to point 5 of Definition 3.9 , it is no initial item. Since the initial items of "Finance operations" do not require any information generated by the other subtasks in Figure 7-21, it can safely be put at the head of the sequence. An alternative solution would have been to design "Plan" in such a way that it has no initial items at all, in which case "Market products" can be chosen each time "Plan" is initiated (see page 71). The flow relation of "Plan" should in this case include an edge from some subtask to "Finance operations". We find this solution even less elegant than the one depicted below.

Obviously, by executing the programme specified by "Plan", the team of players will arrive at $a$ solution. Although a task like "Purchase raw materials" may result in an optimal solution, this solution will always be based upon information generated by tasks for which no optimizing programme has been found because the subproblems that correspond to these tasks involve a large degree of uncertainty (for example, unknown price elasticities) and/or equivocality (for instance when players disagree about what is a good marketing strategy). In other words, we have structured the task of playing a round of VUMAS in the procedure-oriented sense, but not in the model-oriented sense (see Section 2.2). Because of the equivocalities in the problem situation, a team will follow a satisficing approach of continuous planning (meanwhile recording promising solutions) until time is up and decision forms have to be handed over to the game manager. There are of course several criteria by which alternative solutions can be judged, such as expected profit, risk involved, flexibility of the work force, etcetera. In order to determine whether a solution is promising, an evaluating task like the one depicted in Figure 7-22 should be performed each time the planning task has been completed.

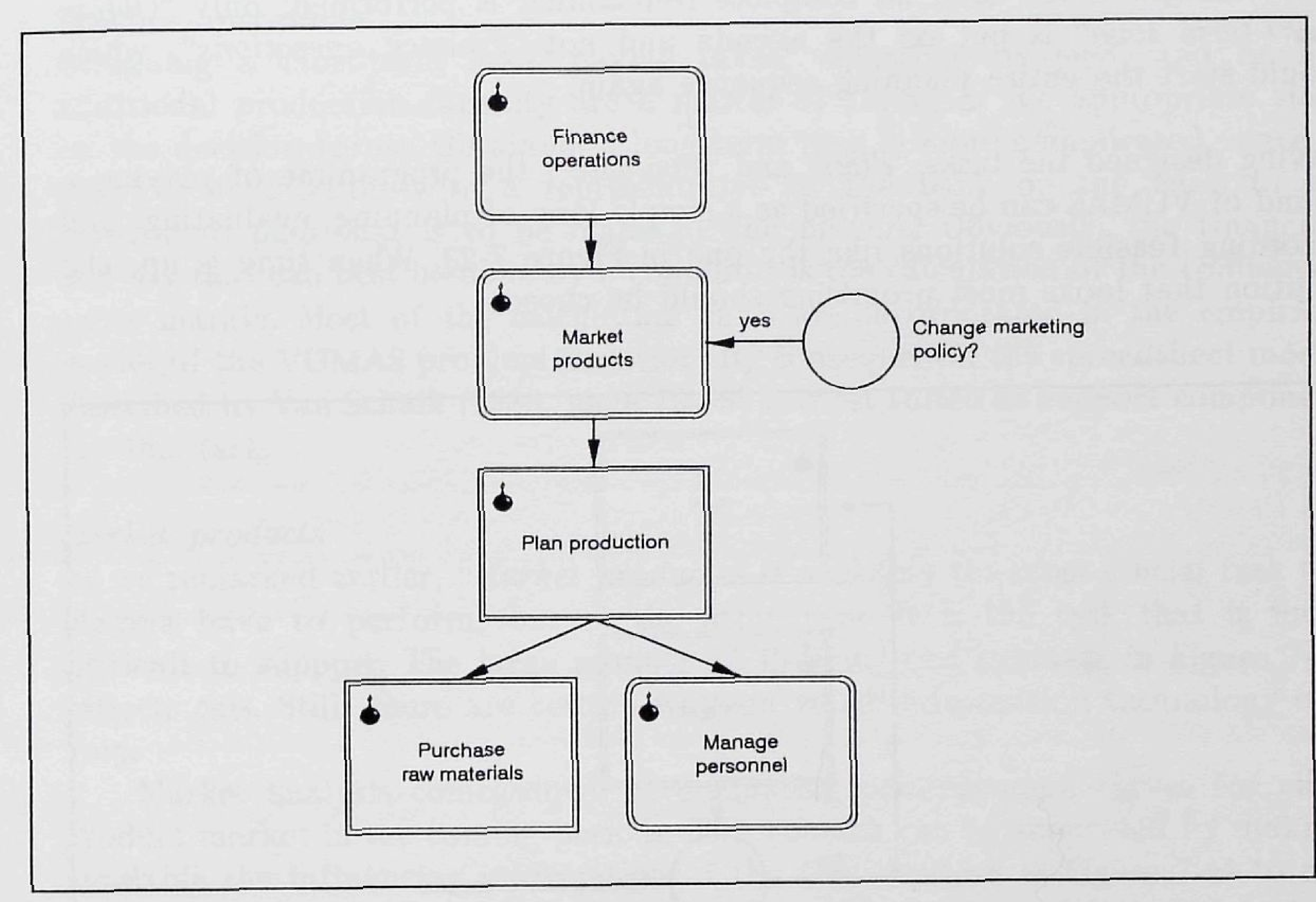

Flgure 7-21. A task structure for "Plan"

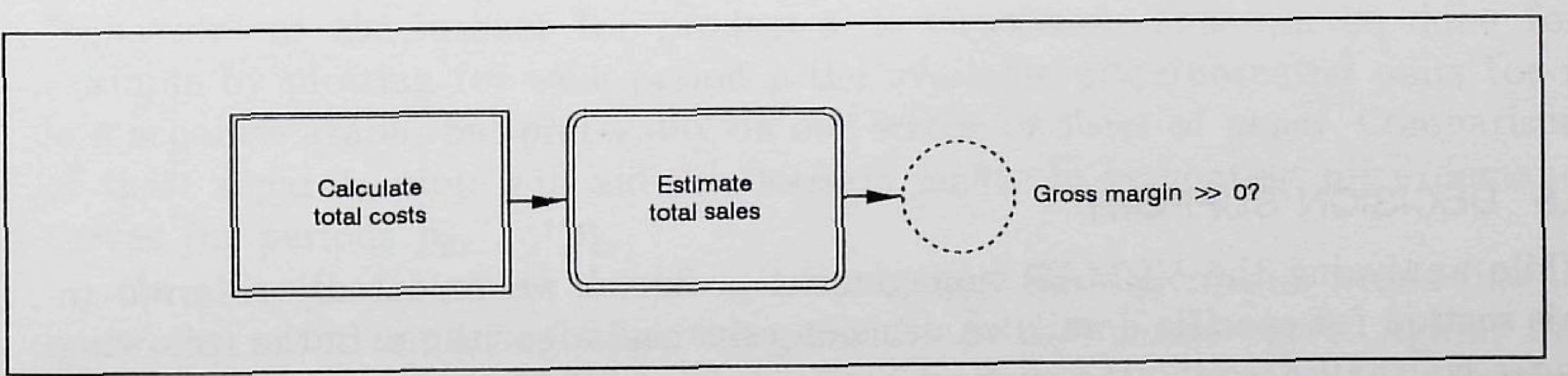

Fgure 7-22. A task structure for "Evaluate"

Our design of "Evaluate" reflects a strong emphasis on the profit aspect: A plan is evaluated by calculating the total costs and estimating the sales. Figure 7-22 shows that "Evaluate" signals the coordinating decision "Gross margin $\gg 0$ " in the financing task. By means of this coordinating mechanism we ensure that possible liquidity shortages are covered by short-term loans. Note that when the 
gross margin is too low, no complete re-planning is performed: only "Obtain short-term loan" is put on the agenda and not "Finance operations", which would start the entire planning sequence again.

Having designed the tasks "Plan" and "Evaluate", the programme of playing a round of VUMAS can be specified as a simple loop of planning, evaluating, and recording feasible solutions like the one in Figure $7-23$. When time is up, the solution that looks most promising should be chosen.

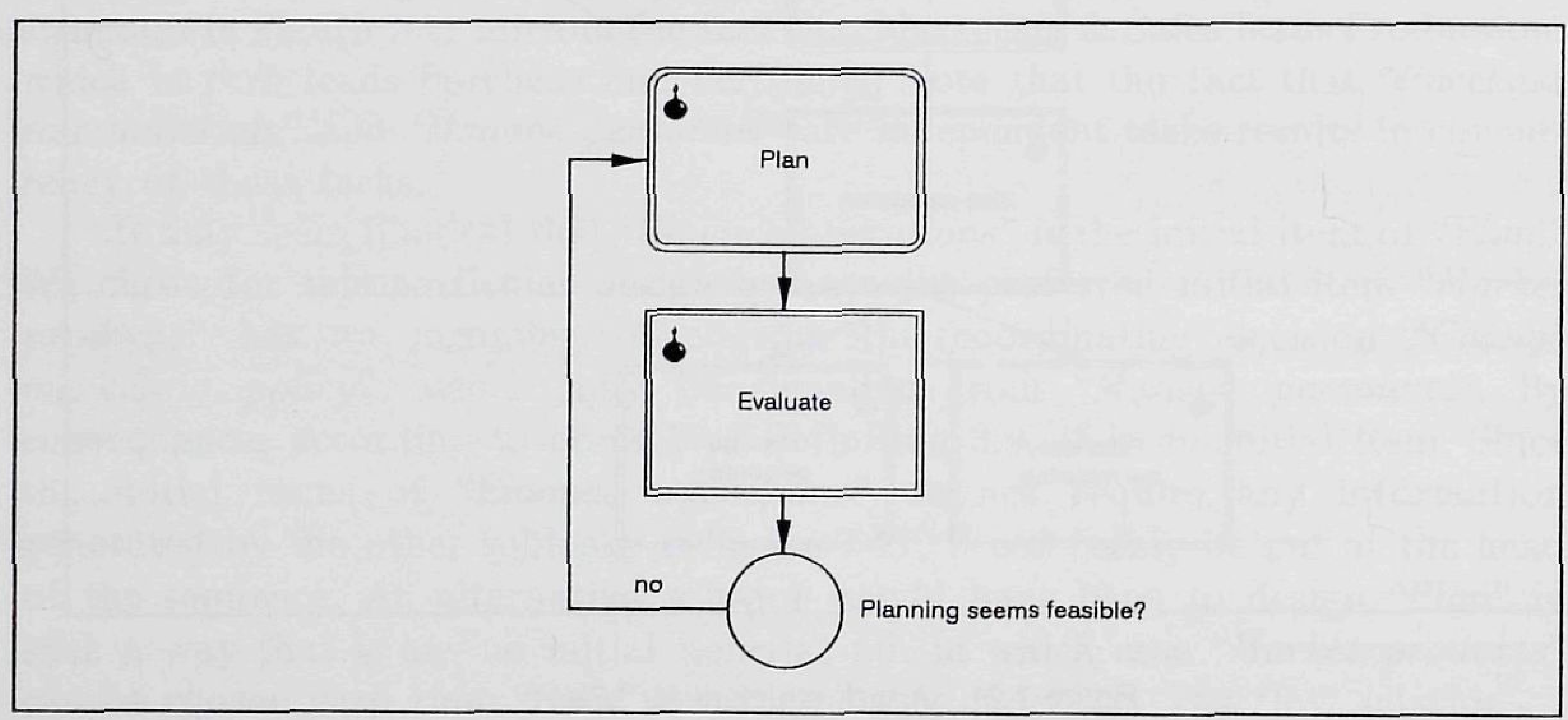

Figure 7-23. A task structure for "Play one round of VUMAS"

\subsection{DECISION SUPPORT}

While analyzing the VUMAS management problem, we repeatedly referred to this section for specific details on decision rules and algorithms. In the following pages we shall describe the support component of "Play one round of VUMAS" by making something like a depth-first traversal through its task hierarchy, meanwhile specifying how coordinating decisions and leaf tasks can be supported.

We do not pretend that the set of decision rules and models presented in this section is complete. After all, the primary purpose of this case study is not to design a DSS, but to test whether task analysis can help in finding a gaming strategy if we base ourselves on the VUMAS users manual only.
Finance operations

Obtaining a short-term loan, paying taxes, declaring dividend, and buying additional production capacity are a matter of filling in the appropriate slots on the decision forms. Obtaining a long-term loan is more complicated, since it requires negotiation with a representative of the bank on the basis of an investment plan that is to be made by the players. Obviously, the financing activity that can best be done by a computer is the calculation of the company's gross margin. Most of the calculation rules are incorporated in the empirical model of the VUMAS problem situation. By consequence, the spreadsheet model described by Van Schaik (1988, page 79-85) is most suited as support component for this task.

Market products

As we remarked earlier, "Market products" is probably the most crucial task the players have to perform, but at the same time it is the task that is most difficult to support. The large number of ill-structured subtasks in Figure 7-19 reflects this. Still, there are certain ways in which information technology can help.

Market analysis comes down to estimating price/demand curves for each product market in the coming periods. This subtask can be supported by making available the influencing information of the first decision in Figure 7-11 to the decision maker. The behavior of competitors will of course remain guesswork, but historical data on competing firms and price/potential data for future periods can be purchased from the research institute. A decision maker will be greatly helped if all available data is presented in such a way that the "behavior" of the market for product $x$ is visualized. This can be done for example by plotting for each period $p$ the available price/potential pairs for $x$ in a separate graph, but preferably on one screen or sheet of paper. Comparison of these separate plots will aid the decision maker in estimating price/demand curves for periods $p_{0}, \ldots, p_{4}$

In order to determine the advertising budget for $\mathrm{Abyco}$, one would like to know the exact impact of advertising on the price/demand curve. Again, the price/potential plots can be used in combination with data on advertising expenditures obtained from the research institute.

Figure 7-11 shows what information is required to determine a good selling price for a product. Assuming that a fair estimate of the price/demand curves for period $p_{0}$ and $p_{1}$ has been made, a fairly straightforward calculation tool can help to avoid mistakes such as selling for less than the actual cost price, and offering more than is in stock. It is also possible to construct a tool that calculates whether it is advantageous to stock instead of sell in view of the planned production, the price/demand curves, and the liquidity in periods $p_{0}$ and $p_{1}$. 
We find little computer support for the task of changing the company's product mix as a response to a changing market. An optimal product mix cannot be determined by means of for example linear programming because neither selling price nor production costs can be expressed in terms of linear equations. In order to support the last decision of Figure 7-11, it might be practical to develop a simple calculation tool that takes as input a product mix expressed in percentages (like 50\% Ethelite, 30\% Aminol Quality, and 20\% Abyco) and the company's total production capacity (for example, 4500 boiler hours), and returns as output the production level per product, expressed in tons.

\section{Plan production}

To support the planning of boiler hours we only need to extend the last of the Marketing \& Sales tools mentioned in the previous paragraph in such a way that a product mix can be input not only in percentages, but also in tons. The total production capacity then becomes an output parameter that indicates whether additional Stage I or Stage II capacity is needed.

Purchase raw materials

The initial task "Determine raw material requirements" can be fully automated by means of the following algorithm (see also Figure 7-7 on page 187):

\section{for $r m$ in (ATHEX, BYThOL, COPRAN) do}

$\mathrm{rmr}[\mathrm{rm}]:=0$;

for each product $p$ do $r m r[r m]+:=\operatorname{ppv}[p]$ * p. Composition $[\mathrm{rm}]$ od

\section{od;}

The four other subtasks (each with alias "Purchase one kind of raw material") are more demanding. The subtasks of this task require no support other than a pencil to write the actual amounts on the decision form that is to be handed in at the end of a round. The coordinating decisions, however, could use somewhat more sophisticated support.

The first decision requires a tool that checks whether a contract for raw material $r m$ exists (in which case no new contract can be initiated) and whether a new contract would be advantageous in view of the expected price of $r m$ in the next four periods. A simple heuristic decision rule is to initiate a new contract whenever the total amount of $\mathrm{rm}$ required in the next four periods exceeds 20 tons and the current price is less than or equal to the expected price in every one of those periods.

The second decision requires a tool that generates and compares various direct purchase alternatives in order to determine whether it is advantageous to purchase more than is needed in the current period. A heuristic for this decision would be to stock whenever the price in period $p_{0}$ is lower than the expected price in $p_{1}$ plus warehousing costs, and higher than the expected price in $p_{2}$. If, for example, the prices of Athex for $p_{0}, \ldots, p_{4}$ equal 19, 21, 17, 20, and 19, respectively, and the quantities required equal $10,11,10,12$, and 9 tons, the obvious choice would be to make a direct purchase of 21 tons in $p_{0}$, none in $p_{1}$, 10 in $p_{2}$ (at the same time initiating a contract for $4 \times 9$ tons for $p_{3}$ through $p_{6}$ ), 2 in $p_{3}$ and again none in $p_{4}$.

These heuristic decision rules are merely a matter of common sense. Still, the two coordinating decisions of "Purchase raw materials" can be supported even better with a tool that solves the following optimization problem:

Let $\mathrm{R}_{\mathrm{i}}$ be the quantity of raw material $\mathrm{rm}$ required in period $p_{\mathrm{i}}$, $\mathrm{P}_{\mathrm{i}}$ the price of raw material $\mathrm{rm}$ required in period $p_{\mathrm{i}}$,

$p_{c}$ the period in which a new contract is initiated,

$\mathrm{C}_{\mathrm{i}}$ the quantity $\mathrm{rm}$ that will be delivered on contract in period $p_{\mathrm{i}}$,

$\mathrm{D}_{\mathrm{i}}$ the quantity $\mathrm{rm}$ that is bought directly in period $p_{\mathrm{i}}$.

The stock level $S_{i}$ at the end of period $p_{i}$ then is defined by:

$$
S_{i}=S_{i-1}+C_{i}+D_{i}-R_{i}
$$

The objective is to minimize $\sum_{\mathrm{i}=1}^{4} \mathrm{~S}_{\mathrm{i}} * \mathrm{~W}+\mathrm{D}_{\mathrm{i}} * \mathrm{P}_{\mathrm{i}}+\mathrm{C}_{\mathrm{i}} * \mathrm{P}_{\mathrm{c}}$ while respecting the following conditions:

1. $\mathrm{R}_{\mathrm{i}}$ and $\mathrm{P}_{\mathrm{i}}$ are given, and cannot be changed;

2 . if some contract for $r m$ has been initiated in period $p_{\mathrm{j}}(-4<\mathrm{j}<1), \mathrm{C}_{\mathrm{i}}$ is given and cannot be changed for $i=1, \ldots, j+4$, and $c$ must be greater than $\mathrm{j}+3$;

3. if $\mathrm{c} \in\{0, \ldots, 3\}$ then $\mathrm{C}_{\mathrm{c}+1}=\mathrm{C}_{\mathrm{c}+2}=\mathrm{C}_{\mathrm{c}+3}=\mathrm{C}_{\mathrm{c}+4} \geq 5000$;

4. $\mathrm{S}_{\mathrm{i}} \geq 0$ for all $\mathrm{i}$;

The game rules require that quantities $C_{i}$ and $D_{i}$ are specified in integer units of 1000 kilograms. Moreover, these quantities have a lower bound of 0 , while minimization implies that $\mathrm{S}_{4}$ must be as close to 0 as is possible. Consequently, the number of alternatives is reasonably small, and an optimum can be found quickly by means of a simple generate-and-test algorithm.

\section{Manage personnel}

As can be seen in Figure $7-10$, the PSI plays an important role in personnel management, since it determines the actual number of operational workers. Wage level, Gratuities, and Size of the company's two work forces are entirely under control of the decision makers. Actions concerning these attributes take effect immediately, and this effect is fully predictable. Unfortunately, competing firms have a similar flexibility. Since the PSI strongly depends on the PSIs of 
competing firms, it is virtually impossible to give a correct estimate of the average PSI (and therefore one's own PSI) for the coming period. This seriously complicates the personnel management task.

In view of the large degree of uncertainty (not equivocality, since the dependencies between variables are known and deterministic) the support component of "Manage personnel" should allow the decision maker(s) to compare alternatives on several criteria: the expected cost, the risk that manning requirements cannot be met, and the consequences for future periods. For this purpose, at least three tools are needed:

1. a "PSI calculator" that shows what the company's PSI will be under given circumstances (Figure 7-4 gives an impression of what the output of this tool might look like);

2. a "surplus calculator" that computes the actual size of the company's two work forces for a given PSI;

3. a "cost calculator" that computes the total amount of money that is spent on wages, gratuities, hiring new operators, "paying off" labor unrest, etcetera.

These tools should be integrated in such a way that comparison of costs and surplus for various sets of input data is possible. Still, no matter how fast or accurate these tools function, the validity of their output will always depend on the estimated values of the PSI average of the competing firms.

\section{Evaluate}

It will be clear that evaluation of a plan requires an overview of the cost and risk estimates of each of the five departments. More specific support can probably be found by applying task analysis to "Evaluate" itself. Since the VUMAS users manual alone does not provide sufficient information to determine what constitutes a good evaluation strategy, a task structure for "Evaluate" cannot be designed following the "determine decision structuredesign task structure" approach for unknown problem situations (see page 63). This information can only be obtained by actually playing the game. We then can take the other approach ("determine task structure-analyze task structuredesign new task structure"). Since this would lead us beyond the scope of this chapter, we do not address the issue of decision support in any more detail.

\subsection{ADAPTIVITY}

After analyzing the task "Play one round of VUMAS", we have tested the adaptivity of its task structure by simulating crisis situations such as strikes, raw material shortage, machine breakdowns, "stunting" competitors, and opportunities such as "booming" markets or sudden drops in the prices of raw materials. In this section we will illustrate how new task structures are derived from an existing one with only slight modifications of its flow relation and coordinating mechanism.

In general, when a crisis occurs, the following three steps must be taken to ensure that the problem-solving programme specified by the task structure of "Play one round of VUMAS" is still appropriate:

1. check whether the crisis is at all noticed when the current task structure is executed;

2. for each task in the task hierarchy, see whether it specifies all of the subtasks and/or decisions actions that seem necessary to deal with the crisis, meanwhile checking whether the current coordinating mechanisms (in particular sequences and signals) need to be modified;

3. check whether new decision support is required.

In other words, we say that the current task structure can be used as a point of departure for the analysis of the new problem situation.

As an example, consider a situation in which there is a sudden and complete shortage of the raw materials Athex and Diox. This shortage is expected to last for three to five periods. Since Athex/Diox is essential to the manufacturing of each of the five products (see Figure 7-3 on page 184), production will be limited by the current stock level of Athex and Diox until new quantities can be purchased.

The first step in the analysis of this crisis situation is to check whether the task structure described in Section 7.4 contains some coordinating mechanism that can handle a sudden shortage of raw materials. If we look at the flow diagrams in Figure 7-15 and 7-16, we see that this is not the case. The obvious solution is to add a coordinating decision "Shortage?" to the task "Purchase raw materials". This new decision should be put on the agenda by any of the four tasks with alias "Purchase one kind of raw material". If a shortage occurs, "Shortage?" should signal a decision at some other coordination level that determines what action should be taken.

The next step is to reconsider the coordinating mechanisms. Obviously, the shortage means that the boiler planning must be completely revised. A shortage of raw materials means lower production levels, which in turn calls for a completely different marketing policy. Before Marketing \& Sales can determine appropriate selling prices, it must have a good overview of the various product mixes (in tons) that are still possible. The decision structure of "Plan" shows that this information ("Maximal production levels in $p_{0}$ " in Figure 7-9 and 7-11) 
is generated by Production. But the current coordinating mechanism of "Plan production" will signal a lack of production capacity only. A possible solution is to identify the calculation of maximal production levels as a separate subtask of "Plan production", and to have it put on the agenda whenever a shortage occurs. We therefore add the coordinating decision "Production limits expected?" to the task "Plan production", and add a flow labeled "yes" from this decision to the new subtask "Calculate maximum production levels". Next, we create a signal construction by adding a flow labeled "yes" from "Shortage?" to "Production limits expected?". Finally, we add a flow from "Calculate maximum production levels" to the coordinating decision "Change marketing policy?" in Figure 7-21 to ensure that the shortage and the resulting production constraints are noticed by Marketing \& Sales.

The third step is to determine whether additional support is needed. Since we identified "Calculate maximum production levels" as a new task, this task is an obvious candidate for additional support. One can think of a tool that produces a table with all possible product mixes (since the total production volume is limited by the inventory levels, and since boilers are planned in units of 250 hours, the number of possible combinations will be manageable), and for each mix the estimated production costs.

\subsection{SUMMARY AND CONCLUSIONS}

In this chapter we reported on our analysis of the problem-solving process of several decision makers working on different but related problems. We presented a detailed conceptual model of the problem situation faced by a team of players of the management game VUMAS, and indicated how this conceptual model can be "translated" into an equation-type empirical model that is easily implemented in any commercially available spreadsheet package. We were able to design a detailed gaming strategy by applying task analysis to the problem as it is stated in the game's users manual.

Like we did at the close of the previous two chapters, we divide our conclusions in two categories. Those in the first category again relate to our approach to problem solving in general, based on our further experiences with objectoriented modeling and our task analysis technique. Those in the second category relate to the gaming strategy we have developed for the VUMAS management game.

\section{The problem-solving approach}

Firstly, we conclude that it is possible to describe related and possibly concurrent problem-solving processes using our task analysis technique.
Secondly, we found that the decision structures for the five subproblems and the integral task structure constitute a sound basis for the development of a DSS that is based primarily on a decision process, and not on whatever happens to be the newest available technology. Using the phrase coined at the end of Section 1.2, the task structure and its support component together constitute an information system to support a set of decision processes.

Finally, from our analysis of the raw materials shortage and other "crises" we conclude that a task structure is a very flexible representation of a problemsolving programme, provided that an adequate "task analysis support kit" is available. Although our experiments with the Smalltalk prototype described in Chapter 4 have been limited, they clearly indicated that the integration of task structures and conceptual and empirical models in a full-fledged PSSE wil provide the means to experiment with different scenarios for the same crisis situation before chosing one.

II. The gaming strategy

By first determining a decision structure for each of the five departments mentioned in the users manual, and subsequently designing a task structure that incorporates all of the identified decisions, is consistent with the precedence relation, and solves detected coordination problems, we have obtained a programme for playing a round of VUMAS. On page 188 we made reference to the research by Van Schaik (1988), who used this management game to test the effectiveness of spreadsheet-type decision support systems. His experiments also involved the use of a particular gaming strategy, which is depicted as a flowchart in Figure 7-24. We realize that this strategy is by no means an absolute standard. But since it is has been developed by experts on the VUMAS game, comparison with our task structure from Section 7.4 will at least provide some indication of the validity of this task structure.

Note that the global structure of the two programmes is almost identical: Marketing \& Sales leads Production, which in turn leads Purchase and Personnel. Cost calculation and financing considerations may call for a revision of the marketing policy.

The main difference lies in the level of detail. The flowchart in Figure 7-24 gives no indication whatsoever with respect to the kind of information that should be used. Another important difference is that Figure 7-24 mirrors a "true" satisficing approach in the sense that the first plan that seems feasible is implemented, while the flow diagram in Figure $7-23$ reflects more of a generate-and-test approach. A third difference relates to the computerized support that is offered to the players: The spreadsheet-based DSS described by Van Schaik does not provide any of the specific tools for market analysis, purchase, and personnel management described in Section 7.4. 
In view of these differences, it would be interesting to conduct an experiment similar to those reported by Van Schaik (1988) and Bots, Van Schaik and Sol (1989), this time using the task structure and computer support described in this chapter.

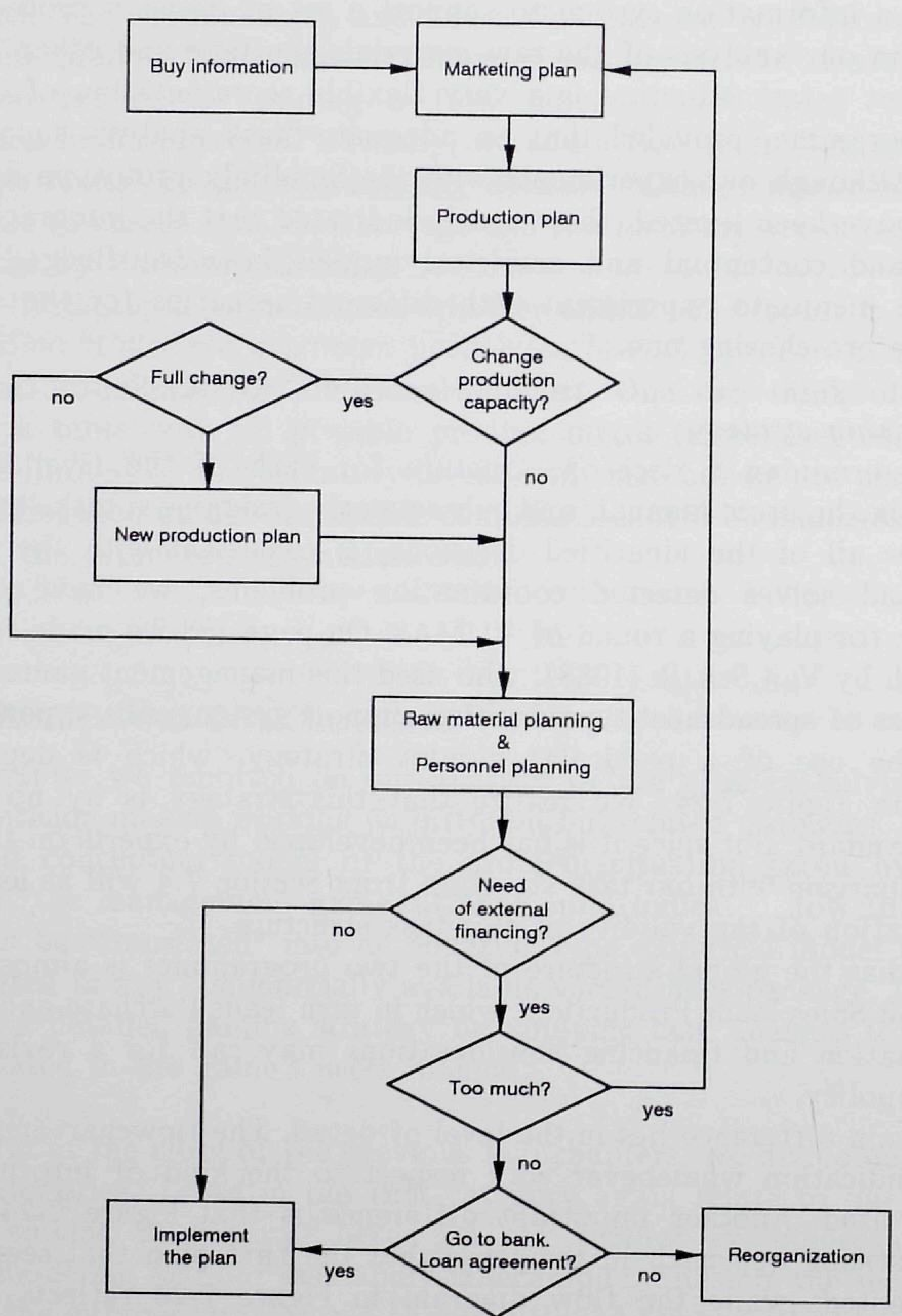

Fgure 7-24. The decision strategy proposed by Van Schaik (1988, page 89 )

\section{EPILOGUE}

If it were done when 't is done, then 't were well. - William Shakespeare

\subsection{INTRODUCTION}

In this thesis we have addressed the issue of improving the performance of an organization by means of information technology. We observed that decision making has become the primary activity in organizations, and that information technology is applied to support human beings in their problem-solving tasks, regardless whether this technology is called MIS, DSS, OIS, EIS, or something else. This observation stimulated us to approach the issue of performance improvement from a problem-solving point of view. The overall objective was to find a way to integrate problem-solving processes and information technology.

We based our research on the supposition that the performance of an organization mainly depends on the limited problem-solving capabilities of the individuals that are part of that organization, and on the coordination of their problem-solving processes. Based on this premise we defined a technique for formulating and structuring problems that combines the concepts problem, problem structure, task, decision, information, coordination, and computer support in a consistent way. Next, we hypothesized that this technique leads to improvement of organizational performance through proper application of information technology. The larger part of this thesis reports on how we tested this "refutable" theory. In this final chapter we review the five hypotheses that made up this theory, and assess the extent to which they are upheld by our findings.

\subsection{A REVIEW OF HYPOTHESES}

Recall that the process of problem solving is viewed as an interplay of four stages: conceptualization, specification, solution finding, and implementation. Conceptualization involves identifying the classes of objects that play a part in the problem situation, and describing their properties, their possible actions, and the way they relate to other object classes. The resulting set of class definitions constitutes a conceptual model that describes the problem situation in broad terms. During the specification stage, the problem situation is described in detail by defining artefacts (if necessary), creating instances of object classes, and assigning appropriate initial values to their attributes. This results in an 
empirical model. Conceptualization and specification are repeated until the empirical model shows sufficient correspondence with reality. Solution finding is viewed as designing a task structure that describes how the problem can be solved. The advantages and disadvantages of alternative task structures can be estimated by testing them within the empirical model. Once an adequate task structure has been designed, implementation comes down to actually "executing" this task structure in reality. The crux of this view on problem solving is expressed by the first hypothesis:

\section{Hypothesis 1.}

In the process of problem solving one should distinguish between problem structure and task structure.

As has been explained, "problem structure" refers to a set of variables, relations, operations, and evaluation criteria. This set is made explicit by means of a conceptual and an empirical model. These models give no indication as to what course of action should be taken, but they specify the boundaries within which a solution can be looked for. Their purpose is to reduce the cognitive limitations of the decision makers by giving them a better understanding of the problem situation. By contrast, a task structure specifies a course of action; it provides little or no information on what the effect of this course of action will be.

In Chapter 3 we have shown that it is possible to define a set of concepts and construction rules that allow for a sharp distinction between problem structure and task structure. The demarcation rule proves to be a useful instrument for delineating both problem boundaries and the scope of the solution that is eventually chosen. Chapter 4 confirms the feasibility of an object-oriented environment that supports the construction, integration, and evaluation of models and task structures. The case studies reported in Chapter 5,6 , and 7 indicate that an approach of first aiming for a good understanding of the problem by describing its structure (and if possible the problem-solving programme that has been used so far) and only then focus on finding a solution is quite effective. On these grounds, Hypothesis 1 cannot be rejected.

The next two hypotheses are essential to the theory that tasks are problemsolving processes that can be specified as such:

\section{Hypothesis 2 .}

Tasks and coordinating mechanisms can be specified in terms of decisions that are to be made.

\section{Hypothesis 3.}

Decisions can be specified in terms of the information they require and generate.
In Chapter 3 we have demonstrated that it is possible to define "task" and "decision" in such a way that a specification of tasks and coordinating mechanisms in terms of decisions is possible. Our formal definition of "decision" agrees with Hypothesis 3, while the task structures presented in Chapter 4 through 7 demonstrate that the definitions of "task" and "decision" offer sufficient expressive power and flexibility to represent a variety of problemsolving programmes and coordinating mechanisms. Consequently, neither Hypothesis 2 nor Hypothesis 3 can be rejected.

The fourth hypothesis relates to the feasibility of an environment to support problem solving:

\section{Hypothesis 4.}

The construction of conceptual and empirical models, and the design and analysis of task structures can be adequately supported by information technology that is currently available.

The analysis of the task "Solve problem" revealed no need for support that cannot be met by means of state-of-the-art information technology. The feasibility of an object-oriented dynamic modeling environment and a "task analysis support kit" has been demonstrated. Experiments with the prototype PSSE in Smalltalk/V have confirmed that conceptual and empirical models and task structures can be integrated in such a way that alternative programmes can be evaluated by "executing" the corresponding task structures within the empirical model. In other words, Hypothesis 4 is also upheld by our findings.

From an information systems designer's perspective, the fifth and last hypothesis is perhaps the most important one:

Hypothesis 5 .

Every task structure constitutes a "blueprint" for a task-specific information system.

In view of the results of the case studies reported in Chapter 5 and 6 , this hypothesis cannot be rejected. The analysis of the task of making individual rosters has resulted in a task structure that could be translated directly and at low cost into a supporting information system that is tailored to a personnel manager's decision process. The analysis of the problem of international transfer pricing in the pharmaceutical industry has resulted in a group decision support system. Finally, the analysis described in Chapter 7 has yielded not only a gaming strategy, but also the specifications of a set of supporting software tools for each of the subtasks of "Play one round of VUMAS". Each of these tools constitutes a small, task-specific information system that can be implemented easily with the help of a financial modeling package. 


\subsection{CONCLUDING REMARKS}

Since none of the hypotheses that make up the "refutable" theory formulated at the end of Chapter 2 is rejected, we can state that this theory is upheld by our findings. Presently, we wish to add a few mitigating comments to this conclusion.

First of all, we emphasize that we have demonstrated the feasibility of a single-user PSSE. Although the support requirements that have been determined by analyzing the task "Solve problem" in Chapter 4 are essentially independent of the number of decision makers involved, a distributed, multi-user PSSE will require more sophisticated technology than what we have used in our research. Still, we dare to state without evidence that these technological requirements are not beyond the current state of the art.

Secondly, we are well aware of the fact that the case studies reported in the preceding chapters deal with relatively small problems. It remains to be seen whether we can deal as successfully with problems involving a hundred or more individuals.

We wish to conclude with the remark that there remains a substantial gap between solution finding and implementation. Frequently, there will be financial, organizational, political, or other reasons why a promising task structure cannot be implemented in an organization. This is not a shortcoming of the problemsolving approach described in this thesis. A PSSE and the underlying theory provide a base for "understanding before design": Constructing an object representation and applying task analysis to the current organizational decision processes results in a proper understanding of the problem situation, which precedes the design of new task structures and supporting software. This understanding will always be gained, regardless whether the subsequent design is successful. We hypothesize that the models for understanding provide decision makers with a yardstick for actually measuring the change in organizational performance. Obviously, testing this hypothesis will be a subject for future research.

\section{REFERENCES}

Il y a plus affaire à interpréter les interprétations qu'à interpréter les choses, et plus de livres sur les livres que sur autres subjects. Nous ne faisons que nous entregloser
- Montaigne

AcKoff, R. L., "The Art and Science of Mess Management", TIMS Interfaces, Vol. 11, No. 1 (February 1981), pp. 20-26.

Aiello, L., D. NARdi and M. PANTi, "Structural Office Modeling: A First Step Towards the Office Expert System", Proceedings of the $2^{\text {nd }} A C M$ Conference of Office Information Systems, Toronto, June 1984.

Alter, S. L., "A Taxonomy of Decision Support Systems", Sloan Management Review, Vol. 19, No. 1 (Fall 1977), pp. 39-55.

Amarel, S., "On the Mechanization of Creative Processes", IEEE Spectrum, Vol. 3 (April 1966), pp. 112-114.

AsaI, K. and H. TanaKa, "Applications of Fuzzy Sets Theory to Decision Making and Control", Journal of AACE, Vol. 19 (1975), pp. 235-242.

AW-V, General Industrial Employers' Association, Algemene WerkgeversVereniging, Haarlem, the Netherlands, September 1987.

BARBER, G. R., "Supporting Organizational Problem Solving with a Workstation", ACM Transactions on Office Information Systems, Vol. 1, No. 1 (January 1983), pp. 45-67.

Barr, A. AND E. A. Feigenbaum (EDs.), The Handbook of Artificial Intelligence, Vol. I, William Kaufman, Los Altos, CA, 1981.

BeLew, R. K., "Evolutionary Decision Support Systems", in L. B. Methlie and R. H. Sprague (Eds.), Knowledge Representation for Decision Support Systems, Elesevier Science Publishers (North-Holland), Amsterdam, 1985.

BennetT, J. L., Building Decision Support Systems, Addison-Wesley, Reading, MA, 1985

Ben-Bassat, M. "Expert Systems for Clinical Diagnosis", in M. M. Gupta, A. Kandel, W. Bandler and J. B. Kiszka (Eds.), Approximate Reasoning in Expert Systems, Elsevier Science Publishers (North-Holland), Amsterdam, 1985, pp. 671-687.

Bonczek, R. H., C. W. Holsapple and A. B. Whinston, Foundations of Decision Support Systems, Pergamon Press, New York, NY, 1981.

Bosman, A., "DSS, a Discipline or a Vision", in H. G. Sol, C. A. Th. Takkenberg and P. F. De Vries Robbé (Eds.), Expert Systems and Artificial Intelligence in Decision Support Systems, Proceedings of the Second Mini Euroconference, Lunteren, the Netherlands, November 1985, pp. 23-39. 
Bosman, A., "Ontwerpen, Procesmodellen en Beslissen", Informatie, Vol. 28 No. 4 (April 1986), pp. 310-321 (in Dutch).

Bosman, A. AND H. G. Sol, "Knowledge Representation and Information Systems Design", in L. B. Methlie and R. H. Sprague (Eds.), Knowledge Representation for Decision Support Systems, Elsevier Science Publishers (North-Holland), Amsterdam, 1985, pp. 81-91.

Bots, P. W. G. AND H. G. Sol, "An Environment to Support Problem Solving", Decision Support Systems, Vol. 3, No. 3 (December 1987), pp. 225-231.

Bots, P. W. G. AND H. G. Sol, "Shaping Organizational Information System through Co-ordination Support", in R.M. Lee, A.M. McCosh and P. Migliarese (Eds.), Organizational Decision Support Systems, Elsevier Science Publishers (North-Holland), Amsterdam, 1988, pp. 139-154.

Bots, P. W. G., F. D. J. Van Schaik and H. G. Sol, "A Gaming Environment for Testing Decision Support Systems",, in D. Murray-Smith, J. Stephenson and R. N. Zobel (Eds.), Proceedings of the $3^{\text {rd }}$ European Simulation Congress, Edinburgh, Scotland, September 1989, pp. 673-678.

Bracchi, G. and B. Pernici, "The Design Requirements of Office Systems", ACM Transactions on Office Information Systems, Vol. 2, No. 2 (April 1984), pp. 151-170.

Brown, J. S., Applications of Artificial Intelligence, MIT Press, Cambridge, MA, 1983.

BRUSSAard, B. K. AND P.A. TAs, "Information and Organization Policies in Public Organization", in S.H. Lavington (Ed.), Information Processing 80, NorthHolland Publishing Company, Amsterdam, The Netherlands, 1980, pp. 821 826.

Bush, V., "As We May Think", Atlantic Monthly, Vol. 176, No. 1 (July 1945), pp. 101-108.

CAPra, Fritjof, The Turning Point, Bantam Books, New York, NY, 1983, p. 295.

CARPhen, CARIL, The Devil Princes, Elucidarian Press, New Wexford, Aloysius, Wega. Imaginary author, quoted by Jack Vance in Chapter 10 of Star King, Berkley Books, New York, NY, 1964.

Carpano, M. J., "Automatic Display of Hierarchized Graphs for Computer-Aided Decision Analysis", IEEE Transactions on Systems, Man, and Cybernetics, Vol. 10, No. 11 (November 1980), pp. 705-715.

CARroll, Lewis, "Punctuality", The Humorous Verses of Lewis Carroll, Dover Publications, New York, NY, 1960, pp. 4-5.

ChandRaseKaran, B., "Expert Systems: Matching Techniques to Tasks", in W. Reitman (Ed.), Artificial Intelligence Applications for Business, Abley, Norwood, NJ, 1984, pp. 41-85.

CHILD, J., Organizations: A Guide to Problems and Practice, Harper \& Row, New York, N.Y., 1977.
Cox, B., Object-Oriented Programming: An Evolutionary Approach, AddisonWesley, Reading, MA, 1986.

Croft, W. B. And L. S. Lefkowitz, "Task Support in an Office System", $A C M$ Transactions on Office Information Systems, Vol. 2 No. 3 (July 1984), pp. 197-212.

Daft, R. L. AND R. H. Lengel, "Organizational Information Requirements, Media Richness and Structured Design", Management Science, Vol. 32, No. 5 (May 1986), pp. 554-571.

Dahl, O. J., B. Myhrhaug and K. NygaArd, SIMULA 67 Common Base Language, NCC Publication S-52, Norwegian Computer Center, Oslo, Norway, 1970.

Dawson, K., "Rostering in the Process Industries", Management Services, December 1987, pp. 12-17.

DeMarco, T., Structured Analysis and System Specifications, Yourdon Inc., New York, NY, 1978

Digitalk, SMALLTALK/V: Tutorial and Programming Handbook, Digitalk Inc., Los Angeles, CA, 1986.

Dijkstra, E. W., "GOTO Statement Considered Harmful", Communications of the ACM, Vol. 11 (1968), p. 147

Donovan, J. J. AND S. E. MADNICK, "Institutional and Ad Hoc DSS and their Effective Use", Data Base, Vol. 8, No. 3 (Winter 1977), pp. 79-88.

Doyle, Arthur Conan, "The Adventure of the Dancing Men", see for example The Penguin Complete Sherlock Holmes, Hazel, Watson \& Viney, Aylesbury, Bucks, England, 1981, p. 511.

Eddings, David, Pawn of Prophecy, Ballantine Books, New York, NY, 1982, p. 86.

Einhorn, H. J. and R. M. Hogarth, "Behavioral Decision Theory: An Empirical Assessment of Decision Making", Decision Sciences, Vol. 17 (1986), pp. 79 91

Elam, J. J., J. C. Henderson, P. G. W. Keen, B. R. Konsynski, C. L. Meador and D. N. Ness, A Vision for Decision Support Systems, Unpublished manuscript, 1984.

ELLIS, C. A. AND M. BERNAL, "OFFICETALK-D: An Experimental Office Information System", in Proceedings of the ACM SIGOA Conference on Office Systems, Philadelphia, June 1982, pp. 131-140.

Ellis, C. A. AND N. NafFaH, Design of Office Information Systems, SpringerVerlag, Berlin, 1987.

Ellis, C. A. AND G. NuTt, "Office Information Systems and Computer Science", ACM Computing Surveys, Vol. 12, No. 1 (March 1980), pp. 27-60.

Flores, F., M. Graves, B. Hartfield and T. Winograd, "Computer Systems and the Design of Organizational Interaction", ACM Transactions on Office Information Systems, Vol. 6, No. 2 (April 1988), pp. 153-172.

Galbraith, J. R., Organization Design, Addison-Wesley, Reading, MA, 1977. 
GiBBs, S., "Office Information Models and the Representation of 'Office Objects", in Proceedings of the ACM SIGOA Conference on Office Systems, Philadelphia, June 1982, pp. 21-26.

GinzBerg, M. J. And E. A. Stohr, "DSS: Issues and Perspectives", in M. J. Ginzberg, W. Reitman and E. A. Stohr, Decision Support Systems, NorthHolland Publishing Company, Amsterdam, 1982, pp. 9-31.

GLuss, B., "Fuzzy Multi-stage Decision Making", International Journal of Control, Vol. 17 (1973), pp. 177-192.

GolDBERG, A., SMALLTALK-80: The Interactive Programming Environment, Addison-Wesley, Reading, MA, 1984.

GoldBerg, A. AND D. RoBson, SMALLTALK-80: The Language and its Implementation, Addison-Wesley, Reading, MA, 1983.

GorRy, G.A. AND M.S. ScotT MorTon, "A Framework for Management Information Systems", Sloan Management Review, Vol. 13, No. 1 (Fall 1971), pp. 55-70.

GuÉPIN, J. P., "Allemaal waanzin", in Allemaal raadsels, eh?, Amsterdam, 1974 (in Dutch).

Hackathorn, R. D., "Using Block Clustering to Analyze Information System Activity", in Proceedings of the $10^{\text {th }}$ Annual Hawaii International Conference on Systems Science, January 1977a.

HackathoRn, R. D., "Modeling Unstructured Decision Making", Data Base, Vol. 8, No. 3 (Winter $1977 b$ ), pp. 41-42.

HÄMmÄINEN, H., R. SUlONEN AND C. BÉRARD, "PAGES: Intelligent Forms, Intelligent Mail and Distribution", in G. Bracchi and D. C. Tsichritzis (Eds.), Office Systems: Methods and Tools, preprints of the IFIP WG8.4 Working Conference on Methods and Tools for Office Systems, Pisa, Italy, 22-24 October 1986, pp. 43-55

HewitT, C. E., "Offices Are Open Systems", ACM Transactions on Office Information Systems, Vol. 4, No. 3 (July 1986), pp. 271-287.

HirschHeim, R. A., "Understanding the Office: A Socio-Analytical Perspective", ACM Transactions on Office Information Systems, Vol. 4, No. 4 (October 1986), pp. 331-334.

Holbæk-Hanssen, E., P. HÅndlykken and K. NygaARd, System Description and the Delta Language, Norwegian Computing Center, Oslo, Norway, 1975.

Holloway, C. A., Decision Making under Uncertainty: Models and Choice, Prentice-Hall, Englewood Cliffs, NJ, 1979.

Holt, A. W., "Diplans: A New Language for the Study and Implementation of Coordination", ACM Transactions on Office Information Systems, Vol. 6, No. 2 (April 1988), pp. 109-125.

Holt, A. W. And P. Cashman, "Designing Systems to Support Cooperative Activity: An Example from Software Management", in Proceedings of IEEE Computer Society's $5^{\text {th }}$ International Computer Software and Applications Conference, IEEE Computer Society, Los Alamitos, CA, 1981, pp. 184-190.
HubER, G. P., "The Nature and Design of Post-Industrial Organizations", Management Science, Vol. 30, No. 8 (August 1984a), pp. 928-951.

Huber, G. P., "Issues in the Design of Group Decision Support Systems", Management Information Systems Quarterly, Vol. 8, No. 3 (September 1984b), pp. 195-204.

Huber, G. P. AND R. R. MCDAniel, "The Decision-Making Paradigm of Organizational Design", Management Science, Vol. 32, No. 5 (May 1986a), pp. 572-589.

Huber, G. P. AND R. R. McDaniel, "Exploiting Information Systems to Design More Effective Organizations", in M. Jarke (Ed.), Managers, Micros and Mainframes, John Wiley \& Sons, New York, NY, 1986b, pp. 221-236.

Jackson, M. A., Principles of Program Design, Academic Press, New York, 1975.

JACKY, J. P. AND I. J. KaRlet, "An Object-Oriented Programming Discipline for Standard Pascal", Communications of the ACM, Vol. 30, No. 9 (September 1987).

JACOB, J., "Roosters maken gaat de computer nog boven de pet", de Volkskrant, Saturday, March 5th, 1988, p. 35 (in Dutch).

JARKE, M., "Group Decision Support through Office Systems: Developments in Distributed DSS Technology", in E. R. McLean and H. G. Sol (Eds.), Decision Support Systems: A Decade in Perspective, Elsevier Science Publishers (North-Holland), Amsterdam, 1986, pp. 145-155.

Joyner, R. AND K. Tunstall, "Computer Augmented Organizational Problem Solving", Management Science, Vol. 17, No. 4 (December 1970), pp. 212225.

Kappel, G., A. M. TJoA and R. R. Wagner, "A Knowledge-Based Modelling Approach to Office Form-Flow Systems", in M. S. Elzas, T. I. Ören and B. P. Zeigler (Eds.), Modeling and Simulation Methodology in the Artificial Intelligence Era, Elsevier Science Publishers (North-Holland), Amsterdam, 1986 , pp. 365-373.

Keen, P. G. W., "Adaptive Design for Decision Support Systems", Data Base, Vol. 12, No. 1/2 (Fall 1980), pp. 16-25.

KeEn, P. G. W., "Value Analysis: Justifying Decision Support Systems", Management Information Systems Quarterly, Vol. 5, No. 1 (March 1981), pp. $1-15$.

KeEn, P. G. W., Competing in Time: Using Telecommunications for Competitive Advantage, Ballinger, New York, 1986.

Keen, P. G. W., "DSS: The Next Decade", Decision Support Systems, Vol. 3, No. 3 (December 1987), pp. 253-265.

Keen, P. G. W. And M. S. Scott Morton, Decision Support Systems, An Organizational Perspective, Addison-Wesley, Reading, MA, 1978.

KNuth, D. E., "Computer-Drawn Flowcharts", Communications of the ACM, Vol. 6, No. 9 (September 1963), pp. 556-563. 
Knuth, D. E., Sorting and Searching, Volume 3 of The art of Computer Programming, Addison-Wesley, Reading, MA, 1973.

Kung, C. H. AND A. Sølvberg, "Activity Modeling and Behaviour Modeling", in T. W. Olle, H. G. Sol and A. A. Verrijn-Stuart (Eds.), Information System Design Methodologies: Improving the Practice, Elsevier Science Publishers (North-Holland), Amsterdam, 1986, pp. 145-171.

Lafferty, R.A., "Primary Education of the Camiroi", in I. Asimov, M. Greenber and C. Waugh (Eds.), Young Extraterrestrials, Harper \& Row, New York, NY, 1984 , p. 132.

LaKatos, I., "Falsification and Methodology of Scientific Research Programmes", in I. Lakatos and A. Musgrave (Eds.), Criticism and the Growth of Knowledge, Cambridge University Press, Cambridge, UK, 1970.

LANGEFors, B., "Theoretical Analysis of Information Systems", BIT 3, 1963 pp. 229-254. Also appeared in J. D. Couger and R. W. Knapp (Eds.), System Analysis Techniques, John Wiley \& Sons, New York, NY, 1974.

LEE, R. M., "Bureaucracies as Deontic Systems", ACM Transactions on Office Information Systems, Vol. 6, No. 2 (April 1988), pp. 87-108.

LitTle, J. D. C., "Models and Managers: The concept of a Decision Calculus", Management Science, Vol. 16, No. 8 (April 1970), pp. B466-B485.

Lochovsky, F. H., "A Knowledge-Based Approach to Supporting Office Work", Database Engineering, IEEE Computer Society, Vol. 16, No. 3 (September 1983), pp. 174-182.

Lockemann, P. C., "Object-Oriented Information Management", Decision Support Systems, Vol. 5 (1989), pp. 79-102.

Lootsma, F. A., J. Meisner and F. Schellemans, "Multi-criteria Decision Analysis as an Aid to the Strategic Planning of Energy R\&D", European Journal of Operations Research, Vol. 25, No. 2 (1986), pp. 216-234.

LundeBerg, M., "The ISAC Approach to Specification of Information Systems and its Application to the Organization of an IFIP Working Conference", in T. W. Olle, H. G. Sol and A. A. Verrijn-Stuart (Eds.), Information Systems Design Methodologies: A Comparative Review, North-Holland Publishing Company, Amsterdam, 1982, pp. 173-234.

LundT, O. E., Planning of Shift Work, Association of Employers of the General Danish Industries, February 1984.

Maes, R., J. van Thienen and M. Verhelst, "The Procedural Decision Modelling System", in H. G. Sol (Ed.), Processes and Tools for Decision Support, North-Holland Publishing Company, Amsterdam, 1983, pp. 139-154.

MARCH, J. G., "Bounded Rationality, Ambiguity, and the Engineering of Choice", Bell Journal of Economics, Vol. 9, No. 2 (Autumn 1978), pp. 587-608.

MARCH, J. G. AND Z. SHAPIRA, "Behavioral Decision Theory and Organizational Decision Theory", in G. R. Ungson and D. N. Braunstein (Eds.), Decision
Making: An Interdisciplinary Inquiry, Kent Publishing, Boston, MA, 1982, pp. 92-115.

McFarlan, F. W., J. L. McKenney and P. Pyburn, "The Information Archipelago - Plotting the Course", Harvard Business Review, Vol. 61, No. 1 (January/ February 1982), pp. 145-156.

Mclean, E. R. And H. G. Sol (Eds.), Decision Support Systems: A Decade in Perspective, Elsevier Science Publishers (North-Holland), Amsterdam, 1986.

Meyer, B., Object-oriented Software Construction, Prentice-Hall, London, 1988. Milne, A. A., Winnie-the-Pooh, E.P. Dutton, New York, NY, 1984, page 50.

Miner, F. C., "A Comparative Analysis of Three Diverse Decision Making Approaches", Academy of Management Journal, Vol. 22, No. 1 (March 1979), pp. $81-93$.

MintzBeRg, H., The Nature of Managerial Work, Harper \& Row, New York, NY, 1973.

MintzBerg, H., The Structuring of Organizations, Prentice-Hall, Englewood Cliffs, NJ, 1979.

MintzBerg, H., Structure in Fives, Prentice-Hall, Englewood Cliffs, NJ, 1983.

MintzBerg, H., D. Raisinghani And A. ThÉorÊt, "The Structure of 'Unstructured' Decision Processes", Administrative Science Quarterly, Vol. 21, No. 2 (June 1976), pp. 246-275.

Mitroff, I. I. AND F. Betz, "Dialectical Decision Theory: A Meta Theory of Decision Making", Management Science, Vol. 19, No. 1 (September 1972), pp. 11-24.

Mitroff, I. I. ET AL., "On Managing Science in the System Age: Two Schemes for the Study of Science as a Whole Systems Phenomenon", TIMS Interfaces, Vol. 4, No. 3 (May 1974).

Mitroff, I. I. and F. R. Featheringhouse, "On Systematic Problem Solving and the Error of the Third Kind", Behavioral Science, Vol. 19 (1974).

Montaigne, Michel De, Essais, Livre II, Chap. 13, Éditions Pierre Villey, Paris, France, 1965, p. 1069

Moore, J. H. AND M. G. Chang, "Design of DSS", Data Base, Vol. 12, No. 1/2 (Fall 1980), pp. 8-14

NaisBitT, J., Megatrends, Warner Books, New York, NY, 1982.

NASSI, I. and B. ShNeiderman, "Flowchart Techniques for Structured Programming", Sigplan Notices, Vol. 8, No. 8 (1973), pp. 12-26.

NAYLOR, T. H., "Decision Support Systems, or Whatever Happened to M.I.S.?", TIMS Interfaces, Vol. 12, No. 4 (August 1982), pp. 92-94.

Nelson, T. H., "Getting It Out of Our System", in G. Slechter (Ed.), Information Retrieval: A Critical Review, Thompson Books, Washington D.C., 1967, pp. 191-210. 
Ness, D. N., "Decision Support Systems: Theory of Design", Proceedings of the Joint Wharton/ONR Conference on Interactive Information and Decision Support Systems, Philadelphia, PA, 1975.

Nowotny, O. H., "Verrechnungspreise Zwischen Verbundenen Unternehmen", Pharma Industrie, Vol. 49, No. 4 (1987), pp. 339-346 (in German).

Olle, T. W., J. Hagelstein, I. G. Macdonald, C. Rolland, H. G. Sol, F.J.M. van Assche AND A. A. VerriJn-STuart, Information Systems Methodologies: A Framework for Understanding, Addison-Wesley, Workingham, England, 1988.

Olson, M. H. And J. A. Turner, "Rethinking Office Automation", in L. Gallegos, R. Welke and J. Wetherbe (Eds.), Proceedings of the $6^{\text {th }}$ International Conference on Information Systems, Indianapolis, December 1985, pp. 259269.

OUWEHAND, J. J., "Roosteren via computer geeft bedrijven en personeel meer opties", Tijdschrift Nederlandse Chemische Industrie, Vol. '89, No. 10 (May 1989), pp. 10-11 (in Dutch)

PANKo, R. R., "38 Offices: Analyzing Needs in Individual Offices", $A C M$ Transactions on Office Information Systems, Vol. 2, No. 3 (July 1984), pp. 226-234.

Panko, R. R. and R. H. Sprague, JR., "Implementing Office Systems Requires a New DP Outlook", Data Management, Vol. 22, No. 11 (November 1984), pp. $40-42$.

Parsons, G. L., "Information Technology: A New Competitive Weapon", Sloan Management Review, Vol. 25, No. 1 (1983), pp. 3-14.

Payne, J. W., "Task Complexity and Contingent Processing in Decision Making: An Information Search and Protocol Analysis", Organizational Behavior and Human Performance, Vol. 16, No. 2 (August 1976), pp. 366-387.

Pernici, B. And A. A. Verrijn-Stuart (Eds.), Office Information Systems: The Design Process, Elsevier Science Publishers (North-Holland), Amsterdam, 1989.

Petri, C. A., "Kommunikation mit Automaten", in E. Peschi and H. Unger (Eds.), Schriften de Westfälischen Institutes für Instrumentelle Mathematik an der Universität Bonn, No. 2 (1962).

Pitz, G. F., "Human Engineering of Decision Aids", in T. Engländer (Ed.), Proceedings of the $8^{\text {th }}$ Research Conference on Subjective Probability, Utility and Decision-making, Budapest, 1981.

Plasschaert, S. R. F., Transfer Pricing and Multinational Corporations: An Overview of Concepts, Mechanisms and Regulations, Saxon House Westmead, England, 1979.

Poppel, H. C., "Who Needs the Office of the Future?", Harvard Business Review, Vol. 60, No. 6 (November/December 1982), pp. 146-155. Rematch, VUMAS Users Manual, Rematch B.V., Amsterdam, The Netherlands,
1987.
Richter, G., P. Humphreys, K. Voss, D. Berkeley, H. Genrich, M. Domke, H. Griebler and A. Wisudha, Generic Office Frame of Reference (GOFOR), Gesellschaft für Mathematik und Datenverarbeitung, Sankt Augustin, West Germany, June 1987.

Rowe, L. A., M. Davis, E. Messinger, C. Meyer, C. Spirakis and A. Tuan, "A Browser for Directed Graphs", Software - Practice and Experience, Vol. 17, No. 1 (January 1987), pp. 61-76.

Roy, B., Méthodologie Multicrité d'Aide à la Décision, Economica, Paris, France, 1985 (in French).

Sage, A. P., B. Caling and A. Lagomasino, "Methodologies for Determination of Information Requirements for Decision Support", Large Scale Systems, Vol. 5, No. 2 (1983), pp. 131-167.

ShaKespeare, William, "A Midsummer-Night's Dream", see for example The Complete Works of William Shakespeare, Abbey Library, London, England, 1974, p. 192.

Shakespeare, William, "Macbeth", see for example The Complete Works of William Shakespeare, Abbey Library, London, England, 1974, p. 826.

Simon, H. A., The New Science of Management Decision, Harper \& Row, New York, NY, 1960.

Simon, H. A., "The Structure of Ill-Structured Problems", Artificial Intelligence, Vol. 4 (1973a), pp. 181-202

Simon, H. A., "Applying Information Technology to Organizational Design", Public Administration Review, Vol. 33, No. 3 (May/June 1973b), pp. 268278.

Simon, H. A., The New Science of Management Decision (revised edition), Prentice-Hall, Englewood Cliffs, 1977.

Simon, H. A., The Sciences of the Artificial ( $2^{\text {nd }}$ edition), MIT Press, Cambridge, MA, 1981.

Simon, H.A., G.B. Dantzig, R. Hogarth, C.R. Plott, H. Raiffa, T.C. Schelling, K. A. Shepsle, R. Thaler, A. Tversky and S. Winter, "Decision Making and Problem Solving", TIMS Interfaces, Vol. 17, No. 5 (September/October 1987), pp. 11-31.

Singer, G. AND Y. Berger, "Rosters for Irregular Hours", in M. Wallace (Ed.), Improvements in Shiftwork, Group Publications, David Syme \& Co. Limited, Melbourne, Australia, 1986, pp. 17-18.

Smith, J. B. AND S. F. WeIss, "Hypertext", Communications of the ACM, Vol. 31, No. 7 (July 1988), pp. 816-819.

Sol, H. G., "Simula(tion) in the Analysis and Design of Information Systems", in M. H. Hamza (Ed.), Simulation 77, Acta Press, Anaheim, Norway, 1977.

Sol, H. G., Simulation in Information Systems Development, Doctoral dissertation, University of Groningen, The Netherlands, 1982. 
Sol, H. G., "DSS: Buzzword or OR Challenge?", European Journal of Operational Research, Vol. 22, No. 1 (1985), pp. 1-8.

SoL, H. G., "Paradoxes around DSS", in C. W. Holsapple and A. B. Whinston (Eds.), Decision Support Systems: Theory and Application, Springer-Verlag, Berlin, 1987, pp. 3-18.

Sol, H. G., "Information Systems Development: A Problem Solving Approach", Proceedings of the Symposion on Systems Analysis and Design, Atlanta, November 1988

Sol, H. G. AND M. B. M. VAN DER VEN, "Integrating GDSS in the Organization The Case of a GDSS for International Transfer Pricing", in R. M. Lee, A. M. McCosh and P. Migliarese (Eds.), Organizational Decision Support Systems, Elsevier Science Publishers (North-Holland), Amsterdam, 1988, pp. 15-30.

Sol, H. G. AND M. B. M. VAN DER VEN, "A Group Decision Support System for International Transfer Pricing Decisions in the Pharmaceutical Industry", in M. G. Singh, K. S. Hindi and D. Salassa (Eds.), Managerial Decision Support Systems, Elsevier Science Publishers (North-Holland), Amsterdam, 1988, pp. 81-94.

SøLVBERG, A., "The Use of Models and Associated Software in the Design of Wicked Systems", in E. Grochla and S. Szyperski (Eds.), Information Systems and Organizational Structure, De Gruyter, Berlin, 1975.

Sprague, R. H., "A Framework for the Development of Decision Support Systems", Management Information Systems Quarterly, Vol. 4, No. 4 (December 1980), pp. 1-26.

Sprague, R. H., "DSS in Context", in E.R. McLean and H.G. Sol (Eds.), Decision Support Systems: A Decade in Perspective, Elsevier Science Publishers (North-Holland), Amsterdam, 1986, pp. 19-24.

Sprague, R. H. ANd H. J. Watson (Eds.), Decision Support Systems: Putting Theory into Practice, Prentice-Hall, Englewood Cliffs, NJ, 1986.

StaBell, C. B., "A Decision-Oriented Approach to Building Decision Support Systems", in J. Bennett (Ed.), Building Decision Support Systems, AddisonWesley, Reading, MA, 1983

Stoica, M. AND E. Scarlat, "Fuzzy Concepts in the Control of Production Systems", in Proceedings of the $3^{\text {rd }}$ International Congress of Cybernetics and Systems, Bucharest, Rumania, Agust 1975.

Sugiyama, K., S. Tagawa And M. Toda, "Methods for Visual Understanding of Hierarchical System Structures", IEEE Transactions on Systems, Man, and Cybernetics, Vol. 11, No. 2 (February 1981), pp. 109-125.

Thierauf, R. J., Decision Support Systems for Effective Planning and Control: A Case Study Approach, Prentice-Hall, Englewood Cliffs, NJ, 1982.

TodD, P. AND I. BEnBasat, "Process Tracing Methods in Decision Support Systems Research: Exploring the Black Box", Management Information Systems Quarterly, Vol. 11, No. 4 (December 1987), pp. 493-512.
TsichritzIs, D. C., "A Form Manipulation System", in Proceedings of the NYU Symposium on Automated Office Systems, New York University, New York, NY, 1979.

TsichritzIs, D. C., "Form Management", Communications of the ACM, Vol. 25, No. 7 (July 1982), pp. 453-478.

Tsichritzis, D. C., E. Fiume, S. GibBs and O. Nierstrasz, "KNOs: KNowledge Acquisition, Dissemination and Manipulation Objects", ACM Transactions on Office Information Systems, Vol. 5 (1987), pp. 96-112.

Turban, E. AND P. R. WatKins, "Integrating Expert Systems and Decision Support Systems", Management Information Systems Quarterly, Vol. 10, No. 2 (June 1986), pp. $121-136$

TVErsky, A. AND D. Kahneman, "Judgement under Uncertainty: Heuristics and Biases", Science, Vol. 185, No. 4157 (September 1974), pp. 1124-1131.

VAN DER Ven, M. B. M., A Group Decision Support System for International Transfer Pricing in the Pharmaceutical Industry, Doctoral dissertation, Delft University of Technology, The Netherlands, 1989.

VAn DE Ven, A. H., "Problem Solving, Planning and Innovation, Part I. Test of the Program Planning Model", Human Relations, Vol. 33, No. 10 (October 1980), pp. 711-740.

VAN Gundy, A. B., Techniques of Structured Problem Solving, Van Nostrand Reinhold, New York, NY, 1981.

VAN Sch.4IK, F. D. J., Effectiveness of Decision Support Systems, Doctoral dissertation, Delft University of Technology, The Netherlands, 1988

AN SCHAIK, F. D. J. AND H. G. Sol, "Effectiveness of Environments for Supporting Decision Making: The Case of Equation Models versus Proces Models", in Stimulerende Informatica, Proceedings of the NGI-SION Symposium 4, Utrecht, The Netherlands, April 1986, pp. 89-94.

VAN WEELDEREN, J. A. AND H. G. Sol, "The Xpection-project: Development of an Expert Support System for the Maintenance of Boiler Components Operating in the Creep Range", in Proceedings of the Symposium on Expert Systems Application to Power Systems, Royal Institute of Technology, StockholmHelsinki, September 1988.

Verlage, H. C., Transfer Pricing for Multinational Enterprises, Doctoral dissertation, Rotterdam University Press, Rotterdam, the Netherlands, 1975

Verrijn-Stuart, A. A., "The Past and Future of Information Systems", in P. A Samet (Ed.), EURO IFIP 79, North-Holland Publishing Company, Amsterdam, 1979, pp. 153-161.

VerRIJN-Stuart, A. A., "Themes and Trends in Information Systems: TC8, 1975 1985", The Computer Journal, Vol. 30, No. 2 (1987b), pp. 97-109.

VERRIJn-Stuart, A. A., The Information System in the Broader Sense, Report No. 89-13, Department of Computer Science, Leiden University, The Netherlands, 1989. 


\section{INDEX AND GLOSSARY}

Verrijn-Stuart, A. A. And P. W. G. Bots, Diversity and Uniformity in Office Models, Report No. 87-25, Department of Computer Science, Leiden University, The Netherlands, 1987.

VoN Grebmer, K., "International Transfer Pricing in the Pharmaceutical Industry", Intertax, Vol. 4, No. 5 (1987), pp. 92-99.

Wallace, M., "The Length of Shift and the Measurement of Effort", in M. Wallace (Ed.), Improvements in Shiftwork, Group Publications, David Syme \& Co. Limited, Melbourne, Australia, 1986, pp. 5-8.

Weick, K. E., The Social Psychology of Organizing, Addison-Wesley, Reading MA, 1979.

WildE, OscaR, Act III of "Lady Windermere's Fan", see for example I. Murray (Ed.), Oscar Wilde, Oxford University Press, Oxford, England, 1989, p. 373.

Woo, C. C. AND F. H. Lochovsky, "Supporting Distributed Office Problem Solving in Organizations", ACM Transactions on Office Information Systems, Vol. 4, No. 3 (July 1986a), pp. 185-204.

Woo, C. C. AND F. H. Lochovsky, "Integrating Procedure-Automation and Problem-Solving Approaches to Supporting Office Work", in G. Bracchi and D. C. Tsichritzis (Eds.), Office Systems: Methods and Tools, preprints of the IFIP WG8.4 Working Conference on Methods and Tools for Office Systems, Pisa, Italy, October 1986b, pp. 15-30.

Young, L. F., "A Corporate Strategy for Decision Support Systems", Journal of Information Systems Management, Vol. 1, No. 1 (Winter 1984).

Yourdon, E. And L.L. Constantine, Structured Design, Prentice-Hall, Englewood Cliffs, NJ, 1979

Zeleny, M., Multiple Criteria Decision Making, McGraw-Hill, New York, N.Y., 1982.

Zisman, M., "Use of Production Systems for Modeling Asynchronous, Concurren Processes", in D. A. Waterman and F. Hayes-Roth (Eds.), Pattern Directed Inference Systems, Academic Press, New York, NY, 1978, pp. 53-68.

ZLOOF, M. M., "QBE/OBE: A Language for Office and Business Automation", IEEE Computer, Vol. 14, No. 5 (May 1981), pp. 13-22.

Zloof, M. M. AND S. P. DE Jong, "The System for Business Automation (SBA) Programming Language", Communications of the ACM, Vol. 20, No 6 (June 1977), pp. 324-343.
What does Crustimoney Proseedcake mean? Very Little Brain, and long words Bother me. Winnie-the-Pooh
¿ see task symbols; "explosive" $\lambda$ see flow relation operato

$\lambda$ see label

\| see concurrency

a priori unstructured 17

a priori structured 17,20

a posteriori structured 17,20 abort 46

Ackoff, R.L. 13, 17

action 31

ACTION LOGGER 77,85

action method 57

Active 42

actual remittance 160

$\mathcal{A}_{D} 42$

adaptive design

adaptivity 171

affected part 49 ,

see also information

agenda 42,45

agenda mechanism 71

aggregation level $57,59,15$

Aiello, L. 7

Algol 127

alias 77

Alter, S.L. 4

ALTERNATIVE 141

alternative courses of action 13

Amarel, S. 14

artefact 35

ARTICLE 138

Asai, K. 5

$\mathcal{A}_{T} \quad 45$ attribute 31

ATTRIBUTE MONITOR 77,85

AUTHORIZER 140

automatic pilot 116

AW-V 94

ACKGROUND INFORMATION ITEM 141

bandwagon effect 3

BANK 180

Barber, G. 7

Barr, A. 5, 43

Belew, R.K. 4

Ben-Bassat, M. 5

Bennett, J.L. 4

Berger, Y. 91

Bernal, M. 7

Beta 31

Betz, F. 13

BLOCK 117

blueprint 26

BOILER 178

Bonczek, R.H. 4, 5

Borland 86, 129

Bosman, A. 2, 4, 5, 17

Bots, P.W.G. $8,10,216$ bounded rationality 14

Bracchi, G. 6

Brown, J.S. 2

Brussaard, B.K. 10

BUDGET 141

Bush, V. 1

C 31

candidate list 120

Capra, Fritjof 51 
Carpano, M.J. 79

Carphen, Caril vii

case study 12

Cashman, P. 38

CELL MATRIX 115

Chandrasekaran, B. 5

Chang, M.G. 4

choice 19

CLA see common labor agreement

class browser 75

CLASS HIERARCHY BROWSER 85

common labor agreement 92

COMPANY 137,175

competitive advantage 2

conceptual model 30

of shiftwork planning 98

of transfer pricing 145

of VUMAS 181, 183

conceptualization $17,31,34,54$

support 58

concurrency 48

confusion 48

consistency $30,53,63$

Constantine, L.L. 37

CONTRACT 176

COORDINATING DECISION 84

see also decision

coordinating mechanism 22-23

coordination 12

coordination problem 22

detection 36

correspondence 30,53

cost transfer 134

COUNTRY 138

Cox, B. 32

crew 89

crew roster 95

CREWING SYSTEM 94

crewing system 89

examples 90,100

selection 106

Croft, W.B. 7, 45 current account 134

Daft, R.L. 16

Dahl, O.J. 8

Dawson, K. 91

De Jong, S.P. 6

DECISION 84

decision 18

automated 67,79

coordinating 43

formal definition 40

leaf 43

made 46

DECISION BROWSER 79

decision calculus 4

decision making 19 multi-criteria 167

decision room 168

decision rule 42

decision structure 40-41, 49

condensed 41, 126

expanded 41

graphical representation 41

decision support system 3

definitions 4

movement 3

decision symbols 47

demarcation 57

demarcation rule 10,182

DeMarco, T. 37

demon 42, 50

DeSanctis, G. 168

design 19,220

DICTIONARY INSPECTOR 85

Digitalk 83

Dijkstra, E.W. 37

direct supervision 22

dividend 134

division of labor 22, 182

document base 85

DOCUMENTATION BROWSER 74

Donovan, J.J. 4

Doyle, A.C. 29
$\mathcal{D}_{\mathrm{P}}$ see decision structure

DSS see decision support system

Einhorn, H.J. 18

Elam, J.J. 4

Ellis, C.A. 6, 7, 9

emergency loan 180

empirical model 30

of shiftwork planning 100-103

of transfer pricing 144-146

of VUMAS 184-188

encapsulation 31

environment

of an IS/RS-combination 10

to support problem solving

see problem solving support environment

equivocality 16

error of the third kind 18

Europhar 136, 144

evaluation criteria 16

EXCEPTION 99

executive feel 168

expert systems 5

FACILITATOR 165

Featheringhouse, F.R. 13, 18

Feigenbaum, E.A. 5, 43

FIELD 114

Finance department's problem 196

financial impact 160,165

Flores, F. 7

flow 47

flow diagram 47

flow relation 45

operator 46

flowchart 36

FORM EDITOR 75

$\mathcal{F}_{\mathrm{P}}$ see flow relation

FUNCTION 95

function group 103

fund transfer alternatives 133-134

fundamental assumptions 21

Galbraith, J.R. 16
Gallupe, B. 168

Garp, T.S. 13

GDSS see group decision support system

General Industrial Employer's

Association 92

generate-and-test algorithm 18, 211

Gibbs, S. 7

Ginzberg, M.J. 4

Gluss, B. 5

Goldberg, A. 8, 32, 75, 81, 83, 85

Gorry, G.A. 3

GOTO-less programming $36-37$

GOVERNMENT 180

graph layout algorithm 79

Gray, P. 171

group decision support system 165

group problem solving 133

Guépin, J.P. 241

Hackathorn, R.D. 17

Hämmäinen, H. 7

hard core 26

Hewitt, C.E. 7

Hirschheim, R.A. 7

Hogarth, R.M. 18

Holbæk-Hanssen, E. 8

Holloway, C.A. 18

Holmes, Sherlock 29

Holt, A.W. 38

$\mathcal{H}_{\mathrm{P}}$ see task hierarchy

Huber, G.P. 1, 2, 17, 168

Hypertext 1

icon 55

ICON EDITOR 75

immediate precursors 46

immediate successors 46

implementation $20,30,70$

inconvenience 101

independent task 43

INDIVIDUAL ROSTER 96

influencing part 49 ,

see also information 
information 25 affected 38

available to VUMAS players 186

formal definition 40

influencing 38

item 43

paradigm 10

society 1

technology 1,40

INFORMATION BROWSER 80

information system 1, 9-11

design methodologies 36

support decision processes 6

inheritance 31,136

initial items 46, 71

inspector 81

INSTALLATION 177

intelligence 19

intra-company loan 134

Irving, John 51

IS see information system

IS/RS-combination 10

Jacob, J. 91

Jarke, M. 9, 168

Joyner, R. 17, 167

Kahneman, D. 18

Kappel, G. 7

Keen, P.G.W. 2-4, 6, 9, 133

kernel support objects $73,82,83$

Knuth, D.E. 37,127

Kung, C.H. 40,50

label 46

LABOR CONDITION 94

examples 102

Lafferty, R.A. 249

Lakatos, I. 12

Langefors, B. 36

leaf decision see decision

Lee, R.M. 4, 40

Lefkowitz, L.S. 7, 45

Lengel, R.H. 16

liquidity 133
Lisp 31, 37

Little, J.D.C. 4, 18, 119

LOAN 180

LOCAL AUTHORITY 139

Lochovsky, F.H. 7-9, 36, 45, 46

Lockemann, P.C. 31

Lootsma, F.A. 170

Lundeberg, M. 37

Lundt, O.E. 108

macro-perspective 10

Madnick, S.E. 4

Madsen, O.L. 32

Maes, R. 17

management game 171

manning requirements 101

March, J.G. 14, 18

Marketing \& Sales department's problem 195

maximal remittance 157

McDaniel, R.R. 2, 17

McFarlan, F.W. 2

McGettrick, A.D. 188

McLean, E.R. 4

MEDIATOR 9

megatrends 1

memory 42

meso-perspective 10

Meyer, B. 8

micro-perspective 10

Milne, A.A. 233

Miner, F.C. 18

Mintzberg, H. 7, 15, 17

MIS see management information system

Mitroff, I.I. 13, 17, 18

model browser 76

model-oriented 15

Montaigne, Michel de 221

Moon, D.A. 32

Moore, J.H. 4

mutual adjustment 22

Naffah, N. 9
Nassi, I. 37

natural language

Naylor, T.H. 4

negative selection 151,154

Nelson, T.H. 1

Ness, D.N. 6

network 81

Nierstrasz, O. 31

non-procedural languages 37

non-programmed 14

NOTIFICATION OF ABSENCE 99

NOTIF ICATION OF INDIVIDUAL PREFERENCE 99

Nowotny, O.H. 136

Nutt, G. 6

object class 31

"artificial" 35

"directly visible" 33

notation 32

refinement/redefinition 35

object instance 31

notation 83

object-oriented modeling 8,31

office information system 6

office models 7

OIS see office information system

Olle, T.W. 45

Olson, M.H. 9

OMEGA 7

open systems 7

operations 16

optimizing algorithms 116

organizational decision making 2

organizational problem solving 171

Ouwehand, J.J. 91

Panko, R.R. 8

PAPS 8

PARENT COMPANY 137

Parsons, G.L. 2

partial solution 151

PARTICIPANT 140

Pascal 129

Passive 42 patching 110,132

Payne, J.W. 36

performance

human 2

organizational 26,220

Pernici, B. 6, 7, 38

PERSONNEL MANAGER 97

Personnel department's problem 192 personnel satisfaction indicator 185 Petri, C.A. 38

Petri net 37

Pitz, G.F. 18

Plasschaert, S.R.F. 136

Poppel, H.C. 7

precedence analysis 36

precedence graph 36

preference ordering 158,161

problem 13

problem situation 30

problem solving 13

activities 29

individual 89

phases 19

process 29

programme see programme

stages 29

strategy 17

support environment 51

functional requirements $73-82$

global architecture 73

problem structure 17

procedure-oriented 15

process control systems 5

PRODUCT 177, 184

PRODUCT MARKET 179

PRODUCTION DEPARTMENT 94

Production department's problem 191

production hours 89

PRODUCTION UNIT 93

program 16 
programme 16

representation 17,39

programmed 14

Prolog 37

PROMPTER 80

protective belt 26

PSI see personnel satisfaction indicator

PSSE see problem solving support environment

Purchase department's problem 188 rationality 13

RAW MATERIAL 176

real system 10

real-life 14,92

recursion principle 10

regulations 139

relations 16

Rematch 171

RESEARCH INSTITUTE 180

research programme 12,26

research question $11-12,25$

review 20

Richter, G. 38

Robson, D. 8, 32, 83

ROPAQ 116

ROSTER PERIOD 95

roster worksheet 113

Rowe, L.A. 79

Roy, B. 170

royalty 134

RS see real system

Sage, A.P. 14

sample data 60

Scarlat, E. 5

Scott Morton, M.S. 3

sequencing 48

Shakespeare, William ix, 217

Shapira, Z. 18

share capital 134

SHIFT 94

shiftwork 89
Shneiderman, B. 37

signal 48

Simon, H.A. 2, 13, 14, 18, 19, 150

Singer, G. 91

Smalltalk 31, 83

Smith, J.B. 1

software engineering

methodologies 36

Sol, H.G. 2-6, 8-10, 12, 15, 17, 25, $29,51,133,168,216$

solution finding $18,30,64$ support 70

S $\phi$ lvberg, A. 14, 40, 50

specific DSS 4, 188

specification 18, 34, 59 support 62

Sprague, R.H. 2, 4-6, 8, 9

Stabell, C.B. 26

standardization 22

STATISTICAL ANALYZER 77

Stohr, E.A. 4

Stoica, M. 5

Stroustrup, B. 32

structured programming 36

structuredness 14-18, 49, 64

subclass 136

subproblem 21

SUBSIDIARY COMPANY 137

subtask 21, 43

Sugiyama, K. 79

SUPPORT BROWSER 81

support component 40

setting priorities 111

synchronization 48

system 51

ABLE 113

tallies 96

Tanaka, H. 5

Tas, P.A. 10

TASK 84

task 20, 24

execution 67 formal definition 43

initiated 46

structuredness 44, 49

Type I/Type II 8

task analysis 36

TASK BROWSER 77

task hierarchy 45

task structure 21, 49

formal definition 44

graphical representation 48

task symbols 44

explosive 47

TEAM 93

team 89

TEAM ROSTER 95

terminate 46

Thierauf, R.J. 4

THING 83

thresholds 122

time savings 112,131

$\mathcal{T}_{\mathrm{P}}$ see task structure

transaction processing 13

transfer price 134

transient targets 158

Tsichritzis, D.C. 2,7

Tunstall, K. 17, 167

Turban, E. 6

Turner, J.A. 9

Tverski, A. 18

uncertainty 16

understanding 220

UNION 94,179

validation 35

Van der Ven, M.B.M. 133-136, 144,

$146,152,156,160,168,170-17$

Van de Ven, A.H. 17

Van Gundy, A.B. 17

Van Schaik, F.D.J. 17, 53, 173, 180, 191, 215, 216

Van Weelderen, J.A. 6

variables 16

Verbraeck, A. 132
Verlage, H.C. 136

Verrijn-Stuart, A.A. 1, 7, 8, 38

vocabulary 31

Vogel, D.R. 171

Von Grebmer, K. 136

VUMAS 171

Waiting 42

Wallace, M. 91

WAREHOUSE 179

Watkins, P.R. 6

Watson, H.J. 4

Weick, K.E. 16

weight function 38

Weiss, S.F. 1

Wilde, Oscar 133

Woo, C.C. 8,9

WORK FORCE 178

WORKER 93

working hours 89,10

instruments to decrease 90-91

workplace 9,12

ORKSHEET 115

WORLD 83

yardstick 220

Young, L.F. 15

Yourdon, E. 37

Zeleny, M. 170

Zisman, M. 7

Zloof, M.M. 6, 7 


\section{SAMENVATTING}

\section{PROBLEEMSTELLING}

Het vakgebied Informatiesystemen wordt veelal ingedeeld naar de aard van verschillende toepassingen, zoals bijvoorbeeld geautomatiseerde gegevensverwerking (EDP) voor administratieve taken, management-informatiesystemen (MIS) voor reguliere rapportage, beslissingsondersteunende systemen (DSS) voor het oplossen van slechtgestruktureerde problemen, kantoorinformatiesystemen (OIS) voor multi-media omgevingen, en expertsystemen (ES) ter vervanging van een menselijke beslisser. Indien men echter de ontwikkelingen in een breder kader beziet kan men de volgende drie "megatrends" waarnemen:

1. informatietechnologie wordt toegepast voor het verkrijgen van een voorsprong op concurrerende organisaties

. het nemen van beslissingen wordt de primaire aktiviteit binnen organisaties

3. informatietechnologie wordt specifiek aangewend ter verbetering van menselijke prestaties.

Dit streven naar een tactische voorsprong, waarbij beslissen de primaire aktiviteit is, en waarbij de mens en niet de machine centraal staat is bepalend voor het ontwerp van toekomstige informatiesystemen.

Informatiesystemen zijn geen doel op zich; zij vormen een belangrijk onderdeel van organisaties, maar het feitelijke doel is prestatieverbetering van die organisaties. Organisaties, en daarmee hun informatiesystemen, kunnen worden beschouwd op drie niveaus:

1. op het micro-niveau kijkt men naar personen, hun specifieke taken, en de wijze waarop informatietechnologie de uitvoering van die taken kan ondersteunen teneinde de individuele prestatie te verhogen;

2. op het meso-niveau wordt gekeken naar de coördinatie tussen individuen op hun werkplek, en die tussen werkplekken onderling, waarbij gestreefd word naar het verbeteren van de organisatiestruktuur, zowel qua besluitvorming als qua informatieuitwisseling;

3. op het macro-niveau beschouwt men een aantal organisaties tegelijk, waarbij de nadruk ligt op het verhogen van het gemeenschappelijke prestatieniveau, danwel het verbeteren van de positie van de eigen organisatie ten opzichte van die van anderen. 
Het onderzoek waarvan dit proefschrift verslag doet richt zich op de eerste twee niveaus. Uitgangspunt van dit onderzoek is de stelling dat het nemen van beslissingen de primaire aktiviteit binnen organisaties vormt, en dat informatietechnologie moet worden aangewend ter ondersteuning van beslissingsprocessen van individuen en groepen. Verder wordt aangenomen dat menselijke beslissers beperkt rationeel handelen. Doel van het onderzoek is na te gaan:

1. in hoeverre concepten uit de theorie rond probleem-oplossen en beslissen kunnen dienen als uitgangspunt voor het zodanig toepassen van informatietechnologie binnen organisaties dat een verbetering van zowel de individuele prestaties als de onderlinge coördinatie wordt gerealiseerd;

2. welke eisen een dergelijke probleemgerichte aanpak stelt met betrekking tot technische ondersteuning, en in hoeverre aan deze eisen kan worden voldaan.

Teneinde een antwoord op deze vragen te vinden wordt een research programma uitgevoerd, waarvan de harde kern bestaat uit de vooronderstelling dat de prestatie van een organisatie direkt gerelateerd is aan het probleemoplossend vermogen en de onderlinge coördinatie van de beperkt rationeel handelende individuen in die organisatie. De te toetsen theorie bestaat uit een techniek voor het formuleren en struktureren van problemen, en de claim dat de probleembeschrijvingen die met deze techniek kunnen worden gemaakt een "blauwdruk" vormen voor probleem-specifieke informatiesystemen. Deze theorie word getoetst aan twee criteria: haalbaarheid door te bepalen wat voor computerondersteuning vereist is om de genoemde techniek toe te passen en na te gaan of deze ondersteuning realiseerbaar is, en bruikbaarheid door de techniek toe te passen op een drietal probleemsituaties en na te gaan of hij inderdaad resulteert in bruikbare specificaties.

\section{FORMULEREN EN STRUKTUREREN VAN PROBLEMEN}

Een probleem wordt gedefinieerd als een situatie waarin een beslisser kan kiezen uit een aantal mogelijke alternatieven, de gemaakte keuze aanzienlijke gevolgen kan hebben, en de beslisser enige twijfel heeft met betrekking tot het kiezen van én bepaald alternatief. Een beslissing wordt gezien als een keuze tussen een aantal alternatieven tijdens het oplossen van een probleem. Het oplossen van een probleem wordt een taak genoemd. Omgekeerd wordt alles wat normaliter als een taak wordt beschouwd gezien als probleem-oplossen. Als regel kan een probleem worden opgedeeld in een aantal deelproblemen. Het oplossen van een deelprobleem vormt dan een deeltaak. Tenzij expliciet anders vermeld wordt met de term "beslissing" de keuze zelf, en niet het maken van die keuze of de gemaakte keuze bedoeld. Bijgevolg refereert "taak" aan een (statische) verzameling van deeltaken en beslissingen en hun opeenvolging in de tijd, en niet aan het (dynamische) proces dat plaatsvindt wanneer een taak wordt uitgevoerd.

Bij het beschrijven van een probleem dient men onderscheid te maken tussen probleemstruktuur en taakstruktuur. Met probleemstruktuur wordt gedoeld op het samenstelsel van variabelen, daarop gedefinieerde operaties, relaties tussen variabelen, en evaluatiecriteria in termen waarvan een probleemsituatie volledig kan worden gedefinieerd. Onder taakstruktuur wordt de beschrijving van een "stappenplan" om tot een oplossing voor een probleem te komen verstaan. In zo'n stappenplan wordt uiteraard gerefereerd aan elementen van de probleemstruktuur.

Het oplossen van een probleem wordt gezien als bestaande uit vier fasen conceptualisatie, specificatie, het zoeken naar een oplossing, en implementatie. Conceptualisatie omvat het identificeren van de klassen van objekten die een rol spelen in de probleemsituatie, en het beschrijven van hun eigenschappen, mogelijke akties en onderlinge relaties. De resulterende verzameling objektdefinities wordt aangeduid als een conceptueel model. Zo'n model vormt het definities wordt aangeduid als een conceptueel model. Zo'n model vormt het beschreven door instanties van objektklassen te creëren en aan hun attributen de juiste beginwaarden toe te kennen. Indien het conceptuele model meer details blijkt te beschrijven dan voor het vinden van een oplossing noodzakelijk wordt geacht kunnen "artefacten" worden geïntroduceerd: objectklassen die de eigenschappen van een aantal klassen uit het conceptuele model in zich verenigen Door instanties te specificeren ontstaat een empirisch model. In feite beschrijft zo'n model de probleemstruktuur voor zover dat mogelijk is. Een empirisch Zorkelijkheid te vertonen. Als regel zullen de conceptualisatie- en specificatie-fase een aantal malen worden doorlopen voordat voldoende overeenstemming is bereikt. Het zoeken naar een oplossing komt neer op het ontwerpen van een taakstruktuur die beschrijft hoe het probleem kan worden opgelost. De voor- en nadelen van verschillende taakstrukturen kunnen worden ingeschat door ze te testen aan de hand van het empirisch model. Implementatie komt uiteindelijk neer op het daadwerkelijk uitvoeren van het stappenplan dat door de gekozen taakstruktuur wordt beschreven.

Een taakstruktuur voor een probleem bestaat uit twee componenten: een taakhierarchie en een stroomrelatie. De taakhierarchie ontstaat doordat een taak - het oplossen van het probleem - recursief wordt gedefinieerd in termen van beslissingen en deeltaken. De stroomrelatie bepaalt de volgorde waarin taken en beslissingen moeten worden uitgevoerd. Zolang een taak subtaken heeft zijn de beslissingen binnen die taak coördinerende beslissingen in de zin dat zij bepalen welke deeltaken moeten worden uitgevoerd, en in welke volgorde. Een taak die niet verder wordt gesplitst in deeltaken heet een bladtaak. Van een bladtaak wordt aangegeven welke beslissingen bij het uitvoeren daarvan moeten worden genomen. Van elk van deze bladbeslissingen wordt vastgesteld welke informatie 
van invloed is op de te maken keuze, en welke informatie door het maken van die keuze wordt beïnvloed. Deze informatie moet kunnen worden aangeduid in termen van het conceptuele model. Er kan nu een precedentierelatie worden gedefinieerd aan de hand waarvan kan worden nagegaan in hoeverre de beslissingen in een taakstruktuur samenhangen. Deze samenhang wordt de beslissingsstruktuur van een taak genoemd. Het gehele proces van het ontwerpen van een taakstruktuur en het bepalen en analyseren van beslissingsstrukturen wordt taakanalyse genoemd.

Eenmaal vastgesteld kan een taakstruktuur dienen als uitgangspunt bij het zoeken naar mogelijkheden voor computerondersteuning. Per beslissing kan worden nagegaan in welke vorm de beïnloedende informatie gepresenteerd kan worden, en in hoeverre de beslisser gebaat is bij analytische hulpmiddelen. Daarnaast kan een schatting worden gemaakt van mogelijke efficiëntieverbetering door per taak na te gaan hoe vaak deze zal worden uitgevoerd, hoeveel tijd voor die uitvoering nodig is, en in welke mate die tijd door middel van computerondersteuning zou kunnen worden bekort. Op grond van dergelijke inschattingen kunnen prioriteiten worden gesteld ten aanzien van de te ontwikkelen software.

\section{HAALBAARHEID}

De behoefte aan computerondersteuning tijdens het toepassen van de zojuist beschreven techniek voor het formuleren en struktureren van problemen is bepaald door taakanalyse toe te passen op de taak "Los probleem op". Zo zij de fasen conceptualisatie, specificatie, zoeken naar een oplossing, en implementatie opgevat als deeltaken van "Los probleem op", en is elk van deze vier afzonderlijk verder opgesplitst. Uiteindelijk zijn elf bladtaken geanalyseerd. De resulterende taakstruktuur vormt niet alleen een gedetailleerd stappenplan voor probleemformulering en -strukturering, maar ook een blauwdruk voor een "omgeving" ter ondersteuning van het oplossen van problemen. Deze omgeving "omgeving" ter ondersten

1. het definiëren van objektklassen en bijbehorende grafische representaties;

2. het samenstellen, analyseren en visualiseren van conceptuele modellen;

3 . het maken en testen van empirische modellen;

4. het ontwerpen en analyseren van taakstrukturen en beslissingsstrukturen;

5. het uitproberen van taakstrukturen aan de hand van een empirisch model

Door een prototype in Smalltalk op een PC-AT te ontwikkelen is aangetoond dat deze faciliteiten voor relatief kleine probleemsituaties inderdaad te integreren zijn. De bij dit proefschrift geleverde software ondersteunt taakanalyse van grotere problemen, maar biedt nog geen faciliteiten voor het dynamisch modelleren van probleemsituaties.

\section{BRUIKBAARHEID}

Om de bruikbaarheid van de zoëven beschreven techniek voor het formuleren en struktureren van problemen te toetsen is een drietal probleemsituaties geanalyseerd. Bij de probleemkeuze is met name gekeken naar het aantal beslissers, de complexiteit van de probleemstruktuur, en in hoeverre er al een taakstruktuur voor het probleem bestond. Doel van de eerste casus is na te gaan of een taakstruktuur daadwerkelijk geschikt is voor het gedetailleerd beschrijven van een beslissingsproces. Aan de hand van de tweede casus kan worden nagegaan in hoeverre de voorgestelde techniek ook geschikt is voor probleemsituaties waarbij meerdere beslissers zijn betrokken. Bij de derde casus wordt dit opnieuw getoetst en wordt bovendien gekeken of het mogelijk is om uitgaande van een beslissingsstruktuur te komen tot een taakstruktuur. Daarnaast word met elk van de drie studies getoetst of een taakstruktuur een goed uitgangspunt vormt voor het ontwikkelen van een informatiesysteem waarmee een specifiek beslissingsproces kan worden ondersteund.

(a) het plannen van ploegendiensten

Roosterplanning is een goed voorbeeld van een individueel proces van probleemoplossen. Het maken van roosters voor werknemers in bedrijven waar in ploegen wordt gewerkt wordt meestal gedaan door éen beslisser. De probleemstruktuur omvat verschillende roosterperioden, produktie-eenheden, dienstsoorten, funkties en arbeidsvoorwaarden. Het probleem komt neer op het zodanig maken van een individueel rooster voor iedere werknemer dat die roosters

1. voldoen aan de personeelsbehoefte van de produktie-eenheden,

2. de werklast evenredig over de werknemers verdelen,

3 . in overeenstemming zijn met de geldende arbeidsvoorwaarden,

4. zoveel mogelijk tegemoet komen aan individuele wensen.

Daarbij dient het surplus aan beschikbaar personeel zo beperkt mogelijk te blijven.

Op basis van observatie van roosterplanners bij verschillende bedrijven is een taakstruktuur gemaakt die de planningsaktiviteiten van een roostermaker tamelijk nauwgezet weergeeft. Uit analyse van deze taakstruktuur blijkt dat alleen al door het automatisch bijhouden van allerlei tellingen (bijvoorbeeld gewerkte ochtend-, middag- en nachtdiensten, gewerkte weekends, totaal aantal werkuren, en inconveniëntietoeslag) een aanzienlijke tijdwinst kan worden bereikt. Verder blijkt het mogelijk de taak van het evenredig verdelen van vrije dagen over werknemers in dezelfde ploeg zodanig te struktureren dat hij door een computer met redelijk resultaat kan worden uitgevoerd. Hoewel in roosters die door deze "automatische piloot" zijn gemaakt op deze manier iets meer diensten moeten worden verschoven is dit verlies dankzij de telfaciliteit klein in verhouding tot de tijd die de automatische piloot bespaart. 
(b) het bepalen van internationale transferprijzen

Transferprijs-mutaties vormen een belangrijk financieringsinstrument voor een multinationale onderneming. Het bepalen van transferprijzen kan daarom niet los worden gezien van andere financieringsinstrumenten, zoals bijvoorbeeld dividend, royalty betalingen, en doorbelasting van kosten. De vaak ingewikkelde regelgeving van de kant van de verschillende overheden, de fiscus, de douane, en volksgezondheidsautoriteiten verhogen de complexiteit van de probleemstruktuur. Bij het vaststellen van transferprijzen zijn meerdere beslissers betrokken: fiscale specialisten, financieringsspecialisten, de prijscoördinator, en tenslotte lokaal en centraal management. Het is de taak van deze groep om wanneer zich een liquiditeitsprobleem voordoet vast te stellen welke bedragen getransfereerd moeten worden, en welke instrumenten daarvoor het meest geschikt zijn. Indien een transferprijs-mutatie wordt overwogen moeten de produkten die het meest voor een prijsverandering in aanmerking komen worden geselekteerd. Deze beslissingen worden tijdens een groepssessie genomen, waarbij de deelnemers als een team samenwerken. In feite is er sprake van één probleem dat door meerdere beslissers wordt opgelost.

Net als bij het roosterplanningsprobleem is op basis van observatie een taakstruktuur gemaakt die het besluitvormingsproces van de groep goed weergeeft. Uit deze taakstruktuur komen een aantal tekortkomingen naar voren, met als voornaamste het feit dat in principe de eerste oplossing waarmee alle betrokkenen accoord gaan wordt gekozen. Er is geen sprake van systematische generatie en evaluatie van alternatieven. In de nieuwe taakstruktuur wordt deze systematiek expliciet gemaakt, en kunnen ook andere tekortkomingen worden gecorrigeerd. Doordat hierdoor extra bladtaken worden geïdentificeerd en als beslissingsstruktuur worden weergegeven ontstaat een veel gedetailleerder beeld van de informatiebehoefte en de mogelijkheden voor computerondersteuning.

(c) het spelen van een management game

$\mathrm{Bij}$ een management game waarin een groep spelers de leiding krijgt over een produktie-onderneming is er sprake van zowel meerdere problemen (namelijk marketing, produktie, inkoop, personeel, financiën) als meerdere beslissers (namelijk de spelers). De problemen kunnen uiteraard niet geheel los van elkaar gezien worden. Dit brengt een aanzienlijk coördinatieprobleem met zich mee.

De analyse van deze taken berust ditmaal niet op observatie van beslissers. Doelstelling was om op basis van de probleembeschrijving zoals die wordt gegeven in de spelershandleiding te komen tot een speelstrategie. Het blijkt goed mogelijk om deelproblemen apart als bladtaak te analyseren, voor elk van de geïdentificeerde beslissingen het potentieel van computerondersteuning te bepalen, in de beslissingsstrukturen coördinatieproblemen aan te wijzen, en tenslotte oplossingen voor deze problemen in éen taakstruktuur te integreren. Deze taakstruktuur blijkt eenvoudig te kunnen worden aangepast wanneer zich in de probleemsituatie ingrijpende veranderingen (bijvoorbeeld een staking of een plotseling tekort aan grondstoffen) voordoen.

\section{CONCLUSIES}

Het is mogelijk gebleken een aantal concepten uit de theorie rond het oplossen van problemen te combineren tot een techniek voor het zodanig formuleren en struktureren van probleemsituaties dat een snelle vertaalslag naar informatiesystemen voor het ondersteunen van beslissingsprocessen mogelijk is. De computerondersteuning die noodzakelijk is voor het toepassen van deze techniek blijkt te kunnen worden gerealiseerd met behulp van bestaande informatietechnologie. De haalbaarheid van een objekt-georiënteerde "omgeving" voor het ondersteunen van het oplossen van problemen is hiermee aangetoond.

De bruikbaarheid van genoemde techniek blijkt uit het succes waarmee zij is toegepast op probleemsituaties voor zowel één als meerdere beslissers. Het construeren van een objektrepresentatie en het toepassen van taakanalyse op de oorspronkelijke werkwijze van beslissers leidt tot een goed begrip van het probleem. De stap van "kennen" naar "maken" wordt gezet met het ontwerpen van een nieuwe taakstruktuur en de implementatie van probleem-specifieke ondersteunende software. In elk van de onderzochte gevallen vormt de taakstruktuur een essentiële component van het uiteindelijke ondersteunende informatiesysteem. Doordat zowel de oude als de nieuwe situatie wordt vastgelegd ontstaat er een maatstaf voor de mate waarin verbetering is bereikt.

Taakanalyse resulteert niet in een expliciete koppeling van taken of beslissingen aan specifieke personen. Dit wordt echter niet ervaren als een tekortkoming. De aard en omvang van het coördinatieprobleem wordt bepaald door de opdeling in taken en niet zozeer door de kwestie "Wie doet wat?". Dit laatste kan worden gezien als onderdeel van de implementatie van de in de taakstruktuur aangebrachte coördinatiemechanismen.

Samenvattend kan worden gesteld dat de theorie die aan het begin is geformuleerd niet door de onderzoeksresultaten wordt weersproken. Met betrekking tot de generaliseerbaarheid van deze resultaten dient te worden opgemerkt dat de taakstrukturen die voor de drie besproken probleemsituaties zijn ontworpen slechts in beperkte mate zijn te veralgemeniseren. De conclusie dat de gehanteerde techniek op qua struktuur zeer uiteenlopende problemen toepasbaar is, is daarentegen wèl gerechtvaardigd. 


\section{CURRICULUM VITAE}

Pieter Bots werd op 4 februari 1963 te Oegstgeest geboren. In 1980 deed hij eindexamen Gymnasium- $\beta$ aan het St. Maartenscollege te Haren (Groningen). Het daarop volgende jaar verbleef hij in de Verenigde Staten, waar hij mede dankzij een beurs van het Institute for Educational Exchange kon studeren aan het Dickinson College in Carlisle, Pennsylvania. Van december 1980 tot juli 1981 werkte hij part-time als programmeur in dienst van DCXX Software Services. Teruggekeerd in Nederland begon hij met de studie Informatica aan de Rijksuniversiteit Leiden, alwaar hij in oktober 1985 het doctoraaldiploma behaalde. In de periode april 1983 - juli 1984 presenteerde hij de radiocursus "Programmeren met Pascal" van de Stichting Teleac. Als student-assistent aan de Leidse Faculteit der Wiskunde en Natuurwetenschappen gaf hij werkcolleges Inleiding Informatica, Datastructuren, Compilerconstructie en Informatiesystemen. Op 1 november 1985 trad hij als onderzoekmedewerker in dienst van NWO, met als gastinstelling de Technische Universiteit Delft. Sinds 1986 verzorgt hij daar het college "Ontwerpen van Beslissingsondersteunende Systemen en Expertsystemen". 


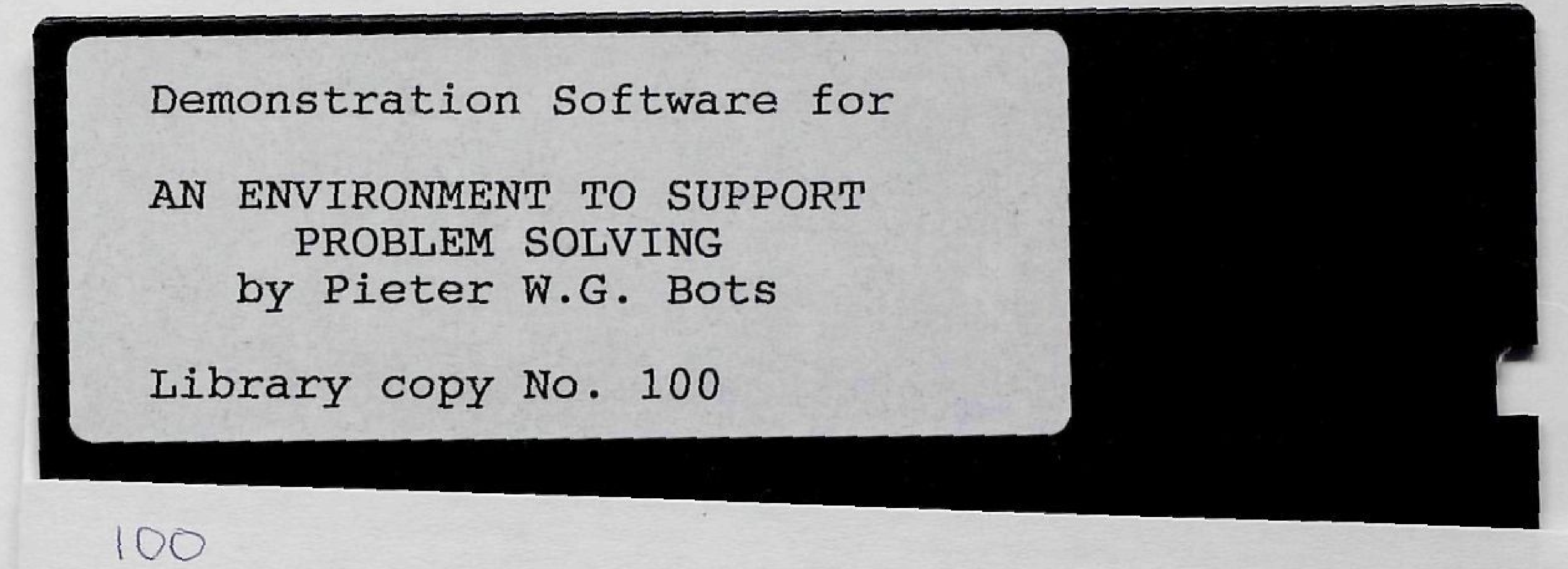

100 